\title{
Nondestructive testing of reinforced and prestressed concrete structures using acoustic waveguides
}

\author{
Komwut Wissawapaisal \\ West Virginia University
}

Follow this and additional works at: https://researchrepository.wvu.edu/etd

\section{Recommended Citation}

Wissawapaisal, Komwut, "Nondestructive testing of reinforced and prestressed concrete structures using acoustic waveguides" (2001). Graduate Theses, Dissertations, and Problem Reports. 2388.

https://researchrepository.wvu.edu/etd/2388

This Dissertation is protected by copyright and/or related rights. It has been brought to you by the The Research Repository @ WVU with permission from the rights-holder(s). You are free to use this Dissertation in any way that is permitted by the copyright and related rights legislation that applies to your use. For other uses you must obtain permission from the rights-holder(s) directly, unless additional rights are indicated by a Creative Commons license in the record and/ or on the work itself. This Dissertation has been accepted for inclusion in WVU Graduate Theses, Dissertations, and Problem Reports collection by an authorized administrator of The Research Repository @ WVU.

For more information, please contact researchrepository@mail.wvu.edu. 


\title{
NONDESTRUCTIVE TESTING OF REINFORCED AND PRESTRESSED \\ CONCRETE STRUCTURES USING ACOUSTIC WAVEGUIDES
}

Komwut Wissawapaisal

\author{
Dissertation submitted to the \\ College of Engineering and Mineral Resources \\ at West Virginia University \\ in partial fulfillment of the requirements \\ for the degree of
}

\author{
Doctor of Philosophy \\ in \\ Civil Engineering
}

\author{
Roger H.L. Chen, Ph.D., Chair \\ Hota V.S. GangaRao, Ph.D. \\ Eric K. Johnson, Ph.D. \\ Syd S. Peng, Ph.D. \\ Timothy L. Norman, Ph.D.
}

Department of Civil and Environmental Engineering

Morgantown, West Virginia

2001

Keywords: Nondestructive Testing, Reinforced Concrete, Prestressed Concrete, Acoustic Waveguides, Wave Propagation. 


\section{ABSTRACT \\ NONDESTRUCTIVE TESTING OF REINFORCED AND PRESTRESSED CONCRETE STRUCTURES USING ACOUSTIC WAVEGUIDES}

\section{Komwut Wissawapaisal}

The main objective of this research is to develop both passive and active nondestructive testing techniques for reinforced and prestressed concrete structures using acoustic waveguides. The first technique introduces a two-dimensional surface waveguide to enlarge the acoustic emission (AE) monitoring area of a reinforced concrete structure. A two-dimensional steel wire system is developed as an acoustic waveguide to study the $\mathrm{AE}$ behavior of a reinforced concrete floor slab. AE sensors are mounted to the end of the waveguides to detect $\mathrm{AE}$ signals, which are generated by pencil-lead breaks at various locations on concrete surface. Results show that the use of the surface waveguides can significantly enlarge the $\mathrm{AE}$ monitoring area. In order to identify locations of $\mathrm{AE}$ sources, a neural network system is employed. Four data sets of AE parameters and their corresponding locations are used to train the neural network system. Satisfactory results in predicting the AE source locations are obtained when using the trained neural network system to identify AE source locations of a testing data set.

The second technique involves the measurement of tensile forces in a prestressing strand in prestressed concrete structures using ultrasonic stress waves. The commonly used $1 / 2$-inch diameter seven-wire prestressing strands are studied. In this study, both experimental measurements and theoretical analysis are conducted. A stress wave is generated at one end of a prestressing strand and the wave is detected at the other end using an ultrasonic transducer. The stress waves due to tensile stresses of the strand up to $77 \%$ of its ultimate strength are investigated. The theoretical analysis is conducted by accounting for acoustoelasticity effect and the dispersion of waves. This analysis is used to calculate the traveling times of different frequency components of the wave propagating in the strand, which is subjected to prestress forces. The analysis provides a successful description of the behavior of a longitudinal transient wave traveling through a long, prestressed, circular strand. The Wigner-Ville Transform is used as a signal-processing tool in order to identify the arrival times of different frequency components of the detected waveforms. The analytical and experimental results correlate well, and good measurement accuracy is observed. Both experimental and analytical results indicate that the velocity (or traveling time) of each frequency component of the traveling wave can be related to tensile force level in the strand. This technique can effectively be used for measuring tensile forces in ungrouted post-tensioning strands of a prestressed concrete structure. 


\section{ACKNOWLEDGEMENTS}

I wish to thank my academic and research advisor Dr. Roger H.L. Chen for his invaluable guidance, encouragement, and support throughout many years of this research. A special appreciation is extended to Dr. Hota GangaRao, Dr. Eric Johnson, Dr. Syd Peng, and Dr. Timothy Norman, members of the advisory committee, for their constructive comments and suggestions.

I offer grateful thanks to my colleagues: Mr. Jeong-Hoon Choi, and Mr. Alejandro Kiriakidis for their friendship and support. Finally, I wish to express my deep gratitude to my parents for their unconditional love and understanding. 


\section{TABLE OF CONTENTS}

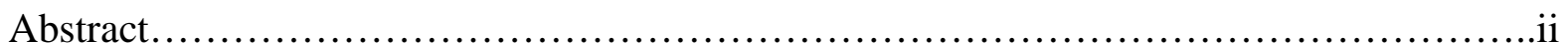

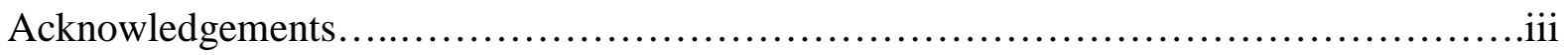

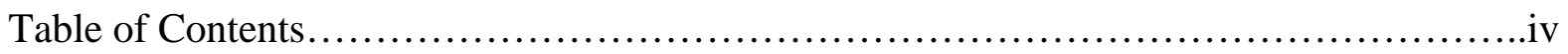

List of Tables.............................................................................

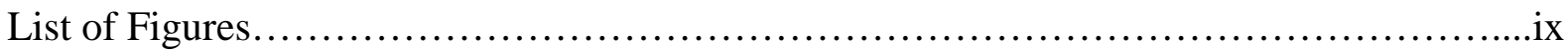

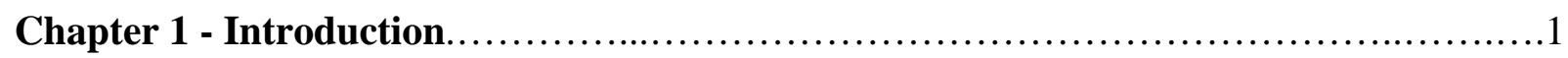

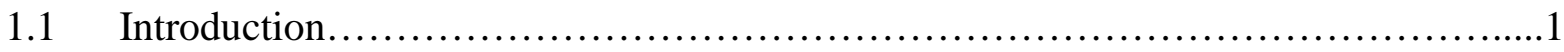

1.2 Acoustic Emission Monitoring of Reinforced Concrete Structures Using Twodimensional Surface Waveguides............................................

1.3 Measurement of Tensile Forces in Seven-wire Prestressing Strands..................6

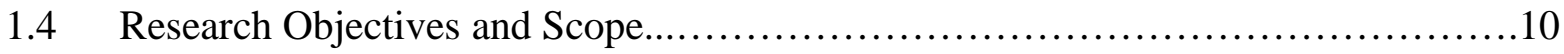

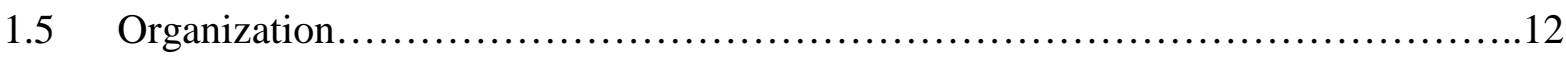

Chapter 2 - Literature Review...................................................... 14

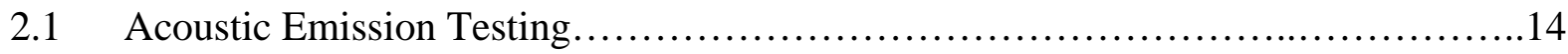

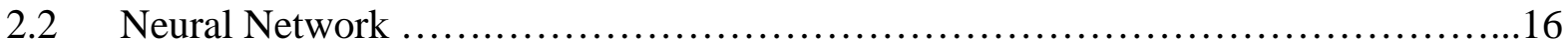

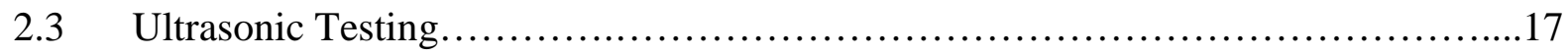

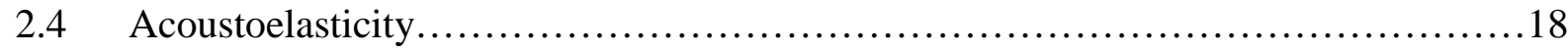




\section{Chapter 3 - Acoustic Emission Monitoring of Reinforced Concrete Structures Using}

Two-dimensional Surface Waveguides ................................20

3.1 Overview of the Acoustic Waveguide System..................................20

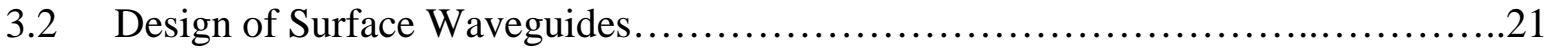

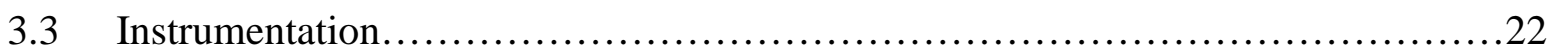

3.4 Transmission Efficiencies of AE Signal at Epoxy Joints.............................24

3.5 Frequency Components of AE Signals......................................27

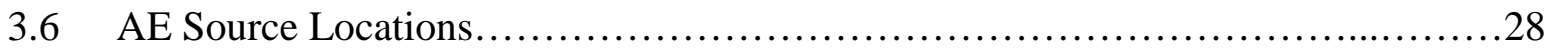

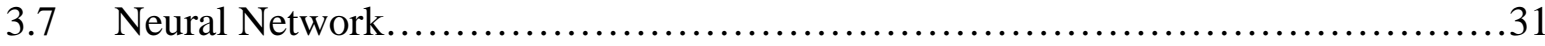

3.7.1 AE Source Locations on the Reinforced Concrete Floor Slab................31

3.7.2 Network Architecture................................................

3.7.3 Training and Testing of NN..............................................

3.7.4 NN Results and Discussions.........................................34

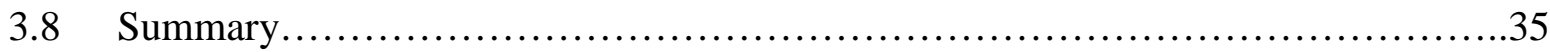

Chapter 4 - Measurement of Tensile Forces in Seven-Wire Prestressing Strands Using

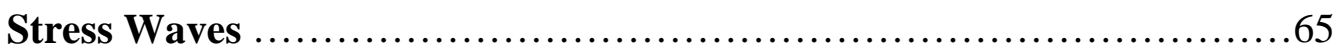

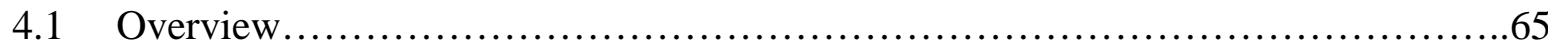

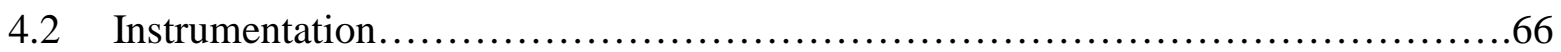

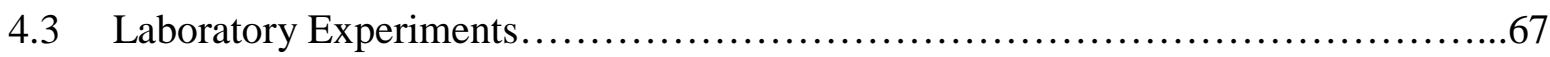

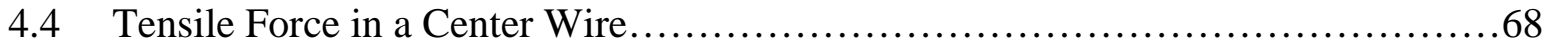

4.5 Change of Stress Wave Velocities Due to Uniaxial Stress...........................69

4.6 Wave Propagation in a Circular Waveguide.......................................70 
4.7 Numerical Analysis and Prediction.......................................72

$4.8 \quad$ Signal Observation............................................................

4.9 Peak Identification....................................................... 76

4.10 Time Shift of Waveforms due to Different Tensile Forces.......................78

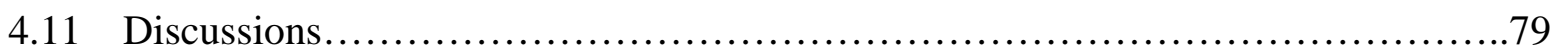

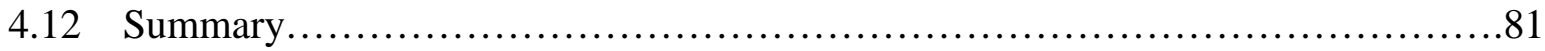

Chapter 5 - Application of Wigner-Ville Transform to Evaluate Tensile Forces in SevenWire Prestressing Strands .......................................... 99

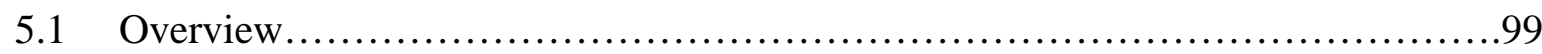

5.2 Wigner-Ville Transform................................................. 100

$5.3 \quad$ Laboratory Experiments.............................................. 102

5.4 Experimental Results and Discussions................................... 103

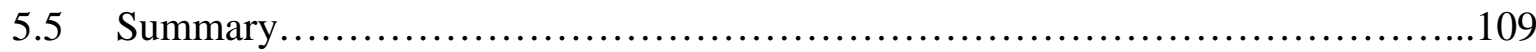

Chapter 6 - Field Measurements of Tensile Forces in Seven-wire Prestressing

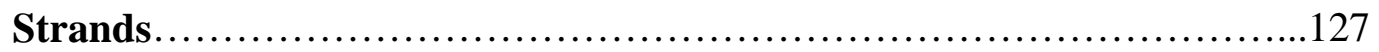

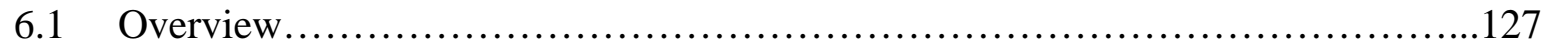

6.2 Post-Tensioning Strands............................................... 128

6.2.1 Marietta Experiment........................................... 128

6.2.2 Winchester Experiment......................................... 133

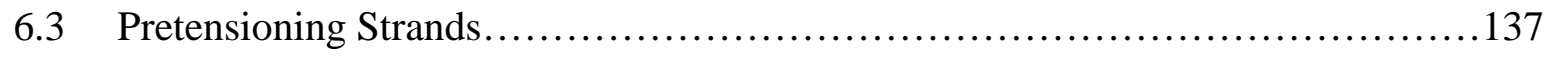

6.3.1 Marietta Experiment............................................. 137 


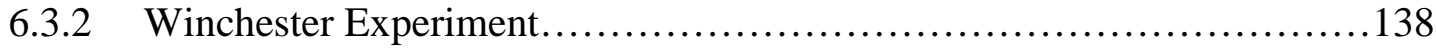

6.3.3 Attenuation of Stress Waves in the Seven-Wire Strands

Bonded with Concrete............................................139

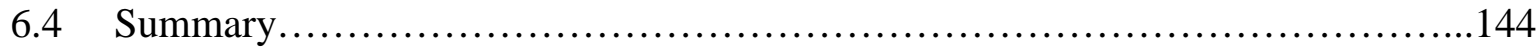

Chapter 7 - Conclusions and Recommendations .................................. 181

7.1 Acoustic Emission Monitoring of Reinforced Concrete Structures Using Twodimensional Surface Waveguides.......................................181

7.2 Tensile Force Measurement of Seven-wire Prestressing Strands Using

Stress Waves......................................................... 183

References................................................................ 190

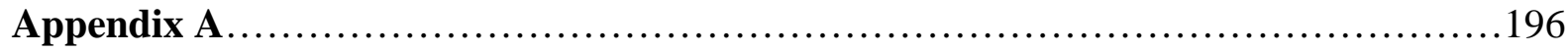

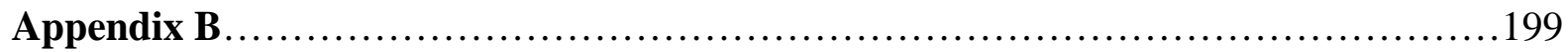

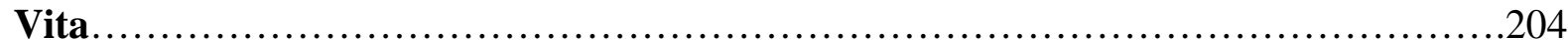




\section{LIST OF TABLES}

Table 3.1 Comparison between actual and predicted pencil-lead break locations

Table 4.1 Calculated material constants of the seven-wire prestressing strand

(Grade 250)

Table 4.2 Change of group velocity due to tensile stresses in the center wire $(\varnothing=4.2 \mathrm{~mm})$

Table 4.3 Calculated time shift of different frequency components

Table 5.1 Calculated material constants of the seven-wire prestressing strand

(Grade 250)

Table 5.2 Variations of group velocity and tensile force due to $\pm 1 \%$ change of the strand's material constants. (evaluated at propagating frequency $=$ $300 \mathrm{kHz}$, tensile force $=28 \mathrm{kips}, \mathrm{C}_{\mathrm{g}}=4957 \mathrm{~m} / \mathrm{s}$ ). The $1 / 2-i n c h$ seven-wire strand Grade 250 is used. ..

Table 5.3 Calculated material constants of the seven-wire prestressing strand

(Grade 270).

Table 6.1 Calculated material constants of a seven-wire prestressing strand (Grade 270)

from the field measurement at Marietta Structures Corp.

Table 6.2 Variations of group velocity and tensile force due to change of the strand's material constants. (evaluated at propagating frequency $=350 \mathrm{kHz}$, tensile force $=32 \mathrm{kips}, \mathrm{C}_{\mathrm{g}}=4940 \mathrm{~m} / \mathrm{s}$ ). The $1 / 2$-inch seven-wire strand Grade 270 is used

Table 6.3 Calculated material constants of a seven-wire prestressing strand (Grade 270) from the field measurement at Shockey Bros., Inc

Table 6.4 Calculated group velocities and arrival times of several frequency components of the detected waveforms due to different tensile forces. 


\section{LIST OF FIGURES}

Figure $3.1 \quad$ Experimental setup and instrumentation.............................. 38

Figure $3.2 \quad$ Wave propagation at an epoxy joint................................... 39

Figure 3.3 Transmission directions of AE signals at epoxy joints..................40

Figure $3.4 \quad$ AE parameters...................................................41

Figure $3.5 \quad$ Calibration chart of the AE sensor.....................................42

Figure 3.6 Experimental setup for transmission efficiency determination of surface waveguides (Case A) .......................................43

Figure 3.7 Experimental setup for transmission efficiency determination of

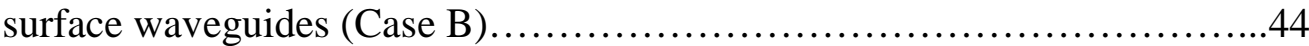

Figure 3.8 Experimental setup for transmission efficiency determination of surface waveguides (Case C)

Figure 3.9 Experimental setup for transmission efficiency determination of

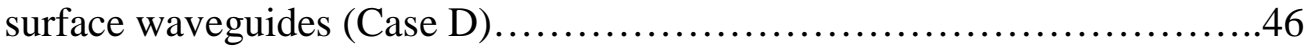

Figure 3.10 Experimental setup for transmission efficiency determination of surface waveguides (Case E) ...................................47

Figure 3.11 Experimental setup for attenuation measurement of concrete.............48

Figure 3.12 Propagation directions of AE signal in the surface waveguide..............49

Figure 3.13 Attenuation curves of AE signals propagating in concrete and in surface waveguides...............................................50

Figure 3.14 Experimental setup for the characteristic study of AE signal generated by standard pencil-lead breaks....................................51

Figure 3.15 Typical AE signal generated by a pencil-lead break.....................52

Figure 3.16 Frequency components of a typical AE signal generated by a pencil-lead break....................................................

Figure 3.17 An example of an AE signal detected at the AE sensor after propagating through several epoxy joints. 
Figure 3.18 Frequency components of the AE signal detected at the AE sensor after propagating through several epoxy joints....

Figure 3.19 Coordinate system and pencil-lead break locations on the reinforced concrete floor slab..............................................56

Figure 3.20 Detected AE signals due to a pencil-lead break at coordinate $(1.2,0.4)$ .57

Figure 3.21 Predicted propagation routes due to the pencil-lead break at

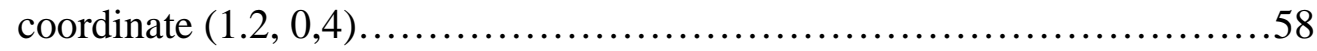

Figure 3.22 Schematic diagram of AE data processing system......................59

Figure 3.23 Architecture of the NN........................................60

Figure 3.24 Correct rates of the testing data set versus number of hidden units of the NN.

Figure 3.25 RMS value of the testing data set versus number of hidden

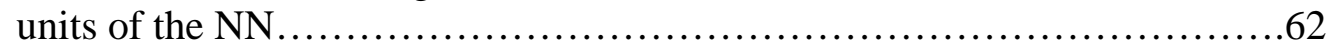

Figure 3.26 Graphical results of the NN predictions.............................63

Figure 3.27 Prediction error of pencil-lead break locations from NN.................64

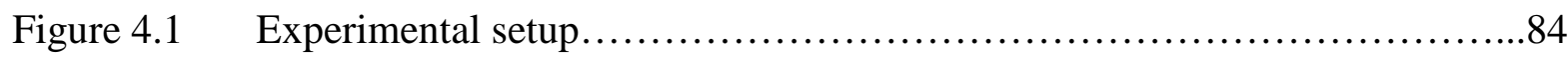

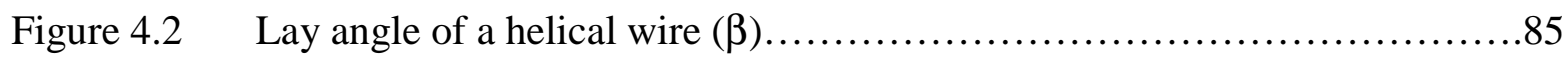

Figure 4.3 Group velocity of the first two longitudinal modes for the center wire $(\varnothing=4.2 \mathrm{~mm})$. There is no tension applied......................86

Figure 4.4 Waveform recorded at receiving end of the strand subjected to $10 \mathrm{kips}$ tensile force...........................................87

Figure $4.5 \quad$ Early part of the waveform due to 10 kips tensile force....................88

Figure 4.6 Frequency components of the first 5 and the first 18 cycles of the waveforms due to different tensile forces................................89

Figure 4.7 Arrival part of the waveform due to 0 kips tensile force..................90

Figure 4.8 Arrival part of the waveform due to 10 kips tensile force..................91

Figure 4.9 Arrival part of the waveform due to 20 kips tensile force.................92 
Figure 4.10 Time shift of waveforms due to an increase in tensile force from 10 kips to 15 kips............................................93

Figure 4.11 Time shift of the first 18 cycles of the waveform due to an

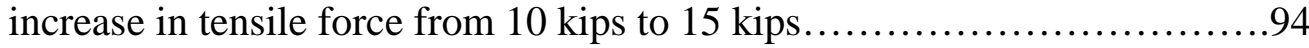

Figure 4.12 Time shift of the waveforms due to changes in tensile force.................95

Figure 4.13 Comparison of shape and amplitude between waveforms recorded at 24 kips and 25 kips tensile forces...................................96

Figure 4.14 Peak arrival times of the first five cycles of the waveforms................97

Figure 4.15 Time shift comparison between experimental and analytical results for the first 5 cycles of the waveforms............................98

Figure $5.1 \quad$ Experimental setup.................................................. 113

Figure 5.2 Recorded waveforms due to 15 kips and 20 kips tensile forces. The $1 / 2$-inch seven-wire strand Grade 250 is used..........................114

Figure 5.3 Frequency components of the recorded waveform due to 15 kips tensile force. The $1 / 2$-inch seven-wire strand Grade 250 is used....

Figure 5.4 Time-frequency relationship of the recorded waveform due to 15 kips tensile force. The $1 / 2$-inch seven-wire strand Grade 250 is used..............116

Figure 5.5 Time-frequency relationship of the recorded waveform due to 20 kips tensile force. The $1 / 2$-inch seven-wire strand Grade 250 is used....

Figure 5.6 Time-frequency relationship of the recorded waveform due to $25 \mathrm{kips}$ tensile force. The $1 / 2$-inch seven-wire strand Grade 250 is used.............118

Figure 5.7 Group velocity of the first two longitudinal modes for the center wire $(\varnothing=4.2 \mathrm{~mm})$. There is no tensile force applied.

Figure 5.8 Time-frequency relationships of waveforms due to 15 kips, 20 kips, and 25 kips tensile forces.

Figure 5.9 Time-frequency relationship of the recorded waveform due to 12 kips tensile force. The 1/2-inch seven-wire strand Grade 270 is used.

Figure 5.10 Time-frequency relationship of the recorded waveform due to $16 \mathrm{kips}$ tensile force. The $1 / 2$-inch seven-wire strand Grade 270 is used. 
Figure 5.11 Time-frequency relationship of the recorded waveform due to $20 \mathrm{kips}$ tensile force. The $1 / 2$-inch seven-wire strand Grade 270 is used............123

Figure 5.12 Group velocity of the first two longitudinal modes for the center wire $(\varnothing=4.4 \mathrm{~mm})$. There is no tensile force applied.

Figure 5.13 Time-frequency relationships of waveforms due to $12 \mathrm{kips,}$ 16 kips, and 20 kips tensile forces....

Figure 5.14 (a) Comparison between time-frequency relationships of two different strands subjected to 28 kips tensile force

(b) Time shift comparison between waveforms detected from these two strands

Figure $6.1 \quad$ Experimental setup...........................................149

Figure $6.2 \quad$ Pulsing end of the prestressing strand.............................. 150

Figure 6.3 Receiving and loading end of the prestressing strand...................151

Figure 6.4 Connection between the output transducer and the seven-wire prestressing strand.

Figure 6.5 Arriving waveform recorded at the receiving end of the strand subjected to 20 kips tensile force.

Figure 6.6 Frequency components of the recorded waveform due to 20 kips tensile force.

Figure 6.7 Time-frequency relationship of the recorded waveform due to 20 kips tensile force.

Figure 6.8 Time-frequency relationship of the recorded waveform due to 26 kips tensile force.

Figure 6.9 Time-frequency relationship of the recorded waveform due to $32 \mathrm{kips}$ tensile force....................................................... 157

Figure 6.10 Group velocity of the first two longitudinal modes for the center wire $(\varnothing=4.4 \mathrm{~mm})$. There is no tensile force applied. 158

Figure 6.11 Time-frequency relationships of waveforms due to 20 kips, 26 kips, and 32 kips tensile forces

Figure 6.12 Arrival time of $340 \mathrm{kHz}, 350 \mathrm{kHz}$, and $360 \mathrm{kHz}$ frequency components due to increasing tensile forces. 
Figure 6.13 Reproducibility of two recorded waveforms due to 32 kips

tensile force.

Figure $6.14 \quad$ Inverted Tee Girder.............................................. 162

Figure 6.15 Loading end of the prestressing strands............................ 163

Figure 6.16 Monostrand system............................................... 164

Figure 6.17 Experimental setup using pulse generator............................165

Figure 6.18 Experimental setup at the receiving end of the strand.....................166

Figure 6.19 Experimental setup using impact hammer.......................... 167

Figure $6.20 \quad$ Impact excitation....................................................

Figure 6.21 Arriving stress wave signal due to 10 kips tensile force...................169

Figure 6.22 Typical frequency components of detected waveforms due to 10 kips tensile force............................................... 170

Figure 6.23 WVT plot of the detected waveform due to 10 kips tensile force...........171

Figure 6.24 Time shift of the waveform due to changes in tensile force................172

Figure 6.25 Arrival times of the waveforms due to different tensile forces.............173

Figure 6.26 Group velocity of the first two longitudinal modes for the center wire $(\varnothing=4.4 \mathrm{~mm})$. There is no tensile force applied...................174.

Figure 6.27 Pretensioned concrete box girder................................. 175

Figure 6.28 Experimental setup for pretensioned concrete box girder................176

Figure 6.29 Peak amplitudes of recorded stress waves due to different strand length....177

Figure 6.30 Experimental setup for determining the attenuation of the stress wave due to bonding between the seven-wire strand and concrete...............178

Figure 6.31 Recorded stress waves due to different bonding length between the seven-wire strand and concrete.

Figure 6.32 Attenuation of peak amplitudes of the stress waves due to bonding between the strand and concrete. 
Figure 7.1 Relative change in group velocity due to tensile stress in the $1 / 2$-inch seven-

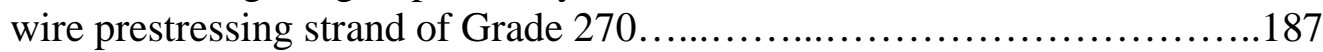

Figure 7.2 Relationship between $K(\omega)$ and propagation frequency of a stress wave....................................................... 188

Figure 7.3 Dispersion curves due to 0 kips and 30 kips tensile force in

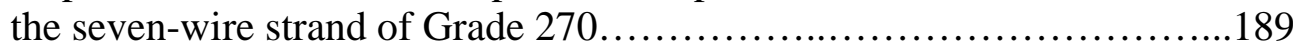

Figure B-1 Coordinate system of a circular waveguide ...........................203 


\section{CHAPTER 1}

\section{INTRODUCTION}

\subsection{INTRODUCTION}

Nondestructive testing (NDT) is a descriptive term used for the examination of materials and components without changing or destroying their usefulness. NDT plays a crucial role necessary to assure safety and reliability. Typical examples are found in aircraft, motor vehicles, pipelines, bridges, trains, power stations, refineries and oil platforms which can all be inspected using NDT. NDT is also a quality assurance management tool. It can give impressive results when used correctly. It requires an understanding of the various methods available and their capabilities and limitations. Materials, products, and equipment which fail to achieve their design requirements or projected life due to undetected defects may require expensive repair or early replacement. Such defects may also be the cause of unsafe conditions or catastrophic failure. Materials can be examined using NDT and either accepted, rejected or repaired. NDT techniques can also be used to monitor the integrity of a structure throughout its service life. The method that can be used for NDT depends on the physical properties of the materials.

In this dissertation, two NDT techniques are developed for damage inspection of concrete structures. These techniques include (1) acoustic emission monitoring of reinforced concrete structures using two-dimensional surface waveguides, and (2) ultrasonic 
method for measurement of tensile forces in seven-wire prestressing strands for prestressed concrete structures.

\subsection{ACOUSTIC EMISSION MONITORING OF REINFORCED CONCRETE STRUCTURES USING TWO-DIMENSIONAL SURFACE WAVEGUIDES}

The first NDT technique involves the acoustic emission (AE) monitoring of reinforced concrete structures, which is a passive way of damage monitoring. AE refers to the elastic energy that is spontaneously released by materials when they undergo deformation. AE signals can take many forms depending on the material and the failure mechanism in the material. Two basic types of AE signals can be generated, which are continuous emission and burst emission. The continuous emission consists of a large number of low amplitude pulses. Continuous emission is a qualitative description of the sustained signal level produced by rapidly occuring acoustic emission events. These are generated by events such as plastic deformations in metals (Bray and McBride, 1992). Burst type emission signals, on the other hand, are high amplitude signals with short durations. Burst emission is a qualitative description of the discrete signal related to an individual emisson event occuring within the material. The emission of this type may occur during crack growth or fracture in brittle materials. Acoustic emission in concrete structure is due primarily to cracking processes, slippage between concrete and steel reinforcement, and fracture or debonding of reinforcement. AE signals in concrete are categorized in the burst emission type. 
AE monitoring is one of the NDT techniques that is capable for monitoring structural integrity, for detecting leaks and incipient failures in mechanical equipment, and for characterizing materials behavior. AE monitoring technique has many advantages over other nondestructive testing techniques because of its capability to detect active damages of existing structures. It has been used with many different materials such as metal, concrete, plastic, glass fiber, and many types of laminated and fiber-reinforced materials. The use of this technique can be seen in monitoring of many types of components from large oil storage tanks to small electronic components (Boving 1989). AE monitoring can be conducted in various aspects such as:

- Production monitoring, where a component is stressed according to a specified testing procedure during AE monitoring. The loading is scheduled to achieve the optimum AE activity, e.g. by stepwise stressing, so that AE can be measured both during the increasing stress period and during the constant stress period.

- Condition monitoring, where components are subjected to a loading procedure, as described above, after a predetermined period of operation. In such cases, it is often useful to be able to compare with previous AE measurements so that the development of any defects can be traced.

- Service Monitoring, where AE equipment is permanently mounted on the component in order to provide an alarm in case that critical AE signals are detected. 
In the above mentioned types of monitoring, the size and shape of components are critical to the choice of $\mathrm{AE}$ instrumentation. Because $\mathrm{AE}$ signals generated from a defect are attenuated during propagation in the material, it is necessary to determine the maximum permissible distance between $\mathrm{AE}$ sensors required to achieve certain detection sensitivity. Appropriate AE equipment has been developed in recent years for each type of applications. The equipment may have different capabilities for analyzing the signals arriving at the $\mathrm{AE}$ sensors. The following types are normally used for AE analyses:

- analysis of signal amplitude as a function of stress and time

- analysis of the number of signals which exceed a reference threshold

- computation of the energy of the detected signals

- analysis of the frequency content of the signals

- localization of the signal source

- correlation analysis, where each detected signal is compared with previously recorded signals

Some AE equipment can perform combinations of the above mentioned analysis tasks. For example, detection of signal amplitude is often carried out in connection with localization, so both the magnitude and the position of the AE source can be obtained.

The major difficulty in AE monitoring of concrete structures is the high signal attenuation in concrete. Therefore, the AE monitoring of concrete structures is very limited without providing a large number of $\mathrm{AE}$ sensors and associated data acquisition systems. One way to overcome this difficulty is to use acoustic waveguides. In general, acoustic 
waveguides are either mounted on or embedded in the main structures so that they can be used to convey AE signals from their sources to the monitoring devices. An efficient waveguide material, such as steel or aluminum, would be able to provide a better signal transmission than concrete. By using waveguides, AE signals are able to propagate a longer distance in the waveguides so that a larger area of concrete can be monitored without sacrificing a large number of monitoring devices. The use of waveguides can also be extended to the AE monitoring of other highly attenuative materials such as fiber-reinforced plastic and soil (e.g. soil slope stability, ground movement). Waveguides are very useful when the monitoring site is inaccessible or when it is not possible to install AE sensors (e.g. extremely high temperature and radioactive environments). In this study, a two-dimensional (2-D) acoustic surface waveguide system is developed.

Due to the inhomogeneous nature of concrete and the complexity of the propagating AE signals in the 2-D acoustic surface waveguide system, each detected AE signal has its own characteristics that can not easily be predicted. This creates difficulties in identifying the AE source locations. For this reason, a Neural Network (NN) is used to locate the AE sources. $\mathrm{NN}$ is known for its ability of mapping problems that are tolerant of a high error rate as well as mapping problems that have a lot of example data available but to which mathematical relationships cannot be easily derived. The application of NN for predicting locations of the AE sources is described in a later chapter. 


\subsection{MEASUREMENT OF TENSILE FORCES IN SEVEN-WIRE PRESTRESSING STRANDS}

The second NDT technique is an active monitoring technique for the measurement of tensile forces in seven-wire prestressing strands for prestressed concrete structures. The need of this monitoring technique is due to the fact that initial prestressing forces applied to these prestressing strands undergo a progressive process of reduction over many years of their service lives. The reduction in the prestressing forces can be categorized into two main groups: (1) immediate losses during the fabrication or construction process, including elastic shortening of the concrete, anchorage losses, and frictional losses, and (2) time-dependent losses such as creep, shrinkage, and those due to temperature effects and steel relaxation. However, unexpected losses of prestressing forces may occur due to direct damage of concrete structures and corrosion of prestressing strands. In many cases, losses of tensile forces in prestressing strands have caused catastrophic failures of concrete structures. For this reason, it is important to periodically measure the prestressing force in the strands so that structural integrity of the existing concrete members can be evaluated.

In this study, the ultrasonic stress wave technique is employed. Ultrasonic nondestructive testing is a versatile technique that can be applied to a wide variety of material analysis applications. While ultrasonic NDT is better known in its more common applications for thickness gauging, flaw detection, and acoustic imaging, high frequency acoustic waves can also be used to discriminate and quantify some basic mechanical, structural, or compositional properties of solids and liquids. Ultrasonic technique is based on a simple principle of physics: the motion of any wave will be affected by the medium 
through which it travels. Thus, changes in one or more of parameters associated with the passage of a high frequency stress wave through a material (e.g. traveling time, attenuation, scattering, and frequency content) can be correlated with changes in physical properties such as hardness, elastic modulus, density, homogeneity, or grain structure.

Ultrasonic technique utilizes the range of frequencies from approximately $20 \mathrm{kHz}$ to over $100 \mathrm{MHz}$. Both longitudinal and shear (transverse) modes of vibration are commonly employed, as well as surface (Rayleigh) waves and plate (Lamb) waves in some specialized cases. Because shorter wavelengths are more responsive to changes in the medium through which they pass, many material analysis applications will benefit from using the highest frequency that the test specimen will support. Stress wave pulses are normally generated and received by piezoelectric transducers that have been coupled to the test material. In most cases a single transducer, coupled to one side of the test specimen, serves as both transmitter and receiver (pulse/echo mode), although in some situations, involving highly attenuating or scattering materials, separate transmitting and receiving transducers on opposite sides of the part are used (through transmission mode). A stress wave is launched by exciting the transducer with either a voltage spike or a continuous wave impulse. The wave travels through the test material, either reflecting off the far side to return to its point of origin (pulse/echo), or being received by another transducer at that point (through transmission). The received signal is then amplified and analyzed. A variety of commercial instrumentation is available for this purpose, utilizing both analog and digital signal processing.

The relevant measurement parameters will typically be one or more of the following: 
- Wave velocity/pulse traveling time: Wave velocity is the simpliest ultrasonic parameter to measure. The speed of waves in a homogenous medium is directly related to both elastic modulus and density. Thus, changes in either elasticity or density will affect pulse traveling time through a sample of a given thickness. Additionally, varying degrees of nonhomogeneity and stress inside the medium (acoustoelasticity) may have an effect on wave velocity.

- Attenuation: Wave energy is absorbed or attenuated at different rates in different materials, governed in a complex fashion by interactive effects of density, hardness, viscosity, and molecular structure. Attenuation normally increases with frequency in a given material.

- Scattering: Waves reflect from boundaries between dissimilar materials. Changes in grain structure, fiber orientation, porosity, particle concentration, and other microstructural variations can affect the amplitude, direction, and frequency content of scattered signals. Scatter effects can also be monitored indirectly by looking at changes in the amplitude of a pulse echo or a through-transmission signal.

- Frequency (Spectrum) content: All materials tend to act to some degree as a low pass filter, attenuating or scattering the higher frequency components of a broad band stress wave more than the lower frequency components. Thus, analysis of changes in the remaining frequency content of a selected broadband pulse that has passed through the test material can track the combined effects of attenuation and scattering as described above.

In some applications ultrasonic data such as velocity can be directly used to calculate properties such as elastic modulus. In other cases, ultrasonic testing is a comparative 
technique so that in order to establish a test protocol in a given application, it will be necessary to experimentally evaluate reference standards representing the range of material conditions being quantified. From such standards it will be possible to record how wave transmission parameters vary with changes in specific material properties, and then from this baseline information, it will be possible to identify or predict similar changes in test samples.

The underlying concepts of this research are related to (1) acoustoelastic effect, which refers to the changes in the velocity of elastic waves propagating in a body which is simultaneous undergoing static elastic deformation, and (2) dispersive behavior of elastic waves propagating in a medium, which implies that different frequency components of the stress wave propagate with different velocities. In other words, we would be able to measure the tensile force level in a prestressing strand if we could measure the traveling time of one particular frequency component of a stress wave that is propagating through the entire length of the strand. 


\subsection{RESEARCH OBJECTIVES AND SCOPE}

There are two major objectives in this dissertation:

\section{A) Development of Two-dimensional Surface Waveguides}

\section{$\underline{\text { Objectives }}$}

The main objectives of this study are: (1) to enlarge the AE monitoring area of concrete structures; and (2) to identify locations of the AE sources. The proposed waveguide system will be able to provide efficient detection of AE activities in concrete structures so as to overcome the difficulty of high attenuation in concrete.

\section{$\underline{\text { Scope }}$}

The scope of this study includes the design and construction of a two-dimensional surface waveguide system capable of detecting $\mathrm{AE}$ activities for large area of concrete structures. Three types of acoustic waveguide joints are developed and are evaluated for their signal transmission efficiencies. A reinforced concrete floor slab is used as a test structure. Standard pencil-lead breaks are performed on the reinforced floor slab in order to produce $\mathrm{AE}$ signals. In order to identify the $\mathrm{AE}$ source locations, an artificial neural network is used to predict the locations of the generated AE signals on the concrete floor. Finally, prediction results of the AE source locations are investigated. 


\section{B) Tensile Force Measurement of the Seven-wire Prestressing Strands}

\section{$\underline{\text { Objectives }}$}

The main objectives of this study are: (1) to develop a nondestructive evaluation technique for determining tensile forces in the seven-wire prestressing strands for prestressed concrete structures; (2) to investigate the behavior of a stress wave propagating in the prestressing strand subjected to tensile stresses; (3) to develop an analytical tool for predicting the stress wave behavior in the prestressing strands subjected to tensile forces; and (4) to apply an appropriate signal processing technique for analyzing stress wave motions simultaneously in time and frequency domains.

\section{$\underline{\text { Scope }}$}

The scope of this study includes the experimental and analytical studies of tensile force prediction of the $1 / 2$-inch diameter seven-wire steel strands, which are commonly used as prestressing strands in construction. The ultrasonic method is used where the velocity (or traveling time) of a stress wave propagating through the entire length of each strand is measured. Different tensile forces within the strand's linear elastic range are applied. In order to simplify both experiments and analyses, only the longitudinal wave motions inside the center wire of the strand are monitored. In this study, the strand is considered to be a slender, isotropic, elastic circular waveguide. Theoretical study is conducted based on the frequency equation of a waveguide with circular cross-section. The acoustoelastic effect of an elastic solid is also taken into consideration. The relationship between the wave speed 
and the tensile force is established. The Wigner-Ville transform is employed in order to analyzed the recorded stress waves. Prestressing strands of different lengths and grades are tested at a laboratory and at prestressed concrete manufacturers where both pretensioned and post-tensioned strands inside actual prestressed concrete structures are tested. Accuracy of the experimental measurements and analyses is investigated. Finally, analytical and experimental precedures for the strands' tensile force measurement are established.

\subsection{ORGANIZATION}

The content of this dissertation includes 7 chapters. Chapter 1 presents the introduction of this research and its use in nondestructive detection of concrete structures. The underlying concepts of the proposed nondestructive evaluation method using acoustic emission and ultrasonic methods are presented. In addition, this chapter also presents the objectives and scope of this research. Chapter 2 presents a comprehensive literature review which discusses several previous studies on acoustic emission and ultrasonic inspection methods for various types of structural members. Chapter 3 describes the development of the two-dimensional surface waveguide for acoustic emission monitoring of reinforced concrete structures. The use of the neural network for locating acoustic emission sources on a concrete floor slab is illustrated in this chapter. Chapter 4 presents the fundamental theory and assumptions of the wave propagation in the seven-wire prestressing strand together with the development of the experimental technique. Experimental study of the propagating stress wave subjected to the change in the strand's tensile stress is also described in this chapter. Chapter 5 demonstrates the use of an appropriate signal processing technique in 
order to establish the relationship between the velocity of the traveling wave and the tensile stress level in the strand. Chapter 6 describes the field measurements of tensile forces in the prestressing strands at two concrete manufacturers where the ability of this technique to measure tensile forces in both pretensioned and post-tensioned concrete structures is demonstrated. Chapter 7 presents conclusions and recommendations.

It is noted that both NDT techniques developed in this study can be cooperatively applied on a given concrete structure. For instance, application of two-dimensional surface waveguides on a prestressed concrete girder would be able to indicate whether there are any active cracks. An unexpected crack may be due to the loss of tensile forces in the prestressing strands. By using stress wave technique, the tensile forces in these strands can be measured so that the extent of the damage to the girder can be evaluated. 


\section{CHAPTER 2}

\section{LITERATURE REVIEW}

\subsection{ACOUSTIC EMISSION TESTING}

The study of AE can be traced back to the 1930s when Friedrich Forster and Erich Scheil studied the noise caused by the formation of nickel-steel (Miller and McIntyre, 1987). The first experiments with AE measurements of materials such as metals and wood were carried out in the early 1950 s by Joseph Kaiser. Using sensitive electronic instruments he could detect sounds from many different metals subjected to deformation. He also discovered that a material which had been previously stressed exhibited AE again when the previous stress was exceeded. The AE phenomenon of this type was then called "Kaiser Effect". Following Kaiser's work, Shofield, Tatro, and Malvern had applied AE techniques into various research fields of material engineering. The industrial application of AE began in the 1960s. Pioneers such as Green, Lockman, Dunegan, and Hartbower, started to apply AE techniques on aerospace and nuclear industries. Since the mid 1980's, AE techniques have been used extensively due to the advancement of computer technology and the availability of other sophisticated equipment. AE techniques have been applied in the petroleum and chemical industries for leakage detection and structural inspection of pressure vessels, pipelines, and storage tanks. In the electronic industry, AE techniques have been used for bonding tests of intergrated circuits. In the aerospace industry, AE techniques have 
been used for component and structural testings. AE techniques have been used extensively in material engineering. In civil engineering, $\mathrm{AE}$ techniques have been applied in material studies (such as soil, rock, steel, and concrete), in welding inspection, and in structural monitoring. Gong et.al. (1992) reported AE monitoring of steel railroad bridges. Woodward (1983) reported an AE monitoring on anchorages of a prestressing bridge. Other works on AE monitoring of steel structures can also be found from Hutton and Skorpik (1978), Hopwood (1976), and Dunegan (1985).

Several researchers have used the AE techniques for monitoring cracks in concrete (Chen et.al., 1992; Hearn and Shield, 1997; Kimura. et. al. 1988; Lim and Koo, 1989; Lovass, 1985; Maji and Shah, 1988; Maliszkiewicz, 1990; Matsuyama et.al, 1993; Ohtsu ,1986,1987, 1988, 1998; Olivito and Surace, 1997; Ouyang et. al., 1991; Umoto, 1987; and Yuyama et.al., 1988, 1994, 1995a, 1995b) and for monitoring of debonding and corrosion of steel reinforcement in concrete (Hawkins et.al., 1988; Li et.al., 1998; and Yoon et.al., 2000). Due to the fact that concrete is a highly attenuative material, it requires a large number of sensors to monitor an entire reinforced concrete structure. Similar observations were reported for structures made of FRP materials (Pollock, 1989). For this reason, acoustic waveguides were introduced. Since 1980, acoustic waveguides have been used to detect the AE signals of buried pipelines (Dunegan Testing Group, 1989). An investigation of embedded acoustic waveguides for damage monitoring of reinforced concrete structures was reported by Chen and He (1996). The study of acoustic surface waveguides was first reported by Chen and $\mathrm{He}$ (1995). In this literature, a single steel wire mounted on the surface of a concrete beam was used as a waveguide. It was called "one-dimensional surface waveguide". A relatively high transmission efficiency of the waveguide was obtained. 
However, the ability to detect the AE source locations only in the longitudinal direction (1 dimension) along its length is a limitation of the one-dimensional surface waveguide. In order to monitor the AE activities of a large concrete surface area (2 dimensions), the socalled "two-dimensional acoustic surface waveguide system" is developed in this study.

\subsection{NEURAL NETWORK}

A neural network (NN) is an information-processing paradigm inspired by the way the densely interconnected, parallel structure of the human brain processes information. NN is a collection of mathematical models that emulate some of the observed properties of biological nervous systems and draw on the analogies of adaptive biological learning. The key element of the NN paradigm is the novel structure of the information processing system. The advantage of NN lies in its resilience against distortions in the input data and its capability of learning. $\mathrm{NN}$ is good at solving problems that are too complex for calculations (e.g., problems that do not have an algorithmic solution or for which an algorithmic solution is too complex to be found).

NN has been successfully applied to several applications, such as text to speech synthesis (Sejnoeski and Rosenberg, 1986), handwritten recognition (Cun et. al., 1989; Cun and Touretzky, 1990; Denker et.al., 1989), speech recognition (Waibel et.al., 1989; Yuhas et.al, 1989) and signal classification (Gorman and Sejnowski, 1988; Cun, 1989; Prabhu et.al., 1991). Applications of NN in AE signal processing were reported by Grabec and Sachse (1989), and by Chen and Chen (1992). Their results demonstrated the capability of NN in solving AE source inversion problems. In this study, the use of $\mathrm{NN}$ in processing the 
traditional AE parameters is presented. These AE parameters are transformed into a compatible format that can be applied to the NN. The architecture and learning algorithm of the NN is described. The NN system is applied to AE experimental data and results of the source location prediction are compared with the experimental observations.

\subsection{ULTRASONIC TESTING}

The first significant work in ultrasonic techniques was accomplished by Langevin of France, who experimented with ultrasonic submarine detection methods during World War I (Bray and McBride, 1992). Langevin used quartz crystals to transmit and receive ultrasonic signals by enhancing the piezoeletric effect of crystals to a usable level. In 1918, he was

able to detect echoes from a submarine at a distance of $1.5 \mathrm{~km}$. Experiments were conducted by Boyle and by Wood and Loomis (1927), using quartz piezoelectric transducers. In 1921, Behun reported a method of measuring the depth of the seabed by an ultrasonic resonance method. In 1929 and 1935, Sokolov discussed the use of ultrasonic waves in detecting defects in metal objects. In 1931, Mulhauser obtained a patent using ultrasonic waves to detect flaws in solids, using a transmission mode operating with two transducers (one to transmit the other to receive the ultrasonic waves). Sokolov carried out many pioneering experiments in ultrasonic testing such as investigating coupling liquids, matching impedance, and developing methods to obtain an image of a defect by a scanning technique. In 1940, Schraiber developed methods of continuous ultrasonic wave testing (Mullins, 1964). Ervin (1945) reported on thickness measurement by ultrasonic methods (Krautkramer and Krautkramer, 1983). 
A very important development came with the study of pulsed ultrasonic testing by Firestone (1940) and by Simmons (1945). This method was shown to be relatively fast and simple. Present day methods of ultrasonic testing evolved from this method. Firestone designed an instrument, called the "reflectoscope", in which a pulse of ultrasonic waves was transmitted into the body under examination, and the time interval was measured when the reflected pulse returned.

The pulse echo method (Desch et. al., 1946) in which the same transducer transmits and receives the ultrasonic pulse was developed by Firestone (1940), Sokolov (1941), and Sproule (1945), and has become the most widely used system. A review of ultrasonic measurements of the location of defects in solids was reported by Doyle and Scala (1978), who developed the time-of-flight measurement method. From these early beginnings, ultrasonic testing has been developed into a highly sophisticated technology. It is used for assessing material properties and for flaw detection in many governmental organizations and private industry. The medical profession uses ultrasonic techniques for diagnostic and therapeutic purposes. In recent years, there is an increase in the use of ultrasonic techniques for material evaluation such as hardness, texture, and stress measurements, which is the main interest of this study.

\subsection{ACOUSTOELASTICITY}

Acoustoelasticity is the term applied to changes in velocity of a stress wave by applied or residual stress in materials. The change of stress wave velocities due to stress is induced by the higher order terms of the strain-displacement and constitutive relationships of 
materials (Murnaghan, 1951). The phenomenon of wave velocity variation with respect to stress increments has been observed and applied successfully in many fields, i.e., residual stress measurements (Allen and Sayers, 1984; Allison et.al., 1983; Binka and Sachse, 1976; Clark and Moulder, 1985; Dike and Johnson, 1990; Lu and Man, 1989; Pao et al., 1984) and bolt stress measurements (Allison and Clendenin, 1989; Bickford, 1988; Heyman and Chern, 1982; Johnson et al., 1986; Joshi and Pathare, 1984). A recent application was reported by Chen et al. (1998) for the stress measurement for post-tensioned prestressing rods used in a stressed timber bridge system. In this paper (Chen et. al., 1998), a stress wave was generated at one end of the prestressing rod and was detected at the other end using an ultrasonic transducer. Experimental results show that the traveling time of the stress wave through the rod was sensitive to its stress level. The change of the traveling time of the stress wave was used to predict the relative change of stress (or tensile force) in the rod.

A stress wave propagating in a long, elastic circular rod is a complex phenomenon since the system becomes dispersive and the wave is propagating with group velocity, which is a function of the rod's geometry and the propagating frequency. In this study, the geometry of the seven-wire strand is more complicated than the rod since it was fabricated with six wires wound tightly around a center wire. However, the investigation is limited to the stress wave propagation in the center wire in order to simplify the analyses of the wave behavior. The following chapter will describe experimental and theoretical studies that evaluate the tension levels in the prestressing strand by measuring the traveling time of a stress wave propagating through the entire length of the strand. The use of an appropriate signal processing tool together with numerical analyses will be illustrated. 


\section{CHAPTER 3}

\section{ACOUSTIC EMISSION MONITORING OF REINFORCED}

\section{CONCRETE STRUCTURES USING TWO-DIMENSIONAL SURFACE}

\section{WAVEGUIDES}

\subsection{OVERVIEW OF THE ACOUSTIC WAVEGUIDE SYSTEM}

Figure 3.1 shows a schematic drawing of a two-dimensional acoustic surface waveguide system. The waveguides are attached on the surface of a reinforced concrete floor slab at several designated joints with a specific grid interval. AE signals generated from the concrete will propagate on the surface of the slab and reach the joints. When AE signals arrive at the joint, they excite the waveguides (Figure 3.2). The wave motions will then propagate through the waveguides and be detected by sensors mounted at the ends of the waveguides. Because the joints are small, only few wave motions will dissipate back into the concrete slab (Chen and He, 2001). For this reason, AE signals can be transmitted a long distance. Several factors are critical to the success of this technique such as the transmissibility of the waveguide material, size of the joint, interval between the joints, and the number of joints the AE signal must traverse before it is detected by the AE sensors.

Wave motions in a waveguide are dispersive and can propagate in different modes, i.e. longitudinal, torsional, and transverse. As far as detectability of signals is concerned, only wave motions in the longitudinal direction are monitored in this study (see Appendix 
A). When the wave motions arrive at the joint, mode conversion, phase change, reflection, and transmission will occur. A portion of the signals will be transmitted through the joints and the rest will be reflected or dissipated into the concrete floor slab. The signal transmission efficiency (the amplitude ratio of the transmitted signal to the incident signal at a joint) is a key concern in designing the surface waveguides, which can be modeled as a circular rod with rigid joints mounted on elastic half-space. Theoretical analyses were conducted in order to investigate the wave propagation phenomenon in a rod bounded on elastic half-space through a rigid joint (Chen and He, 2001). The analysis shows that the transmission efficiency is a function of size and material properties of the waveguide, the joint size, and the incident wave motions.

\subsection{DESIGN OF SURFACE WAVEGUIDES}

The selected waveguide material must have low acoustic attenuation to have efficient signal transmission. Galvanized steel wire with $1 \mathrm{~mm}$ diameter (Chen et. al., 1994) was selected as a waveguide material in this study. The joint size is selected as the minimum size for a secured connection between the surface waveguides and the reinforced concrete slab's surface. Epoxy glue was used to build the joints, which can provide reliable contact. The size of an epoxy joint is approximately $5 \mathrm{~mm}$ in diameter and $3 \mathrm{~mm}$ in height. Selection of the joint interval of a surface waveguide system is the balance of the following. A small interval that can provides the shortest possible path between the concrete AE source and the

joint might be chosen because of the high attenuation in concrete. Or, on the other hand. a large interval might be chosen because of the amount of AE energy that would 
dissipate through each joint. Thus, an optimum interval of the joints should be determined. Attenuation of the tested concrete floor slab hardly allowed AE signals, which have major frequency components about $150 \mathrm{kHz}$, to travel farther than $30 \mathrm{~cm}$. In this study, a joint interval of $20 \mathrm{~cm}$ was chosen. This would allow a strong AE signal to arrive at each joint. It is worthwhile to note that typical $\mathrm{AE}$ signals from concrete cracking with a frequency range between $100 \mathrm{kHz}$ to $300 \mathrm{kHz}$ can be expected (Otshu 1987, Chen et al 1992). The lower frequency components of the AE signals could propagate farther away in concrete than the high frequency components but might be noisy.

As shown in Figure 3.3, three types of joints are categorized by the number of branches of the waveguides at each joint. These are 2, 3, and 4-branched joints. However, as far as the transmission directions of the $\mathrm{AE}$ signals at these joints are concerned, they can be categorized into 5 cases as illustrated in Figure 3.3. In case A, after an AE signal arrives at the joint, energy of the signal will only be transmitted into the same wire. In transmission cases B, C, D and E, some part of the energy will be transmitted into the same wire and some will be transmitted into the other wires.

\subsection{INSTRUMENTATION}

\section{LOCAN-AT}

A six-channel acoustic emission system (LOCAN-AT, Physical Acoustic Corporation, PAC) was used in this study. The LOCAN-AT is a computerized acoustic emission system that performs AE signal measurements and stores, displays and analyzes the AE data. There are total of six monitoring channels in the LOCAN-AT. Each channel 
requires its own $\mathrm{AE}$ sensor, preamplifier, and connecting cables. AE data was first acquired by each channel independently. Then, all of the data was transferred to the main processor of the computer. For each channel, the data acquisition procedures are as follows:

1) converting $\mathrm{AE}$ signals into electrical signals by the $\mathrm{AE}$ sensor

2) amplifying signals to useable voltage levels by the preamplifier, and

3) digitalizing the signals in a computerized module.

Processing of acoustic emission signals is normally conducted in both time and frequency domains. Time domain signal processing involves extraction of burst type signal parameters such as events, peak amplitude, event duration, ringdown counts, and rise time. In the frequency domain, amplitudes of various components of an acoustic emission signal are computed and are usually presented in the form of frequency spectra. A threshold level is defined as the system's output amplitude corresponding to a signal level that is set by a user. Background noise is eliminated during an acoustic emission data acquisition by filter out those signals that have their peaks amplitude levels lower than the system's threshold.

As seen in Figure 3.4, an acoustic emission event is defined as the entire wave packet from the beginning of the threshold crossing to the end where the signal's peak amplitude drops just below the threshold level. Ringdown count is defined as the total number of oscillations crossing the threshold level in an event. Event duration is defined as the elapsed time between the first and the last oscillations crossing the threshold level. Rise time for the event is the elapsed time from the first oscillation crossing the threshold level to the time that 
is corresponding to the peak amplitude of the signal. The peak amplitude is defined as the maximum amplitude level of the oscillation that is measured with respect to the base line. Energy level of an event is defined as a measure of the area bounded by the threshold level and a line connecting the peaks of all oscillations that are located above the threshold level.

\section{$\underline{\text { Sensors and Preamplifiers }}$}

A total of six PICO acoustic emission sensors (PAC) were used in this study. One sensor was used for each channel. The sensor is $4.2 \mathrm{~mm}$ in diameter and $4.0 \mathrm{~mm}$ in height. The operating frequency range is between $100 \mathrm{kHz}$ and $1000 \mathrm{kHz}$. The calibration curve of the sensor is shown in Figure 3.5.

One PAC-1220 preamplifier was used for each channel. The preamplifier was connected to the sensor. It amplified and transfered AE signals to the LOCAN-AT. Each preamplifier can provide a $40 \mathrm{~dB}$ or $60 \mathrm{~dB}$ gain ( $40 \mathrm{~dB}$ gain was used in this study). The preamplifier is also featured with a $100 \mathrm{kHz}$ to $1200 \mathrm{kHz}$ band-pass filter.

\subsection{TRANSMISSION EFFICIENCIES OF AE SIGNALS AT EPOXY JOINTS}

Figure 3.6 - Figure 3.10 show experimental setup for determining the transmission efficiencies of the AE signals in 5 directions at the epoxy joints. In this experiment, surface waveguides were mounted on the surface of concrete beams. The waveguides were arranged into 5 different formations corresponding to each transmission case. Two AE sensors were attached to the ends of the waveguide in the longitudinal direction using hotmelt glue. Each sensor was connected to the preamplifier. The sensor's small size allowed 
it to be easily attached to the waveguide. To generate AE signals, a pencil-lead break, as an artificial $\mathrm{AE}$ source, was performed at every joint location on the concrete surface. The pencil lead, manufactured by Pentel, was $0.5 \mathrm{~mm}$ in diameter. A plastic break shoe was used to control the pencil-lead break. Peak amplitudes of the detected AE signals were recorded by the LOCAN-AT system. A sampling rate of $4 \mathrm{MHz}$ was used. A threshold level of $38 \mathrm{~dB}$ was set on the LOCAN-AT system to filter out the background noise.

In determining the attenuation of $\mathrm{AE}$ signals on the surface of concrete, two sensors were mounted $30 \mathrm{~cm}$ apart on the concrete surface using hot-melt glue as a coupling agent (Figure 3.11). The standard pencil-lead break was then performed on the concrete surface at every $5 \mathrm{~cm}$ between the two sensors. Peak amplitudes of the detected AE signals were recorded by the LOCAN-AT system.

Consider an AE signal (with original peak amplitude, $\mathrm{A}_{0}$ ) propagates over a distance $x$ on the surface of the reinforced concrete floor slab, reaches a joint, and then propagates in the waveguide through $n$ joints. The detected peak amplitude at sensor 1 is called $\mathrm{A}_{1}$ and can be expressed as

$$
\mathrm{A}_{1}=\mathrm{A}_{0} \mathrm{k}^{\mathrm{n}} \mathrm{e}^{-\alpha \mathrm{x}}
$$

where $k$ is the transmission efficiency of the joint and $\alpha$ is the attenuation rate of concrete.

In the first experiment, the pencil-lead breaks were conducted at the joints so that the exponential term can be eliminated. For the same signal traveling in the opposite direction (Figure 3.12), the peak amplitude detected at sensor 2 is represented by $A_{2}$. Note that in equation (3.1), the attenuation in the steel wire is assumed negligible in comparison to the attenuation of concrete and the epoxy joints. 
Since pencil-lead breaks performed at different events may generate different peak amplitude $\left(\mathrm{A}_{0}\right)$, by using AE sensors simultaneously, $\mathrm{A}_{0}$ can be eliminated. The ratio of the peak amplitudes received by the two sensors located at the ends of the waveguide can be written as:

$$
\frac{A_{1}}{A_{2}}=\frac{k^{n}}{C k^{(N-n+1)}}
$$

where $n$ is the number of joints that the AE signal has to pass to reach Sensor 1 (Figure 3.12), $C$ is the sensitivity ratio between the two sensors, and $N$ is the total number of joints $(N=5)$. By capturing $\mathrm{AE}$ signals with these sensors, the transmission efficiency, $k$, can be experimentally obtained.

Figure 3.13 shows the plots of attenuation curves presented by the amplitude ratio versus traveling distance. The isolated curve to the left represents the amplitude ratio of the AE signals propagating on concrete surface (no waveguide was used). The group of curves to the right represents the amplitude ratio of the $\mathrm{AE}$ signals propagating in the steel waveguides for all transmission cases. The slopes of these curves can be used to determine the severity of signal attenuation. It is shown that the reduction of the amplitude ratio $\left(A_{1} / A_{2}\right)$ of the $A E$ signals propagating on concrete surface is at a much higher rate than those propagating in the surface waveguides. From the experimental results (Figure 3.13), transmission efficiencies were calculated as $k=0.92,0.90,0.89,0.87$, and 0.85 in case A, B, $\mathrm{C}, \mathrm{D}$, and E, respectively. The results indicate that (1) transmission efficiencies of the AE signals at the joints are relatively high, which indicates a small energy loss at each joint, and (2) a greater reduction of peak amplitude (or energy) occurs when AE signals transmit into a different wire (case D and E). 


\subsection{FREQUENCY COMPONENTS OF AE SIGNALS}

An experiment was conducted in order to investigate the nature of the AE signals generated by the pencil-lead break. In this study, pencil-lead breaks were conducted adjacent to the AE sensor that is located on the concrete surface (Figure 3.14). The AE signals were recorded by the MISTRAS system. Figure 3.15 shows one example of the AE signals presented in time domain. The generated signal is a burst-type signal, which is similar to the typical AE signals detected from concrete (Maji and Shah, 1988). By applying the Fourier transform technique, frequency components of this signal are illustrated in Figure 3.16. In this figure, major frequency components between $100 \mathrm{kHz}$ and $300 \mathrm{kHz}$ are observed. The peak frequency component of this signal is about $150 \mathrm{kHz}$. It is noted that frequency components in this range can be expected from typical AE signals generated by crack growth in concrete (Ohtsu, 1987). Therefore, the pencil-lead break method is an appropriate method for producing AE signals in this study.

Figure 3.17 shows a burst-type AE signal recorded by the sensor located at one end of the waveguide that was mounted with the concrete surface (transmission case A, Figure 3.6). This AE signal is a result of conducting a pencil-lead break on concrete surface at the other end of the waveguide. Figure 3.18 shows that frequency components of this signal are in the same range as the one generated from the original pencil-lead break on concrete surface (Figure 3.16). Therefore, the waveguide system is sensitive to the frequency components between $100 \mathrm{kHz}$ to $300 \mathrm{kHz}$ so that it can possibly be used to detect the actual AE signals in concrete. 


\subsection{AE SOURCE LOCATIONS}

In order to identify AE source locations, experiments on the 2-D acoustic surface waveguide system (Figure 3.1) were conducted. The 2-D acoustic waveguide system was attached on the surface of a reinforced concrete floor slab of $4 \frac{3}{4}$ inch thickness. Six AE sensors were used to record artificial AE signals generated from standard pencil-lead breaks at various locations on the concrete surface. As shown in Figure 3.19, each pencil-lead break location is represented by the cartesian coordinate system $(\mathrm{x}, \mathrm{y})$. The length unit is in meter. At each pencil-lead break event, several AE parameters were recorded.

Due to reflection, dissipation, and transmission of energy into different directions at the joint, occasionally the $\mathrm{AE}$ signals could not reach all six $\mathrm{AE}$ sensors at one particular pencil-lead break event. Figure 3.20 shows an example of the AE waveforms detected at four $\mathrm{AE}$ sensors due to a pencil-lead break at coordinate $(1.2,0.4)$. Results shows that sensor 5 was the first sensor that could detect the AE signals follows by sensor 1 , sensor 4 , and sensor 2, respectively. Due to the complexity of the waveguide system, there is a possibility that an AE signal could propagate from its source into different routes on the waveguide mesh and recombine in some very complicated way before reaching the sensors. From the earlier described transmission cases, signals propagating in different routes on the waveguide system will lose different amount of energy depending on the traveling distances, number of joints and the propagation directions of the signals. The AE signal that propagates in the most efficient route will lose the lowest amount of energy and will dominantly constitute in the recorded waveform. Using this assumption, the most efficient routes of the AE signals 
from the source to the sensors can be identified. Figure 3.21 shows the propagation routes of signals generated at coordinate $(1.2,0.4)$. It is clear that sensor number 5 will be the first sensor to detect the signal because of its shortest distance from the AE source followed by sensor number 1,4 , and 2. In the same order, experimental results show that the AE signal detected by sensor 5 has the highest peak amplitude followed by the signals detected by the rest of the sensors, which propagate through a greater number of joints. Understanding the propagation routes of the $\mathrm{AE}$ signals, the joint's transmission efficiencies can be used to estimate how far the AE signals would propagate. This will benefit the design of the $\mathrm{AE}$ monitoring area.

Figure 3.21 also shows peak amplitude ratios of the detected AE signals. Since sensor 5 recorded the highest peak amplitude, it is convenient to use this amplitude as a numerator for calculating all other peak amplitude ratios. Accordingly, $\left(\mathrm{A}_{5} / \mathrm{A}_{1}\right)=1.14$, $\left(A_{5} / A_{4}\right)=1.35$, and $\left(A_{5} / A_{2}\right)=1.70$. These peak amplitude ratios show their repeatability. It means that the peak amplitude ratios remain constant regardless of the variation of input amplitude $\left(\mathrm{A}_{0}\right)$ from different pencil-lead break events.

From the previous study illustrated in Figure 3.6 - Figure 3.10, the transmission efficiency in each case was predetermined. Since there could be up to 5 different transmission cases in each route, equation (3.1) is modified as

$$
\mathrm{A}_{\mathrm{i}}=\mathrm{A}_{0}\left(\mathrm{k}_{1}^{\mathrm{n}_{1}} \mathrm{k}_{2}^{\mathrm{n}_{2}} \mathrm{k}_{3}^{\mathrm{n}_{3}} \mathrm{k}_{4}^{\mathrm{n}_{4}} \mathrm{k}_{5}^{\mathrm{n}_{5}}\right) \mathrm{e}^{-\alpha \mathrm{x}}
$$

where $A_{i}$ represents receiving peak amplitude at sensor $i(i=1,2,3,4,5,6)$. 
Since the propagation routes were identified, the number of joints, $\mathrm{n}_{\mathrm{j}}$, and their corresponding transmission cases, $\mathrm{k}_{\mathrm{j}}$, in each route can be determined. By substituting these numbers in equation (3.3), amplitude ratios can be predicted as $\left(\mathrm{A}_{5} / \mathrm{A}_{1}\right)=1.25,\left(\mathrm{~A}_{5} / \mathrm{A}_{4}\right)=1.46$, and $\left(A_{5} / A_{2}\right)=2.20$. The result shows that the predicted peak amplitude ratios $\left(A_{5} / A_{1}\right)$ and $\left(\mathrm{A}_{5} / \mathrm{A}_{4}\right)$ have good agreement with the ones from the experiment. However, the peak amplitude ratio $\left(\mathrm{A}_{5} / \mathrm{A}_{2}\right)$ shows a larger error. This is probably due to the variation of the joints of each type. Even though the joints of the same type were constructed using the same procedures, they could not be identical. A longer propagation route with a greater number of joints would produce a larger error of the peak amplitude ratio. In addition, the possibility of recombining $\mathrm{AE}$ signals from different propagation routes may also have an effect on the peak amplitude.

In practical cases, cracks can happen virtually anywhere on the reinforced concrete floor slab. This represents an indefinite number of possible AE source locations. The propagation routes of the $\mathrm{AE}$ signals can be very complicated so that the $\mathrm{AE}$ 's data interpretation can be much more difficult than the case presented. Also, boundary conditions and environmental effects may create variations of AE signal characteristics. For this reason, a NN system was used to predict locations of the AE sources. 


\subsection{NEURAL NETWORK}

\subsubsection{AE Source Locations on the Reinforced Concrete Floor Slab}

Using the same experimental set-up and instrumentation, the standard pencil-lead breaks were performed at forty-nine locations on the reinforced concrete floor slab (Figure 3.19). Forty locations are very close to the epoxy joints, which attached the waveguides to the slab's surface. The remaining nine locations are in the middle of the waveguide mesh (intermediate locations), for example, at coordinate $(0.2,0.2)$. AE signals generated at these intermediate locations need to travel some distance on the concrete surface to reach the joints. The LOCAN-AT system was used to record AE parameters. These AE parameters were peak amplitude, energy, and the order of the sensors that detect the AE signals. A schematic diagram of the experiment is shown in Figure 3.22.

\subsubsection{Network Architecture}

A multi-layer NN system (Chen, 1992) consists of a set of highly interconnected, nonlinear processing units (PU's) operating in parallel. Each connection between two PU's carries an adjustable, continuous value called weight, which can be positive (excitatory), negative (inhibitory), or zero. A 3-layer NN system (Hornik, 1991) was used in this study. The Back Propagation (BP) was used as a learning algorithm of the NN. The network, as shown in Figure 3.23, consists of the input layer, hidden layer, output layer, and inter-layer connections. The input layer was arranged into a 2-dimensional plane representing a pattern 
of $\mathrm{AE}$ parameters from each pencil-lead break at each location on the reinforced concrete slab's surface. The input plane consists of 18 ( $6 \mathrm{AE}$ sensors $\times 3 \mathrm{AE}$ parameters) input units that were transferred to the hidden units through the connections. Each input unit is fully connected to all the hidden units.

The number of hidden units in the hidden layer can be arbitrary. With a given training data set, determining the optimal number of hidden units in advance is difficult for the BP type algorithm. A trial and error method, which is simple and straight forward, was therefore applied. The hidden layer forms an internal representation in order to provide correct outputs. The desired output is the coordinate $(\mathrm{x}, \mathrm{y})$ of the pencil-lead break location.

Each PU in the hidden layer and the output layer computes total input from summation of the inputs and weights by

$$
\text { net }_{\mathrm{j}}=\sum_{\mathrm{i}} \mathrm{w}_{\mathrm{ij}} \mathrm{y}_{\mathrm{i}}+\theta_{\mathrm{j}}
$$

where net $t_{j}$ denotes the total input to $\mathrm{PU}_{\mathrm{j}} ; \mathrm{w}_{\mathrm{ij}}$ is the weight of the connection between $\mathrm{PU}_{\mathrm{i}}$ and $\mathrm{PU}_{\mathrm{j}} ; \mathrm{y}_{\mathrm{i}}$ is the actual output of $\mathrm{PU}_{\mathrm{i}}$; and $\theta_{\mathrm{j}}$ is the bias of $\mathrm{PU}_{\mathrm{j}}$. The total input net transformed through a nonlinear, differentiable sigmoid function (Rumelhart et al., 1986) to generate the actual output. The output coordinates range from $(0.0,0.0)$ to $(1.2,1.2)$. For each PU in the network, the output function was given as

$$
y_{j}=f\left(\text { net }_{j}, U_{j}, L_{j}, T_{j}\right)=\frac{U_{j}-L_{j}}{1+e^{- \text {net }_{j} / T_{j}}}+L_{j}
$$


where $U_{j}$ and $L_{j}$ represent the upper bound and the lower bound of the output function, respectively, and $T_{j}$ represents the slope of the function.

\subsubsection{Training and Testing of NN}

Training is a process that adjusts the connection weights according to a learning procedure. The adjustment is processed by introducing a set of training patterns to the network. When the training process converges, the network achieves the required mapping, and the weights would represent knowledge that the network has acquired. In practical cases, a trained network not only performs correct responses on the training patterns, but also on the untrained patterns as well. The objective of this study is to train a network that is able to predict the locations of the the $\mathrm{AE}$ sources on reinforced concrete floor slabs. The BP technique was used for training the multi-layer network.

Prediction performance of the network is evaluated by Correct Rate (CR) and Root Mean Square (RMS) value. These are defined as

$$
\mathrm{CR}=\frac{(\text { Number of patterns correctly classified })}{(\text { Total number of patterns of the data sets })} \times 100 \%
$$

and

$$
\mathrm{RMS}=\frac{1}{\mathrm{P}} \sum_{\mathrm{i}=1}^{\mathrm{P}}\left[\frac{1}{\mathrm{~m}} \sum_{\mathrm{j}=1}^{\mathrm{m}} \sqrt{\left(\mathrm{d}_{\mathrm{j}}^{(\mathrm{i})}-\mathrm{y}_{\mathrm{j}}^{(\mathrm{i})}\right)^{2}}\right]
$$


where $\mathrm{P}$ is the total number of training patterns, $\mathrm{m}$ is the number of output units, $\mathrm{d}_{\mathrm{j}}$ is the predicted output, and $\mathrm{y}_{\mathrm{j}}$ is the actual output. Convergence of the training procedure is satisfied by (1) $100 \%$ correct rate on the training data set, or (2) 0.05 or lower for Root Mean Square (RMS) value of the training data set. When either condition is satisfied, the training procedure converges.

\subsubsection{NN Results and Discussions}

A total of 5 data sets were recorded in this study. Each data set contains forty-nine data points corresponding to the forty-nine pencil-lead break locations on the reinforced concrete floor slab (Figure 3.19). Four data sets were used as training data sets. The remaining data set was used as a testing data set. The $\mathrm{NN}$ simulations were conducted on a personal computer. All simulations converged after the RMS value from the training data sets reduced to 0.05 . The number of hidden units was determined by training the $\mathrm{NN}$ using different numbers of hidden units. Ten hidden units were chosen in the NN because they provided the highest correct rates for the training data sets. The NN was trained and terminated at 19,500 cycles when the RMS value of the training data sets was 0.05 . The correct rates in the $\mathrm{x}$-direction and the $\mathrm{y}$-direction for the training data sets with 10 hidden units were $86.2 \%$ and $87.8 \%$, respectively. The weights were established and were used to

predict the coordinates of the artificial AE sources from the testing data set. As shown in Figure 3.24, prediction results from the testing data set show $79.6 \%$ correct rate in the $\mathrm{x}$ direction, $87.8 \%$ correct rate in the y-direction with 10 hidden units. In addition, when the NN was used to predict AE source locations of the testing data set, the lowest RMS was also 
obtained from using ten hidden units (Figure 3.25, RMS value $=0.058$ ). The prediction results are shown in Table 3.1. The corresponding Figure 3.26 shows graphical results from the NN prediction. The actual pencil-lead break locations from the testing data set are designated at coordinate $(\mathrm{x}, \mathrm{y})$, where $\mathrm{x}$ ranges from $0.0 \mathrm{~m}$ to $1.2 \mathrm{~m}$ and $\mathrm{y}$ ranges from $0.0 \mathrm{~m}$ to $1.2 \mathrm{~m}$. For each location, a straight line is used to connect the markers of the actual and the predicted locations.

Figure 3.27 shows an error distribution of the NN prediction. From the forty-nine predicted locations, fifteen predicted locations $(30.6 \%)$ have less than $5 \mathrm{~cm}$ error, sixteen predicted locations $(32.7 \%)$ have errors in the 5 to $10 \mathrm{~cm}$ range, and eleven predicted locations $(22.4 \%)$ have errors in the 10 to $15 \mathrm{~cm}$ range. The average error of the forty-nine predicted locations is $9.28 \mathrm{~cm}$. An average error of the forty predicted locations near the epoxy joints is $9.23 \mathrm{~cm}$. The remaining nine predicted intermediate locations on the concrete surface have the average error of $9.51 \mathrm{~cm}$. It is clear that at this accuracy level, the use of the acoustic surface waveguide system along with the NN system can effectively locate the AE sources on the reinforced concrete slab.

\subsection{SUMMARY}

The development of a two-dimensional acoustic surface waveguide system to detect acoustic emission activities of a reinforced concrete slab was presented. High signal transmission efficiencies of the waveguide joints were obtained. The experimental results

show that the two-dimensional surface waveguides can significantly increase the AE monitoring range. A neural network system was employed to predict the AE source locations on the reinforced concrete slab. The results of the neural networks show 
acceptable prediction accuracy in locating the AE sources. The proposed surface waveguide system can be easily attached on the surface of existing structures made of high attenuation materials such as concrete or fiber-reinforced composites for damage assessment. 


\begin{tabular}{|c|c|c|c|c|}
\hline \multicolumn{2}{|c|}{$\begin{array}{c}\text { Pencil-lead Break } \\
\text { Location }\end{array}$} & \multicolumn{2}{|c|}{$\begin{array}{l}\text { Predicted } \\
\text { Location }\end{array}$} & \multirow{2}{*}{$\begin{array}{c}\text { Prediction } \\
\text { Error } \\
(\mathrm{m})\end{array}$} \\
\hline $\mathrm{X}(\mathrm{m})$ & $\mathrm{Y}(\mathrm{m})$ & $\mathrm{Xp}(\mathrm{m})$ & $Y p(m)$ & \\
\hline 0.00 & 0.00 & 0.03 & 0.02 & 0.04 \\
\hline 0.20 & 0.00 & 0.21 & 0.01 & 0.01 \\
\hline 0.40 & 0.00 & 0.27 & 0.01 & 0.13 \\
\hline 0.60 & 0.00 & 0.41 & 0.04 & 0.19 \\
\hline 0.80 & 0.00 & 0.83 & 0.08 & 0.09 \\
\hline 1.00 & 0.00 & 1.02 & 0.01 & 0.02 \\
\hline 1.20 & 0.00 & 1.19 & 0.03 & 0.03 \\
\hline 0.00 & 0.20 & 0.04 & 0.21 & 0.04 \\
\hline 0.20 & 0.20 & 0.14 & 0.14 & 0.08 \\
\hline 0.40 & 0.20 & 0.40 & 0.24 & 0.04 \\
\hline 0.60 & 0.20 & 0.55 & 0.13 & 0.09 \\
\hline 0.80 & 0.20 & 0.83 & 0.18 & 0.04 \\
\hline 1.00 & 0.20 & 1.05 & 0.22 & 0.05 \\
\hline 1.20 & 0.20 & 1.19 & 0.21 & 0.01 \\
\hline 0.00 & 0.40 & 0.10 & 0.32 & 0.13 \\
\hline 0.20 & 0.40 & 0.17 & 0.45 & 0.06 \\
\hline 0.40 & 0.40 & 0.37 & 0.38 & 0.04 \\
\hline 0.60 & 0.40 & 0.61 & 0.69 & 0.29 \\
\hline 0.80 & 0.40 & 0.86 & 0.31 & 0.11 \\
\hline 1.00 & 0.40 & 0.91 & 0.43 & 0.09 \\
\hline 1.20 & 0.40 & 1.14 & 0.38 & 0.06 \\
\hline 0.00 & 0.60 & 0.10 & 0.63 & 0.10 \\
\hline 0.20 & 0.60 & 0.23 & 0.62 & 0.04 \\
\hline 0.40 & 0.60 & 0.39 & 0.62 & 0.02 \\
\hline 0.60 & 0.60 & 0.69 & 0.61 & 0.09 \\
\hline 0.80 & 0.60 & 0.88 & 0.48 & 0.14 \\
\hline 1.00 & 0.60 & 0.95 & 0.84 & 0.25 \\
\hline 1.20 & 0.60 & 1.15 & 0.62 & 0.05 \\
\hline 0.00 & 0.80 & 0.03 & 0.73 & 0.08 \\
\hline 0.20 & 0.80 & 0.21 & 0.98 & 0.18 \\
\hline 0.40 & 0.80 & 0.47 & 0.81 & 0.07 \\
\hline 0.60 & 0.80 & 0.74 & 0.83 & 0.14 \\
\hline 0.80 & 0.80 & 0.75 & 0.81 & 0.05 \\
\hline 1.00 & 0.80 & 1.03 & 0.74 & 0.07 \\
\hline 1.20 & 0.80 & 1.17 & 0.76 & 0.05 \\
\hline 0.00 & 1.00 & 0.01 & 0.99 & 0.01 \\
\hline 0.20 & 1.00 & 0.22 & 1.03 & 0.04 \\
\hline 0.40 & 1.00 & 0.53 & 1.06 & 0.14 \\
\hline 0.60 & 1.00 & 0.63 & 1.05 & 0.06 \\
\hline 0.80 & 1.00 & 0.83 & 0.88 & 0.12 \\
\hline 1.00 & 1.00 & 0.96 & 0.84 & 0.16 \\
\hline 1.20 & 1.00 & 1.17 & 0.95 & 0.06 \\
\hline 0.00 & 1.20 & 0.23 & 1.19 & 0.23 \\
\hline 0.20 & 1.20 & 0.34 & 1.19 & 0.14 \\
\hline 0.40 & 1.20 & 0.29 & 1.12 & 0.14 \\
\hline 0.60 & 1.20 & 0.57 & 1.17 & 0.04 \\
\hline 0.80 & 1.20 & 1.00 & 1.14 & 0.21 \\
\hline 1.00 & 1.20 & 1.12 & 1.14 & 0.13 \\
\hline 1.20 & 1.20 & 1.14 & 1.16 & 0.07 \\
\hline & & Ave & rror & 0.09 \\
\hline
\end{tabular}

Table 3.1 Comparison between actual and predicted pencil-lead break locations 


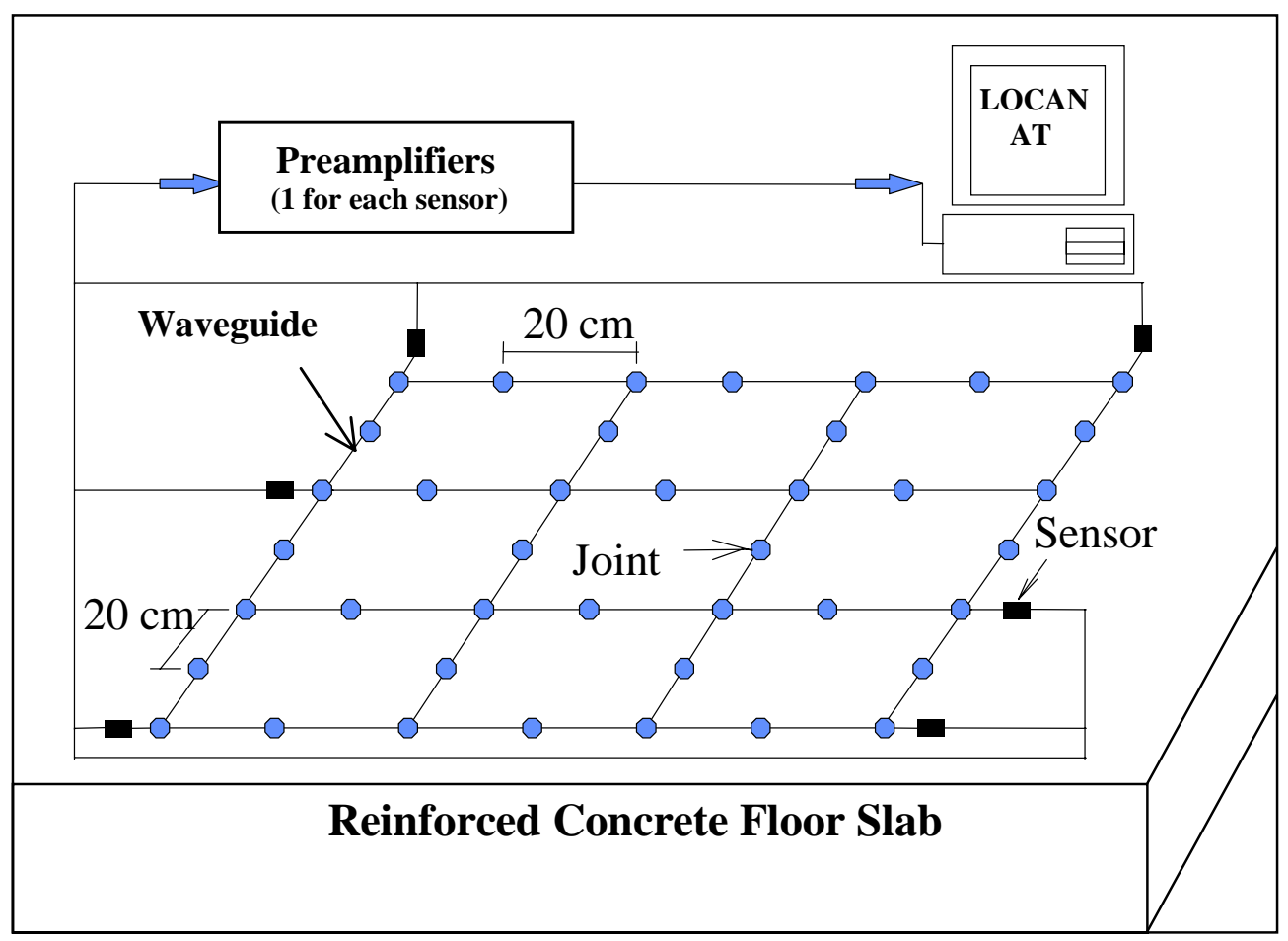

Figure 3.1 Experimental setup and instrumentation 


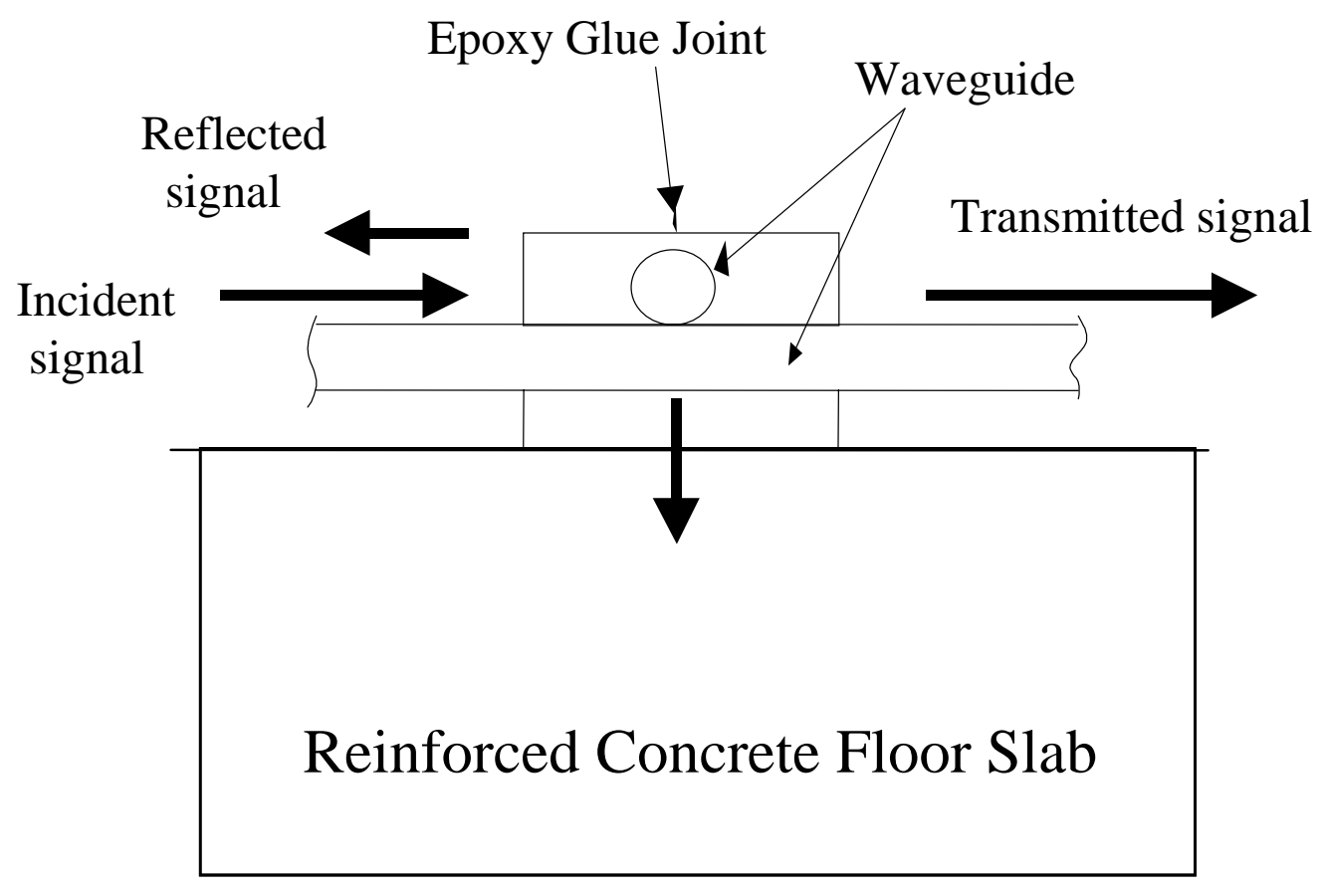

Figure 3.2 Wave propagation at an epoxy joint 

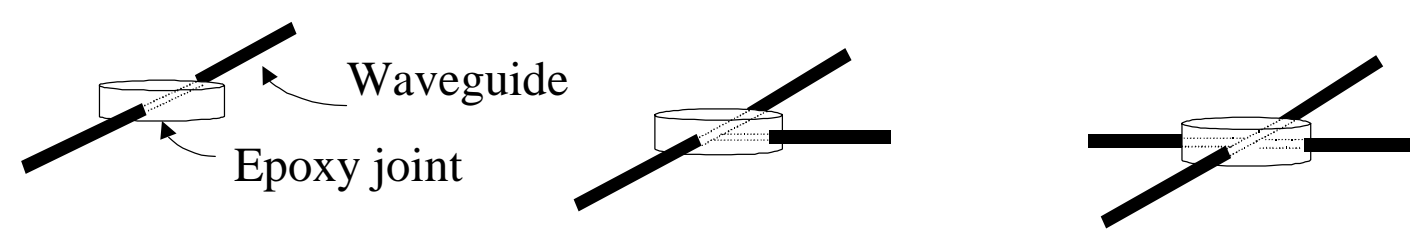

$\bullet$
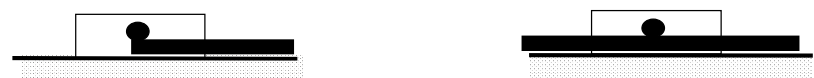

Reinforced concrete slab
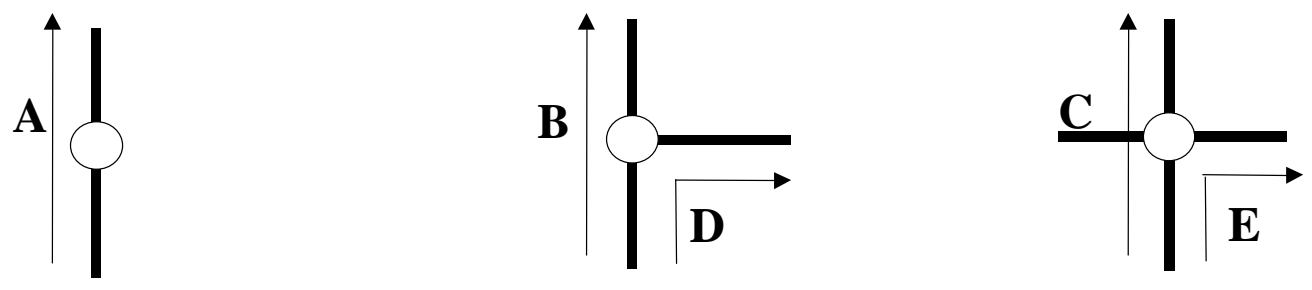

Figure 3.3 Transmission directions of AE signals at epoxy joints 

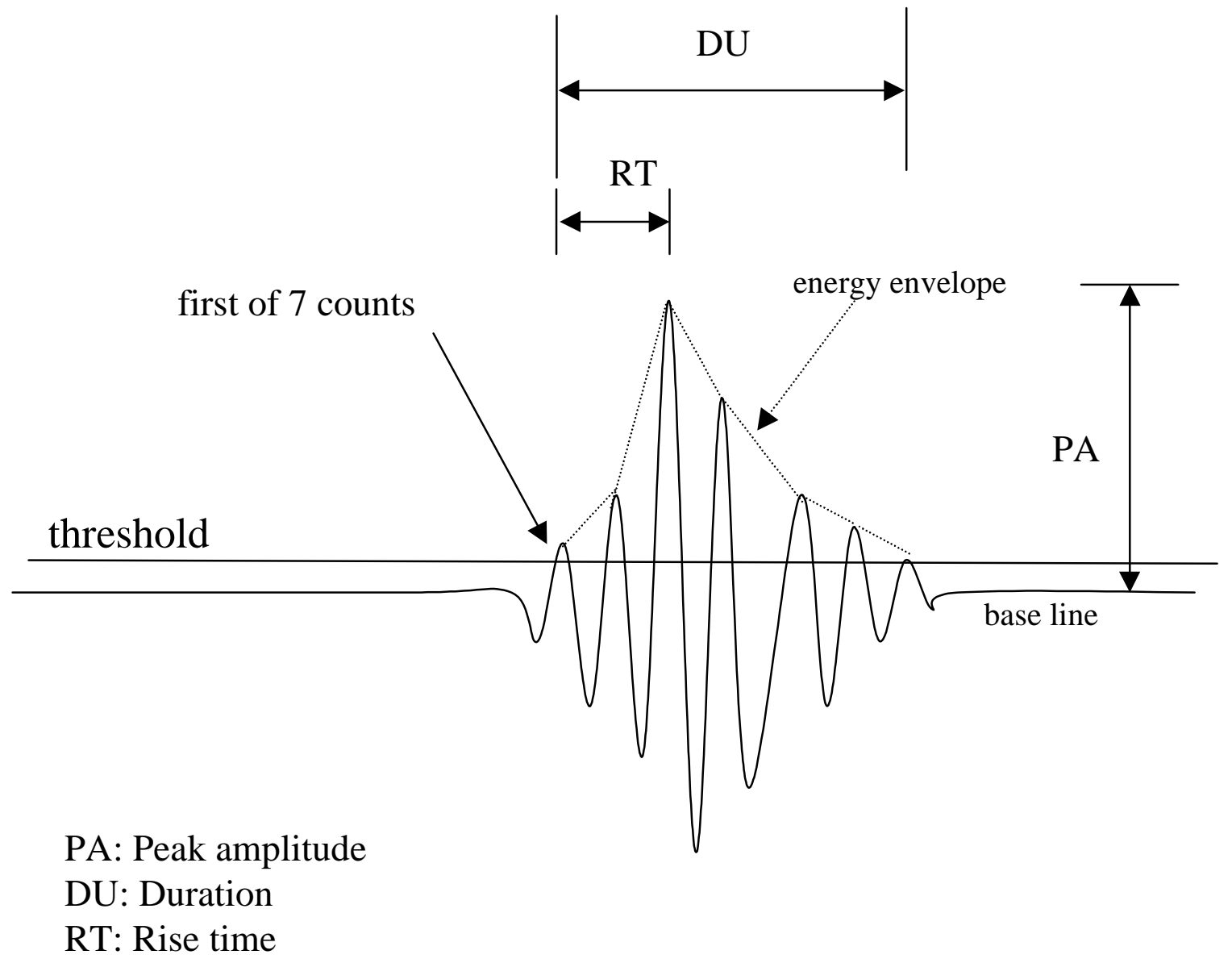

Figure 3.4 AE parameters 


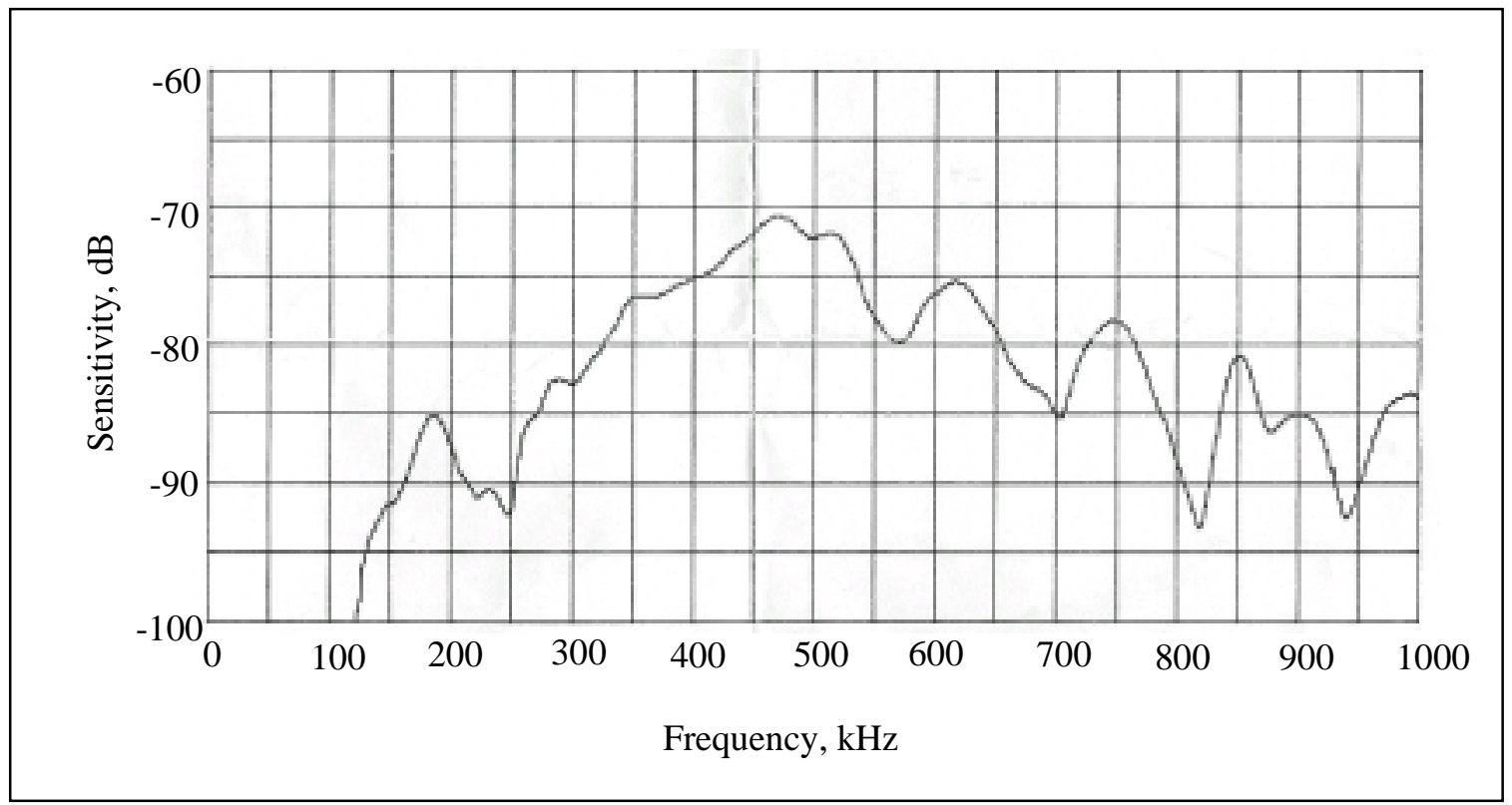

Figure 3.5 Calibration chart of the AE sensor 


\section{CASE A}

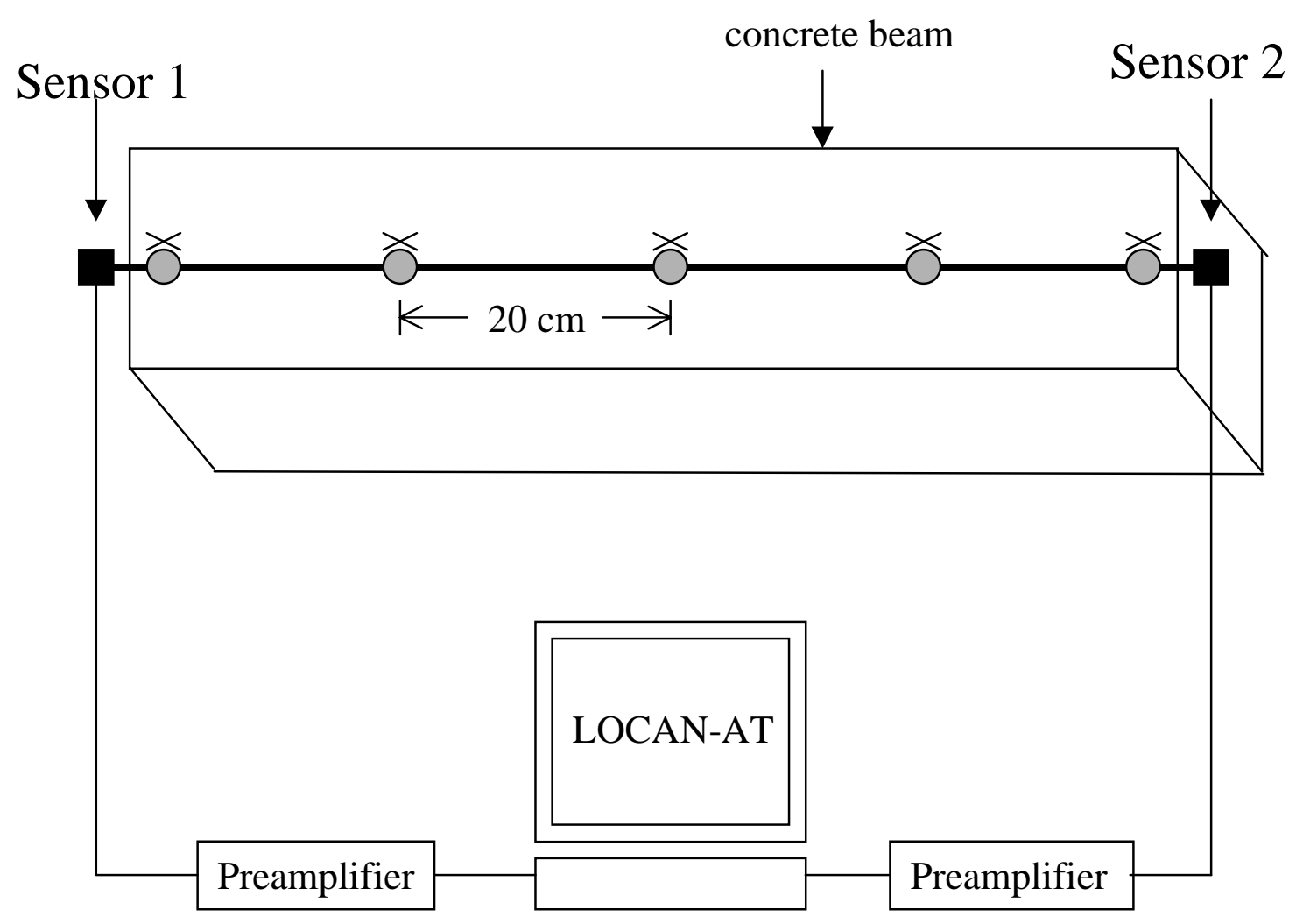

— PICO sensor

$X \quad$ artificial AE source (pencil-lead break)

waveguide joint

Figure 3.6 Experimental setup for transmission efficiency determination of surface waveguides (Case A) 


\section{CASE B}

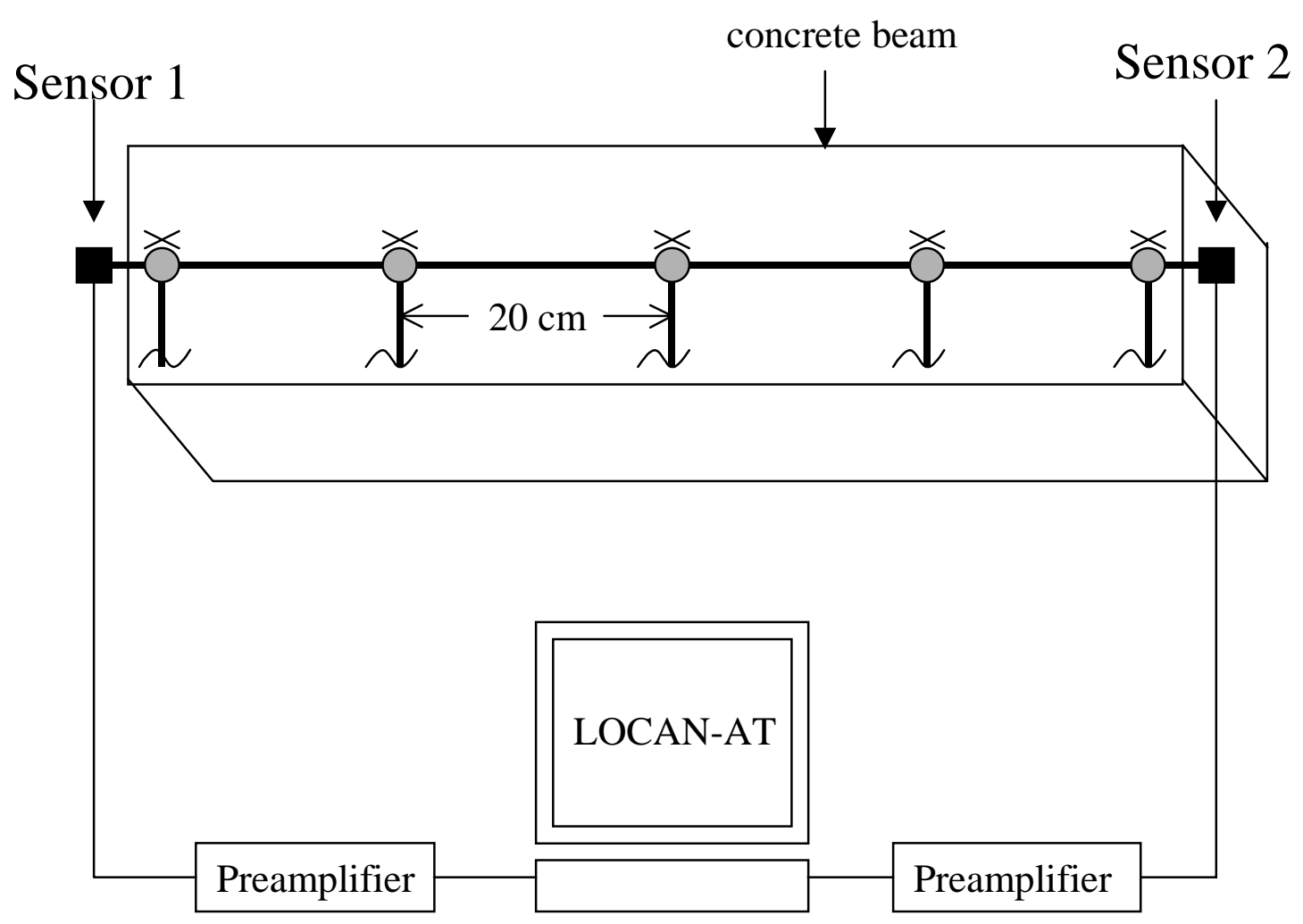

\footnotetext{
- PICO sensor

X artificial AE source (pencil-lead break)

waveguide joint
}

Figure 3.7 Experimental setup for transmission efficiency determination of surface waveguides (Case B) 


\section{CASE C}

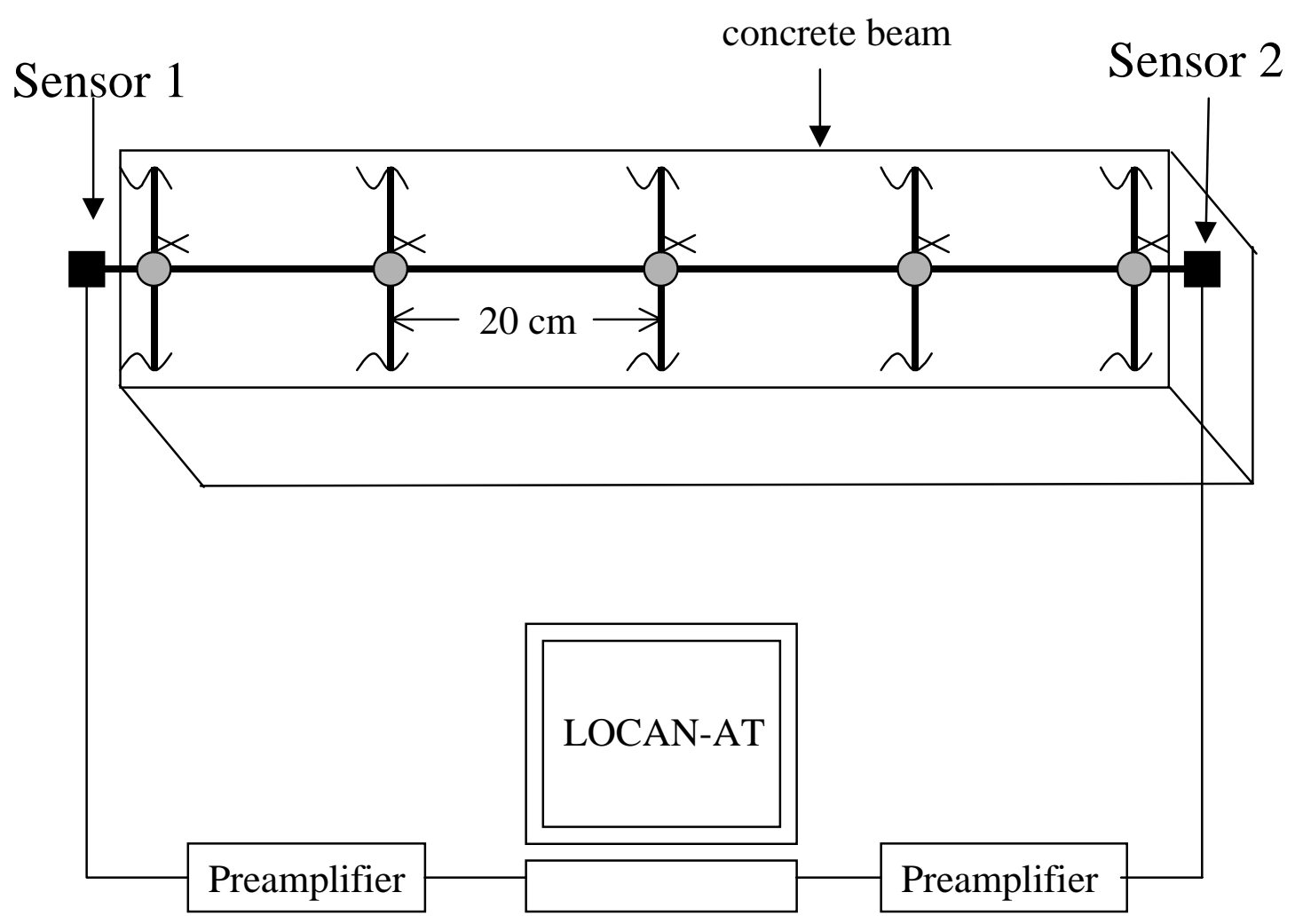

Figure 3.8 Experimental setup for transmission efficiency determination of surface waveguides (Case C) 


\section{CASE D}

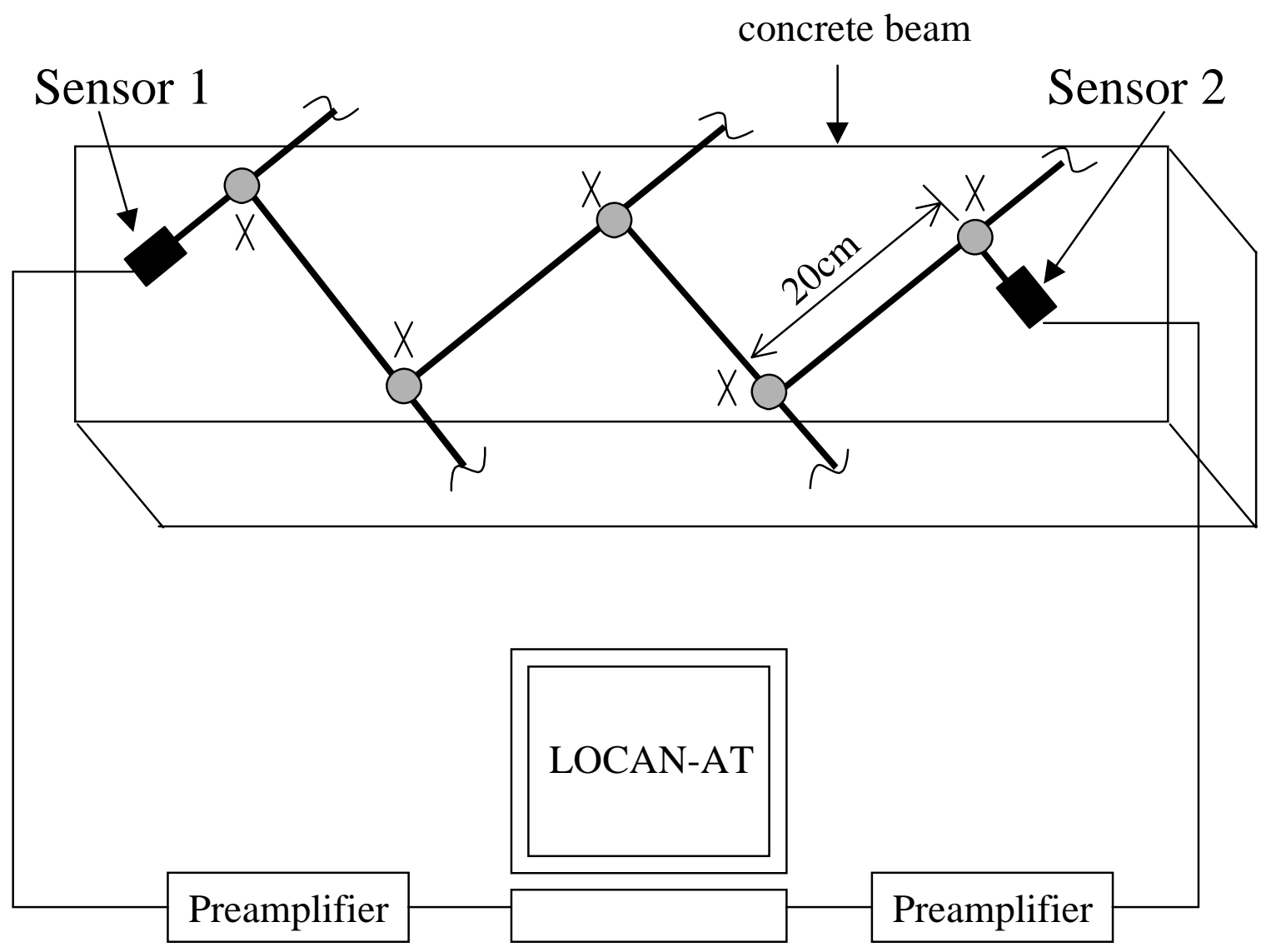

Figure 3.9 Experimental setup for transmission efficiency determination of surface waveguides (Case D) 


\section{CASE E}

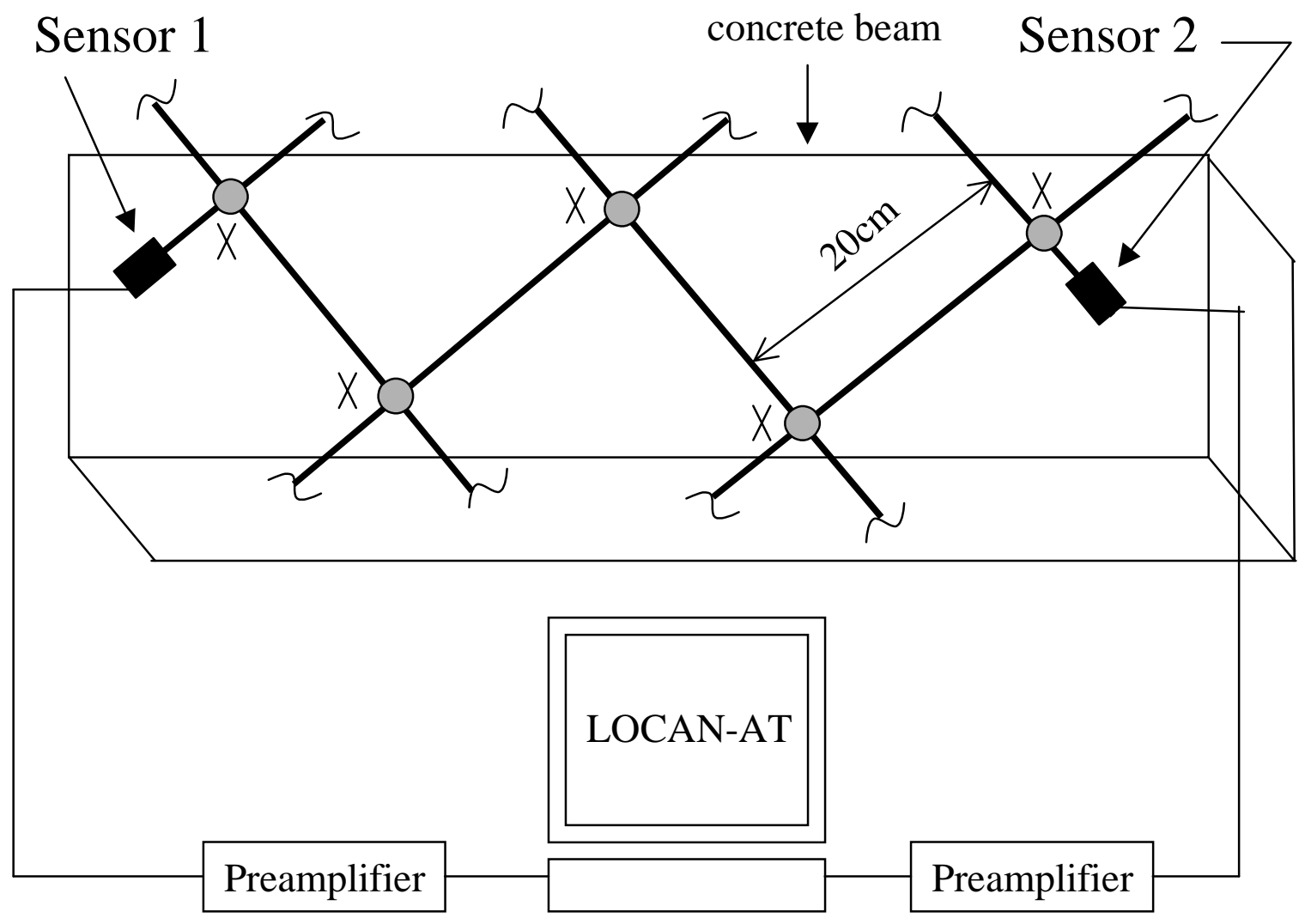

- PICO sensor

$X \quad$ artificial AE source (pencil-lead break)

waveguide joint

Figure 3.10 Experimental setup for transmission efficiency determination of surface waveguides (Case E) 


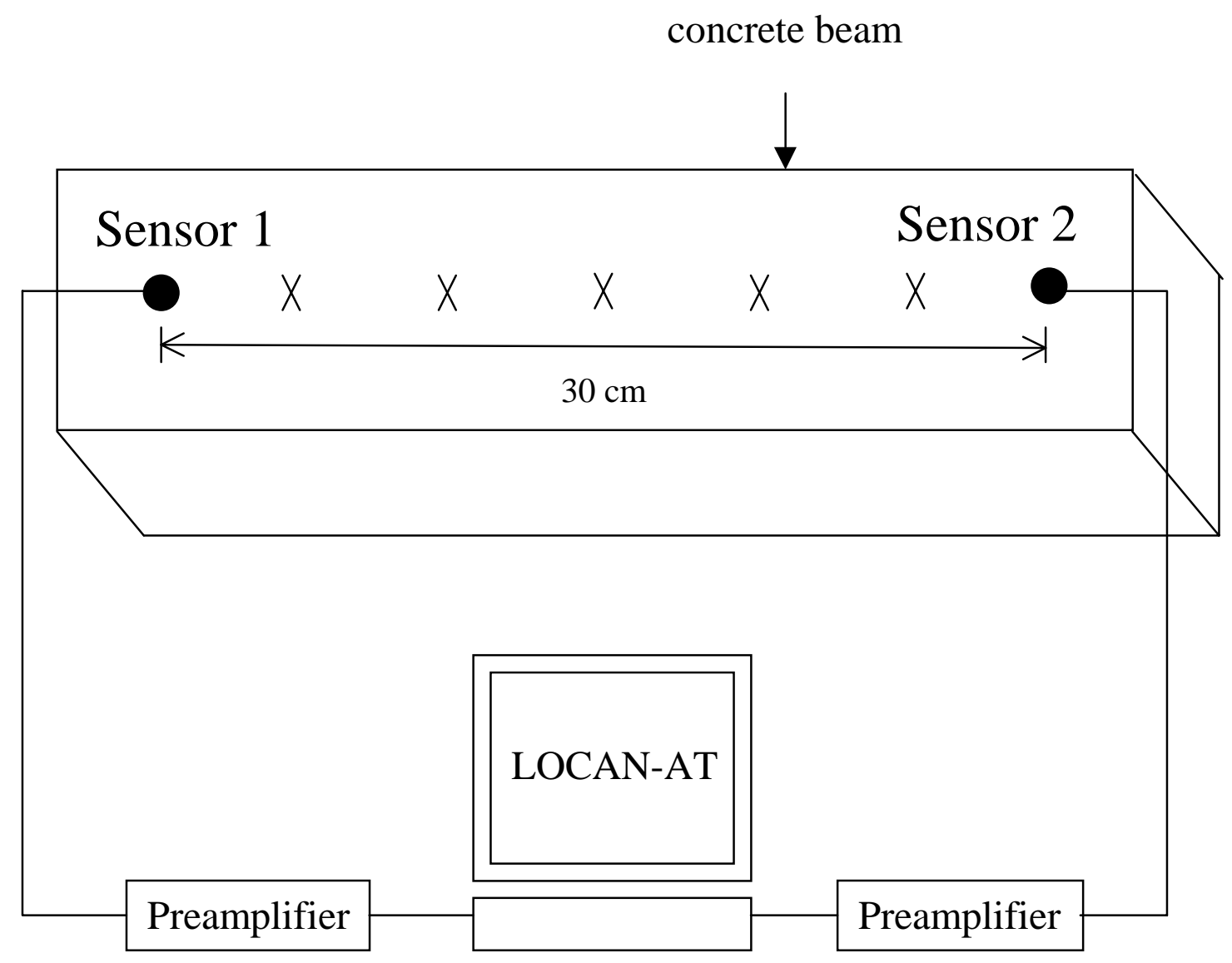

- PICO sensor

$X$ artificial AE source (pencil-lead break)

Figure 3.11 Experimental setup for attenuation measurement of concrete 


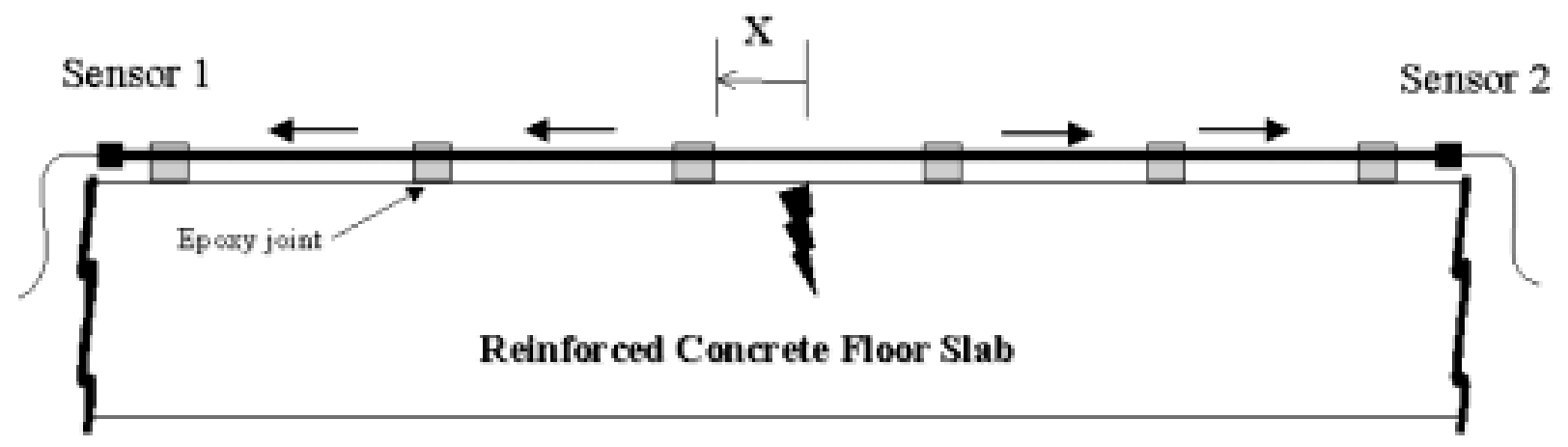

Figure 3.12 Propagation directions of AE signal in the surface waveguide 


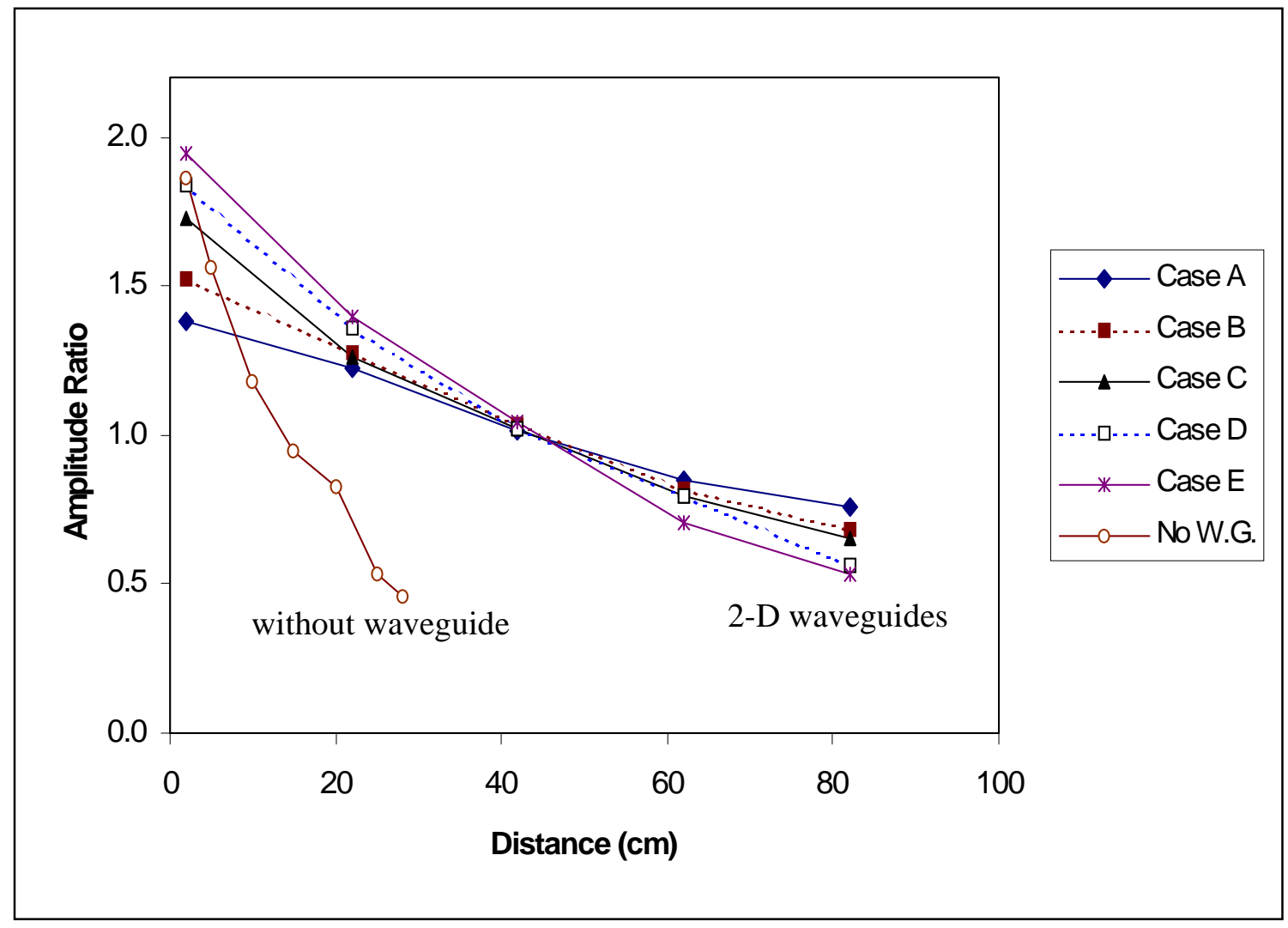

Figure 3.13 Attenuation curves of AE signals propagating in concrete and in surface waveguides 


\section{pencil-lead break}

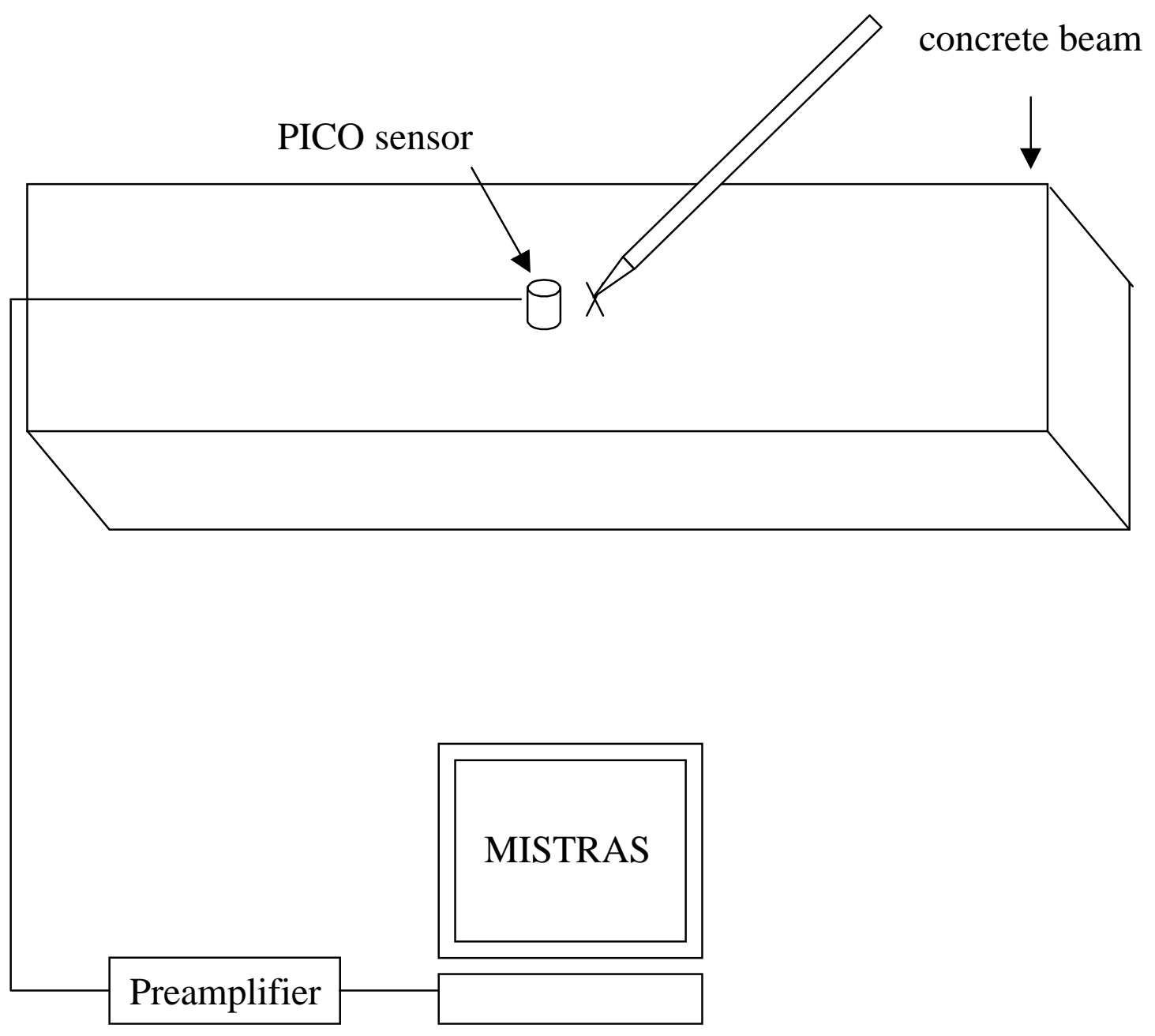

Figure 3.14 Experimental setup for the characteristic study of AE signals generated by standard pencil-lead breaks 


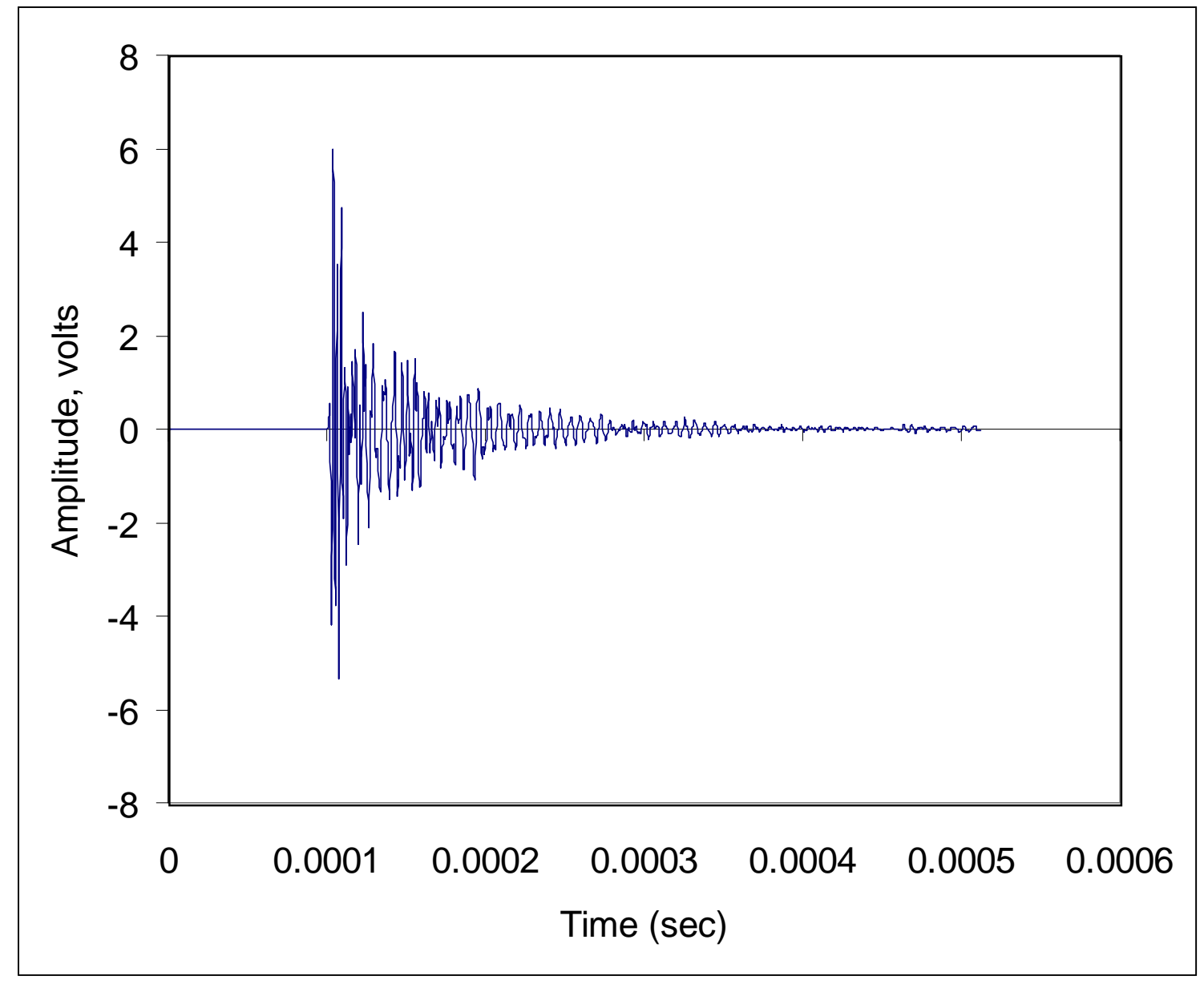

Figure 3.15 Typical AE signal generated by a pencil-lead break 


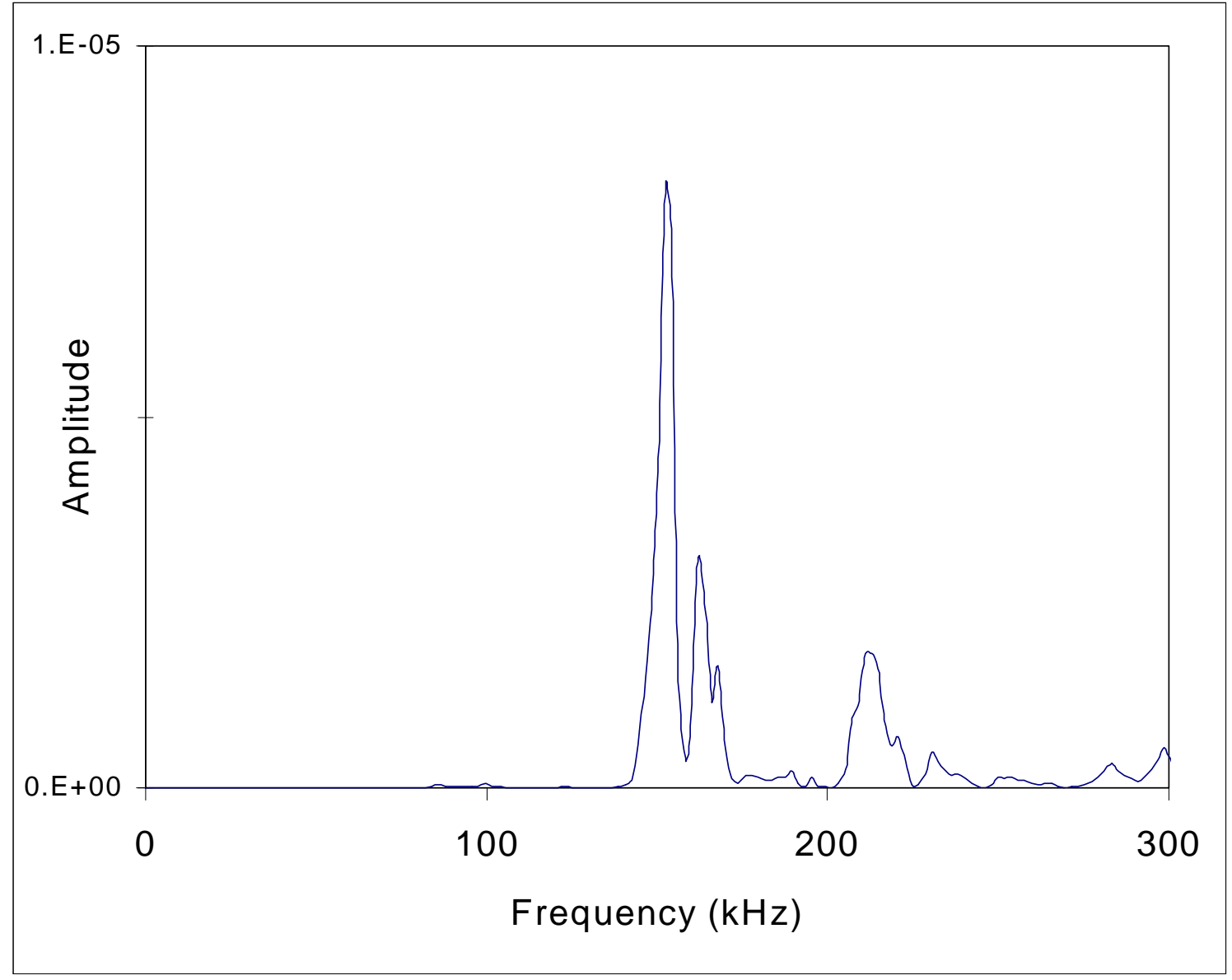

Figure 3.16 Frequency components of a typical AE signal generated by a pencil-lead break 


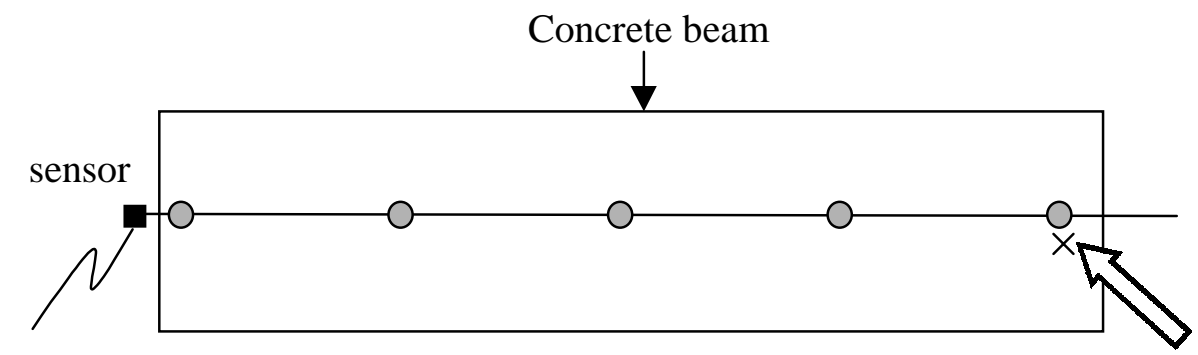

Pencil-lead break

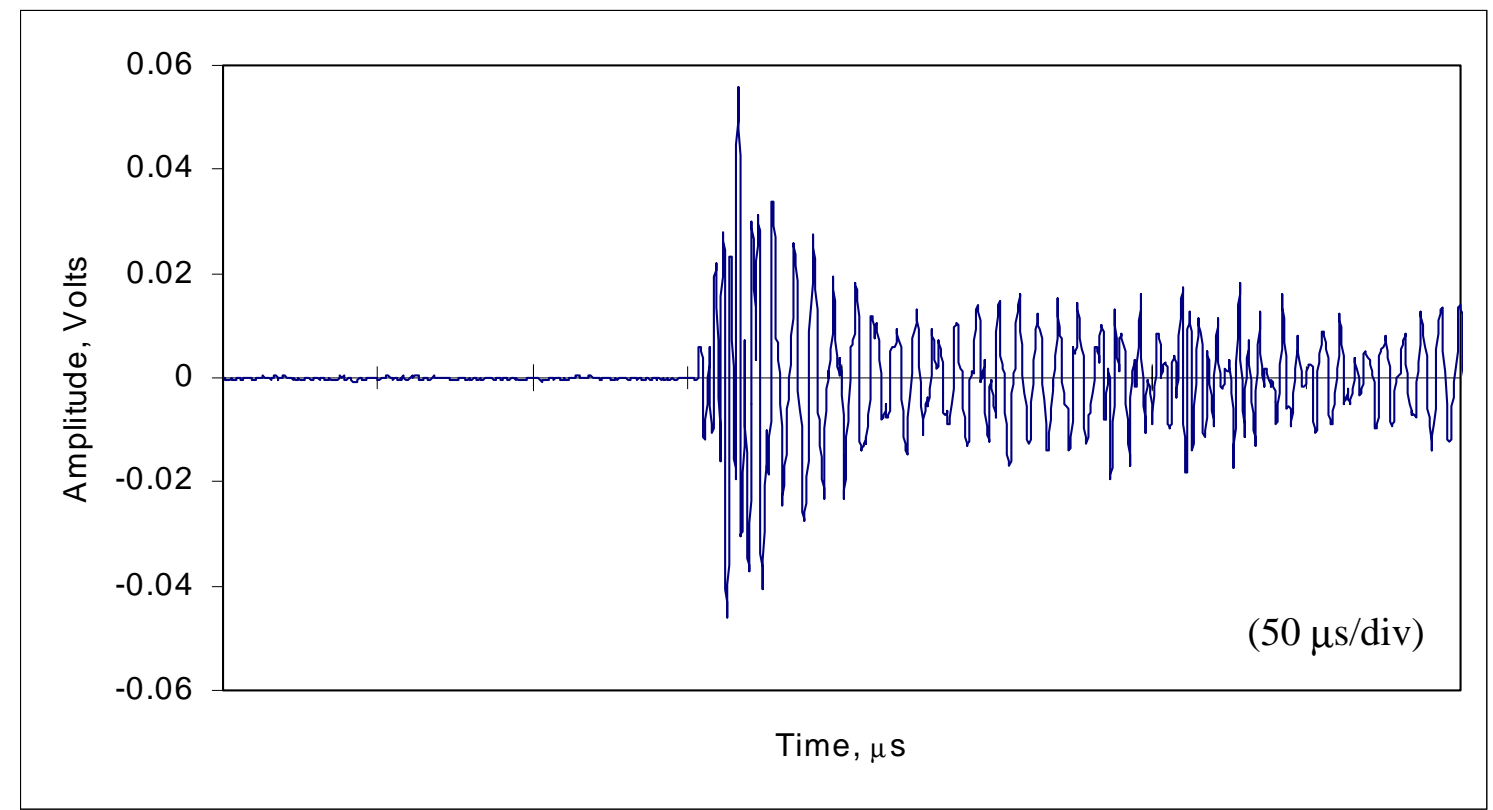

Figure 3.17 An example of an AE signal detected at the AE sensor after propagating through several epoxy joints 


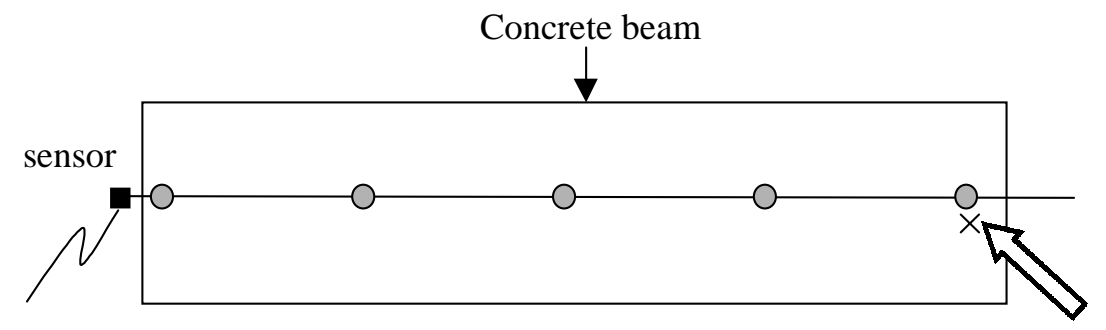

Pencil-lead break

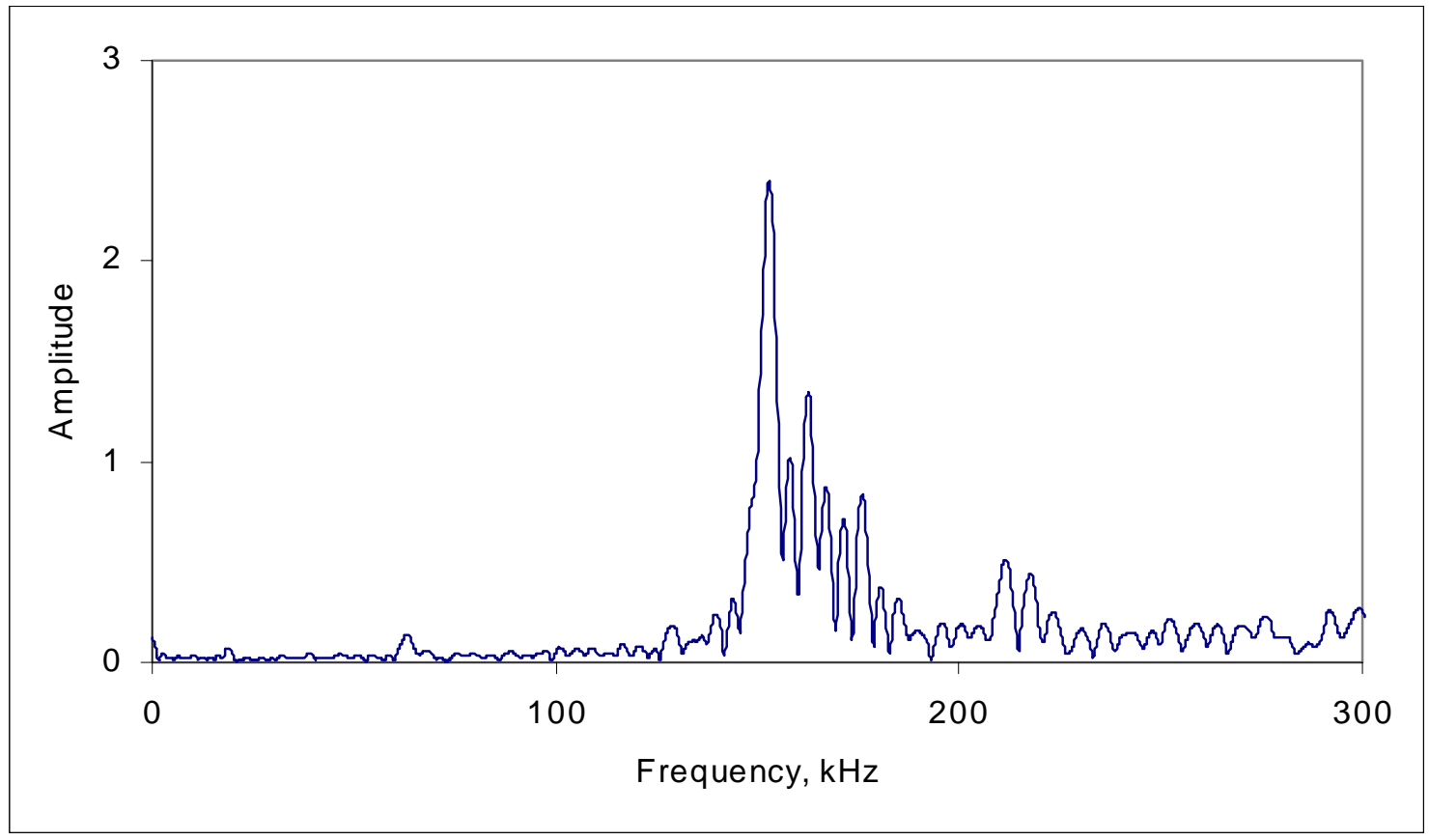

Figure 3.18 Frequency components of the AE signal detected at the AE sensor after propagating through several epoxy joints 


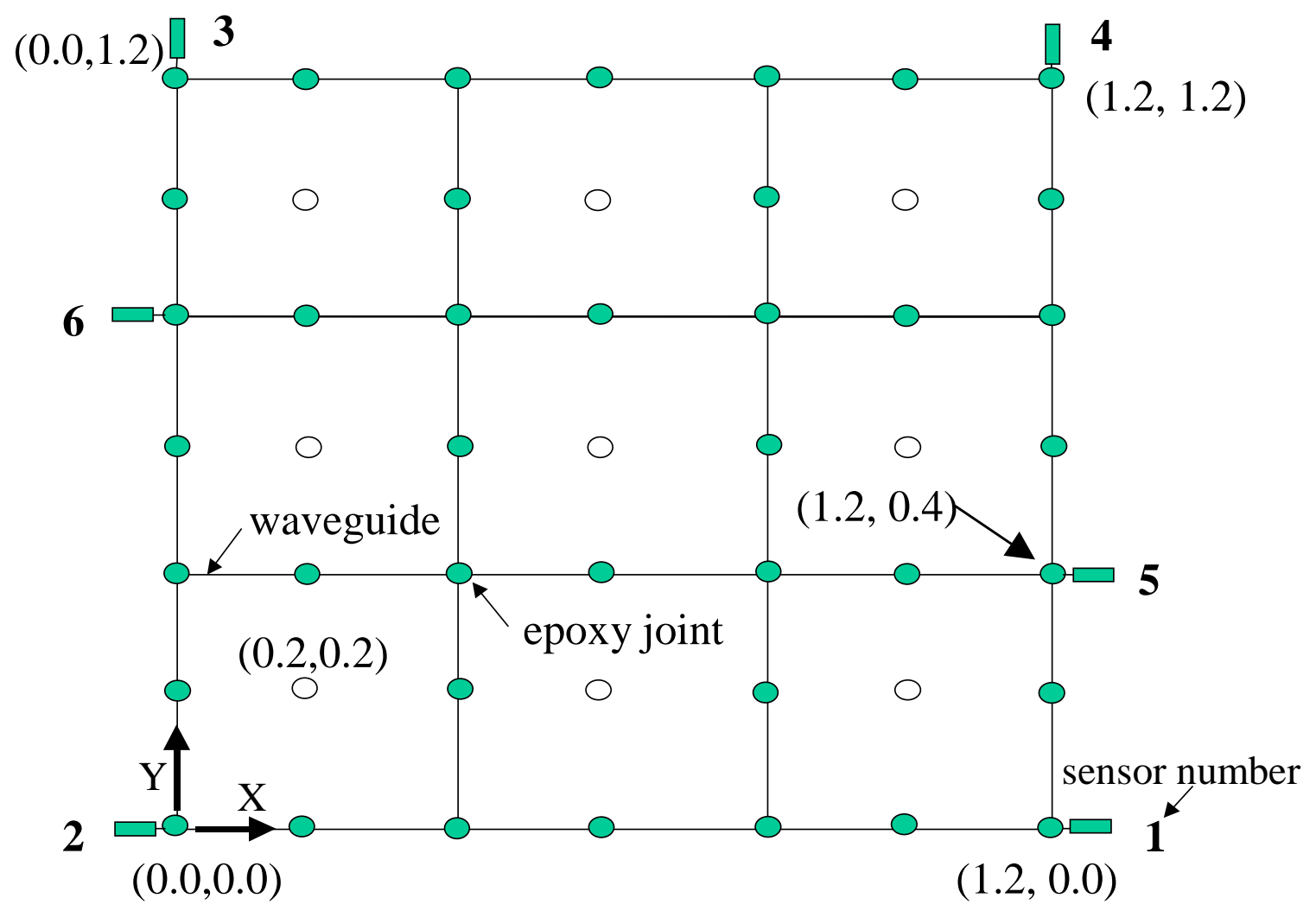

Figure 3.19 Coordinate system and pencil-lead break locations on the reinforced concrete floor slab 


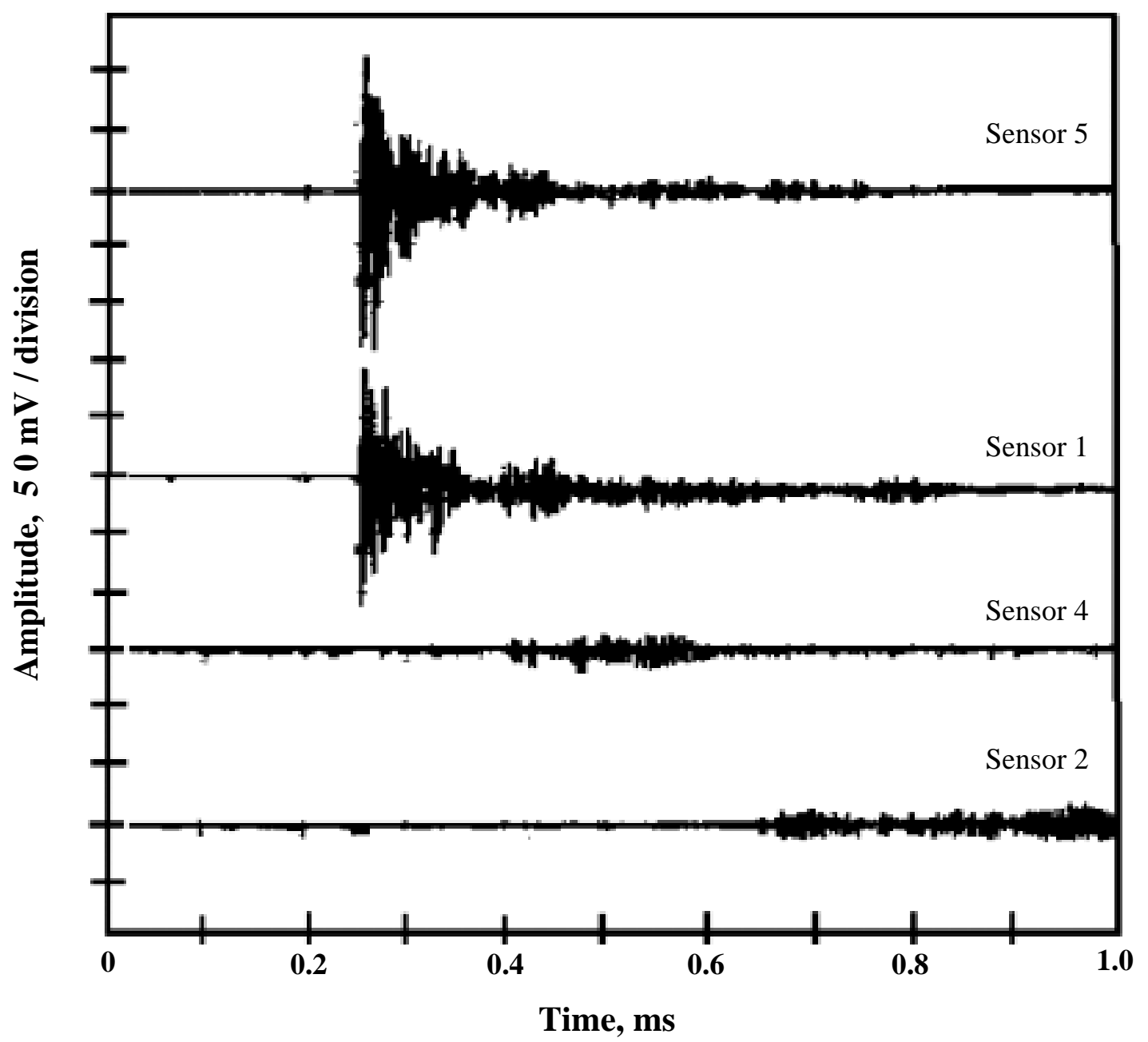

Figure 3.20 Detected AE signals due to a pencil-lead break at coordinate $(1.2,0.4)$ 


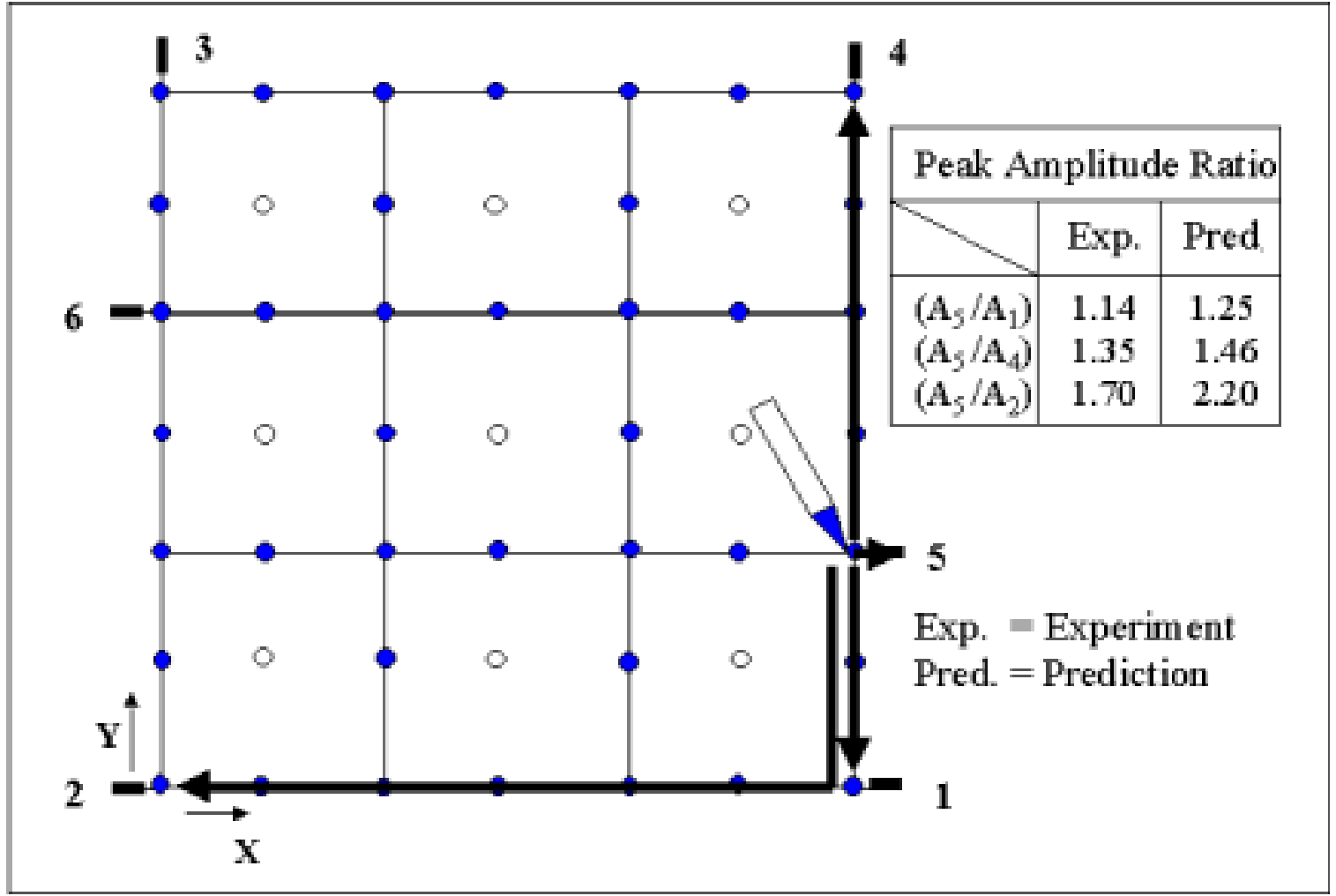

Figure 3.21 Predicted propagation routes due to the pencil-lead break at coordinate $(1.2,0,4)$ 


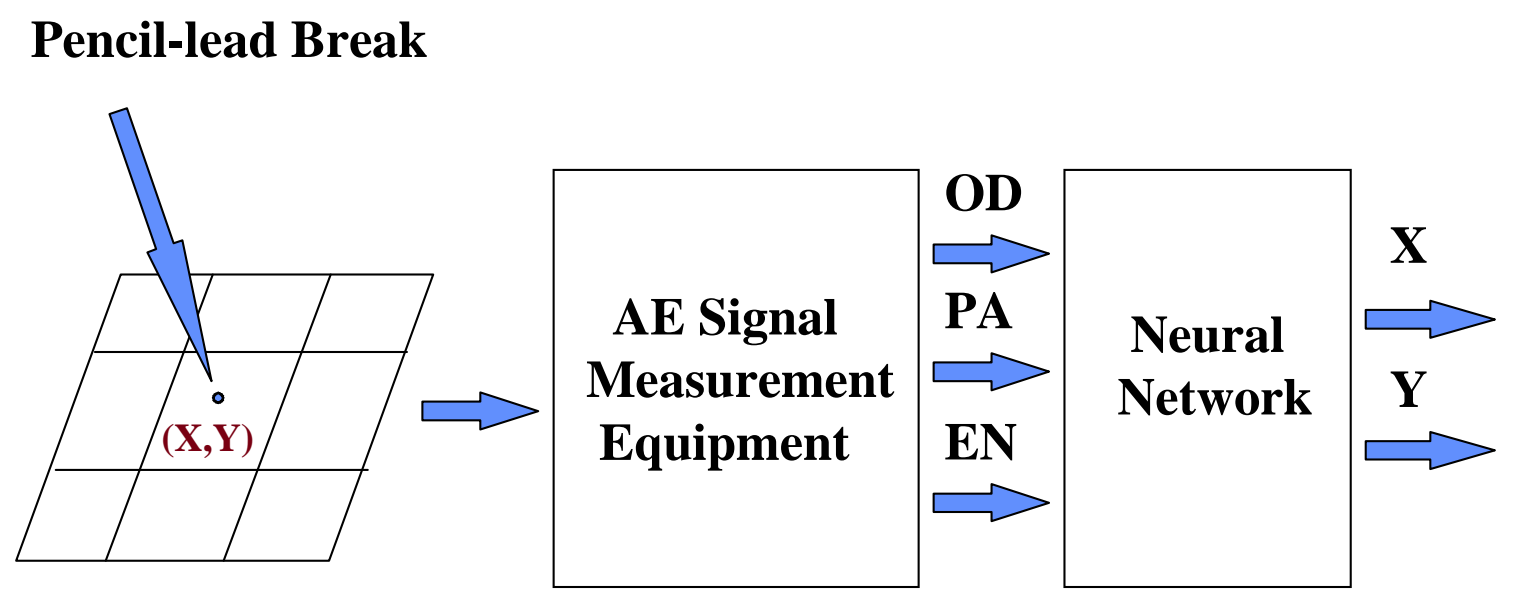

\section{OD : Order of Sensors \\ PA : Peak Amplitude \\ EN : Energy}

Figure 3.22 Schematic diagram of AE data processing system 


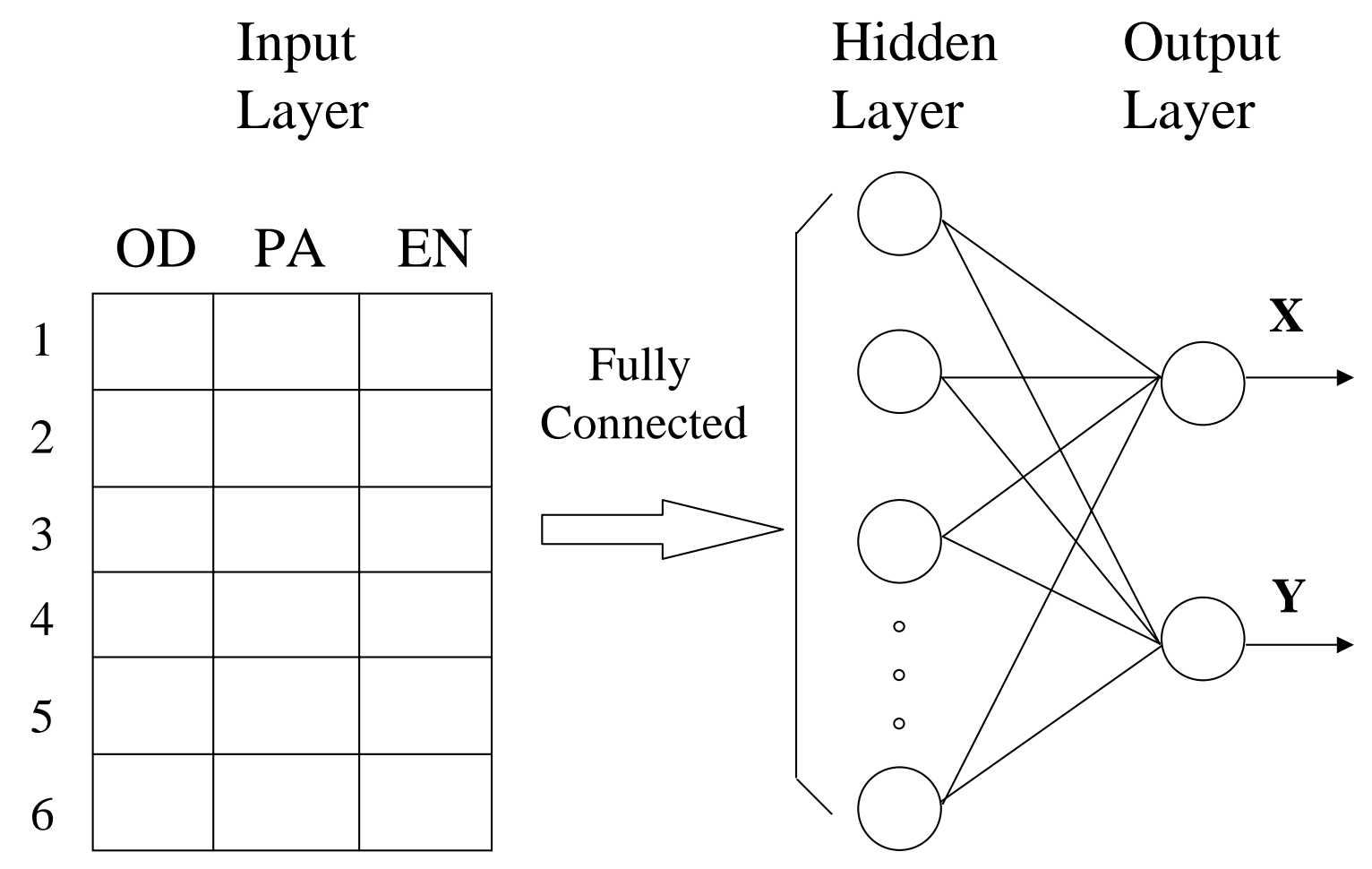

Figure 3.23 Architecture of the NN 


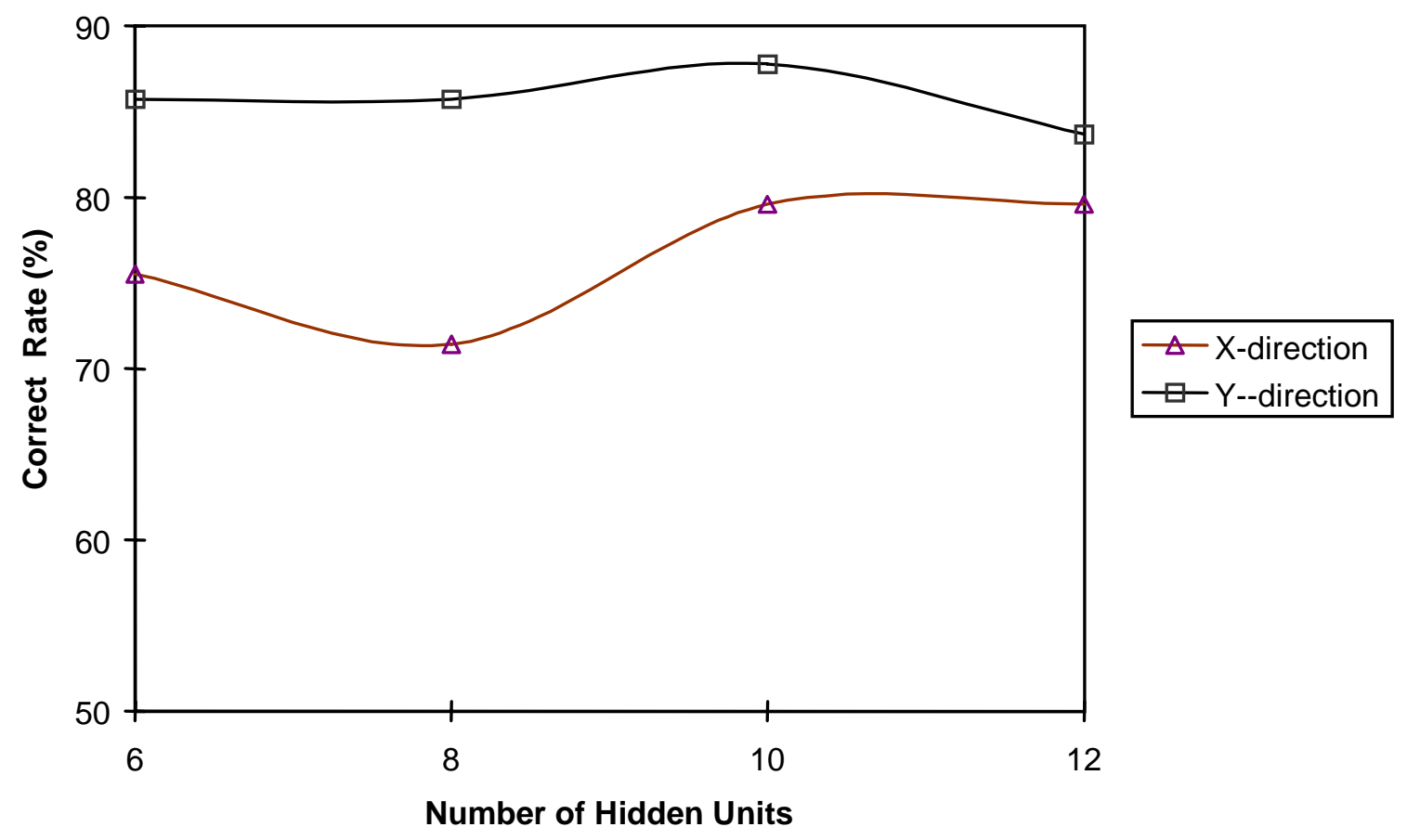

Figure 3.24 Correct rates of the testing data set versus number of hidden units of the NN 


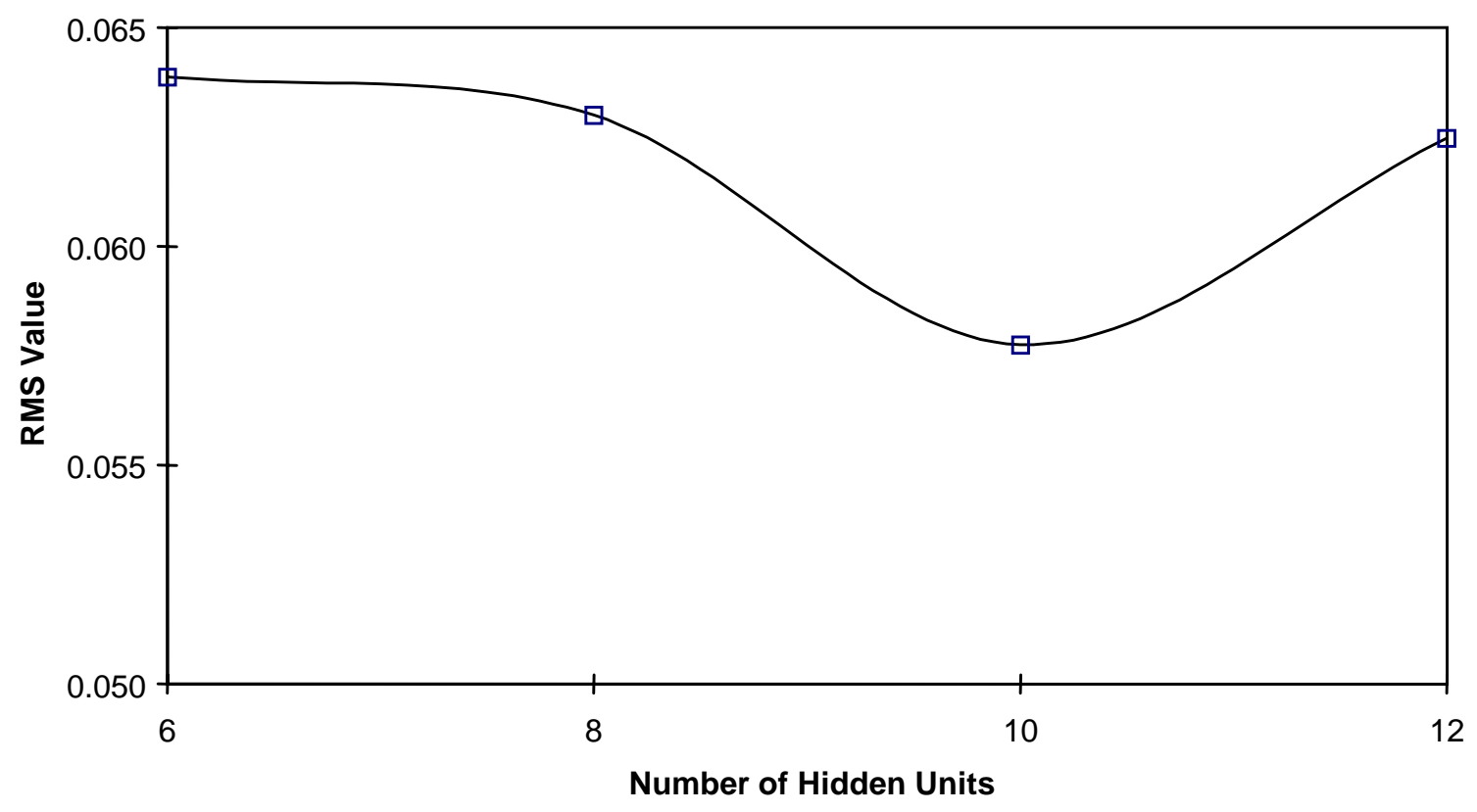

Figure 3.25 RMS value of the testing data set versus number of hidden units of the NN 


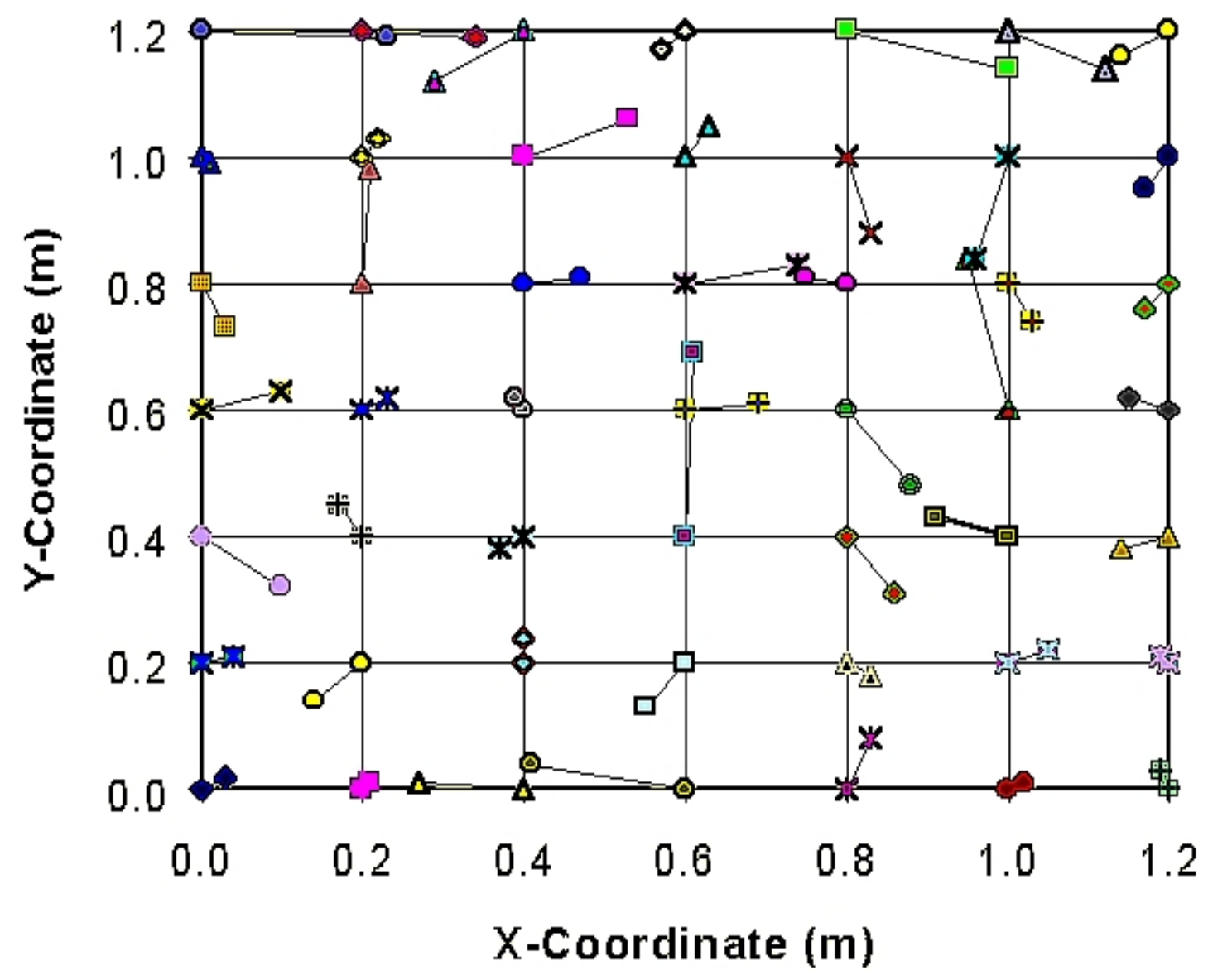

Figure 3.26 Graphical results of the NN predictions 


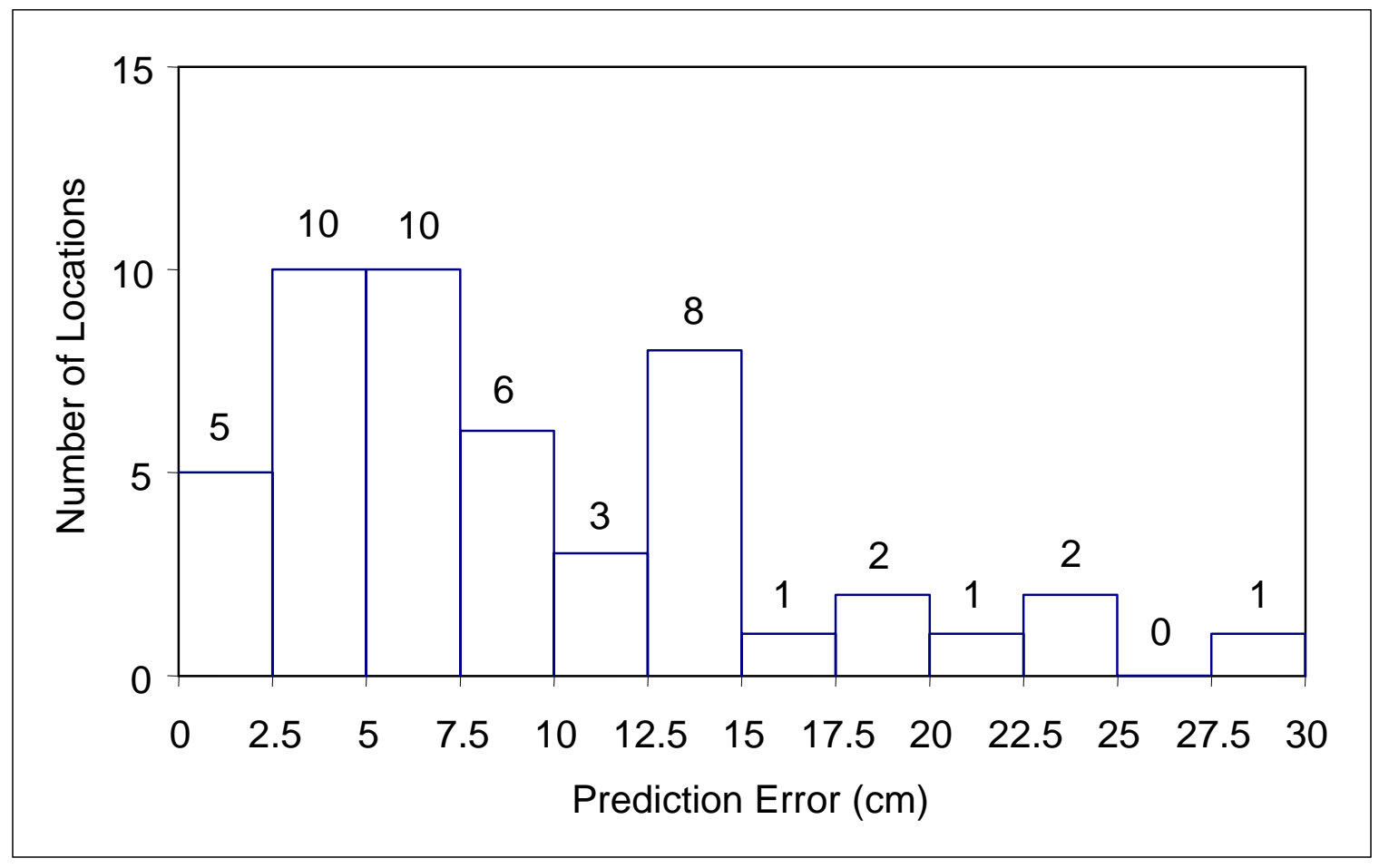

Figure 3.27 Prediction error of pencil-lead break locations from NN 


\section{CHAPTER 4}

\section{MEASUREMENT OF TENSILE FORCES IN SEVEN-WIRE PRESTRESSING STRANDS USING STRESS WAVES}

\subsection{OVERVIEW}

This chapter describes a laboratory experiment that was conducted in order to understand the actual behavior of stress waves propagating in a seven-wire strand that is subjected to different tensile forces. By taking into account of the acoustoelastic effect of an elastic solid and dispersive behavior of a stress wave, traveling time (or traveling speed) of each frequency component of the wave is not only dependent on the strand's material properties, but also dependent on tension level of the strand. Experimental part of this study is focused on measuring and comparing traveling times of longitudinal stress waves propagating along the length of a seven-wire prestressing strand subjected to different tensile forces. Theoretical studies and analyses are described in accordance with experimental results. Accuracy of the experimental measurements and analyses is also investigated in this chapter. 


\subsection{INSTRUMENTATION}

\section{Data acquisition system}

The MISTRAS 2001 (PAC), a fully digital, multichannel, computerized, acoustic emission system that performs AE signal and waveform measurements. Similar to the LOCAN-AT system, there are total of six monitoring channels in the MISTRAS system. Each channel requires its own sensor, preamplifier, and connecting cables. The advance feature of the MISTRAS system is its ability to record transient signals in time domain at a high speed data acquisition mode up to $8 \mathrm{MHz}$. Stress wave motions were first detected by an individual sensor and were acquired by each channel independently. Then, all of the data was transferred to the main processor of the MISTRAS system. Each individual recorded waveform can be displayed, analyzed, and exported.

\section{$\underline{\text { Sensors and Preamplifiers }}$}

Two PICO acoustic emission sensors (PAC) were used in this study. One was used as an input transducer and the other one was used as an output transducer. The sensor is 4.2 $\mathrm{mm}$ in diameter, which is about the same diameter size as the center wire of the seven-wire prestressing strand. The operating frequency range is between $100 \mathrm{kHz}$ and $1000 \mathrm{kHz}$.

One PAC-1220 preamplifier was used. It was connected to the output transducer. The preamplifier amplified and transfered AE signals to the MISTRAS system. The gain of $40 \mathrm{~dB}$ gain was used in this study. The preamplifier operates within a frequency range between $100 \mathrm{kHz}$ and $1200 \mathrm{kHz}$. 


\section{Pulse generator}

The C-101-HV (PAC) pulse generator was used in this study. The pulse generator provides an output pulse with an adjustable amplitude varying from 100 volts to 375 volts and a pulse width of $200 \mathrm{~ns}$. In this study, the highest amplitude level of 375 volts is used. Each individual output pulse is manually controlled by pushing a button on the rear panel of the pulse generator. In addition, the pulse generator also provides a trigger pulse on the rear panel which is synchronized with the output pulse.

\subsection{LABORATORY EXPERIMENTS}

A schematic drawing of the experimental setup is shown in Figure 4.1. A seven-wire stress-relieved strand of Grade 250 was used as a test specimen. The nominal diameter of the strand is $12.7 \mathrm{~mm}(1 / 2 \mathrm{inch})$. It is noted that grade designation for the strand corresponds to the minimum tensile strength in ksi unit. For the widely used seven-wire strands, two grades are available: Grade 250 and Grade 270. In this experiment, the strand is $4.76 \mathrm{~m}$ (15.62 ft) long and the actual length under tension is $4.46 \mathrm{~m}(14.63 \mathrm{ft})$. The strand is located inside a hollow core space throughout the entire length of a reinforced concrete beam. It was loaded by a hydraulic jack, and the tension levels were recorded by a load cell. Tensile force was gradually applied to the strand ranging from 0 kip to $25 \mathrm{kips}$. Two steel chucks were used to grip the strand at both ends where the tensile force was transferred.

At one end of the strand, an $\mathrm{AE}$ transducer and the pulse generator were used to generate a short duration impulse. At the other end of the strand, another AE transducer was 
used to capture the wave motions in the longitudinal direction. Both transducers were carefully mounted as perpendicular to the wire's cross section as possible to measure the longitudinal wave motions. The diameter of the AE transducer was about the same as the diameter of the center wire so that there was no contact between the transducers and the helical wires. A silicone grease compound, Ultragel II (Echo Ultrasound), was used as a coupling agent.

The typical test procedures were as follows. When the strand was stressed to a tension level, a stress wave was generated by the pulse generator, and at the same time a triggering signal was sent to the MISTRAS system as the reference time. The wave motion was detected by the output transducer. The detected wave motion was also recorded by the MISTRAS system. All signals were digitized at $8 \mathrm{MHz}$ sampling rate. Three data sets were recorded at each tension level. After each data set was recorded, the strand was unloaded, both transducers were checked to ensure a proper contact between the transducers and the wire, and the whole experimental setup was re-assembled prior to the recording of a new data set.

\subsection{TENSILE FORCE IN A CENTER WIRE}

Due to two different alignments between a center wire and helical wires in a prestressing strand, tensile forces between the two were not identical. Tensile forces in the center wire, $\mathrm{N}_{c}$, and each of the helical wires, $\mathrm{N}_{\mathrm{h}}$, can be illustrated by the following equation (Machida and Durelli, 1973): 


$$
\begin{gathered}
N_{c}=\frac{A_{c} N}{A_{c}+6 A_{h} \cos ^{3}(\beta)} \quad, \quad N_{h}=\frac{A_{h} \cos ^{2}(\beta) N}{A_{c}+6 A_{h} \cos ^{3}(\beta)} \\
N=N_{c}+6 N_{h} \cos (\beta)
\end{gathered}
$$

$\mathrm{N}$ is the axial tensile force applied to the strand. $\mathrm{A}_{\mathrm{c}}$ and $\mathrm{A}_{\mathrm{h}}$ are the cross-sectional areas of the center wire and the helical wires, respectively. $\beta$ is the lay angle of the helical wires $(\beta=$ $8^{\circ} 36^{\prime}$, Figure 4.2). Equations (4.1) and (4.2) give an estimated tensile force in the center wire, which is $14.7 \%$ of the applied load. In other words, tensile force in each of the helical wires is about $14.4 \%$ of the applied load. The actual tensile stress of the center wire $(\sigma)$ was then calculated and was carried on to the calculation of the longitudinal and transverse wave speeds. For practical purposes, the term "tensile force" in this study represents the tensile force applied to the entire seven-wire strand.

\subsection{CHANGE OF STRESS WAVE VELOCITIES DUE TO UNIAXIAL STRESS}

The change of velocities of the stress wave is due to the stress-induced anisotropy. This anisotropy is caused by the nonlinearities in the strain-displacement relationship and the constitutive relationship of the material. However, for simplicity, the center wire is considered as a slender, isotropic, elastic circular waveguide. The velocities of waves propagating along the waveguide, subjected to uniaxial tensile stress, are given (Murnaghan, 1951; Hughes and Kelly 1953; Johnson et al., 1986) as: 
Longitudinal wave velocity

$$
c_{L}^{2}=\frac{\lambda+2 \mu}{\rho}+\left(\frac{\lambda+2 l+(\mu+\lambda)(4 \lambda+10 \mu+4 m) / \mu}{3 \lambda+2 \mu}\right) \frac{\sigma}{\rho}
$$

$\underline{\text { Transverse wave velocity }}$

$$
c_{T}^{2}=\frac{\mu}{\rho}+\left(\frac{4 \lambda+4 \mu+m+\lambda n / 4 \mu}{3 \lambda+2 \mu}\right) \frac{\sigma}{\rho}
$$

where $\rho$ is the mass density of the steel strand and $\sigma$ is the tensile stress in a center wire. $\lambda$ and $\mu$ are Lame's (second-order) elastic constants, and $l, m$, and $n$ are Murnaghan's (thirdorder) elastic constants. It is worthwhile to mention that both $C_{L}$ and $C_{T}$ values are adversely proportional to the increase in tensile stress.

\subsection{WAVE PROPAGATION IN A CIRCULAR WAVEGUIDE}

The longitudinal wave propagating in a circular bar with traction free boundary conditions is considered. The Pochhammer's frequency equation (Archenbach, 1973), which governs the wave motion in a circular waveguide (or the center wire) with radius $a$ is given as: 


$$
\frac{2 p}{a}\left(q^{2}+k^{2}\right) J_{1}(p a) J_{1}(q a)-\left(q^{2}-k^{2}\right)^{2} J_{0}(p a) J_{1}(q a)-4 k^{2} p q J_{1}(p a) J_{0}(q a)=0
$$

where $\quad p^{2}=\frac{\omega^{2}}{C_{L}^{2}}-k^{2} \quad$ and $q^{2}=\frac{\omega^{2}}{C_{T}^{2}}-k^{2}$

$\omega$ is the angular frequency and $k$ is the wave number. $J_{0}(x)$ and $J_{l}(x)$ are the Bessel functions of the first kind of order 0 and 1 , and

$$
\begin{aligned}
& J_{0}(x)=\sum_{s=0}^{\infty} \frac{(-1)^{s} x^{2 s}}{2^{2 s}(s !)^{2}} \\
& J_{1}(x)=\sum_{s=0}^{\infty} \frac{(-1)^{s} x^{2 s+1}}{2^{2 s+1}(s !)(s+1) !}
\end{aligned}
$$

The frequency equation involves five independent quantities: $\omega, k, a, C_{L}$, and $C_{T}$. When the strand is subjected to a tensile force, the quantities $a, C_{L}$ and $C_{T}$ can be calculated. Derivations of the frequency equation are described in Appendix B. The group velocity $C_{g}$ at which the energy of wave motions propagates is defined as:

$$
C_{g}(\omega)=\frac{d \omega}{d k}
$$


From equations (4.5) and (4.8), it can be observed that wave motions in a slender rod are dispersive, in other words, the propagation velocity is a function of the frequency. The transient wave motion in a semi-infinite circular bar, due to a suddenly applied step force at its end, was reported by Folk et al. (1958). The wave motion at a particular time is usually composed of oscillations belonging to different frequency components. However, for a very long rod, since different frequencies of wave motions are propagating at different velocities, arrival time of a signal at any particular frequency can be calculated as

$$
t=\frac{z}{C_{g}(\omega)}
$$

where $C_{g}(\omega)$ is the group velocity of the wave motion at frequency $\omega$ and $z$ is the length of the rod (or the center wire in this case). Since the slenderness ratio of the center wire used in our experiment is greater than 1100 , the use of equation (4.9), which was derived from semiinfinite bar assumption, is reasonable. Thus, the arrival time $(t)$ of each frequency component of a propagating stress wave can be calculated.

\subsection{NUMERICAL ANALYSIS AND PREDICTION}

Numerical analysis was conducted based on the frequency equation [equation (4.5)], which can also be written in terms of dimensionless quantities as 


$$
\begin{aligned}
& {\left[2\left(\sqrt{\left(y / r^{2}\right)-x}\right)(y) J_{1}\left(\sqrt{\left(y / r^{2}\right)-x}\right) J_{1}(\sqrt{y-x})\right]-\left[(y-2 x)^{2} J_{0}\left(\sqrt{\left(y / r^{2}\right)-x}\right) J_{1}(\sqrt{y-x})\right]} \\
& -\left[4 x\left(\sqrt{\left(y / r^{2}\right)-x}\right)(\sqrt{y-x}) J_{1}\left(\sqrt{\left(y / r^{2}\right)-x}\right) J_{0}(\sqrt{y-x})\right]=0
\end{aligned}
$$

where $x=(k a)^{2}, y=\left(\frac{\omega \cdot a}{C_{T}}\right)^{2}$, and $r=\frac{C_{L}}{C_{T}} . \quad$ Thus, $k=\sqrt{\frac{x}{a^{2}}}$ and $\omega=\sqrt{\frac{y \cdot C_{T}^{2}}{a^{2}}}$.

The term $k a$ is called "dimensionless wave number" and the term $\left(\omega \cdot a / C_{T}\right)$ is called "dimensionless frequency". Consequently, the group velocity can be written as

$$
C_{g}(\omega)=\Delta\left(\frac{\sqrt{y}}{\sqrt{x}}\right) \cdot C_{T}
$$

By solving equation (4.10) for each $x$ value, multiple roots ( $y$ values) of this equation can be obtained. Each value of y belongs to each particular mode (first mode, second mode, third mode, and so on) in the longitudinal direction. In this study, only the smallest value of $y$, which belongs to the first longitudinal mode, is considered for the tensile force measurement. Using forward differentiation in equation (4.11), the group velocity of each frequency $(\omega)$ can be calculated.

Analytical results provide dispersion curves of a stress wave propagating in the center wire. Figure 4.3 shows an example of dispersion curves of a propagating stress wave when there was no tensile force applied to the strand. The diameter of the center wire is $4.2 \mathrm{~mm}$, 
and material properties of the wire used in the calculation are shown in Table 4.1. The dispersion curves describe the relationships between group velocity and frequency of the first two longitudinal modes. Considering the first mode, the group velocity is close to the bar velocity $C_{b}(5152 \mathrm{~m} / \mathrm{s})$ for a stress wave with a very low frequency component (point A). According to this velocity, the traveling time of such wave through the entire length of the strand $(4.76 \mathrm{~m})$ is $924 \mu \mathrm{s}$. For a stress wave with a very high frequency, the group velocity approaches the Rayleigh wave velocity $C_{R}(2944 \mathrm{~m} / \mathrm{s})$. In the range $\mathrm{AB}$, the group velocity decreases with increasing frequency. Hence, the waveform traveling with these frequency components would have the lower frequency components propagating faster (arriving earlier) than the higher frequency components. In the range after point $\mathrm{B}$, the group velocity increases with increasing frequency and will ultimately approach the velocity $C_{R}$. Note that the fastest group velocity of the second longitudinal mode is designated at point $\mathrm{C}$ (frequency $=1.2 \mathrm{MHz}$ and group velocity $=4300 \mathrm{~m} / \mathrm{s}$ ). At this speed, the second longitudinal mode would arrive at the receiving end in $1100 \mu \mathrm{s}$. Thus, the measurement of waveforms in the first longitudinal mode must be restricted within the time between $924 \mu$ s to $1100 \mu$ s to avoid the disturbance from the second longitudinal mode.

When the strand is subjected to a tensile force, time shift of the arriving waveforms would occur due to elongation of the strand. In addition, it was also shown from the numerical calculations (using equation 4.3 to equation 4.9) that group velocity of each single frequency component decreases when tensile force in the strand increases (Table 4.2). This is due to the reduction of the values from three parameters in the frequency equation: 1) the wire's diameter, $a$, 2) longitudinal wave speed, $C_{L}$, and 3) transverse wave speed, $C_{T}$. Thus, for the entire length of the strand, each particular frequency component travels with lower 
speed in the under-tension part of the strand and higher speed in the tension-free part (Figure 4.1). When the strand is subjected to different tensile forces, the velocities will change accordingly, and time shift of the waveform at any particular frequency component can be calculated.

\subsection{SIGNAL OBSERVATION}

Figure 4.4 shows a typical arrival waveform obtained from a laboratory test. The strand is subjected to 10 kips tensile force. The triggering signal from the pulse generator, as a reference point in time, is also illustrated in this figure. The early part of this waveform is enlarged and is shown in Figure 4.5. Cycle number is shown at the peak of each cycle. The first cycle of the waveform was identified as the first detectable cycle of the stress wave signal. The peak amplitude of this cycle is slightly higher than the average peak amplitude of the noise, which is about $1 \times 10^{-3}$ volts. By applying the Fourier transform technique, frequency components of the wavefront containing the first five and the first eighteen cycles are shown in Figure 4.6. Three data sets of the waveforms subjected to different tensile forces at 0 kips, 10kips, and 20 kips are illustrated. The result shows that frequency components are similar at all tension levels. The frequency components of the first five cycles (solid lines) constitute mainly from $150 \mathrm{kHz}$ to $350 \mathrm{kHz}$. The frequency range of the first eighteen cycles contains mainly the components from $150 \mathrm{kHz}$ to $400 \mathrm{kHz}$. This is well within the frequency range of the first longitudinal mode (Figure 4.3). The first eighteen cycles clearly show a greater contribution from the higher frequency components ranging from $250 \mathrm{kHz}$ to $400 \mathrm{kHz}$. This confirms that, for the first longitudinal mode that appeared 
at our monitoring range, the higher frequency components would arrive after the lower frequency components had arrived.

In order to verify the reproducibility of the wavefront, 3 sets of data were recorded at each tension level. After each data set was recorded, the strand was unloaded, both transducers were checked to ensure a proper contact between the transducers and the wire, and the whole experiment setup was re-assembled prior to the recording of the new data set. Figure 4.7, 4.8, and 4.9 show the comparison of the waveforms of three data sets recorded at different tension levels of 0 kip, 10 kips, and 20 kips, respectively. Results from these data sets show that the waveforms recorded at the same tension level have only minimal differences in time and amplitude. The slight deviations of the waveforms among different sets of data may be due to boundary condition changes at interfaces such as between the chucks and the strand, or between the transducers and the center wire. Nevertheless, at each tension level, the signals are clear and reproducible.

\subsection{PEAK IDENTIFICATION}

Figure 4.10 shows the early parts of the arrival waveforms due to two different tension levels. From this figure, the peaks of the waveforms have time shift when tension level of the strand increases from 10 kips to 15 kips. This time shift phenomenon is due to acoustoelasticity and dispersive behavior of the system. Using this figure as an example, time shift value in each of the first eighteen cycles is shown in Figure 4.11. It is noticed that there is only a slight variation in the time shift values among these cycles, which is about 1 data point deviation in our time measurement resolution $(0.125 \mu \mathrm{s})$. These early cycles of 
the waveform contain frequency components within a narrow range. The difference in group velocity among these cycles is still very small. Hence, the time shift is about constant at the first 18 cycles. Sensitivity of the time shift due to changes in propagation frequency will be discussed later in this chapter.

It is essential to correctly identify peaks of the same cycle between the two waveforms before measuring the time shifts. In order to do so, the first arrival cycle can be used as a starting point. However, because of its relatively small amplitude, locating the first cycle is sometimes difficult due to the influence from the background noise. Since every waveform has its own irregularities in shapes and amplitudes, there is a better way to identify the same cycle. For example, the wavefront shown in Figure 4.5 has its highest peak amplitude at the $11^{\text {th }}$ cycle. The peak amplitude reaches the lowest point at the $14^{\text {th }}$ cycle, and then rises up to reach another peak at the $16^{\text {th }}$ cycle. From this amplitude shape, it is now possible to compare the peaks between two waveforms started from the latter (referenced) cycles with irregularities. Figure 4.12 illustrates the time shifts of waveforms due to increasing tension levels from 10 kips to 25 kips. It is observed that the recorded waveforms shift forward when the tensile force increases. It is also observed that there are small changes in shapes and amplitudes of the detected waveforms when higher tensile forces were applied to the strand. This is due to the dispersion of the stress wave propagating over a longer strand length, which was subjected to higher tensile forces. This dispersion of the stress wave might cause some difficulties in identifying the same cycle number of different waveforms (Figure 4.12). However, matching same cycle number can be conducted precisely by observing and comparing several irregularities of the shape of the waveforms over a large number of cycles (e.g. first 40 cycles) so that correct time shift 
measurement can be ensured. Another example of matching the same cycle is illustrated in Figure 4.13 where two waveforms due to 24 kips and 25 kips tensile forces are compared. In this figure, many irregularities are shown (e.g. at the $23^{\text {th }}$ cycle) and the same cycle numbers between the two waveforms can be matched.

\subsection{TIME SHIFT OF WAVEFORMS DUE TO DIFFERENT TENSILE FORCES}

Figure 4.14 shows the calculated and the measured peak arrival times of the first five cycles of the wavefronts at different tension levels. The dotted lines represent the calculated arrival times of stress waves propagating in the first longitudinal mode. For each dotted line, the numerical results were obtained based on a single frequency assumption. It is shown that peak arrival times of the first cycle have good agreement with the arrival times of a stress wave propagating with a single frequency component of $150 \mathrm{kHz}$. As previously described (Folk et al., 1958), a particular time on the waveform can be approximated using a single frequency component. Figure 4.14 shows that the measured peaks from the second cycle at different tension levels have the same frequency component of about $185 \mathrm{kHz}$. The approximated frequency values of the latter peaks from the third cycle to the fifth cycle are also shown in this figure. The results from the Fourier transform technique (Figure 4.6) also confirm that the early cycles of the waveforms contain dominant frequency components from $150 \mathrm{kHz}$ to $250 \mathrm{kHz}$ (the first 5 cycles). These frequency components correspond well with the calculated frequency shown in Figure 4.14. This result shows that the single frequency approximation has good agreement with the experimental data at each tension level. 
After a single frequency representing the peak of one particular cycle was selected, time shifts of the waveforms were calculated to compare with the time shifts from the experiment (Figure 4.15). Our numerical results show that there is only a very slight nonlinearity between the time shift and the tension level. This is also verified by the experimental observation. Time shifts obtained from the numerical analysis and from the experimental measurements have good agreement at each tension level as shown in Figure 4.15.

\subsection{DISCUSSIONS}

Experimental results (Figure 4.13) show that there is a slight nonlinearity of the time shift under high tension levels from 23 kips to 25 kips. The time shift values of those three data points at high tension levels are slightly off the calculated values (solid line). This is possibly due to the nonlinear behavior of the steel when it is subjected to a high tensile stress level. At $25 \mathrm{kips}$ tension level, the stress level is $174 \mathrm{ksi}$ and is about $70 \%$ of the ultimate strength of the strand.

It was also observed that the time shifts of the waveforms at tension level below 10 kips, were not consistent. When the tensile force was increased, the helical wires tend to straighten more as the strand was loaded, and the gripping force applied from the chucks was relatively small so that slippage between the center and the helical wire might occur. Thus, the tensile force in the center wire was released from time to time, causing inconsistent time shifts of the waveforms. This was observed when tensile force was applied to the strand up to about $18 \%$ of its ultimate strength. Hence, the current evaluation technique has been 
demonstrated to evaluate the prestress force only at the range between $18 \%$ to $70 \%$ of the ultimate strength of the Grade 250 seven-wire strand.

Table 4.3, as an example, shows a comparison between the calculated time shift of the predicted frequency of the first cycle $(150 \mathrm{kHz})$ and the calculated time shift of several other frequencies ranging from $145 \mathrm{kHz}$ to $155 \mathrm{kHz}$. As shown in this table, there are very small differences among the calculated time shift within this narrow frequency band. This is also the case for the calculated time shift of the latter cycles. It is shown that the time shift is not strongly affected by a small variation in the single frequency approximation (e.g. $\pm 5 \mathrm{kHz}$ ) for each cycle.

As seen in Figure 4.15, the estimation of a tensile force at any particular time shift value has an error less than 1 kip or about $6.9 \mathrm{ksi}$, which is approximately $2.76 \%$ of the ultimate strength of the strand $(250 \mathrm{ksi})$. The sampling rate of $8 \mathrm{MHz}$, providing a time measurement resolution of $0.125 \mu \mathrm{s}$, is sufficient for recording the arrival parts of the waveforms, which have frequency components ranging from $150 \mathrm{kHz}$ to $350 \mathrm{kHz}$. Operating frequency range and sensitivity of the transducers were carefully selected so that clear and strong signals at the receiving end of the strand were detected. The accuracy of this technique would be affected by several factors such as coating of strand, temperature at the time of measurement, and type of strands used. Investigation of these factors should be conducted in the future. 


\subsection{SUMMARY}

The experimental and analytical results presented indicate that the traveling time of the stress wave propagating inside the center wire of a seven-wire prestressing strand can be related to the stress level of the strand. Changes in the traveling time are due to the elongation of the strand together with the changes in the stress wave velocities. In this chapter, a numerical calculation based on the frequency equation is conducted by considering the elastic waveguide theory and the changes of wave speeds at different stress levels. The proposed evaluation technique was shown to work well when the prestress force ranges between $18 \%$ and $70 \%$ of the ultimate strength of the strand. This technique can be used as an efficient tool for measuring tensile forces in the post-tensioning strands of a prestressed concrete structure. 


\begin{tabular}{|c|cc|}
\hline & GRADE 250 \\
\hline$\rho$ & $=7.300 \times 10^{-4} \quad\left(\mathrm{lb}-\mathrm{s}^{2} / \mathrm{in}^{4}\right)$ \\
\hline$\lambda$ & $=16.79 \times 10^{6}$ & $(\mathrm{psi})$ \\
\hline$\mu$ & $=11.59 \times 10^{6}$ & $(\mathrm{psi})$ \\
\hline 1 & $=-35.960 \times 10^{6}$ & $(\mathrm{psi})$ \\
\hline $\mathrm{m}$ & $=-90.34 \times 10^{6}$ & $(\mathrm{psi})$ \\
\hline $\mathrm{n}$ & $=-103.5 \times 10^{6}$ & $(\mathrm{psi})$ \\
\hline
\end{tabular}

Where $\rho$ is the mass density; $\lambda$ and $\mu$ are Lame's elastic constants. $1, \mathrm{~m}$, and $\mathrm{n}$ are Murnaghan's elastic constants.

The nominal cross-sectional area of the strand is $0.144 \mathrm{in}^{2}$.

Table 4.1 Calculated material constants of the seven-wire prestressing strand (Grade 250). 


\begin{tabular}{|c|c|c|c|c|c|c|}
\hline \multirow{2}{*}{$\begin{array}{c}\text { Strand } \\
\text { Tensile } \\
\text { Force } \\
\text { (kips) }\end{array}$} & \multirow{2}{*}{$\begin{array}{c}\text { Tensile } \\
\text { Stress } \\
(\mathrm{ksi}) \\
\end{array}$} & \multicolumn{5}{|c|}{ Group Velocity $(\mathrm{m} / \mathrm{s})$} \\
\hline & & Bar velocity & $150 \mathrm{kHz}$ & $200 \mathrm{kHz}$ & $250 \mathrm{kHz}$ & $300 \mathrm{kHz}$ \\
\hline 0 & 0 & 5152 & 5100 & 5056 & 4994 & 4909 \\
\hline 10 & 69.4 & 5143 & 5092 & 5048 & 4987 & 4904 \\
\hline 20 & 138.9 & 5133 & 5083 & 5041 & 4981 & 4899 \\
\hline
\end{tabular}

Table 4.2 Change of group velocity due to tensile stresses in the center wire $(\varnothing=4.2 \mathrm{~mm})$.

\begin{tabular}{|c|c|c|c|c|}
\hline \multirow{2}{*}{ Frequency } & \multicolumn{2}{|c|}{ Arrival Time $(\mu \mathrm{s})$} & \multirow{2}{*}{$\begin{array}{c}\text { Time } \\
\text { Shift } \\
(\mu \mathrm{s})\end{array}$} & $\begin{array}{c}\text { Time Shift } \\
\text { Difference } \\
(\%)\end{array}$ \\
\cline { 2 - 3 } & Tensile Force = 0 kips & Tensile Force =25 kips & 9.12 & $+0.22 \%$ \\
145 & 933.20 & 942.32 & 9.11 & $+0.11 \%$ \\
148 & 933.59 & 942.70 & 9.10 & $0.00 \%$ \\
150 & 933.86 & 942.96 & 9.09 & $-0.11 \%$ \\
152 & 934.13 & 943.22 & 9.07 & $-0.33 \%$ \\
155 & 934.54 & 943.62 & & \\
\hline
\end{tabular}

Table 4.3 Calculated time shift of different frequency components. 


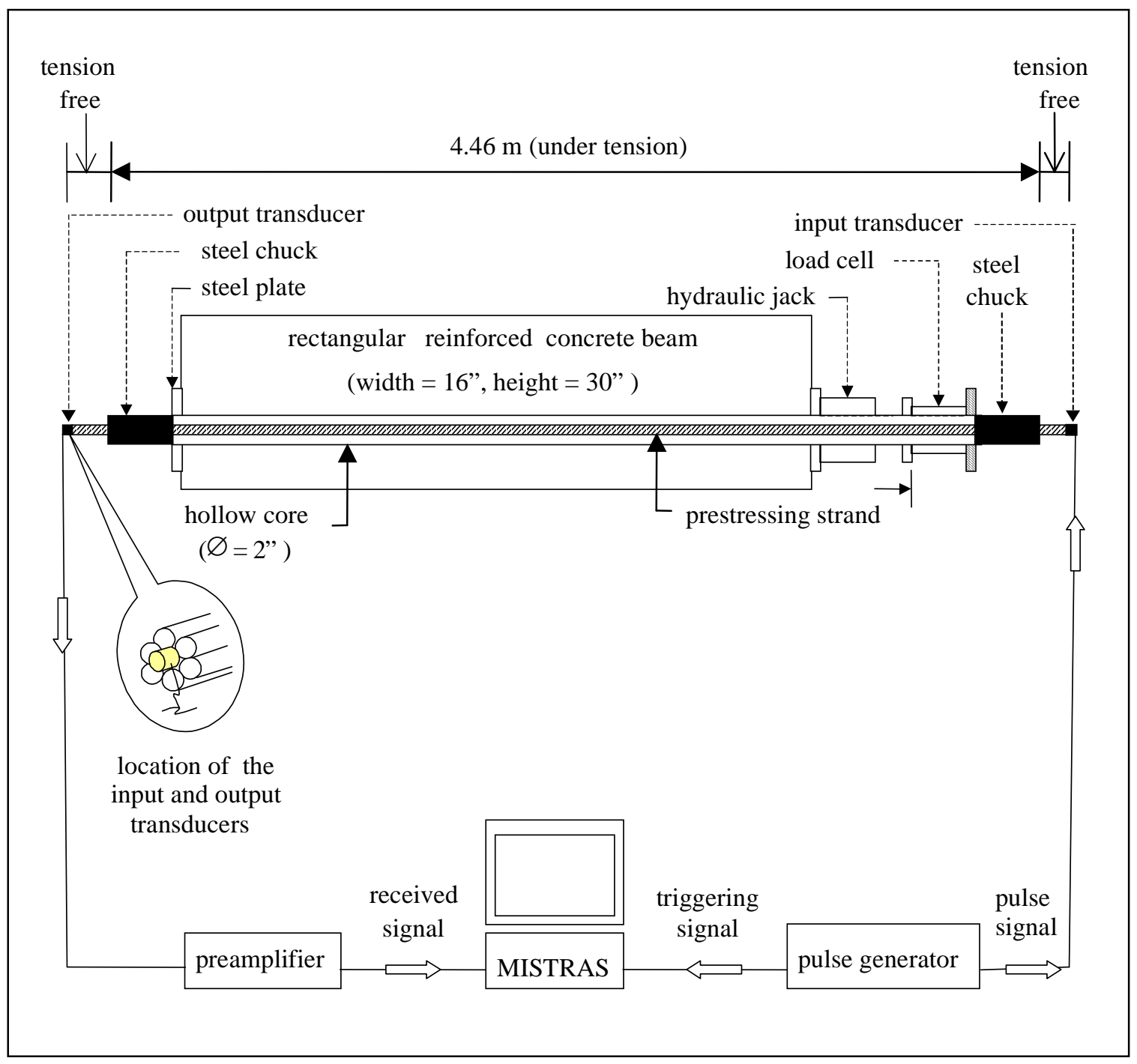

Figure 4.1 Experimental setup. 


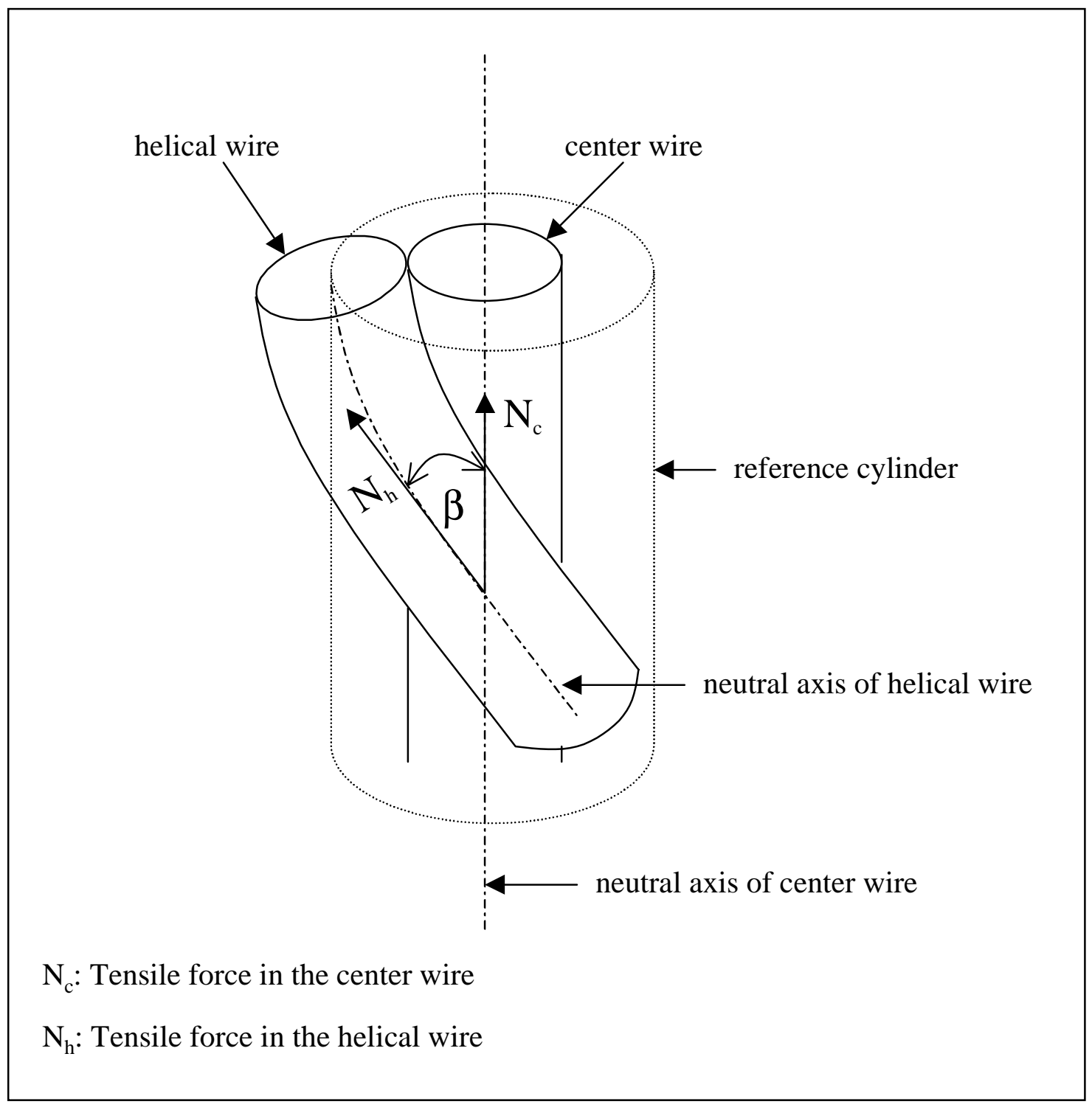

Figure 4.2 Lay angle of a helical wire $(\beta)$ 


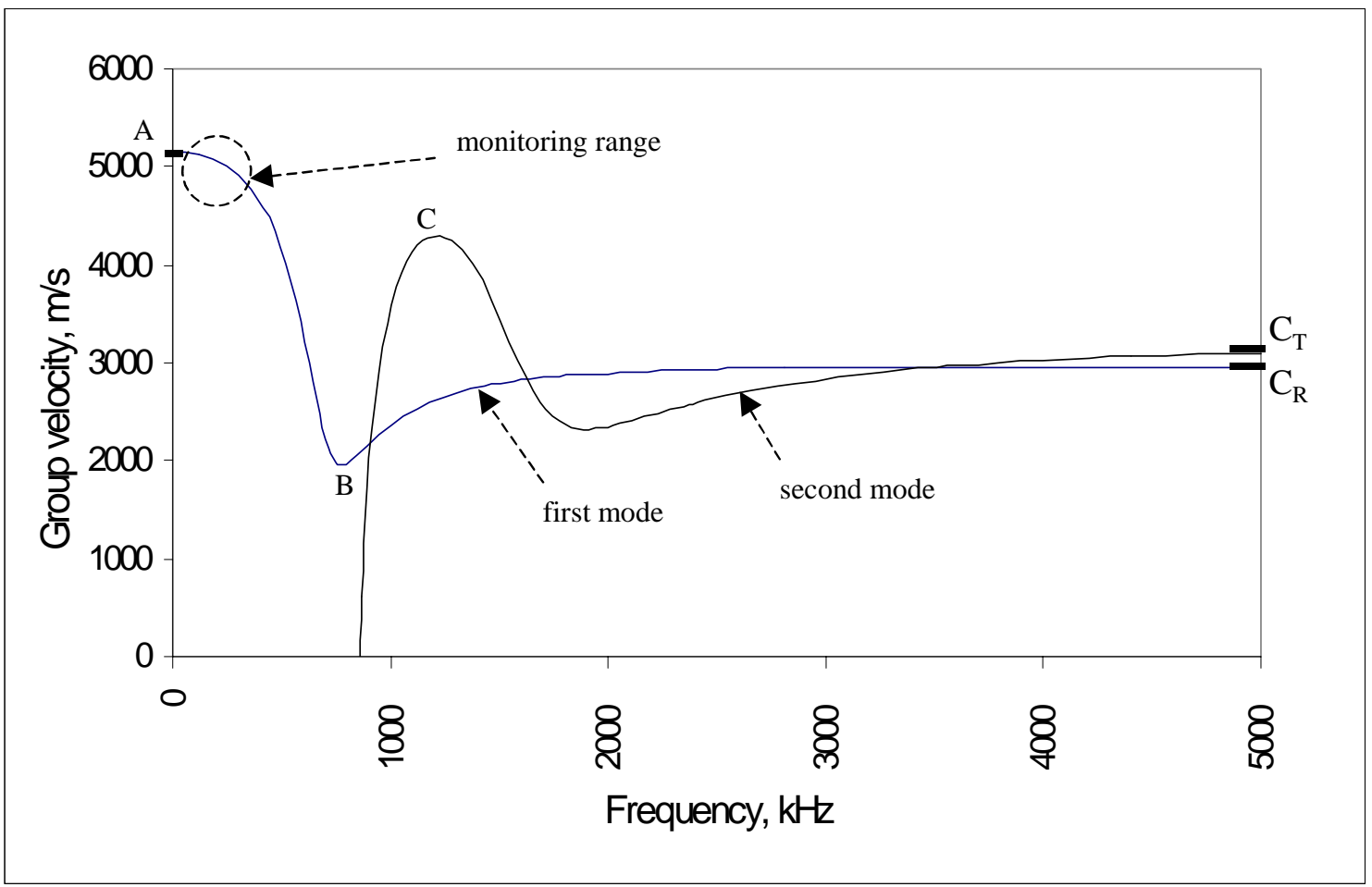

Figure 4.3 Group velocity of the first two longitudinal modes for the center wire $(\varnothing=4.2 \mathrm{~mm})$. There is no tension applied. 


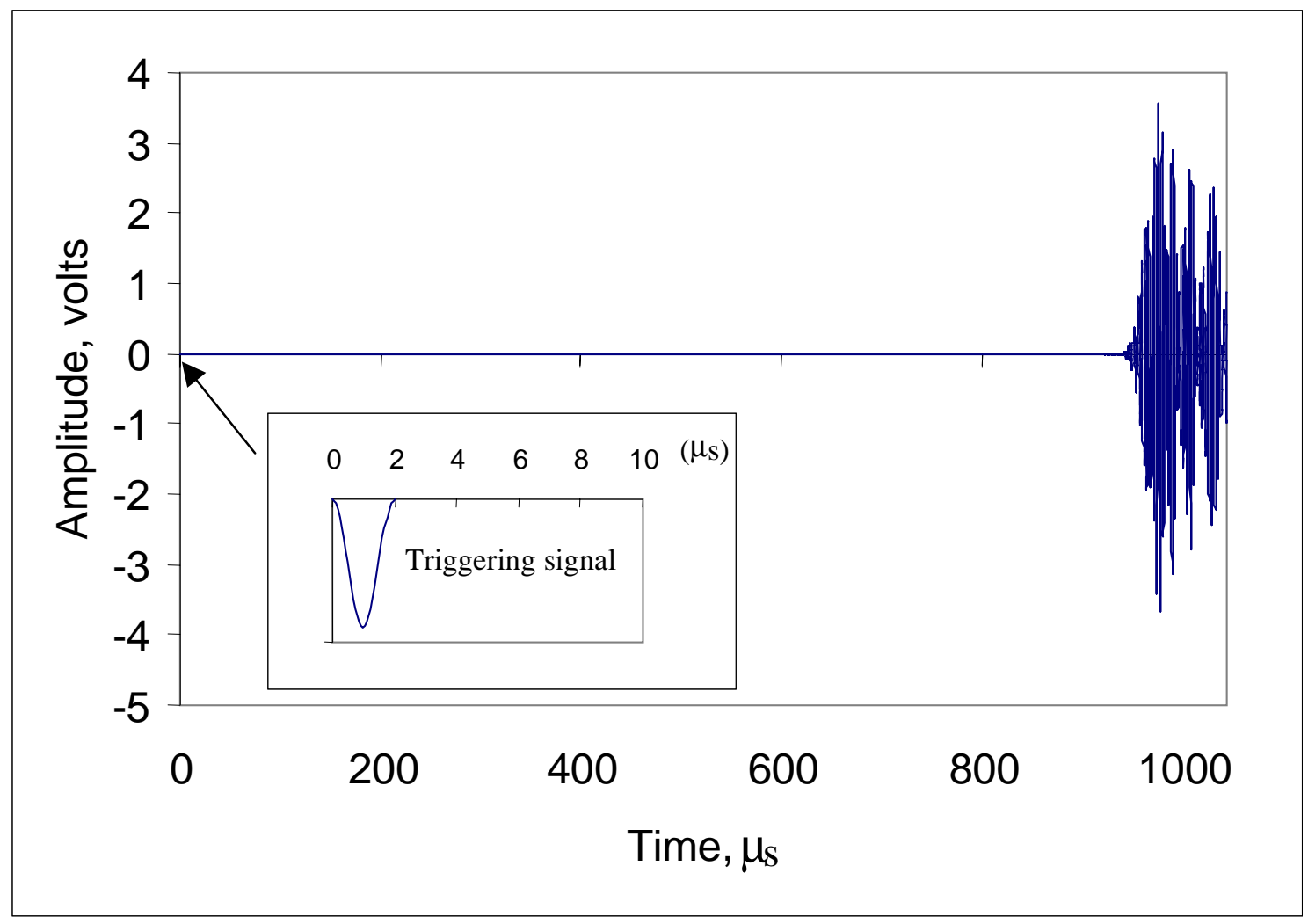

Figure 4.4 Waveform recorded at receiving end of the strand subjected to $10 \mathrm{kips}$ tensile force. 


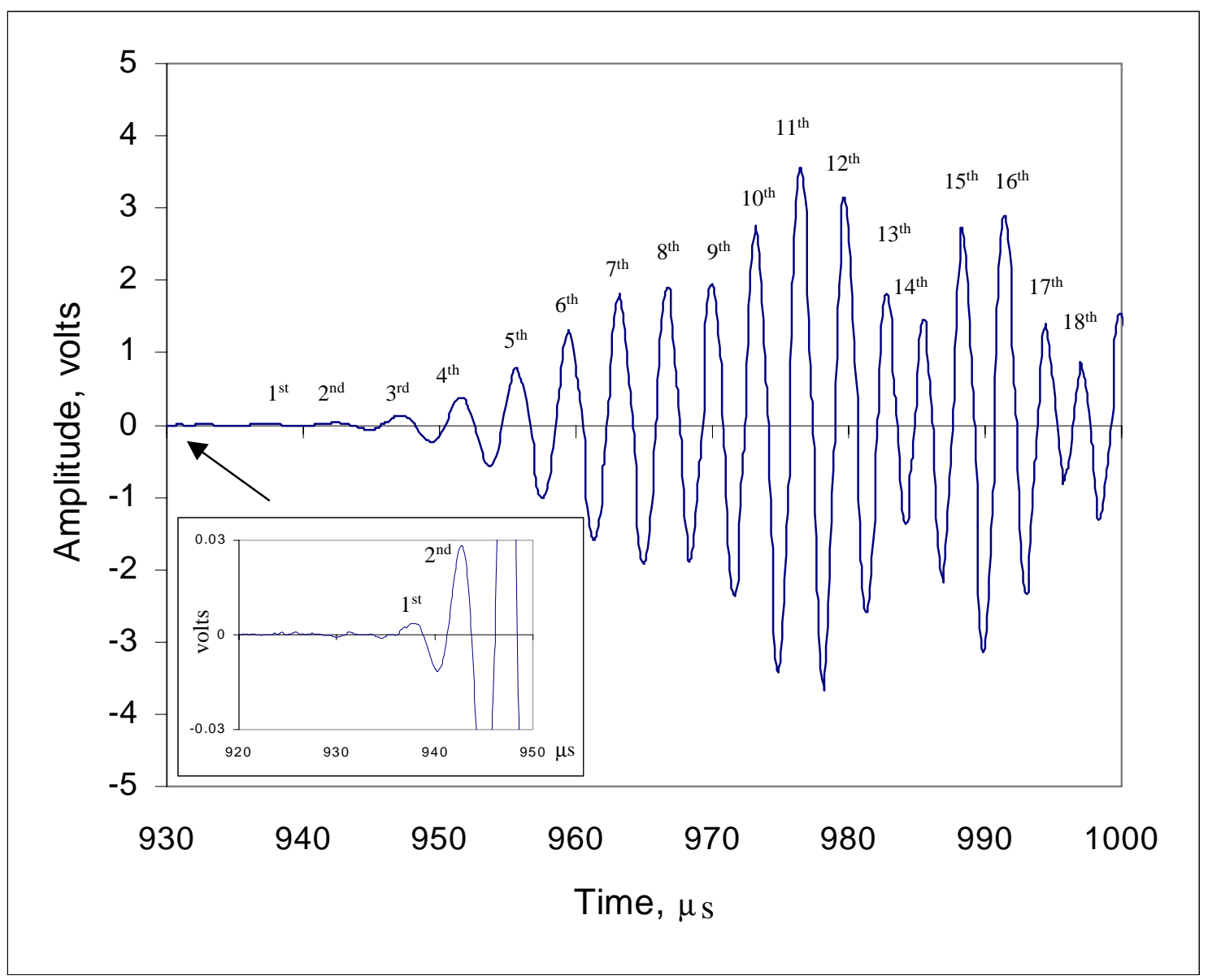

Figure 4.5 Early part of the waveform due to 10 kips tensile force. 


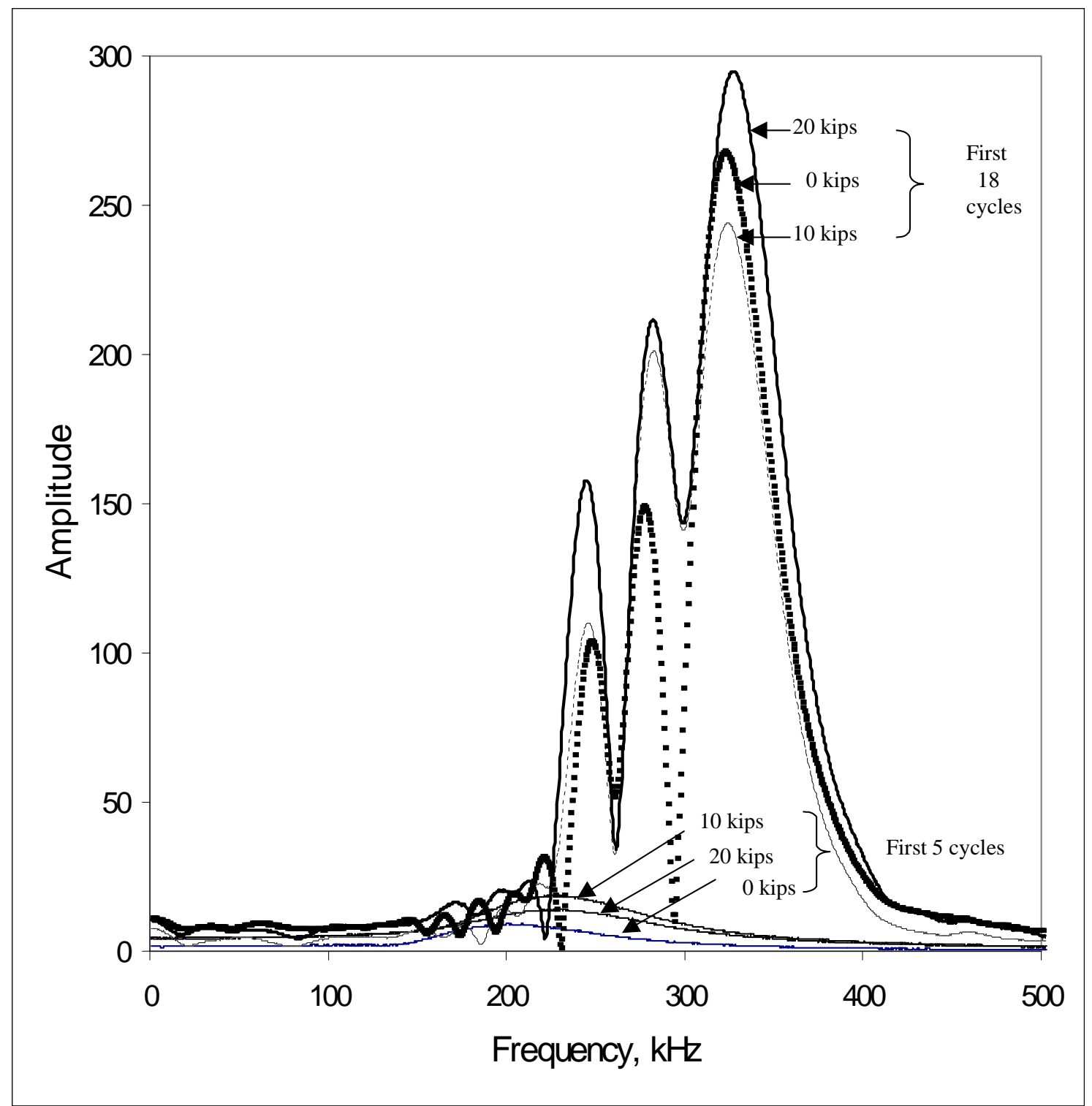

Figure 4.6 Frequency components of the first 5 and the first 18 cycles of the waveforms due to different tensile forces. 


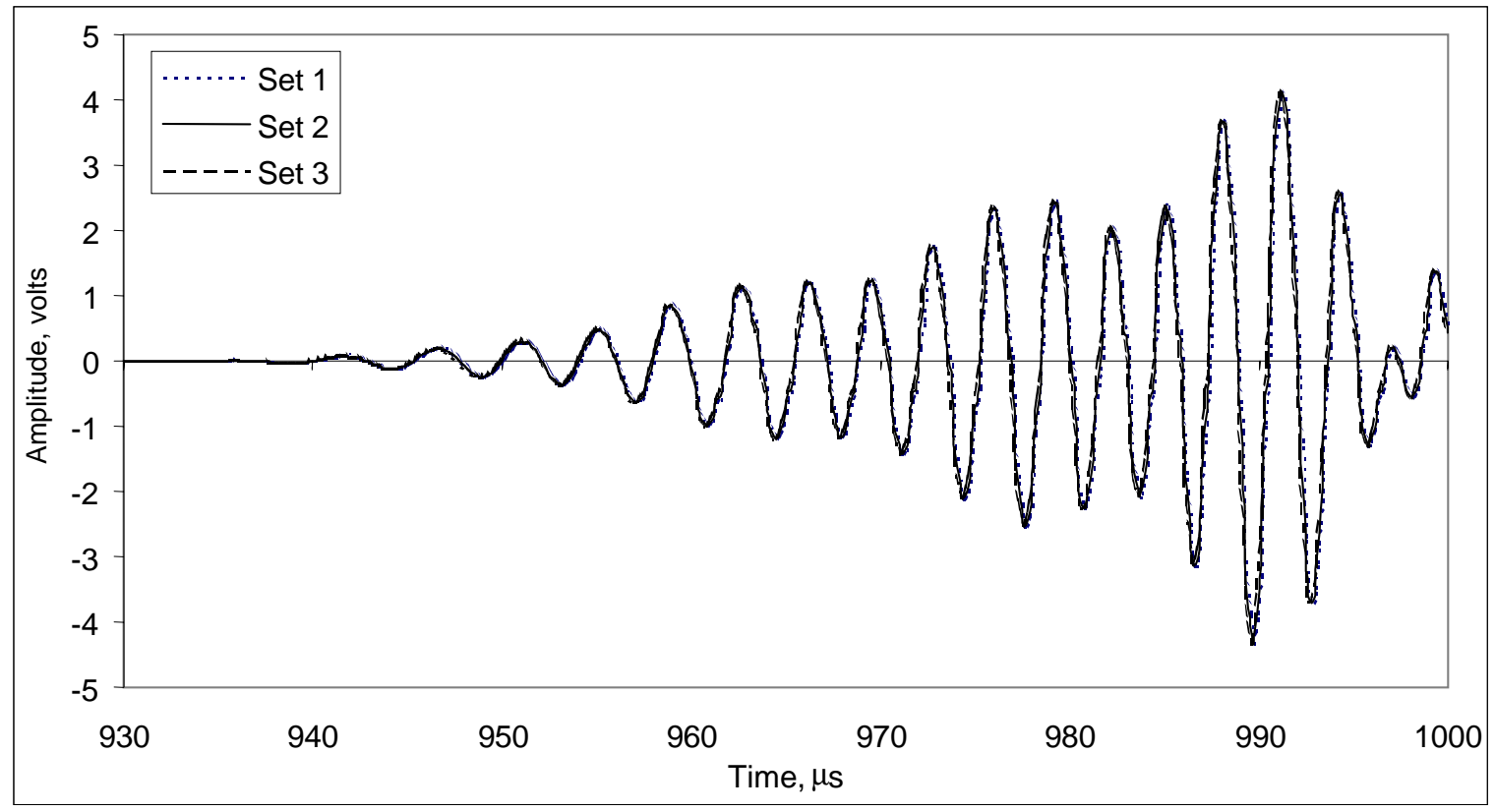

Figure 4.7 Arrival part of the waveform due to 0 kips tensile force. 


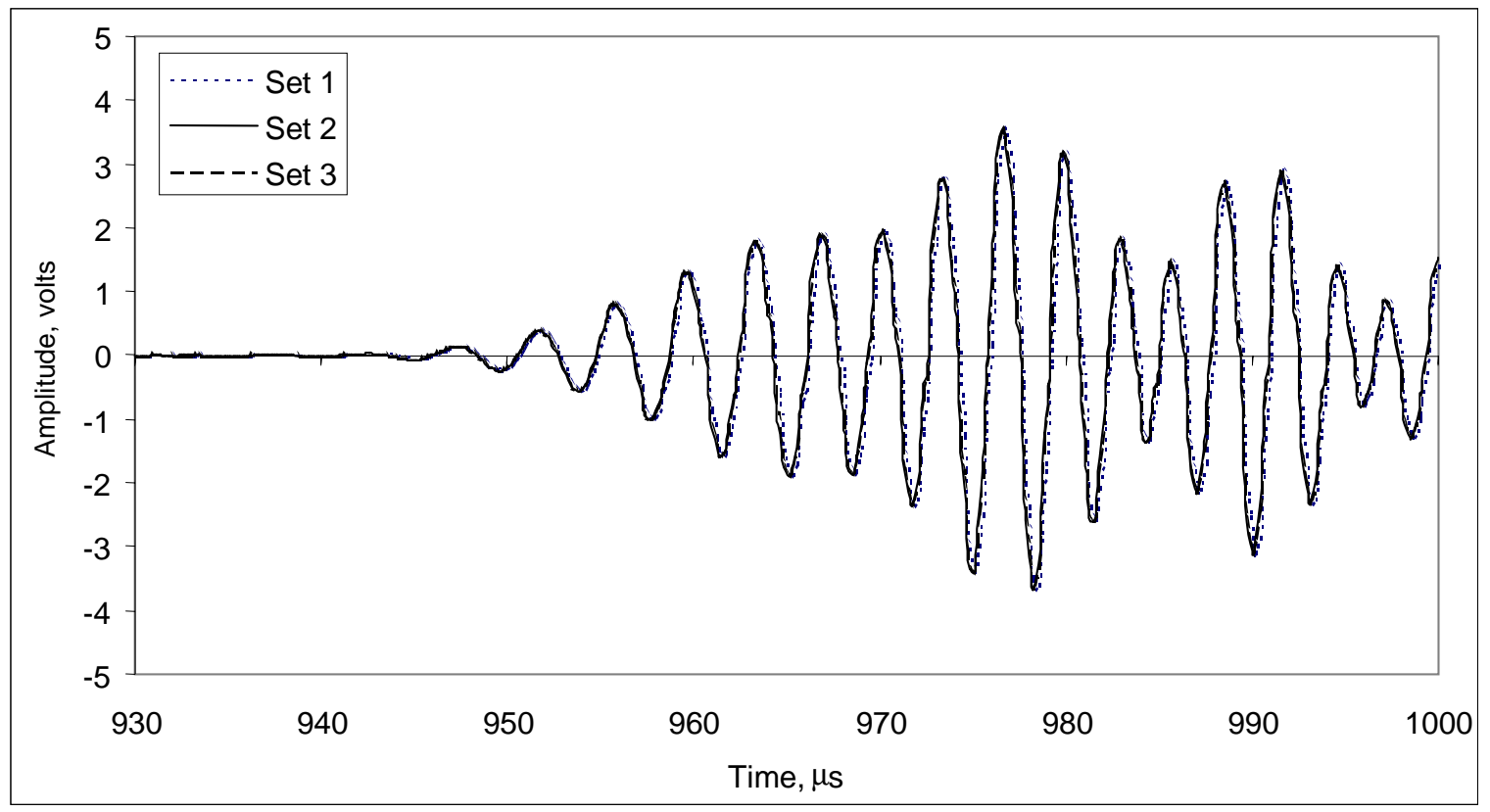

Figure 4.8 Arrival part of the waveform due to 10 kips tensile force. 


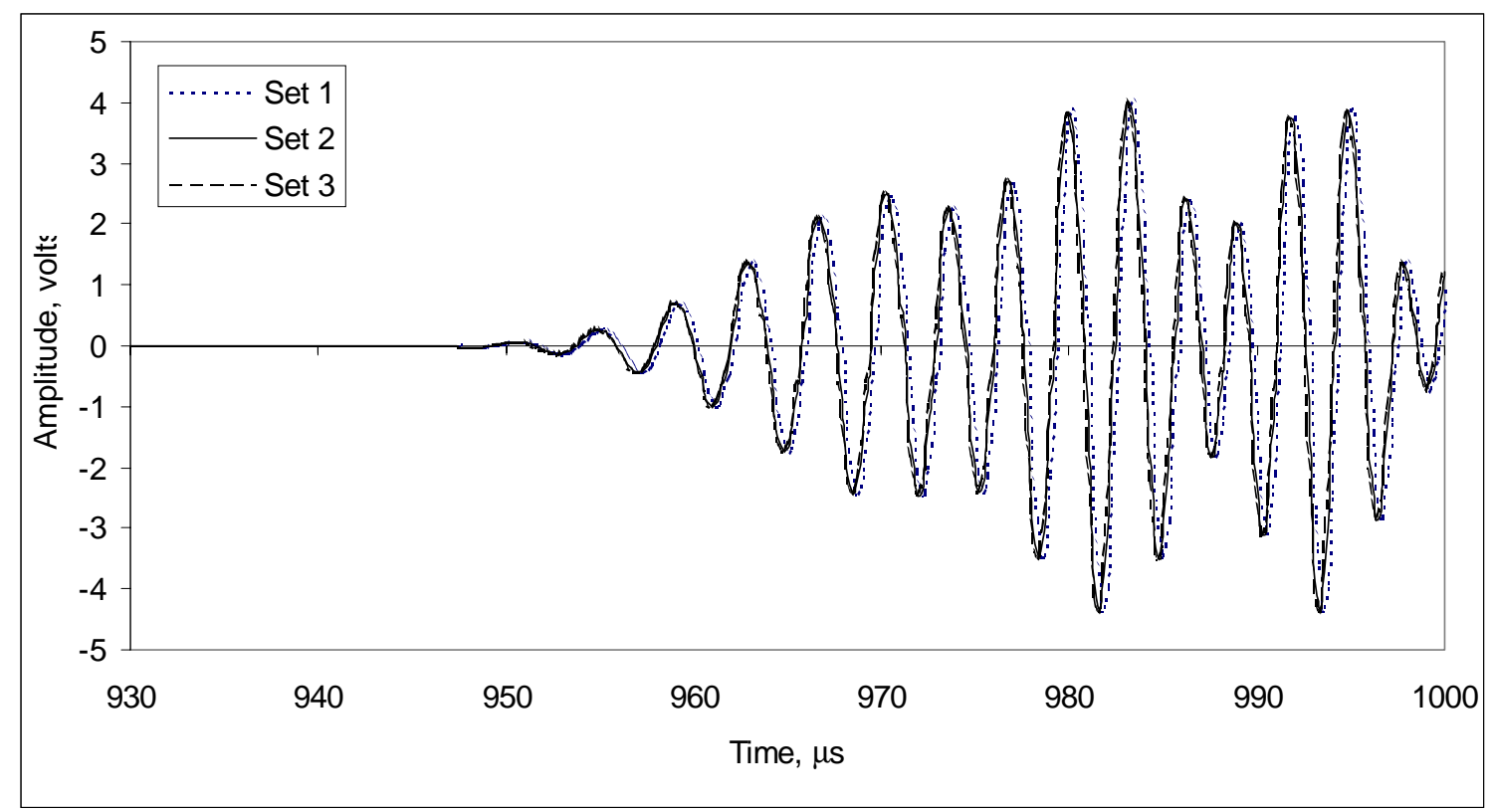

Figure 4.9 Arrival part of the waveform due to 20 kips tensile force. 


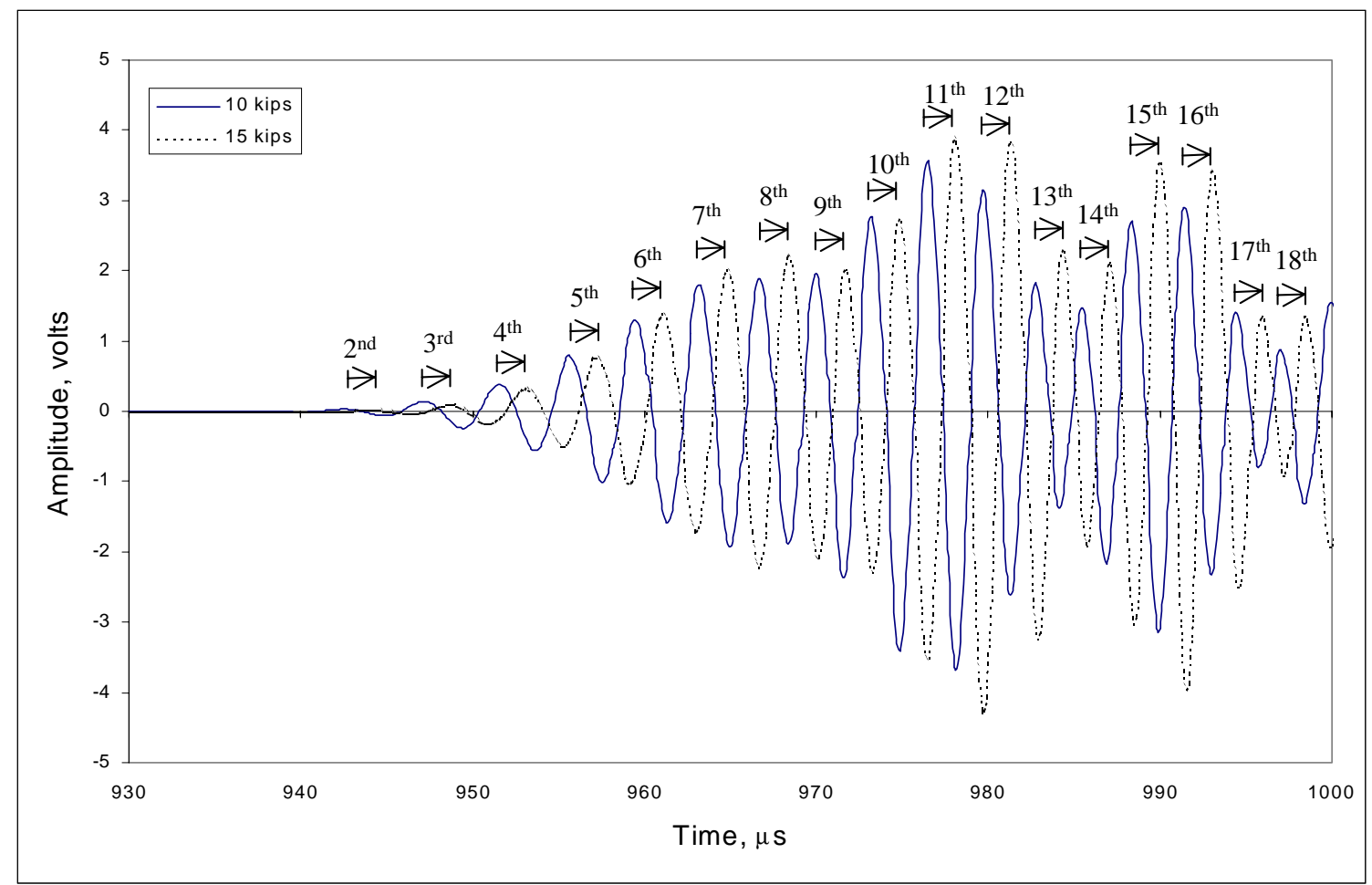

Figure 4.10 Time shift of waveforms due to an increase in tensile force from 10 kips to 15 kips. 


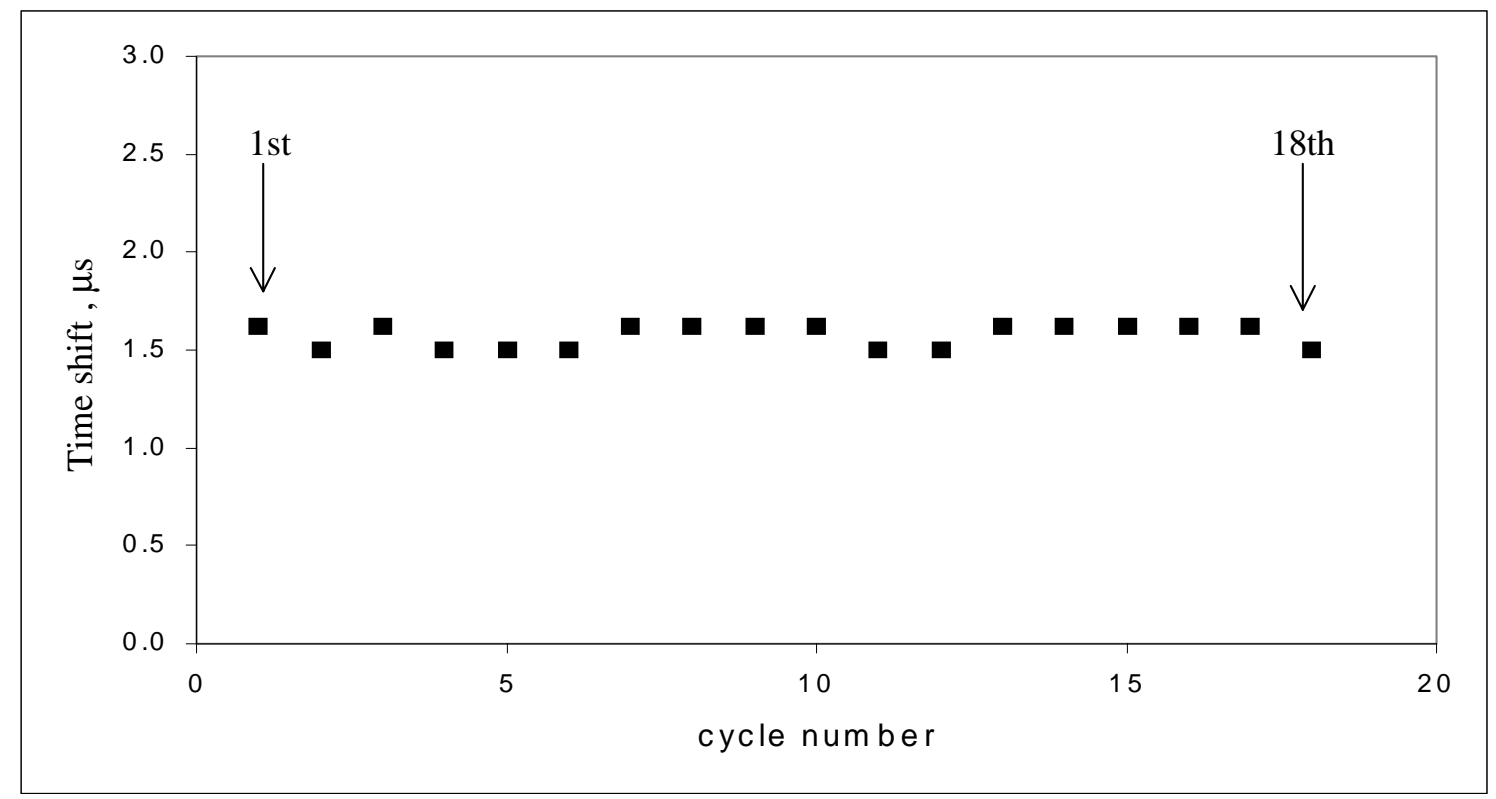

Figure 4.11 Time shift of the first 18 cycles of the waveform due to an increase in tensile force from 10 kips to 15 kips. 


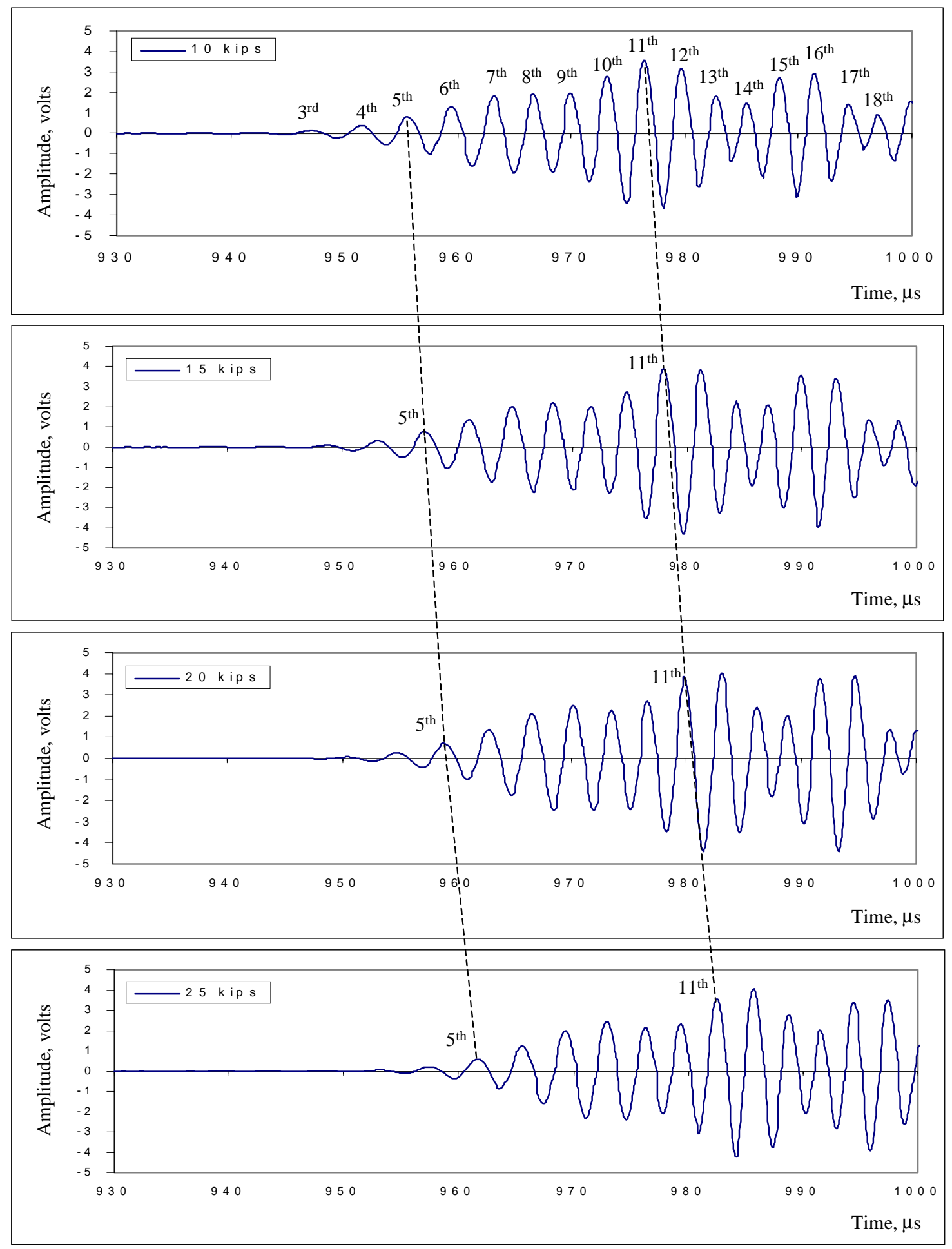

Figure 4.12 Time shift of the waveforms due to changes in tensile force. 


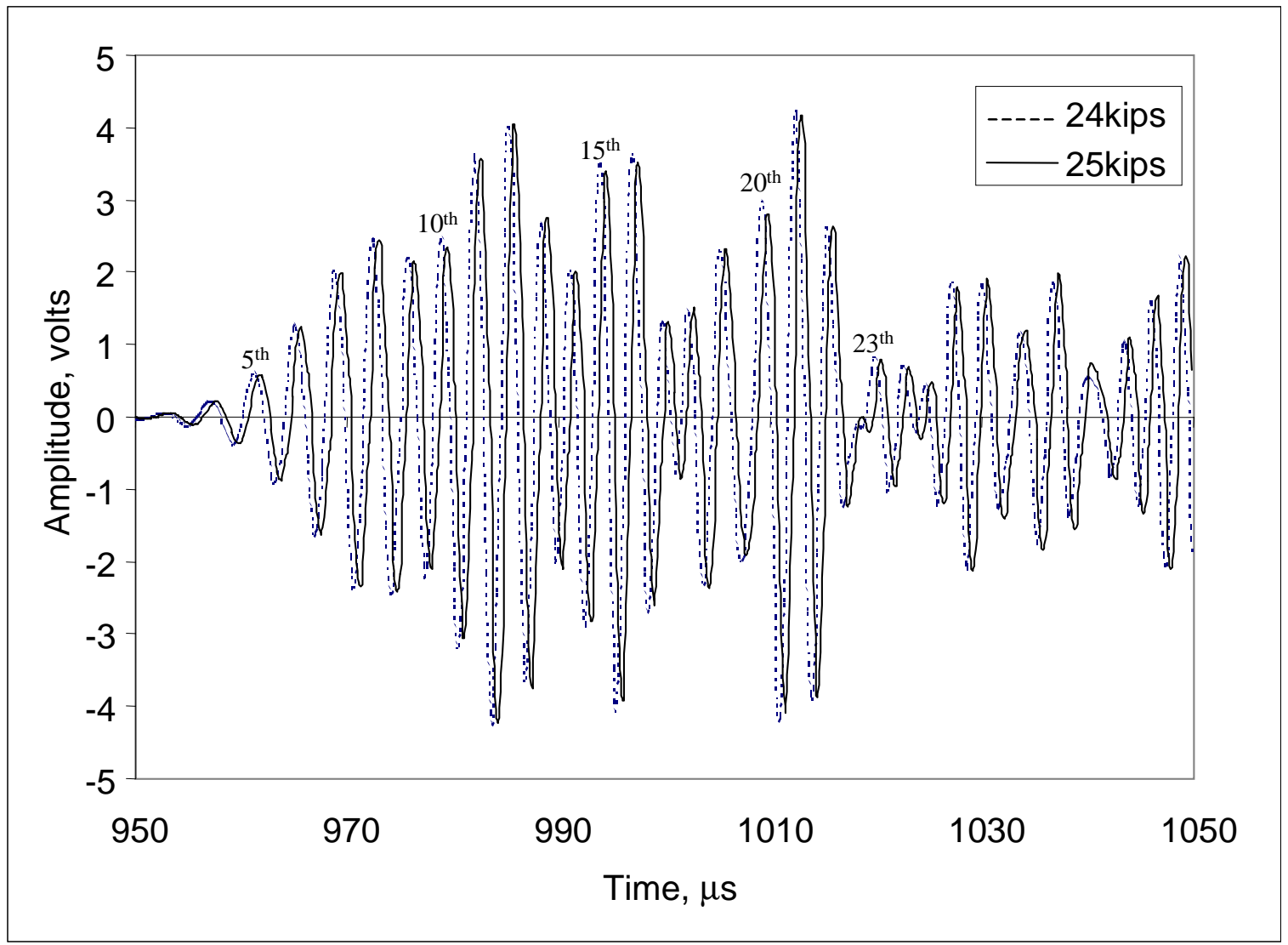

Figure 4.13 Comparison of shape and amplitude between waveforms recorded at 24 kips and 25 kips tensile forces. 


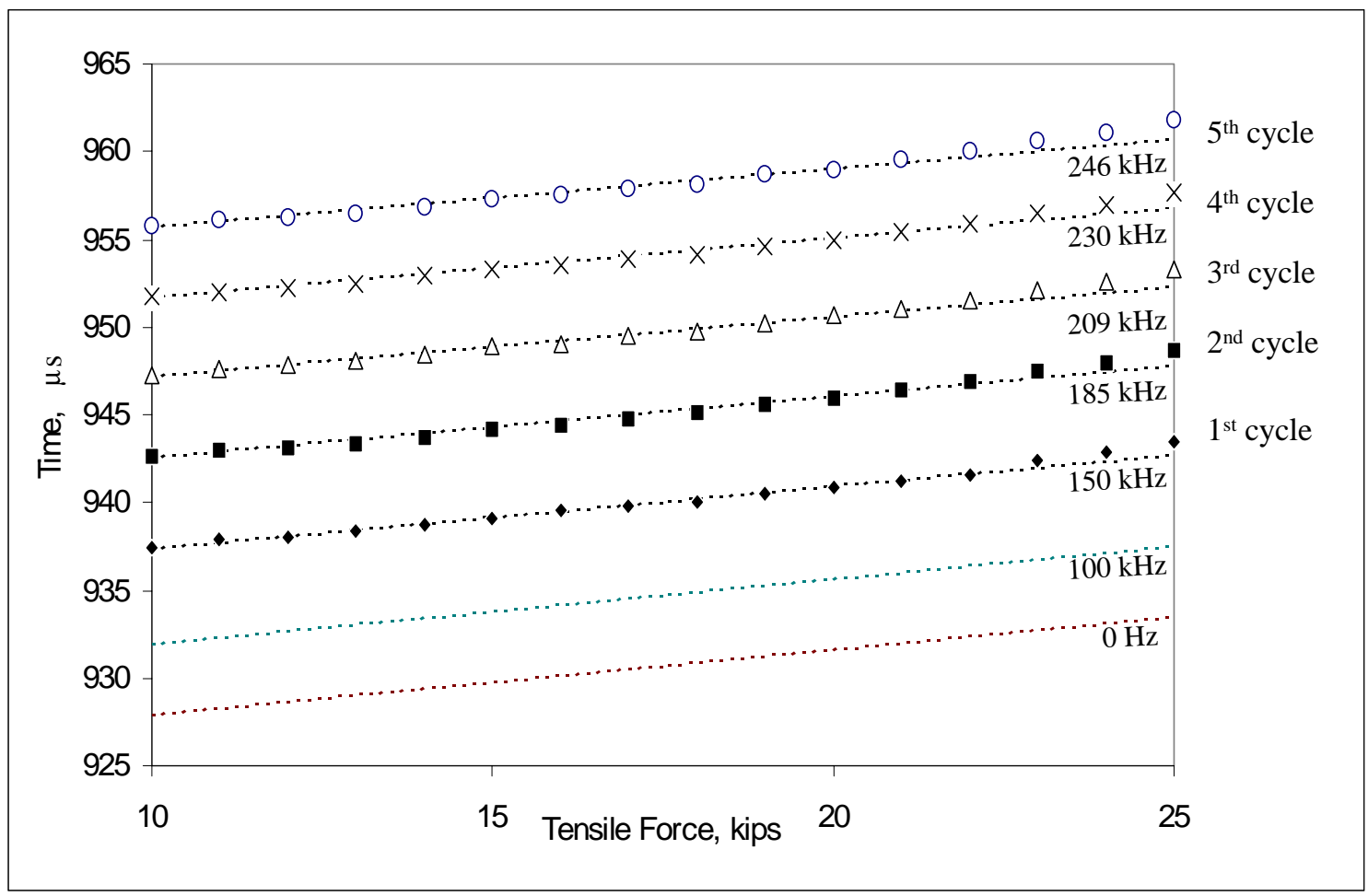

Figure 4.14 Peak arrival times of the first five cycles of the waveforms. Dotted lines represent the analytical approximations. Different types of markers are used to represent experimentally observed peak arrival times. 

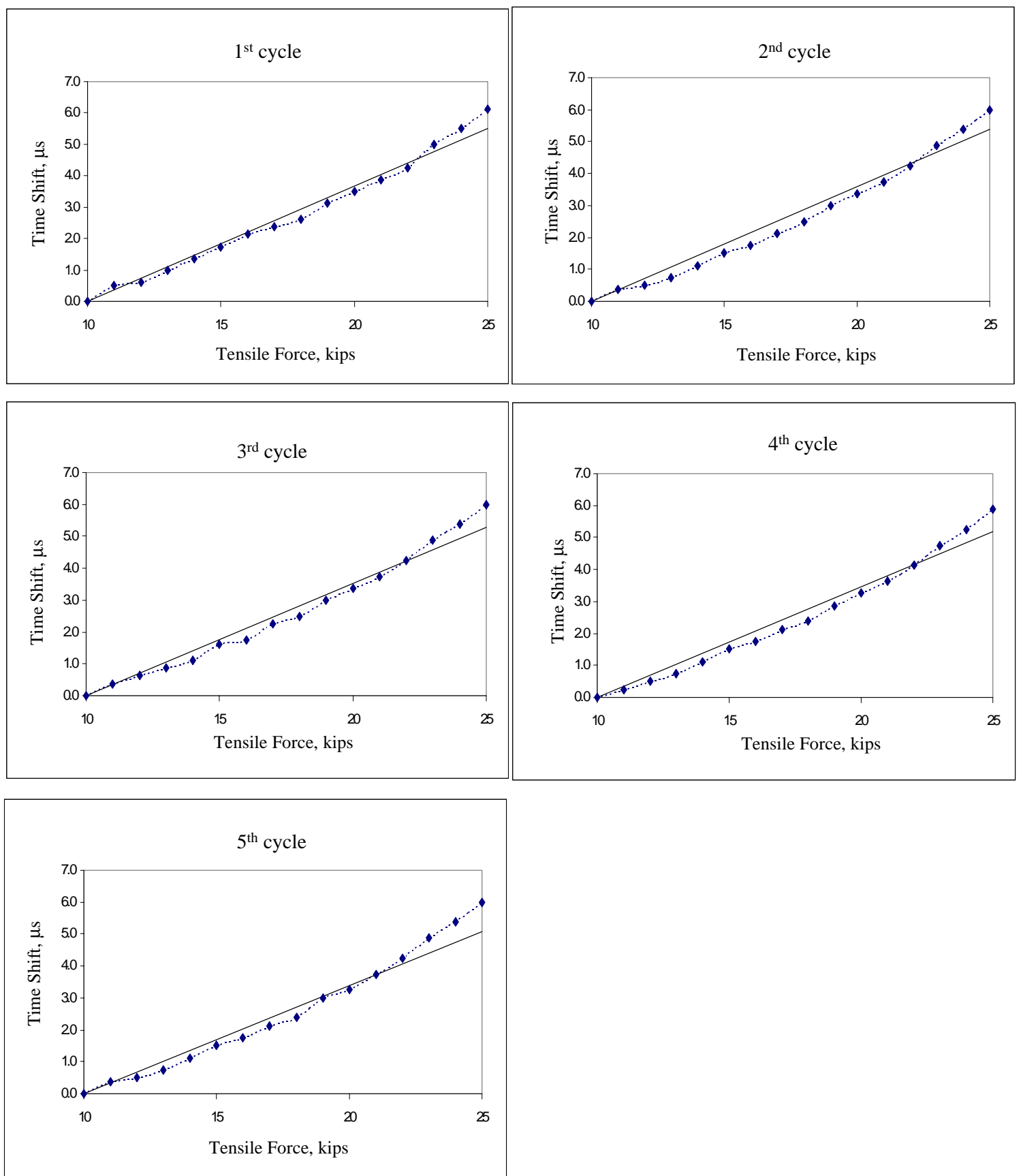

Figure 4.15 Time shift comparison between experimental (dotted lines) and analytical results (solid lines) for the first 5 cycles of the waveforms. 


\section{CHAPTER 5}

\section{APPLICATION OF THE WIGNER-VILLE TRANSFORM TO EVALUATE TENSILE FORCES IN SEVEN-WIRE PRESTRESSING STRANDS}

\subsection{OVERVIEW}

Theoretical and experimental studies in this chapter are focused on the improvement of the proposed stress wave technique by introducing a signal-processing tool, namely the Wigner-Ville Transform. As described in the previous chapter, the single frequency assumption was made in order to approximate the frequency component constituted in each peak of the recorded waveform. The relative change in tensile force in the strand was determined by measuring time shift of the waveforms. As seen in Figure 4.12, a forward time shift of each peak of the waveforms, due to increasing tensile forces, can be observed. However, identifying the same peaks among these waveforms can be difficult, sometimes, because of changes in the waveform's shape and amplitude. For instance, the peak amplitude is at the $11^{\text {th }}$ cycle for the tensile force of $10 \mathrm{kips}$, but it gradually shifts to the $12^{\text {th }}$ cycle when the tensile force is increased to 25 kips. Nevertheless, this method still requires one waveform to be recorded for each strand right after the initial jacking force was released so that it can be used as a referenced waveform when compared to be compared with waveforms recorded in future measurements. Also, the material properties of the strands 
need to be given. In addition, when the measurements are conducted at different times, matching the same cycle of different waveforms can be difficult due to changes of several factors such as conditions of the strands, interface between transducers and strands, and background noise level. The cycle matching will also be difficult in a longer strand because the same cycles among these waveforms will locate farther apart in time and will be difficult to match. To overcome these difficulties, a time-frequency representation technique is introduced in this chapter.

\subsection{WIGNER-VILLE TRANSFORM}

Time-frequency representations (TFRs) are useful tools for characterizing the behavior of signals whose spectral characteristics are time-varying. Because of their usefulness, TFR's have been applied to a wide variety of problems in signal processing. Most notably, they have been used for signal recovery at low signal-to-noise ratios. Accurate estimation of instantaneous frequency and group delay, signal detection in communications, radar, sonar application, and the design of time-varying signals and filters (Griffith et al., 1997). The Wigner-Ville Transform (WVT) is one of the most commonly-used TFR's. The WVT is a natural extension of Fourier analysis. It provides a more complete representation of signal behavior, particularly in the case of dispersive signals presented in this study.

By taking into account of the acoustoelastic effect and dispersive behavior of the stress waves, it is possible to estimate the tensile force in the prestressing strand by measuring the traveling time of one particular frequency component of the detected 
waveform. The WVT technique was employed as a mathematical tool for analyzing signals with time-varying spectra. The WVT of transient signals provides a method for mapping the frequency spectrum as a function of time. The WVT can be regarded as the Fourier transform of the signal's autocorrelation function with respect to the delay variable $(\tau)$. The WVT of a signal $x(t)$ is given by

$$
W_{x}(t, f)=\int_{\tau} x\left(t+\frac{\tau}{2}\right) x^{*}\left(t-\frac{\tau}{2}\right) e^{-i 2 \pi f \tau} d \tau
$$

where $x^{*}()$ is the complex conjugate of $x(t)$. The term $x() x^{*}()$ is the time-dependent autocorrelation function taken at time $t$. The variable $\tau$ denotes the time interval separating the sampling times, which are located symmetrically about the absolute time, $t$.

One of the desirable mathematical properties of the WVT is its marginal property that can be expressed as

$$
\int_{-\infty}^{+\infty} W_{x}(t, f) d f=|x(t)|^{2} \quad \text { and } \quad \int_{-\infty}^{+\infty} W_{x}(t, f) d t=|X(f)|^{2} .
$$

From equation (5.2), it is shown that the WVT for any time $t$ can be regarded as a decomposition in the frequency $f$ of the instantaneous power $|x(t)|^{2}$. Similarly, for any value of $f$, the WVT can also be regarded as a decomposition in time $t$ of the energy spectrum $|X(f)|^{2}$.

The WVT is a particularly useful presentation of the behavior of the stress waves because its outputs are in real numbers which also act as a density function describing the 
concentration of the energy of the signal over the time-frequency plane. The group velocity is also defined as energy propagation velocity of the wave motion at a particular frequency. Hence, using the WVT to measure the arrival time of each frequency component of the recorded waveforms was applied in this study. A WVT software developed by Auger et. al. (1995) was used to analyze the recorded waveforms. The WVT results are described in the following section.

\subsection{LABORATORY EXPERIMENTS}

A schematic drawing of the experimental setup is shown in Figure 5.1. A total of three $1 / 2$ inch diameter prestressing strands (one of Grade 250 and two of Grade 270) were tested. There was only one strand tested at a time. For each test, the strand was placed in a hollow core inside a loading frame made of three reinforced concrete beams. The strand was not bonded with concrete. The instrumentation of this experiment is the same as the one described in the previous chapter where tensile force was gradually applied to the strand by a hydraulic jack. The maximum applied tensile force was 28 kips. A load cell was used as load indicator. Two steel chucks were used to grip the strand at both ends. The total length of each strand is $12.35 \mathrm{~m}(40.52 \mathrm{ft})$ and the actual length under tension is $11.69 \mathrm{~m}(38.35 \mathrm{ft})$.

The sensing equipment and experimental procedures in this experiment are the same as those described in the previous chapter. The pulse generator was used to generate the wave motions through the input transducer. Wave motions in the longitudinal direction were detected by the output transducer. Ultragel II was used as a coupling agent. The 
MISTRAS system was used to record the incoming waveforms. Multiple sets of data at each tension level were recorded using $8 \mathrm{MHz}$ sampling rate.

\subsection{EXPERIMENTAL RESULTS AND DISCUSSIONS}

\section{Grade 250 strand}

Figure 5.2 shows arriving waveforms due to 15 kips and 20 kips tensile forces. It is observed that, when the strand is subjected to 15 kips tensile force, the waveform in this figure has its peak amplitude at about $2500 \mu \mathrm{s}$. It is also observed in Figure 5.2 that, when the tensile force is increased from 15 kips to 20 kips, the average forward time shift of the same peak amplitude of the waveforms is about $0.43 \mu \mathrm{s} / \mathrm{kip}$. By applying the Fourier transform technique, frequency components constituted in this waveform due to 15 kips tensile force are shown in Figure 5.3. Results show that the frequency components of the waveform constitute mainly from $270 \mathrm{kHz}$ to $330 \mathrm{kHz}$ with the peak frequency of about 300 $\mathrm{kHz}$. It is also found that the frequency components are similar at all tension levels.

Consistent WVT results were obtained for each and every tension level. As shown in Figure 5.4 - Figure 5.6, three waveforms subjected to different tensile forces of 15 kips, 20 kips, and 25 kips were analyzed. Similar to the results obtained from the previous chapter, WVT results clearly illustrate the dispersive behavior of the waves such that, for the first longitudinal mode appeared in this frequency range (Figure 5.3), the lower frequency components would arrive earlier than the higher frequency components. Also shown in these figures, amplitudes of the frequency components are represented by distinct closed island shape contours. For example, the WVT result plotted in Figure 5.4 has its peak 
frequency of $300 \mathrm{kHz}$ at about $2500 \mu \mathrm{s}$. It, therefore, corresponds well with the previous results in time domain (Figure 5.2) and in frequency domain (Figure 5.3). Thus, the WVT technique shows its ability of providing the information of the recorded waveform simultaneously in time and frequency domains.

According to the previously given assumption, the waveform would constitute only one frequency component at each particular point in time. However, the WVT results show a narrow band of frequencies within the monitoring time period. As shown in Figure 5.4Figure 5.6, the arrival times of different frequency components of the waveforms can be approximated by a ridge line, which passes through the center of each WVT contour.

The value of $C_{g}$ is dependent on propagating frequency and material constants of the strand $(\rho, \lambda, \mu, l, m$, and $n)$. The second-order constants can be determined by the customary longitudinal and transverse wave-speed measurements. The third-order constants can be determined by measuring relative changes in wave speeds in three directions (Egle and Bray, 1976). Due to limitation of our laboratory facility, the above methods cannot be followed. Therefore, an indirect method of determining the material constants was developed in this study where the WVT results due to different tensile forces were used to determine the values of the material properties. By using the trial-and-error method, all of these values were initially assumed and were then used to calculate the group velocity and arrival time of each frequency component. These material constants (Table 5.1) were numerically adjusted in order to provide the best fit to the ridge lines at each and every tension level. The material constants obtained using the indirect method are only the approximation of the actual material properties of the prestressing strand. These material constants are estimated 
using the single frequency approximation and assuming traction free boundary conditions of the center wire in the analysis.

Numerical study of the variation of the material properties was conducted and the results are shown in Table 5.2. Table 5.2 shows the variation of the traveling time of the 300 $\mathrm{kHz}$ frequency component due to $\pm 1 \%$ change in each of the material constants while other material properties remain constant. In this analysis, the strand is subjected to 28 kips tensile force. It is shown that these material constants unequally affect the group velocity $\left(C_{g}\right)$ of the propagating waves. In other words, they unequally affect the estimation of the tensile force. Among these material constants, the mass density $(\rho)$ and the shear modulus ( $\mu$ ) have the most influence on $C_{g}$. As described by equation (4.3) and equation (4.4), the change of $\rho$ would directly affect the wave velocities $\left(C_{L}\right.$ and $\left.C_{T}\right)$, and would consequently affect the value of $C_{g}$. By examining the frequency equation, changes of $\mu$ value will have the strongest effect on $C_{T}$, especially in the $(y-2 x)^{2}$ term of equation (4.10) where its fourth power is presented $\left(y=\omega^{2} a^{2} / C_{T}^{2}\right) . \quad \lambda$ value also has an effect on $C_{L}$, but only the first power of $C_{L}$ is presented in the $\sqrt{\left(y / r^{2}\right)-x}$ term of this equation $\left(y / r^{2}=\omega^{2} a^{2} / C_{L}^{2}\right)$. Numerical results shown in Table 5.2 confirm that $\lambda$ has less effect on $C_{g}$ than $\mu$. As described by equation (4.6) and equation (4.7), although there are infinite terms containing higher powers of $C_{L}$ and $C_{T}$ presented in the Bessel functions, each term becomes much smaller as its power increases. Among the third-order elastic constants, $m$ has the most effect on $C_{L}, C_{T}$, and $C_{g}$ followed by $n$ and $l$, in descending order. In determining material constants using the trialand-error method, each individual constant was adjusted accordingly. In practical cases, these material constants may be varied from one manufacturer to another due to different 
chemical composition, manufacturing process, and quality control. Investigation of these factors should be conducted in the future.

Numerical analysis based on the frequency equation was conducted to provide dispersion curves of a stress wave propagating in the center wire. Figure 5.7 shows dispersion curves of the first two longitudinal modes of a stress wave propagating in the strand. Results confirm that the frequency components of the recorded waveforms belong to the first longitudinal mode. Figure 5.8 shows the calculated and the measured arrival times of each frequency component of the waveforms due to three different tension levels. The solid lines represent the calculated arrival times of the frequency components of the first longitudinal mode based on the strand's material constants provided in Table 5.1. The dotted lines represent the experimental results (ridge lines). Figure 5.8 also shows the forward time shifts of each frequency component due to increasing tensile forces from 15 kips to 25 kips. There is only a slight variation of the slopes among the ridge lines of different tensile forces. This is because these parts of the waveforms contain frequency components within a narrow range. The changes of group velocities among these frequency components are still very small. Hence, the average forward time shift is about constant, which is approximately 0.43 $\mu$ s per 1 kip increment of the tensile force. The average time shift is about the same as the one previously shown in Figure 5.2 where the time shift of the same cycle of the waveforms was measured.

The sampling rate of $8 \mathrm{MHz}$, providing a time measurement resolution of $0.125 \mu \mathrm{s}$, is sufficient for recording the arrival parts of the waveforms, which have major frequency components of about $300 \mathrm{kHz}$. Since the average forward time shift is about $0.43 \mu$ s per 1 kip increment of the tensile force, the resolution of one data point per 0.29 kip was received. 
In comparison to the experimental results presented in Figure 5.8, it was found that the predicted arrival time error of each frequency is less than $0.2 \mu \mathrm{s}$. Thus, the corresponding error in tensile force prediction is less than 0.47 kip or $3.26 \mathrm{ksi}$, which is approximately 1.3 percent of the maximum strength of the strand $(250 \mathrm{ksi})$. Therefore, the material constants obtained by the trial-and-error method can effectively be used to calculate the arrival time of each frequency component.

\section{Grade 270 strands}

The same experimental setup was used to test two $1 / 2$ " diameter prestressing strands of Grade 270. These strands were cut from the same reel in order to investigate the consistency of the results. Test results from the first strand are shown in Figure 5.9 - Figure 5.11. In these figures, three waveforms subjected to different tensile forces of 12 kips, 16 kips, and 20 kips were analyzed. WVT results show the dispersive behavior of the waves within $260 \mathrm{kHz}$ to $310 \mathrm{kHz}$ frequency range of the first longitudinal mode, which is similar to the one obtained from the previous test (Grade 250 strand). A ridge line was drawn in each WVT plot to locate the arrival time of each frequency component of the waveform. The trial-anderror method of determining the material constants was conducted and the material constants of the strand were calculated (Table 5.3). Numerical analysis based on the frequency equation was conducted and the dispersion curves of the first two longitudinal modes are presented in Figure 5.12.

Figure 5.13 shows the calculated and the measured arrival times of each frequency component of the waveforms due to 12 kips, 16 kips, and 20 kips tensile forces. The solid lines represent the calculated arrival times of the frequency components of the first 
longitudinal mode based on the strand's material constants shown in Table 5.3. The dotted lines represent the experimental results. Consistent WVT results were obtained for each and every tension level. The average forward time shift is $0.45 \mu$ s per 1 kip increment of the tensile force.

The result from the second strand (Grade 270) is shown in Figure 5.14. This figure shows the WVT contour and its corresponding ridge line (solid line) due to 28 kips tensile force. The calculated time-frequency relationship of the first strand (dotted line) is also shown in this figure. The result comparison shows some that there are differences in time and frequency relationship between the two ridge lines. For instance, it was found that the difference in arrival time error of $300 \mathrm{kHz}$ component is approximately $3.8 \mu \mathrm{s}$. Thus, the corresponding error in tensile force prediction is $8.4 \mathrm{kips}$, or about $54.9 \mathrm{ksi}$, which is about 20 percent of the maximum strength of the strand $(270 \mathrm{ksi})$. The error among these results may be due to the variation of the strand's properties even though these strands were taken from the same reel. A greater number of strands should be tested to investigate the variation of the strand's material properties. Another cause of such error may possible due to an inaccurate measurement of the strand length. For example, the calculated group velocity of the $300 \mathrm{kHz}$ component due to $28 \mathrm{kips}$ tensile force is $4925 \mathrm{~m} / \mathrm{s}$. An error of \pm 0.5 inch ( $0.1 \%$ of the total length) in the strand length measurement would produce $\pm 2.6 \mu \mathrm{s}$, or \pm 5.8 kips error in tensile force measurement. From the WVT result comparison, the first strand may be slightly shorter than the second strand. Corresponding results due to 20 kips tensile force (Figure 5.14a) show that the first cycle of the waveforms was detected earlier at the receiving end of the first strand. Thus, the difference of WVT results between these two strands may likely be due to the error in the strand length measurement. In order to avoid 
this problem, it is recommended that time shift of the waveforms for each individual strand should be measured instead. As shown in Figure 5.14b, time shift of the waveforms recorded at three different tension levels in each strand was measured. For the first strand, the average time shift of the waveforms due to a tensile force increment from 12 kips to 20 kips is about $0.31 \mu \mathrm{s} / \mathrm{kip}$. For the second strand, the average time shift of the waveforms due to the tensile force increment of the same range is about $0.28 \mu \mathrm{s} / \mathrm{kip}$. Therefore, the time shift measurement of these waveforms provides more consistent results for both strands. By using this technique, the measurement should be first conducted immediately after the initial jacking force was released so that waveforms due to a known tension level are recorded, as reference waveforms. Then, waveforms recorded in the future can be compared with the reference waveforms so that time shift of any particular cycle can be measured. Thus, changes in tension level of the strand can be calculated.

\subsection{SUMMARY}

In this chapter, both experimental measurements and theoretical analysis were conducted. Three 1/2-inch diameter seven-wire prestressing strands of Grade 250 and Grade 270 were tested. A numerical calculation considering the elastic waveguide theory and the acoustoelastic effect was conducted. The waveform analysis using the Wigner-Ville Transform provides a successful description of the behavior of a longitudinal transient wave traveling in the center wire. Material properties of the strands were indirectly determined from the measured stress waveforms using the single frequency approximation. The numerical results correlate well with the experimental observation and high measurement accuracy of the tensile forces in the strands was observed. 


\begin{tabular}{|c|cc|}
\hline & GRADE 250 \\
\hline$\rho$ & $=7.300 \times 10^{-4} \quad\left(\mathrm{lb}-\mathrm{s}^{2} / \mathrm{in}^{4}\right)$ \\
\hline$\lambda$ & $=13.358 \times 10^{6} \quad(\mathrm{psi})$ \\
\hline$\mu$ & $=11.8545 \times 10^{6} \quad(\mathrm{psi})$ \\
\hline 1 & $=-35.960 \times 10^{6} \quad(\mathrm{psi})$ \\
\hline $\mathrm{m}$ & $=-62.425 \times 10^{6} \quad(\mathrm{psi})$ \\
\hline $\mathrm{n}$ & $=-69.837 \times 10^{6} \quad(\mathrm{psi})$ \\
\hline
\end{tabular}

Where $\rho$ is the mass density; $\lambda$ and $\mu$ are Lame's elastic constants. $1, \mathrm{~m}$, and $\mathrm{n}$ are Murnaghan's elastic constants.

The nominal cross-sectional area of the strand is $0.144 \mathrm{in}^{2}$.

Table 5.1 Calculated material constants of the seven-wire prestressing strand (Grade 250). 


\begin{tabular}{|c|c|c|}
\hline $\begin{array}{c}\text { Material } \\
\text { Constant }\end{array}$ & $\begin{array}{c}\text { Group Velocity Variation } \\
(\mathrm{m} / \mathrm{s})\end{array}$ & $\begin{array}{c}\text { Tensile Force Variation } \\
\text { (kips) }\end{array}$ \\
\hline$\rho( \pm 1 \%)$ & $\mp 26.89$ & \pm 31.81 \\
$\lambda( \pm 1 \%)$ & \pm 1.26 & $\mp 1.511$ \\
$\mu( \pm 1 \%)$ & \pm 26.33 & $\mp 31.12$ \\
$1( \pm 1 \%)$ & $\mp 0.01$ & \pm 0.023 \\
$\mathrm{~m}( \pm 1 \%)$ & $\mp 0.53$ & \pm 0.628 \\
$\mathrm{n}( \pm 1 \%)$ & $\mp 0.12$ & \pm 0.163 \\
\hline
\end{tabular}

Table 5.2 Variation of group velocity and tensile force due to $\pm 1 \%$ change of the strand's material constants. (evaluated at propagating frequency $=300 \mathrm{kHz}$, tensile force $=28 \mathrm{kips}, \mathrm{C}_{\mathrm{g}}=4957 \mathrm{~m} / \mathrm{s}$ ). The $1 / 2$-inch seven-wire strand Grade 250 is used. 


\begin{tabular}{|c|cc|}
\hline & GRADE 270 \\
\hline$\rho$ & $=7.300 \times 10^{-4} \quad\left(\mathrm{lb}-\mathrm{-}^{2} / \mathrm{in}^{4}\right)$ \\
\hline$\lambda$ & $=12.05 \times 10^{6} \quad(\mathrm{psi})$ \\
\hline$\mu$ & $=11.871 \times 10^{6} \quad(\mathrm{psi})$ \\
\hline 1 & $=-35.960 \times 10^{6}$ & $(\mathrm{psi})$ \\
\hline $\mathrm{m}$ & $=-56.589 \times 10^{6} \quad(\mathrm{psi})$ \\
\hline $\mathrm{n}$ & $=-103.50 \times 10^{6} \quad(\mathrm{psi})$ \\
\hline
\end{tabular}

Where $\rho$ is the mass density; $\lambda$ and $\mu$ are Lame's elastic constants. $1, \mathrm{~m}$, and $\mathrm{n}$ are Murnaghan's elastic constants.

The nominal cross-sectional area of the strand is $0.153 \mathrm{in}^{2}$.

Table 5.3 Calculated material constants of the seven-wire prestressing strand (Grade 270). 


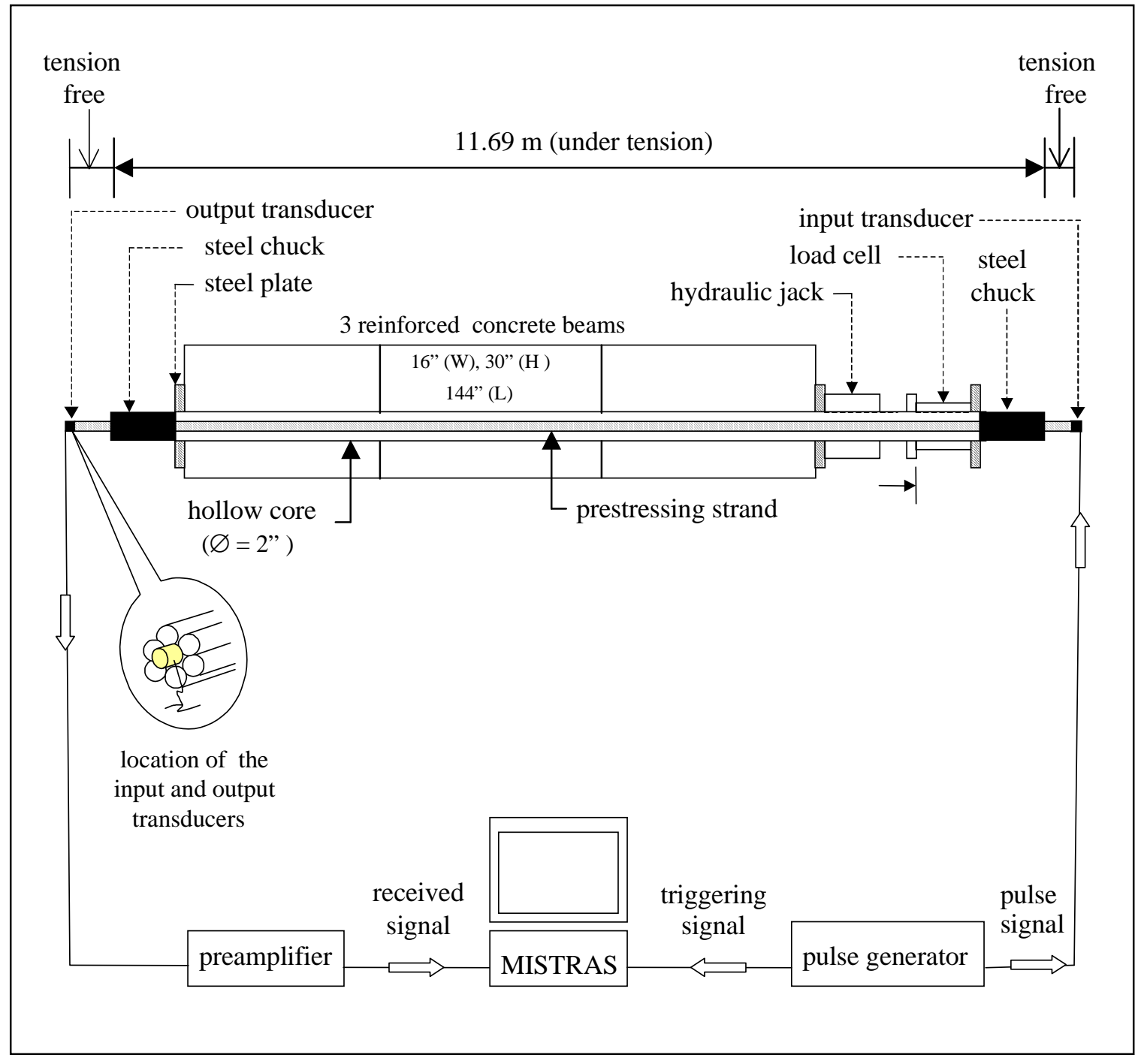

Figure 5.1 Experimental setup. 


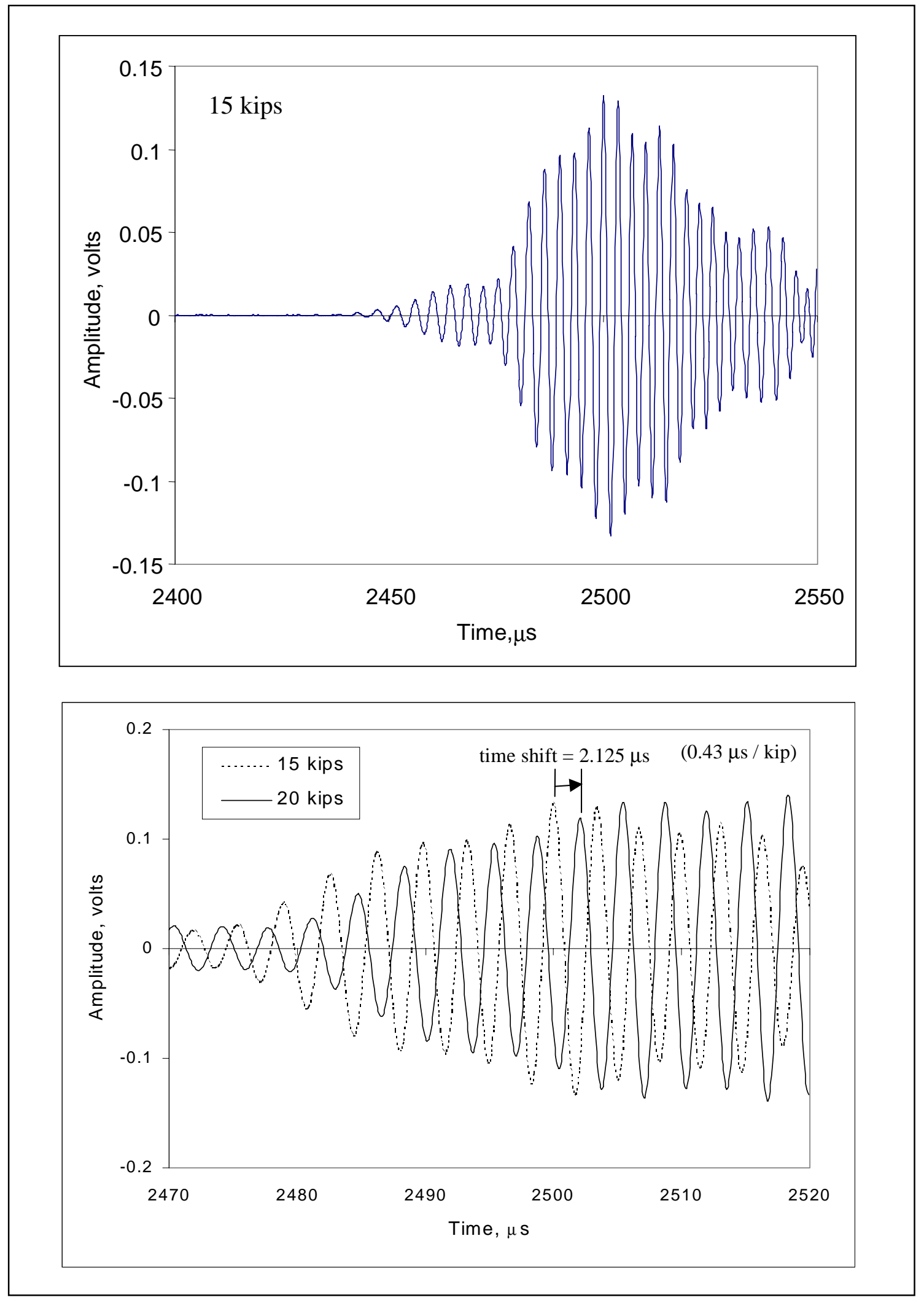

Figure 5.2 Recorded waveforms due to 15 kips and 20 kips tensile forces. The $1 / 2$-inch seven-wire strand Grade 250 is used. 


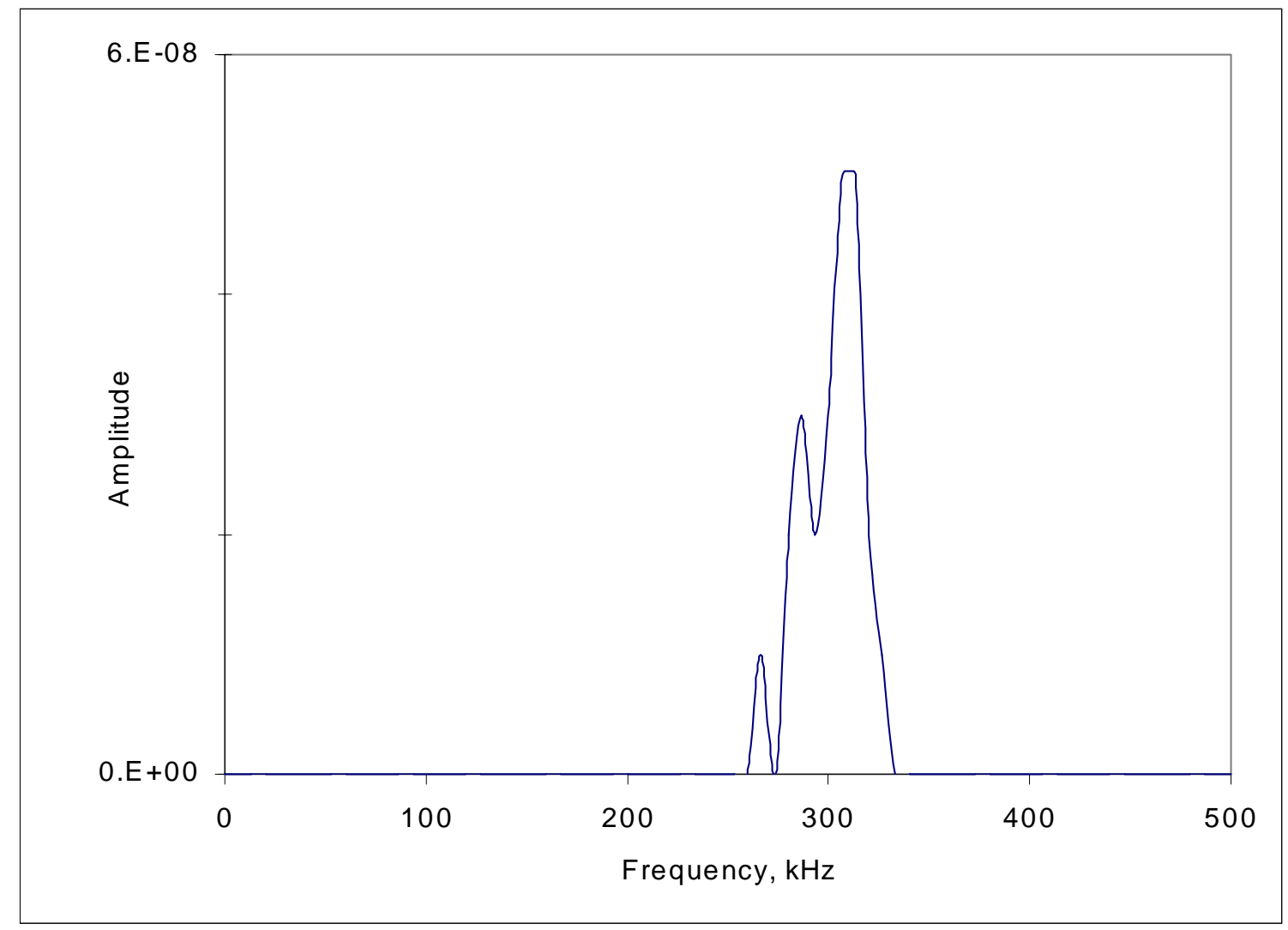

Figure 5.3 Frequency components of the recorded waveform due to 15 kips tensile force. The $1 / 2$-inch seven-wire strand Grade 250 is used. 


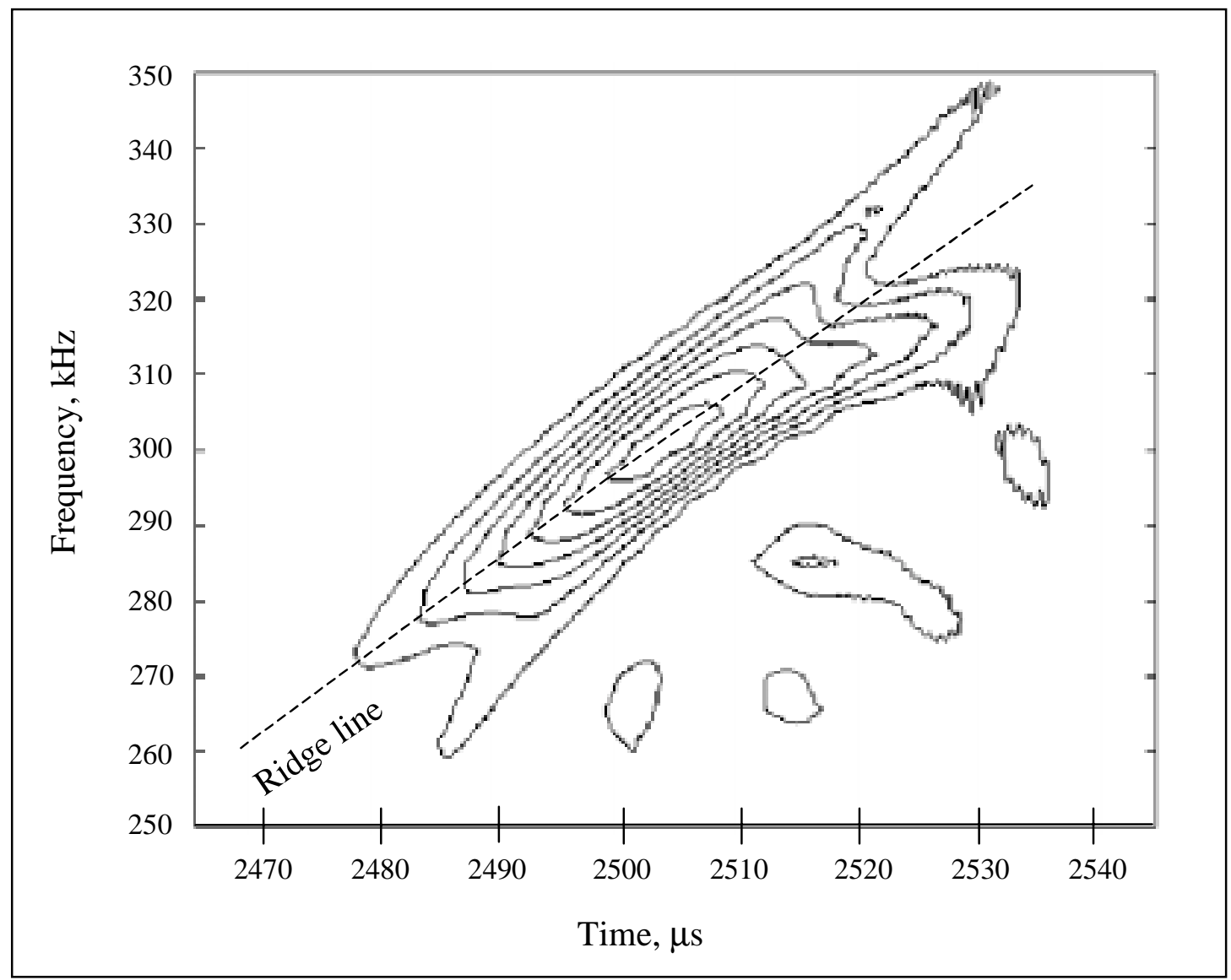

Figure 5.4 Time-frequency relationship of the recorded waveform due to $15 \mathrm{kips}$ tensile force. The $1 / 2$-inch seven-wire strand Grade 250 is used. 


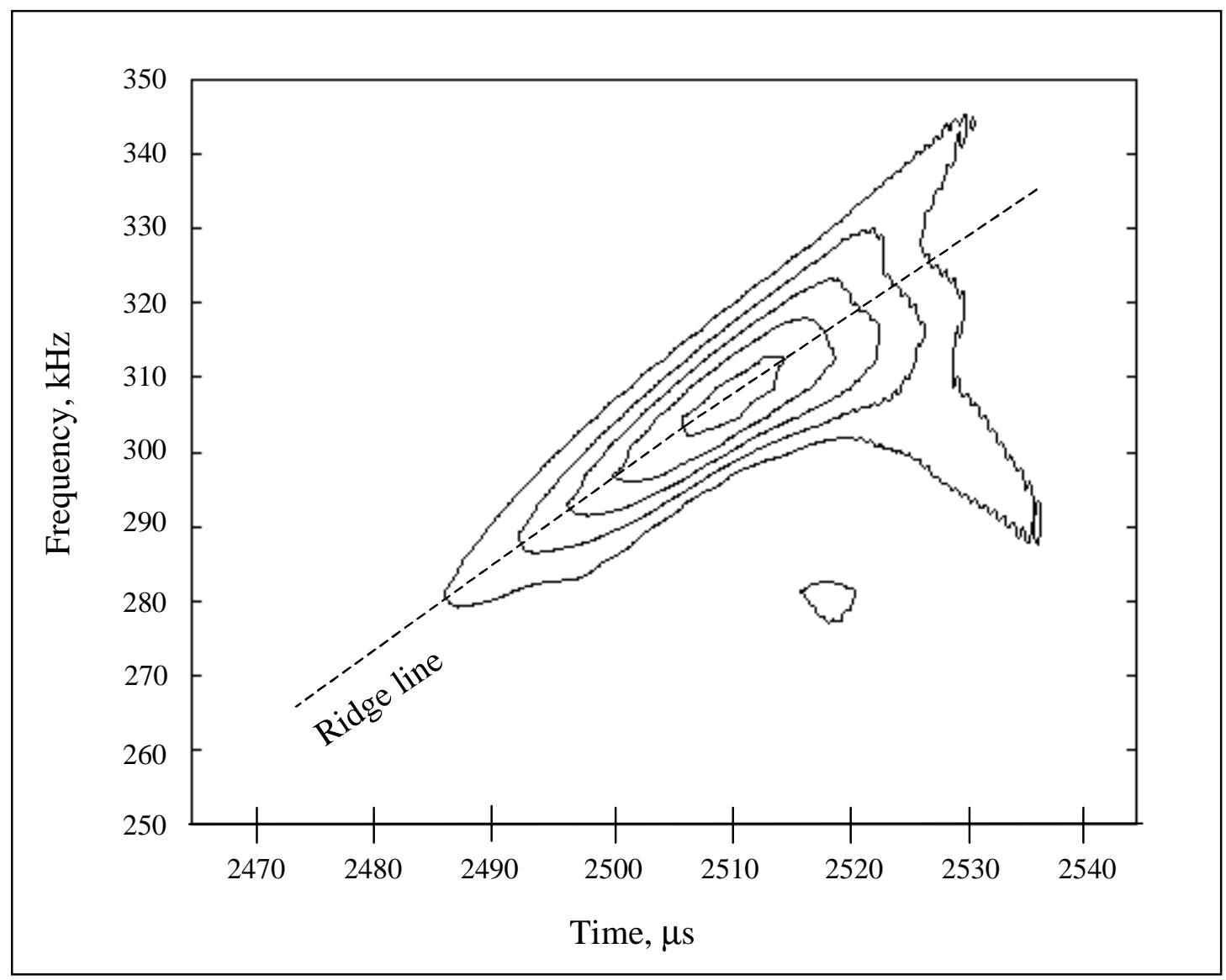

Figure 5.5 Time-frequency relationship of the recorded waveform due to $20 \mathrm{kips}$ tensile force. The $1 / 2$-inch seven-wire strand Grade 250 is used. 


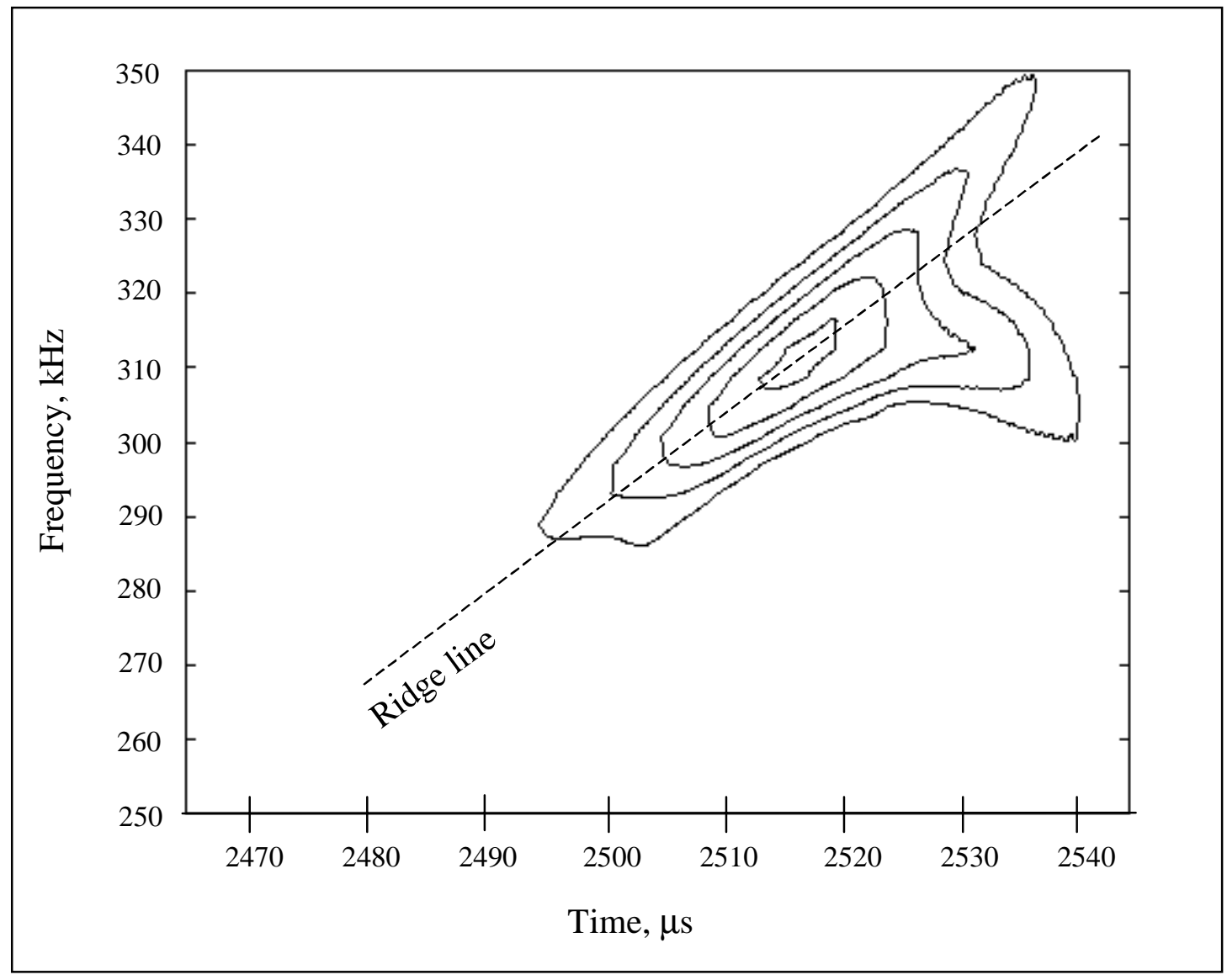

Figure 5.6 Time-frequency relationship of the recorded waveform due to $25 \mathrm{kips}$ tensile force. The $1 / 2$-inch seven-wire strand Grade 250 is used. 


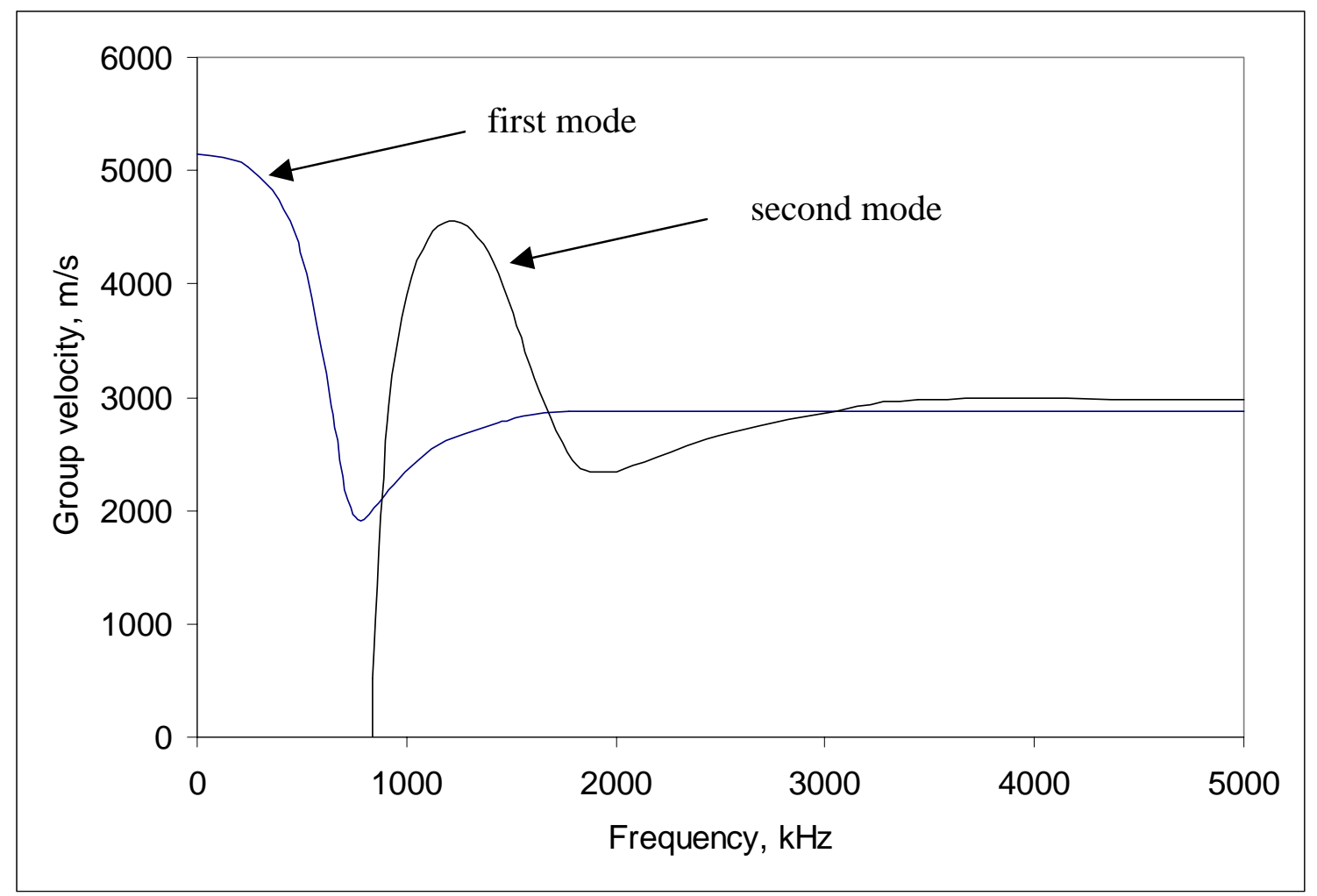

Figure 5.7 Group velocity of the first two longitudinal modes for the center wire $(\varnothing=$ $4.2 \mathrm{~mm})$. There is no tensile force applied. 


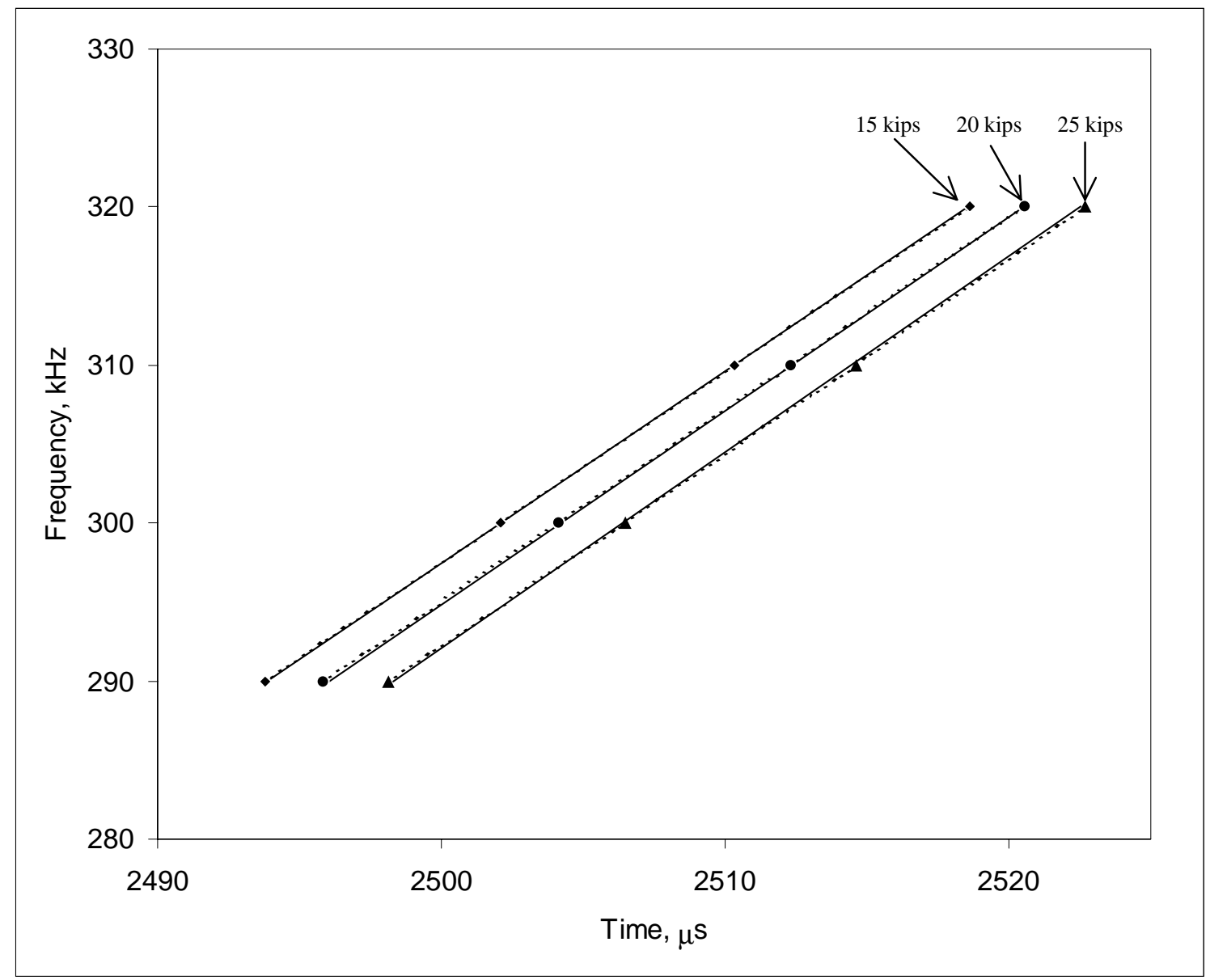

Figure 5.8 Time-frequency relationships of waveforms due to $15 \mathrm{kips}, 20 \mathrm{kips}$, and 25 kips tensile forces. Dotted lines represent experimental results. Solid lines represent numerical results. The $1 / 2$ inch seven-wire strand Grade 250 is used. 


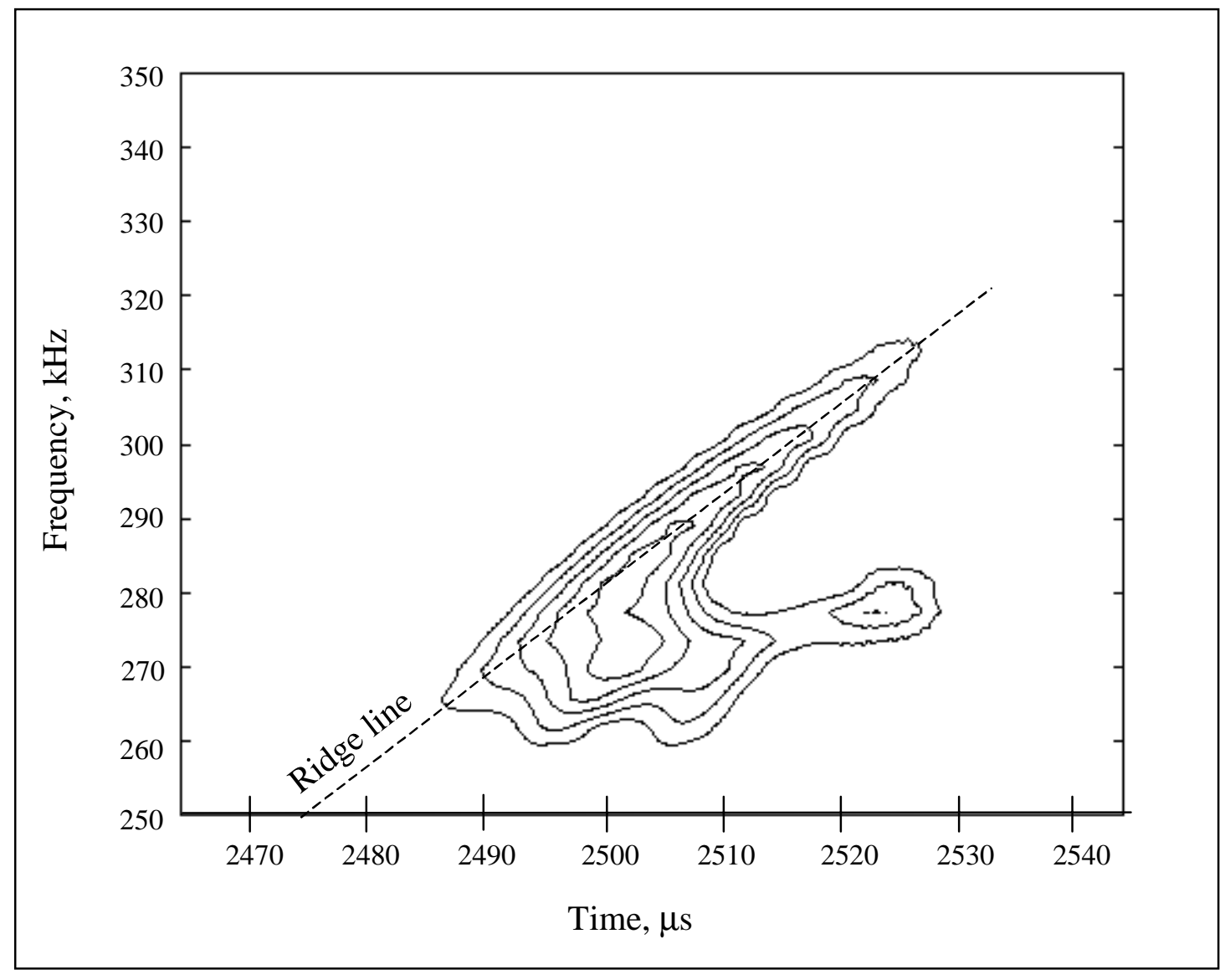

Figure 5.9 Time-frequency relationship of the recorded waveform due to 12 kips tensile force. The $1 / 2$-inch seven-wire strand Grade 270 is used. 


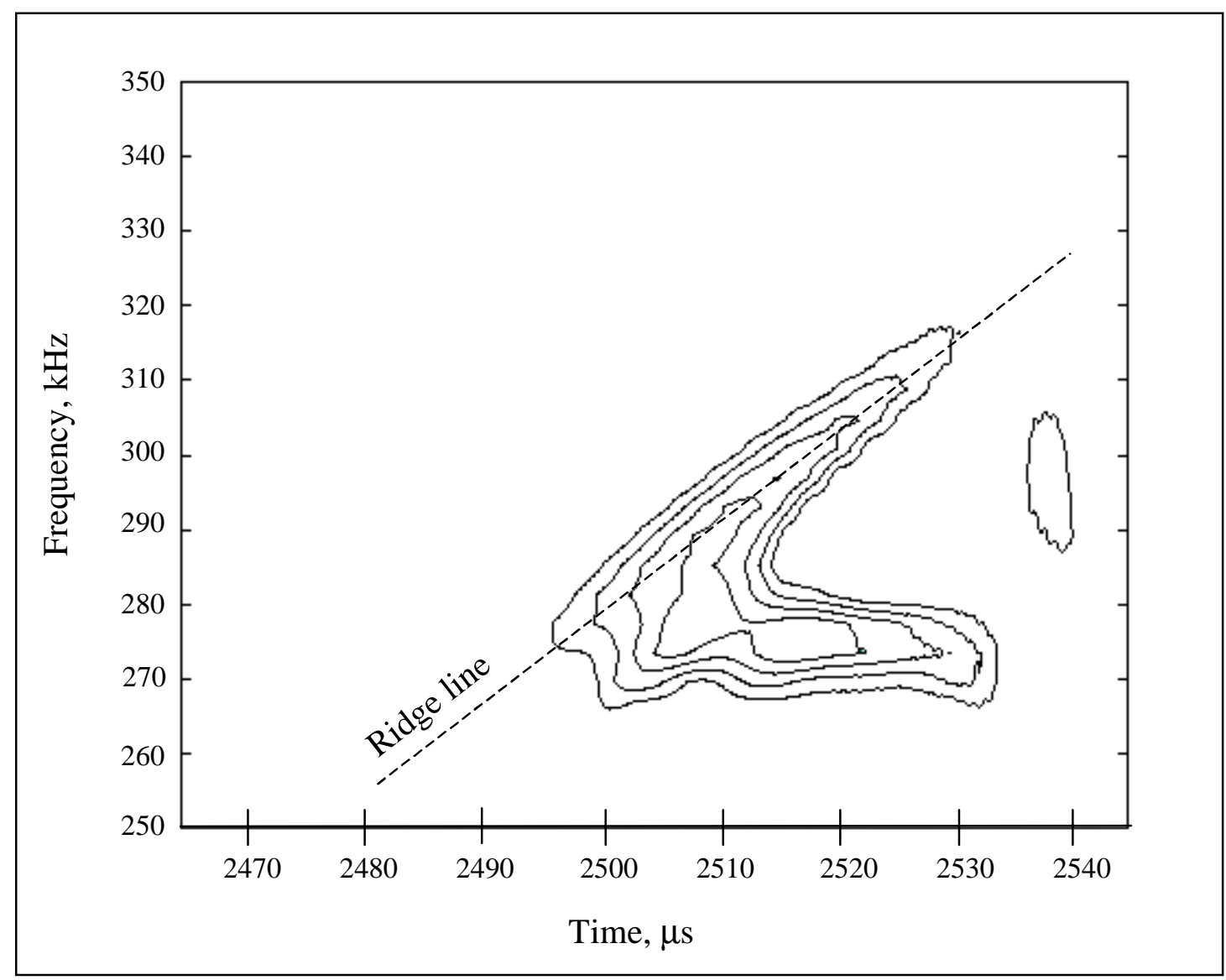

Figure 5.10 Time-frequency relationship of the recorded waveform due to $16 \mathrm{kips}$ tensile force. The 1/2-inch seven-wire strand Grade 270 is used. 


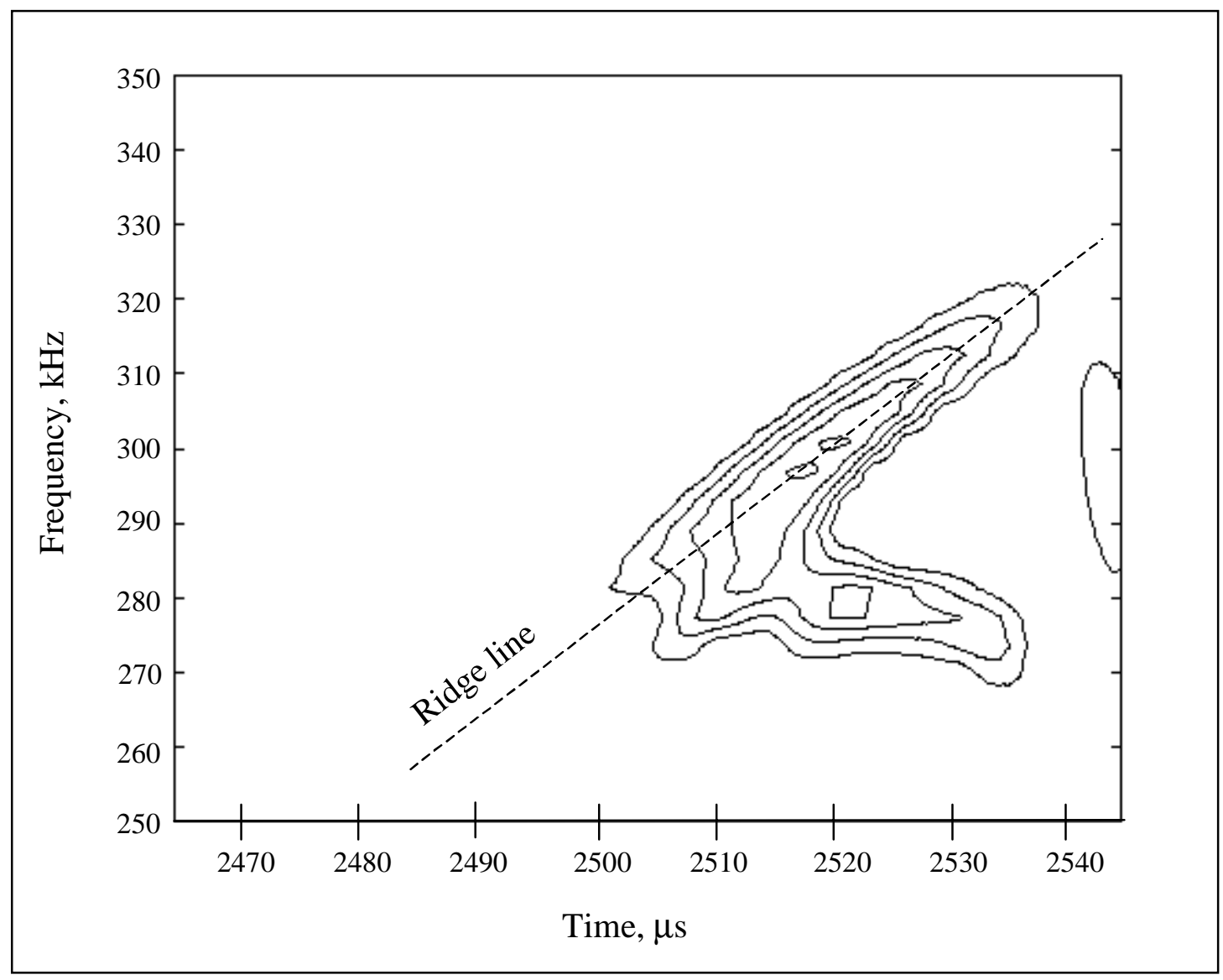

Figure 5.11 Time-frequency relationship of the recorded waveform due to 20 kips tensile force. The $1 / 2$-inch seven-wire strand Grade 270 is used. 


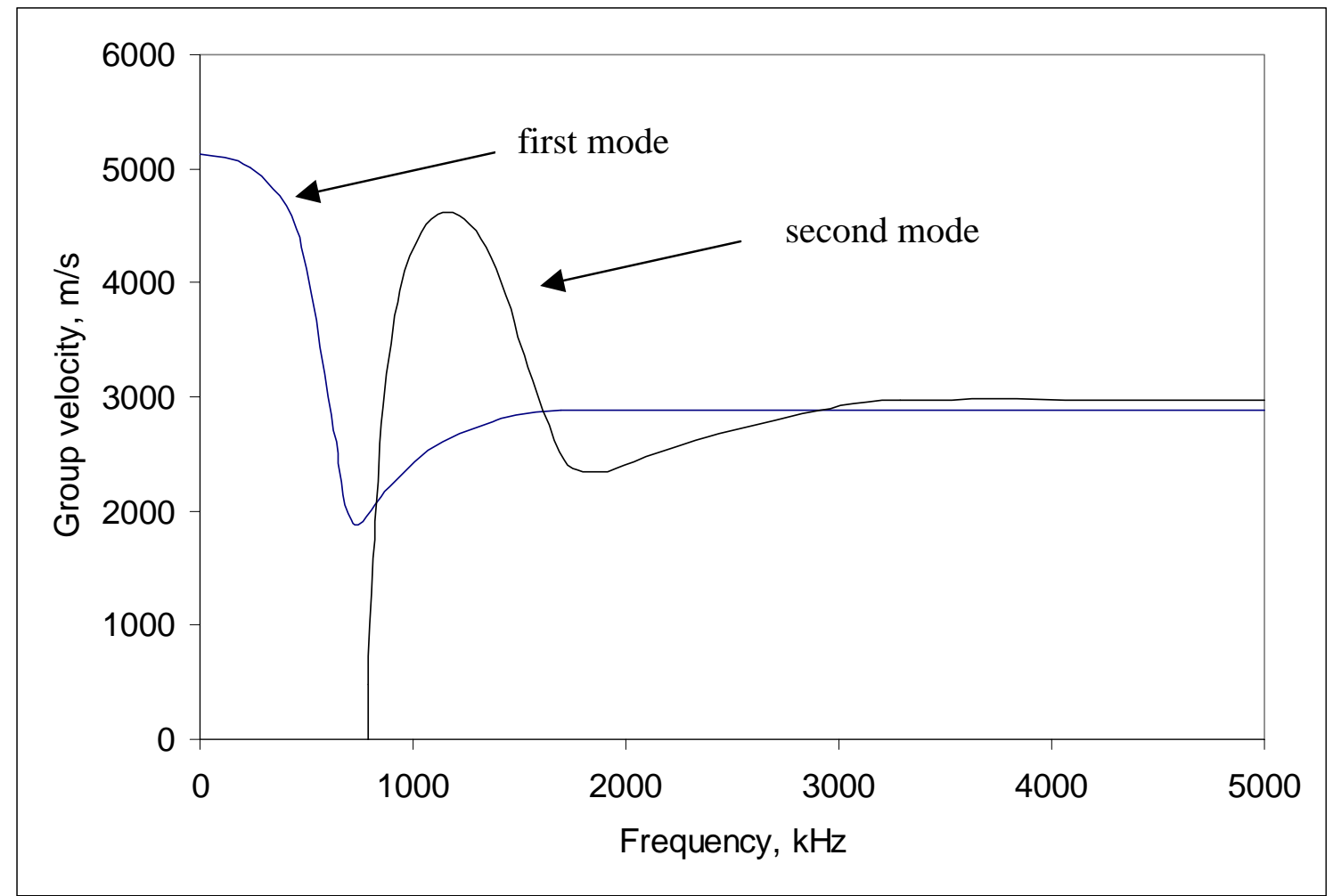

Figure 5.12 Group velocity of the first two longitudinal modes for the center wire $(\varnothing=$ $4.4 \mathrm{~mm}$ ). There is no tensile force applied. 


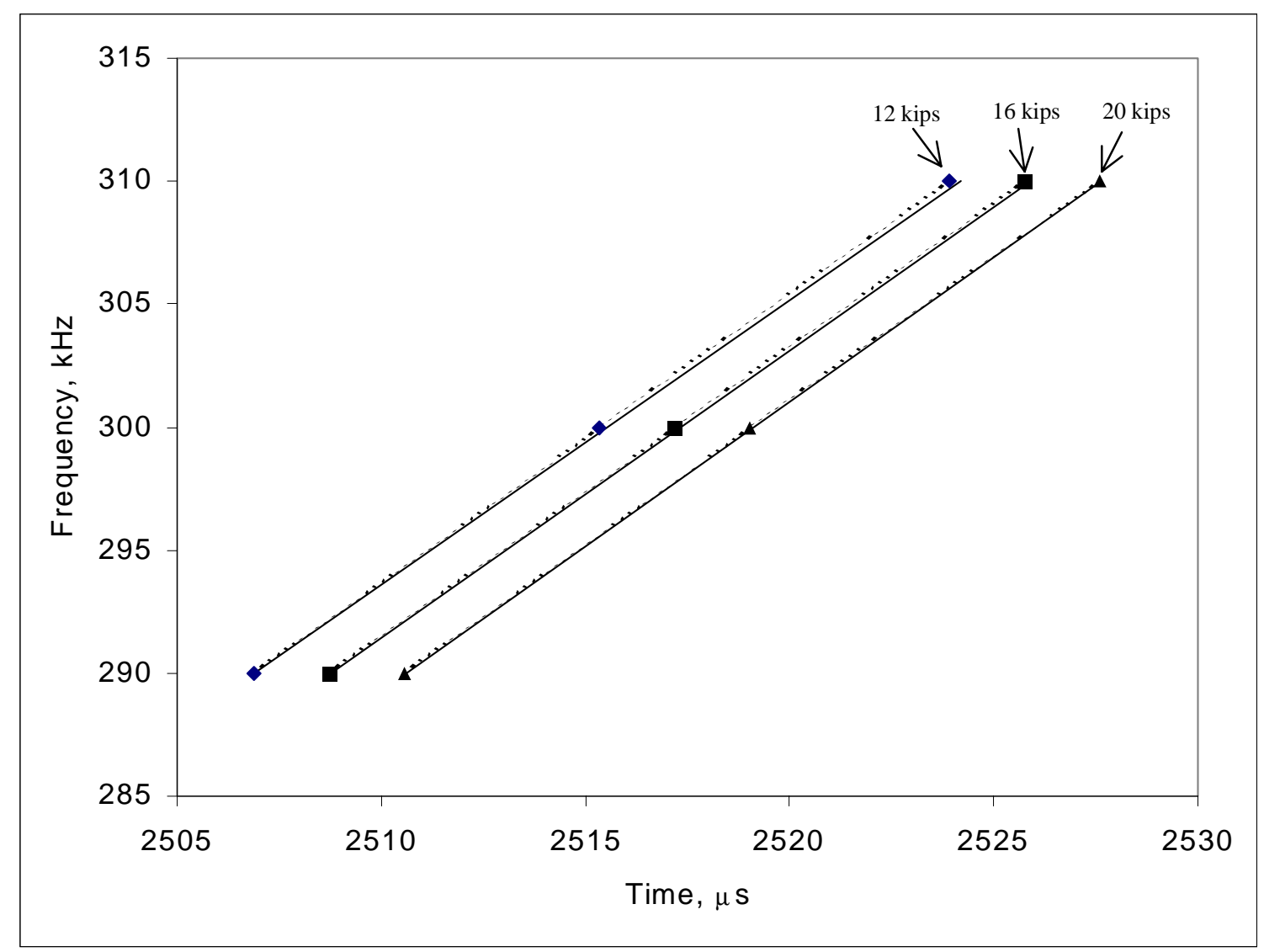

Figure 5.13 Time-frequency relationships of waveforms due to $12 \mathrm{kips}, 16 \mathrm{kips}$, and 20 kips tensile forces. Dotted lines represent experimental results. Solid lines represent numerical results. The $1 / 2$ inch seven-wire strand Grade 270 is used. 


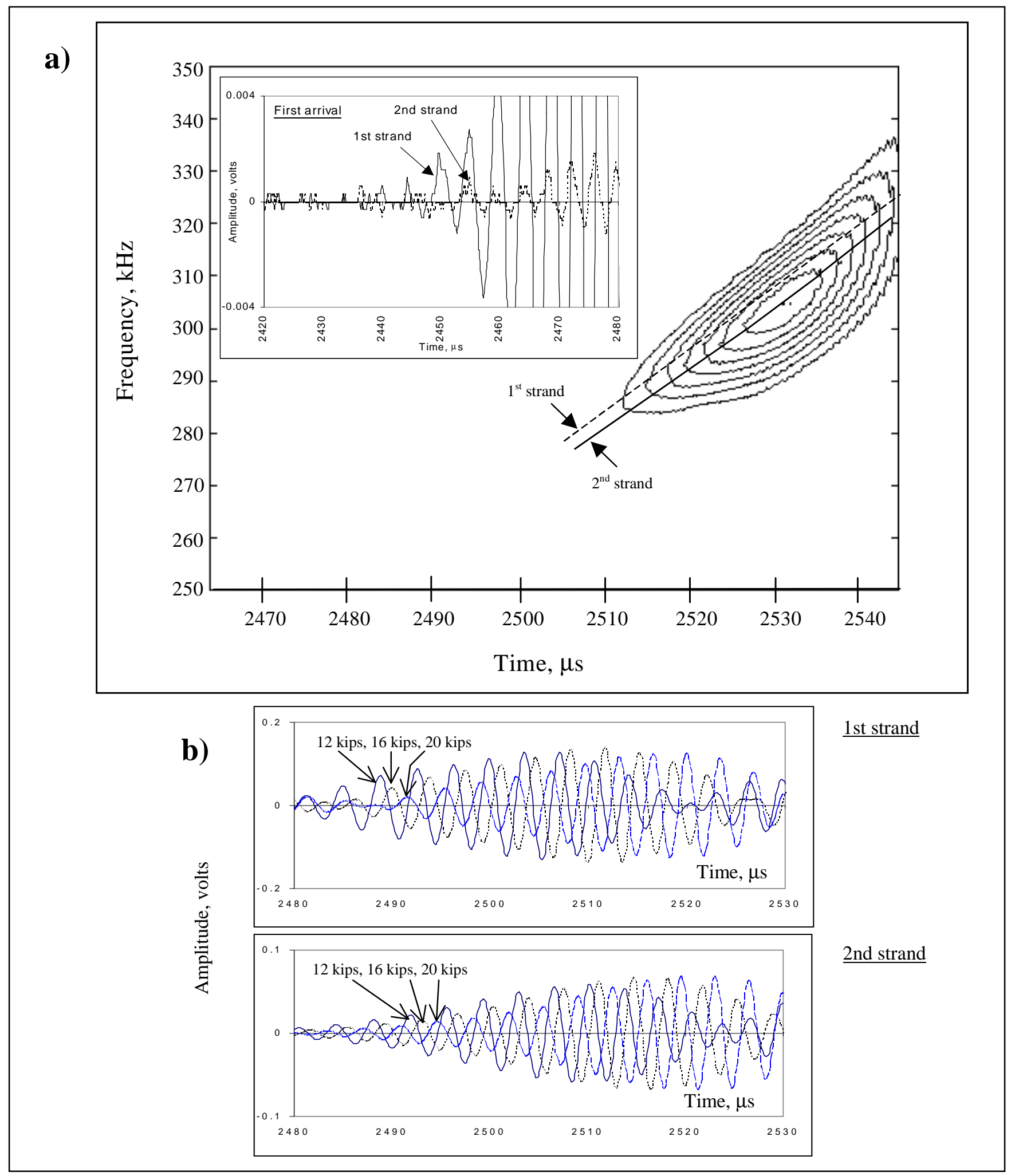

Figure 5.14 (a) Comparison between time-frequency relationships of two different strands subjected to 28 kips tensile force. The dotted line represents the numerical results of the 1st strand. The solid line represents the ridge line of the 2 nd strand. (b) time shift comparison between waveforms detected from these two strands. 


\section{CHAPTER 6}

\section{FIELD MEASUREMENTS OF TENSILE FORCES IN SEVEN-WIRE PRESTRESSING STRANDS}

\subsection{OVERVIEW}

The main objective of the study in this chapter is to demonstrate the use of the developed stress wave technique in evaluating prestress forces of the strands in actual posttensioned and pretensioned concrete structures. In this study, experiments were conducted at two prestressed concrete manufacturers. A total of four $1 / 2$-inch diameter seven-wire prestressing strands ( 2 pretensioning strands and 2 post-tensioning strands) were tested in these experiments. Based on theoretical studies described in the previous chapters, numerical analyses were conducted by taking into account of the acoustoelasticity effect and stress wave dispersion phenomenon in order to calculate the prestress levels in the strands. The Wigner-Ville transform technique was employed for identifying the arrival time of each frequency component of the stress wave signals. Change of the stress wave velocity at different stress levels was evaluated and the accuracy of this technique is discussed. In conjunction with the field experiments, a laboratory experiment was conducted in order to investigate the attenuation of a stress wave propagating in the strands used in pre-tensioning and post-tensioning systems. As a result of this experiment, the monitoring range of this technique is determined. 


\subsection{POST-TENSIONING STRANDS}

\subsubsection{Marietta Experiment}

$\underline{\text { Experimental Setup and Testing Procedures }}$

The first experiment for post-tensioning system was conducted at Marietta Structures Corp., a prestressed concrete manufacturer located in Marietta, OH. A schematic drawing of the experimental setup is shown in Figure 6.1. The casting bed was designed to manufacture four pre-tensioning concrete box girders simultaneously. One of the $1 / 2$-inch diameter sevenwire low-relaxation strands (Grade 270) was used as a test specimen. The strand is $83.94 \mathrm{~m}$ $(275.4 \mathrm{ft})$ long and the actual length under tension is $83.50 \mathrm{~m}(274.0 \mathrm{ft})$. Tensile force was gradually applied to the strand by a hydraulic jack. The maximum applied tensile force was 32 kips. A steel chuck was used at each end. The entire experiment was conducted before the concrete was poured into the casting bed so that there was no direct contact between the strand and concrete.

At one end of the strand (Figure 6.2), an Acoustic Emission (AE) transducer (PICO sensor, PAC) and a pulse generator (C-101-HV, PAC) were used to generate a stress wave impulse. At the other end of the strand (Figure 6.3 and Figure 6.4), another AE transducer, which is sensitive in a frequency range of $100 \mathrm{kHz}$ to $1 \mathrm{MHz}$, was used to capture the wave motions in the longitudinal direction. Both transducers were carefully mounted as perpendicular to the wire's cross section as possible to measure the longitudinal wave motions. The diameter of the AE transducers was about the same as the diameter of the 
center wire so that there was no contact between the transducers and the helical wires. A silicone grease compound (Ultragel II) was used as a coupling agent.

Experimental procedures were similar to the ones described in chapter 5. The strand was tested at different tension levels. Wave motions were generated by the pulse generator and were detected by the output transducer. They were then recorded by the MISTRAS system. All signals were digitized at $2 \mathrm{MHz}$ sampling rate. Multiple sets of data were recorded at each tension level to ensure the reproducibility of the results. After each data set was recorded, the strand was gradually loaded to a new tension level and both transducers were checked to ensure a proper contact between the transducers and the wire prior to the recording of a new data set.

\section{$\underline{\text { Results and Discussions }}$}

Figure 6.5 shows a typical arrival waveform recorded from the experiment. The strand was subjected to 20 kips tensile force. The triggering signal from the pulse generator, as a reference point in time, is also shown in this figure. By applying the Fourier transform technique, frequency components constituted in this waveform are shown in Figure 6.6. Results show that the frequency components of the waveform constitute mainly from 300 $\mathrm{kHz}$ to $400 \mathrm{kHz}$. It is also found that the frequency components are similar at all tension levels.

Results of the WVT, which present the time-frequency relationship of the recorded waveforms, are shown in Figure 6.7 - Figure 6.9. In these figures, three waveforms subjected to different tensile forces of 20 kips, 26 kips, and 32 kips were analyzed. WVT results clearly illustrate the dispersive behavior of the waves within the $300 \mathrm{kHz}-400 \mathrm{kHz}$ 
range of the first longitudinal mode. Hence, the lower frequency components would arrive at the receiving end earlier than the higher frequency components. Also shown in these figures, amplitudes of the frequency components are represented by the closed island shape contours, which have their peaks at about $350 \mathrm{kHz}$ (e.g. Figure 6.9). According to our assumption, the waveform would constitute only one frequency component at each particular point of time. However, the WVT results show a narrow band of frequencies throughout the monitoring time period. The arrival time of each frequency component of the waveform was then approximated by the ridge line of each WVT contour.

After the arrival times of all frequency components of the detected waveforms due to different tensile forces have been identified, material constants of the strand (Table 6.1) were determined by the trial-and-error method. Each individual constant was adjusted according to its influence on the group velocity (Table 6.2). Numerical analysis based on the frequency equation was conducted to produce the dispersion curve of the first longitudinal mode. Figure 6.10 shows dispersion curves of a stress wave propagating in the tested strand. Experimental results confirm that frequency components of all recorded waveforms are well within the frequency range of the first longitudinal mode.

Assuming the material properties and strand length are given, an iteration procedure for estimating the tensile force of a strand can be summarized as: (1) analyze the recorded waveforms using WVT, (2) select an appropriate frequency ( $\omega)$ and identify its traveling time $(t)$ from WVT plot, (3) assume tensile force $(N)$ and calculate tensile stress $(\sigma)$ in the center wire, (4) use the frequency equation to calculate group velocity $\left(C_{g}\right)$ corresponding to $\omega$ and $\sigma,(5)$ calculate the arrival time $t_{1}$ of the particular $\omega$ using equation (4.9), and (6) if $t_{1}$ $=t$, then $N$ is the actual tensile force, else go to step (3) and reassume $N$ value. 
Figure 6.11 shows the calculated and the measured arrival times of each frequency component of the waveforms due to three different tension levels. The dotted lines represent the experimental results (ridge lines from WVT plots) due to $20 \mathrm{kips}, 26 \mathrm{kips}$, and 32 kips tensile forces. During the trial-and-error process, material constants were initially assumed and were adjusted to provide the best fit to the ridge lines of at least two tension levels (20 kips and 32 kips in this case). This is to take into account of the dispersive behavior of the traveling wave for each tension level and, at the same time, to take into account of the acoustoelastic effect due to changes in tensile forces. Based on the material constants listed in Table 6.1, the calculated time-frequency relationships of the first longitudinal mode are represented by solid lines and agree well with the experimental data (dotted line) of 20 kips (on the left) and 32 kips (on the right) in this figure. This figure clearly shows a forward time shift of each frequency component when the tensile force is increasing. The average forward time shift is $6 \mu$ s per $1 \mathrm{kip}$ increment of the tensile force. The accuracy of the tensile force prediction method is demonstrated by using the material constants to predict the timefrequency relationship due to a third tensile force (26 kips), which is represented by a solid line in the middle of Figure 6.11. Accurate prediction results at this tension level are obtained where the average prediction error is about $2.0 \mu \mathrm{s}$. Since the average forward time shift is $6 \mu$ s per 1 kip tensile force increment, the prediction error of all three tension levels is about $2.6 \%$ of the applied forces. This technique has been shown to work well for sevenwire prestressing strands with tensile stress between $18 \%$ and $77 \%$ of the ultimate strength of the strand.

The WVT results show that many frequency components, ranging from $320 \mathrm{kHz}$ to $360 \mathrm{kHz}$, constitute in the detected waveforms. Only one frequency is needed for tensile 
force monitoring when applying this technique on an existing prestressed concrete structure. As shown in Figure 6.12, arrival times of three frequency components due to different tension levels are plotted. The symbols represent the experimental results obtained using the WVT technique. The solid lines represent analytical results based on the calculated material constants. The experimental results have good agreement with the analytical results. By using this figure, the arrival time of one particular frequency component can be directly translated into the tension level in the strand. Once the figure is established for a particular strand, the tensile force can be easily estimated.

In order to ensure the reproducibility of the experimental results, multiple sets of experimental data were recorded at each tension level. Figure 6.13 shows a comparison between the WVT results generated from two recorded waveforms due to 32 kips tensile force. The results show only small differences in time and frequency relationship between the two waveforms. It was found that the measured arrival time error is less than $2.0 \mu \mathrm{s}$. Thus, the corresponding error in tensile force prediction is less than about $0.33 \mathrm{kip}$, or about $2.2 \mathrm{ksi}$, which is approximately 0.8 percent of the ultimate strength of the strand (270 ksi). The error among these experimental results may be due to boundary condition changes at interfaces such as between the chucks and the strand, or between the transducers and the center wire. Nevertheless, at each tension level, the experimental results are clear and reproducible.

It is noted that the sampling rate of $2 \mathrm{MHz}$, providing a time measurement resolution of $0.5 \mu \mathrm{s}$, is sufficient for recording the arrival parts of the waveforms. From the average time shift rate (6 $\mu$ s per $1 \mathrm{kip}$ ) obtained earlier, the resolution of one data point per 0.08 kip was received. 


\subsubsection{Winchester Experiment}

\section{Experimental Setup and Testing Procedures}

The second experiment for post-tensioning system was conducted at Shockey Bros., Inc., a prestressed concrete manufacturer located in Winchester, VA. The test structure (Figure 6.14) was one of the prestressed concrete girders manufactured for a football stadium parking structure at James Madison University. The girder was initially pretensioned with twenty-six 9/16-in diameter bonded seven-wire strands of Grade 270 and was additionally post-tensioned with thirty-two $1 / 2$-inch diameter unbonded draped seven-wire monostrands of Grade 270. The cross-section of the girder is shown in Figure 6.15. For post-tensioning system, one of the $1 / 2$-inch diameter strands was used as a test specimen. The strand is $15.288 \mathrm{~m}(50.16 \mathrm{ft})$ long and the actual length under tension is $14.58(47.83 \mathrm{ft})$. The monostrand system was used. In this system, all strands were coated and sheathed by a high-speed extrusion process. This operation involves the pressurized application of a corrosion-inhibited lubricant, followed by the application of a seamless plastic sheath (protection sleeve). This provides the relative low tendon friction and a double layer of corrosion protection. The anchorage setup for the monostrand system is shown in Figure 6.16. In this experiment, tensile force was gradually applied to the strand by a hydraulic jack. The maximum applied tensile force was 30 kips. The entire experiment was conducted one day after concrete pouring.

By using the same setup as previous experiments, the pulse generator was used to generate the stress wave impulses through the input transducer mounted at the sending end of the strand (Figure 6.17). At the receiving end of the strand (Figure 6.18), the output 
transducer was used to capture the wave motions in the longitudinal direction. Both transducers were carefully mounted perpendicularly to the wire's cross section. Ultragel II was used as a coupling agent. Due to the restricted boundary condition of the strand that was coated by the lubricant and the protecting sheath, it is possible that a stress wave propagating in the strand would be attenuated at a faster rate than the one traveling in a strand with a free boundary condition. Therefore, the impact hammer was also used as an alternative method for generating stress wave impulses. The main difference between using the impact method (using impact hammer) and ultrasonic method (using pulse generator) is the frequency of stress waves in the strands. The pulse generator produces stress waves of relatively high frequency (short wavelength), whereas the impact hammer produces stress waves of low frequency (longer wavelength). The waves with lower frequency are associated with lower attenuation and thus propagate a longer distance. The experimental setup of this method is shown in Figure 6.19. As seen in Figure 6.20, each stress wave impulse was generated by pounding perpendicularly to the cross-section of the strand. The experimental procedures were similar to those in previous experiments. The MISTRAS system was used to record wave motions at the receiving end using $8 \mathrm{MHz}$ sampling rate. Multiple sets of data were recorded at each tension level to ensure the reproducibility of the results.

\section{$\underline{\text { Results and Discussions }}$}

The experiment was first conducted using the pulse generator to produce stress wave impulses at the sending end of the strand; however, there was no stress wave signal detected at the receiving end. The result shows that the entire energy of the waves was diminished 
before they could propagate the full length of the strand. Attenuation of the wave energy was primarily due to the lubricant and the protection sleeve.

The experiment was then proceeded by using the impact hammer as an alternative way of sending stress wave impulses into the strand. By using impact excitations, the output transducer was able to detect the incoming signal at the receiving end. Figure 6.21 shows a typical arrival waveform recorded from the experiment. The strand was subjected to 10 kips tensile force. The triggering signal from the impact hammer is also shown in this figure. By applying the Fourier transform technique, frequency components constituted in this waveform are shown in Figure 6.22. The result shows that the recorded waveform contains frequency components within a narrow range between $14 \mathrm{kHz}$ and $16 \mathrm{kHz}$. The frequencies in this range were also constituted in the waveform recorded at all tension levels. As shown in Figure 6.23, the corresponding result was obtained by applying WVT to this waveform. From this figure, it is very difficult to identify the arrival time of any particular frequency directly from the WVT plot because the dispersive behavior of the wave was not clearly illustrated. This is because frequencies of the detected waveforms are in very narrow range approaching the bar velocity so that there were very small differences of group velocities (or arrival times) among these frequencies.

For this reason, analysis of the experimental results has to be focused only on identifying and comparing the arrivals of the waveforms' first cycles due to different tensile forces. The first cycle of each waveform has its amplitude just above the average background noise. Figure 6.24 shows the first arrivals of three waveforms due to 5 kips, 10 kips, and 15 kips tensile forces. It is shown that there are large differences in shape and amplitude among these waveforms. In this case, the same cycles among these waveforms 
cannot be matched. This is the reason why time shift of the latter cycles (as described in chapter 4) cannot be measured. By comparing the first arrivals of these waveforms, the result shows that there is a forward time shift of the waveform when higher tensile force is applied to the strand. The plot between arrival times of the first cycles and applied tensile forces is shown in Figure 6.25. After conducting a regression analysis, the trend line drawn in this figure designates the best fit to the experimental results. It is approximated that the forward time shift is $1.46 \mu$ s for each 1 kips increment of the tensile force. The strand's material constants (Table 6.3) were determined by using the trial-and-error method in order to adjust the material constants to fit the experimental results in Figure 6.25. The approximated single frequency of $15 \mathrm{kHz}$ was assumed in the analysis. It is worthwhile to mention that the arrival time of only one frequency component was considered in this case. Therefore, the calculated material properties may not be as accurate as those obtained in Table 6.1 where arrival times of many frequency components were considered in numerical calculations. Based on these material constants, dispersion curves of a stress wave propagating in the center wire are shown in Figure 6.26. It can be seen from this figure that the approximated frequency is relatively close to $0 \mathrm{~Hz}$ on the dispersion curve of the first longitudinal mode, which is almost flat in the low-frequency region. Therefore, there are no significant differences of the group velocities among the frequencies within this range. This statement was verified by Table 6.4 where calculated group velocities and arrival times among frequency components of $0 \mathrm{~Hz}, 14 \mathrm{kHz}, 15 \mathrm{kHz}$, and $16 \mathrm{kHz}$ are compared. For example, when the strand is subjected to 30 kips tensile force, the calculated traveling time of $14 \mathrm{kHz}$ component is $3005.96 \mu$ s and the traveling time of $16 \mathrm{kHz}$ component is $3006.02 \mu \mathrm{s}$. Therefore, the traveling time difference between these two frequencies is $0.06 \mu$ s and is 
considered very small. Correspondingly, the variation of the calculated tensile force between $14 \mathrm{kHz}$ and $16 \mathrm{kHz}$ is \pm 0.04 kip.

It was observed from multiple set of experimental data that the differences of arrival times among waveforms recorded at each tension level are less than $2 \mu$ s. Thus, the corresponding error in tensile force prediction is less than $1.37 \mathrm{kip}$, or about $8.95 \mathrm{ksi}$, which is approximately 3.3 percent of the maximum strength of the strand $(270 \mathrm{ksi}) . \quad$ The error among these results is due to the fact that the stress wave impulses generated from different events are not perfectly identical, especially in amplitudes, impact duration, and location of impact. In addition, the relatively low frequency (e.g. $15 \mathrm{kHz}$ ) also results in lower resolution. It was noticed that the noise level heavily affects accuracy of this method, especially when attempting to locating the first arrivals. Increasing the threshold level in the MISTRAS system will not only filter out the noise, but also the early parts of the waveform, which normally have relatively low amplitude. Therefore, the impact method may not be a reliable method for accurate measurement of tensile force in the strands.

\subsection{PRETENSIONING STRANDS}

\subsubsection{Marietta Experiment}

Two field experiments were conducted in order to investigate the stress wave propagation in the seven-wire prestressing strands used in pretensioned concrete structures. The first experiment was conducted on the existing pretensioned concrete box girder provided by Marietta Structures Corp. (Figure 6.27). A schematic drawing of the experimental setup is shown in Figure 6.28. Three 1/2-inch diameter seven-wire stress- 
relieved strands (Grade 270) were tested. The actual length under tension for all of the strands is $13.69 \mathrm{~m}(44.92 \mathrm{ft})$. During the manufacturing process, tensile force of 32 kips was initially applied to each strand. The strands were cut and the girder was taken out of the casting bed one day after concrete pouring. The entire experiment was also conducted on this day. The compressive strength of the concrete between 4000 psi and 7000 psi was expected.

At one end of each strand, an input transducer and the pulse generator was used to generate a stress wave impulse. At the other end of the strand, an output transducer was used to capture the wave motions in the longitudinal direction. Both transducers were carefully mounted perpendicular to the wire's cross section to measure the longitudinal wave motions. Ultragel II was used as a coupling agent.

The experiment was conducted repeatedly for each of the three strands; however, there was no stress wave signal detected at the receiving end. This is possibly because the entire wave energy was absorbed by concrete, which is a highly attenuative material. It can be expected that a longitudinal stress wave would undergo an attenuation of its amplitude as it travels along the length of the strand. Experimental study of this phenomenon is described in the next section.

\subsubsection{Winchester Experiment}

The second experiment was conducted at Shockey Bros., Inc. The test specimen was the same prestressed concrete girder that was previously tested using a post-tensioning strand (Figure 6.14). One of the twenty-six 9/16-inch diameter seven-wire pretensioned strands was tested. The strand is $14.66 \mathrm{~m}(48.10 \mathrm{ft})$ long and the actual length under tension is 
$14.38 \mathrm{~m}(47.17 \mathrm{ft})$. The tensile force of 36.3 kips was initially applied to the strand. The entire experiment was conducted one day after the casting of concrete when the concrete's compressive strength of 4000 psi was attained. On this day, the jacking force was released, the concrete girder was taken out of the casting bed, and the experiment began.

Experimental setup was arranged in the same way as previously shown in Figure 6.28. The pulse generator was used to generate the stress wave impulses through the input transducer mounted at the sending end of the strand. At the receiving end of the strand, the output transducer was used to capture the wave motions in the longitudinal direction.

The previous experimental result shows that the stress wave propagating along the pretensioned strand was severely attenuated. Therefore, the impact hammer was used as an alternative method for generating input signals. The similar experimental setup was prepared in the same way as shown in Figure 6.19.

Experiments were conducted repeatedly using the pulse generator; however, there was no stress wave signal detected at the receiving end. Nevertheless, there was still no signal detected at the receiving end when using impact excitations.

\subsubsection{Attenuation of Stress Waves in the Seven-Wire Strands Bonded with Concrete}

It has been observed from previous experimental results that all recorded stress waves from the center wire exhibited some reductions in amplitude when propagating in the strands for both post-tensioning and pretensioning cases. For post-tensioning systems, despite there was no contact between strands and concrete, attenuation of the peak amplitude still occurred. This is due to material attenuation of the steel strand itself and transmission of some stress wave energy from the center wire to the helical wires. For strands used in 
pretensioning systems, the attenuation is not only due to the above causes, but also due to the transmission of the stress wave energy from the strand to the surrounding concrete. Attenuation of the propagating stress waves is a major difficulty in this technique since the stress wave energy could be significantly reduced before reaching the receiving end of the strand.

The first investigation is focused on the attenatuaion rate of stress waves propagating in seven-wire post-tensioning strands. Experimental study and analysis were conducted based on results obtained from testing of $10 \mathrm{ft}$ and $71.2 \mathrm{ft}$ seven-wire prestressing strand and from results obtained from the previous experiments where two prestressing strands with the lengths of $40.52 \mathrm{ft}$ and $275.4 \mathrm{ft}$ were tested. From these results, similar frequency components between $300 \mathrm{kHz}$ and $400 \mathrm{kHz}$ were detected from all recorded waveforms. Figure 6.29 shows reductions of peak amplitude as the strand length increases. Attenuation of a stress wave propagating in the strand can be expressed as:

$$
A(x)=A_{o} e^{-k_{1} x}
$$

where, $A_{0}$ is the original peak amplitude of the wave at the sending end of the strand. $A(x)$ is the peak amplitude of the wave after propagating the distance $x$ along the strand. $k_{l}$ is the attenuation coefficient due to strand's material attenuation and due to transmission of the energy from the center wire to the helical wires. By performing a regression analysis of the peak amplitude data, an approximated $k_{l}$ value of 0.02 was obtained. Equation 6.1 can be used to evaluate the maximum monitoring range when strands of different lengths are tested. 
This can be used in selecting appropriate pulse generators for different situations so as to ensure that the waves can propagate through the entire length of the strand.

The second investigation is focused on the attenatuaion rate of the stress waves in pretensioning strands. In this case, there is a direct contact between the strands and concrete. Bonding between the two is gradually developed when concrete is hardening. The prestress force is transferred from the strands to the concrete through the contact area between them. Thus, some amount of the stress wave energy would transmit from the strand to concrete. Therefore, the monitoring range in the pretensioning system would be limited to a relatively short distance in the strand.

Figure 6.30 shows the experimental setup where a $10 \mathrm{ft}$ long seven-wire prestressing strand was embedded inside a concrete beam. The bond length between the strand and the concrete was varied from $0 \mathrm{ft}$ to $10 \mathrm{ft}$. The typical test setup was followed where the pulse generator was used to generate a short duration stress wave impulse through the input transducer. The output transducer was used to detect wave motions in the longitudinal direction. Ultragel II was used as a coupling agent. The detected wave motions were recorded by the MISTRAS system at each bond length. All signals were digitized at $4 \mathrm{MHz}$ sampling rate. There was no tensile force applied to the strand.

Recorded waveforms are presented in Figure 6.31. Results indicate that peak amplitude of the waveform is reduced as the bond length increases. The peak amplitude, $A(x)$, of the wave after propagating the distance $x$ along the strand bonded with concrete can be expressed as:

$$
A(x)=A_{o} e^{-k_{1} x} e^{-k_{2} x}
$$


where, $k_{2}$ is the attenuation coefficient due to the transmission of the stress wave energy from the strand to concrete. In this experiment, the strand length remains constant at $10 \mathrm{ft}$. Therefore, equation (6.2) can be written as:

$$
A(x)=C e^{-k_{2} x}
$$

where, $C$ is a constant. Figure 6.32 shows the decay of peak amplitude as the bond length increases. By performing the regression analysis, the approximated $k_{2}$ value of 0.26 was obtained. Results indicate that stress waves exhibits a very severe reduction in amplitude when propagaing farther along in the strand bonded with concrete. Using these attenuation coefficients, equation (6.2) can be written as:

$$
A(x)=A_{o} e^{-0.02 x} e^{-0.26 x}=A_{o} e^{-0.28 x}
$$

As far as the detectability of the signals is concerned, equation (6.1) and equation (6.4) can be used to estimate the monitoring range (the maximum strand length) of the strands for each type of prestressing systems. It is observed that an average noise level in our experiments is about $1 \times 10^{-3}$ volt. As far as signal resolution is concerned, the peak amplitude of detected waveforms should be considerably higher than the average noise level. For example, the signal-to-noise ratio of 10 or higher is recommended. Therefore, the desireable peak amplitude should be at least $1 \times 10^{-2}$ volt. By substituting $k_{l}$ value $(0.02)$ and desired $A(x)$ value ( $1 \times 10^{-2}$ volt) into equation (6.1), the monitoring range (x) of $295 \mathrm{ft}$ was 
obtained for post-tensioning strands. By substituting $k_{2}$ value $(0.28)$ and desired $A(x)$ value $\left(1 \times 10^{-2}\right.$ volt) into equation (6.4), the monitoring range (x) of $21 \mathrm{ft}$ was obtained for pretensioning strands. It is worthwhile to mention that the original peak amplitude $\left(\mathrm{A}_{0}\right)$ is dependent on several factors such as input energy (volts) from the pulse generator, operating range and sensitivity of the input transducer, contact conditions between the input transducer and the strand, and the type of coupling agents. In determining the monitoring range using different types of experimental setup and instrumentation, a preliminary test using a similar approach may be needed. 


\subsection{SUMMARY}

This chapter describes field measurements of the developed stress wave technique for estimating tensile forces in the seven-wire prestressing strands. Experiments were conducted on the strands used in both pretensioning and post-tensioning systems provided by two prestressed concrete manufacturers. This technique was shown to work well with the post-tensioning system where the stress wave impulses, generated by the pulse generator, were able to propagate a very long distance up to $275.4 \mathrm{ft}$. Satisfactory results were obtained from using the Wigner-Ville transform technique to analyze the recorded waveforms. The same stress wave impulses, however, could not propagate very far distance in the post-tensioning monostrand system due to the energy absorption by the coating lubricant and the protection sleeve. The impact hammer was used to generate low frequency impulses that could propagate a longer distance. Tensile force measurement was conducted by identifying the waveforms' first arrivals; however, these arrivals are difficult to identify due to the high noise level. For pre-tensioning system, attenuation of the waves are much more severe such that the stress wave impulses, generated by either pulse generator or impact hammer, could not propagate through the entire lengths of the strands $(44.92 \mathrm{ft}$ and $48.1 \mathrm{ft}$ ). By analyzing the data recorded from field and laboratory experiments, it is estimated that the monitoring range of this technique is limited to $21 \mathrm{ft}$ for pretensioning strands and $295 \mathrm{ft}$ for post-tensioning strands. 


\begin{tabular}{|c|c|c|c|}
\hline & \multicolumn{3}{|c|}{ Marietta Structures Corp. } \\
\hline$\rho$ & $=$ & $7.300 \times 10^{-4}$ & $\left(\mathrm{lb}-\mathrm{s}^{2} / \mathrm{in}^{4}\right)$ \\
\hline$\lambda$ & & $13.11 \times 10^{6}$ & (psi) \\
\hline$\mu$ & $=$ & $12.446 \times 10^{6}$ & (psi) \\
\hline 1 & $=$ & $-35.96 \times 10^{6}$ & (psi) \\
\hline $\mathrm{m}$ & $=$ & $-90.34 \times 10^{6}$ & (psi) \\
\hline $\mathrm{n}$ & & $-103.5 \times 10^{6}$ & (psi) \\
\hline
\end{tabular}

Where $\rho$ is the mass density; $\lambda$ and $\mu$ are Lame's elastic constants. $1, \mathrm{~m}$, and $\mathrm{n}$ are Murnaghan's elastic constants.

The nominal cross-sectional area of the strand is $0.153 \mathrm{in}^{2}\left(98.7 \mathrm{~mm}^{2}\right)$.

Table 6.1 Calculated material constants of a seven-wire prestressing strand (Grade 270) from the field measurement at Marietta Structures Corp. 


\begin{tabular}{|c|c|c|}
\hline $\begin{array}{c}\text { Material } \\
\text { Constant }\end{array}$ & $\begin{array}{c}\text { Group Velocity Variation } \\
(\mathrm{m} / \mathrm{s})\end{array}$ & $\begin{array}{c}\text { Tensile Force Variation } \\
\text { (kips) }\end{array}$ \\
\hline$\rho( \pm 1 \%)$ & $\mp 28.34$ & \pm 16.462 \\
$\lambda( \pm 1 \%)$ & \pm 0.62 & $\mp 0.357$ \\
$\mu( \pm 1 \%)$ & \pm 28.87 & $\mp 16.573$ \\
$1( \pm 1 \%)$ & $\mp 0.01$ & \pm 0.007 \\
$\mathrm{~m}( \pm 1 \%)$ & $\mp 0.74$ & \pm 0.430 \\
$\mathrm{n}( \pm 1 \%)$ & $\mp 0.21$ & \pm 0.122 \\
\hline
\end{tabular}

Table 6.2 Variations of group velocity and tensile force due to $\pm 1 \%$ change of the strand's material constants. (evaluated at propagating frequency $=350 \mathrm{kHz}$, tensile force $=32 \mathrm{kips}, \mathrm{C}_{\mathrm{g}}=4940 \mathrm{~m} / \mathrm{s}$ ). The $1 / 2$-inch seven-wire strand Grade 270 is used. 


\begin{tabular}{|c|c|c|c|}
\hline & \multicolumn{3}{|c|}{ Shockey Bros., Inc. } \\
\hline$\rho$ & $=$ & $7.300 \times 10^{-4}$ & $\left(\mathrm{lb}-\mathrm{s}^{2} / \mathrm{in}^{4}\right)$ \\
\hline$\lambda$ & & $13.110 \times 10^{6}$ & (psi) \\
\hline$\mu$ & $=$ & $11.947 \times 10^{6}$ & (psi) \\
\hline 1 & & $-35.96 \times 10^{6}$ & (psi) \\
\hline $\mathrm{m}$ & $=$ & $-94.95 \times 10^{6}$ & (psi) \\
\hline $\mathrm{n}$ & & $-83.50 \times 10^{6}$ & (psi) \\
\hline
\end{tabular}

Where $\rho$ is the mass density; $\lambda$ and $\mu$ are Lame's elastic constants. $1, \mathrm{~m}$, and $\mathrm{n}$ are Murnaghan's elastic constants.

The nominal cross-sectional area of the strand is $0.153 \mathrm{in}^{2}\left(98.7 \mathrm{~mm}^{2}\right)$.

Table 6.3 Calculated material constants of a seven-wire prestressing strand (Grade 270) from the field measurement at Shockey Bros., Inc. 


\begin{tabular}{|c|c|c|c|c|c|c|c|c|}
\hline \multirow{2}{*}{$\begin{array}{c}\text { Tensile } \\
\text { Force }\end{array}$} & \multicolumn{4}{|c|}{$\begin{array}{c}\text { Group Velocity, Cg } \\
(\mathrm{m} / \mathrm{s})\end{array}$} \\
\cline { 2 - 10 }$(\mathrm{kips})$ & $0 \mathrm{~Hz}$ & $14 \mathrm{kHz}$ & $15 \mathrm{kHz}$ & $16 \mathrm{kHz}$ & $0 \mathrm{~Hz}$ & $14 \mathrm{kHz}$ & $15 \mathrm{kHz}$ & $16 \mathrm{kHz}$ \\
\hline 0 & 5161.44 & 5161.03 & 5160.97 & 5160.91 & 2961.97 & 2962.20 & 2962.23 & 2962.27 \\
5 & 5154.81 & 5154.40 & 5154.35 & 5154.29 & 2969.07 & 2969.30 & 2969.33 & 2969.37 \\
10 & 5148.08 & 5147.68 & 5147.63 & 5147.57 & 2976.24 & 2976.47 & 2976.50 & 2976.54 \\
15 & 5141.25 & 5140.86 & 5140.81 & 5140.75 & 2983.50 & 2983.72 & 2983.75 & 2983.79 \\
20 & 5134.32 & 5133.94 & 5133.89 & 5133.83 & 2990.83 & 2991.05 & 2991.08 & 2991.12 \\
25 & 5127.29 & 5126.91 & 5126.86 & 5126.81 & 2998.24 & 2998.46 & 2998.49 & 2998.53 \\
30 & 5120.14 & 5119.77 & 5119.72 & 5119.67 & 3005.74 & 3005.96 & 3005.99 & 3006.02 \\
\hline
\end{tabular}

Table 6.4 Calculated group velocities and arrival times of several frequency components of the detected waveforms due to different tensile forces 


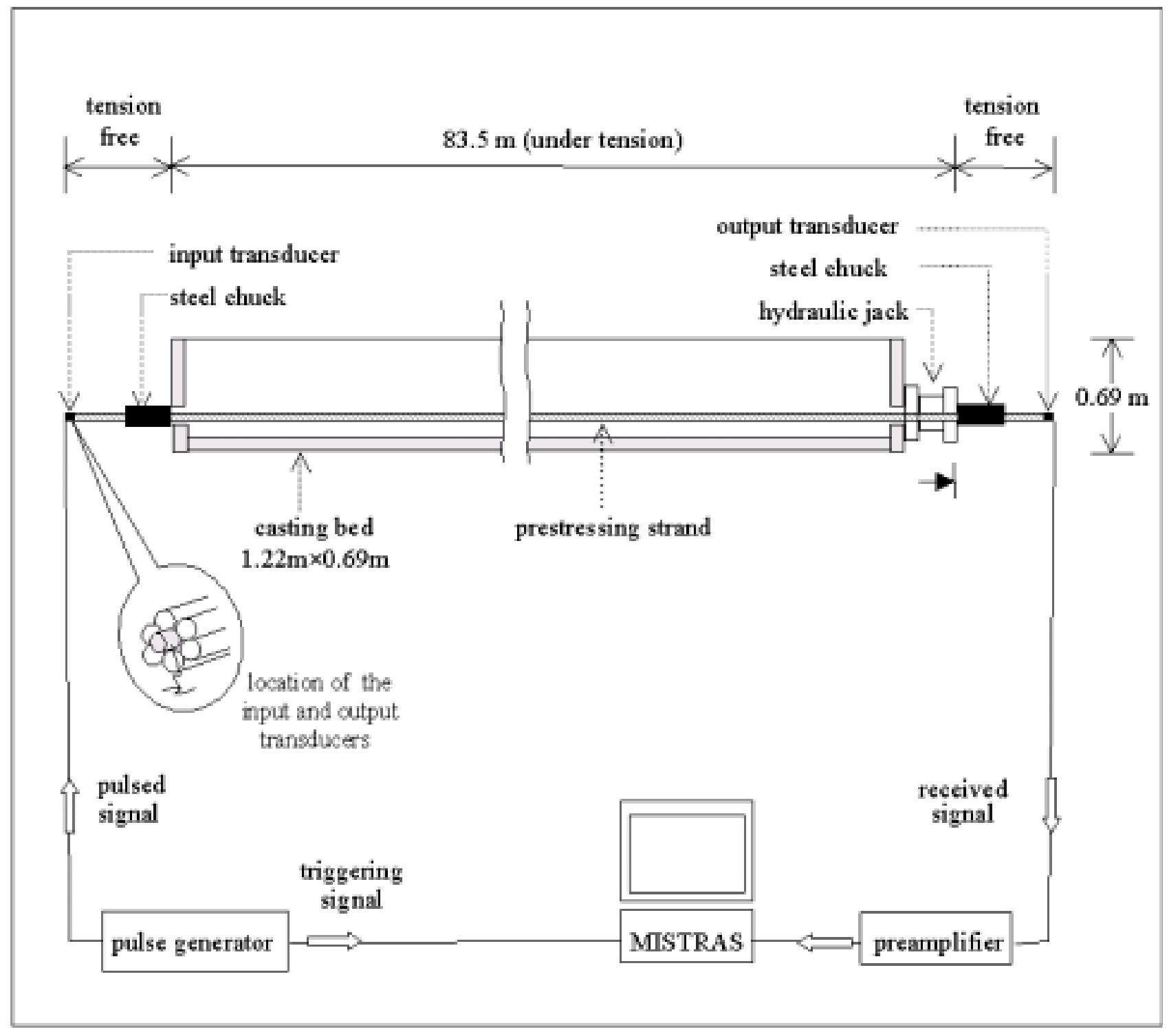

Figure 6.1 Experimental setup. 


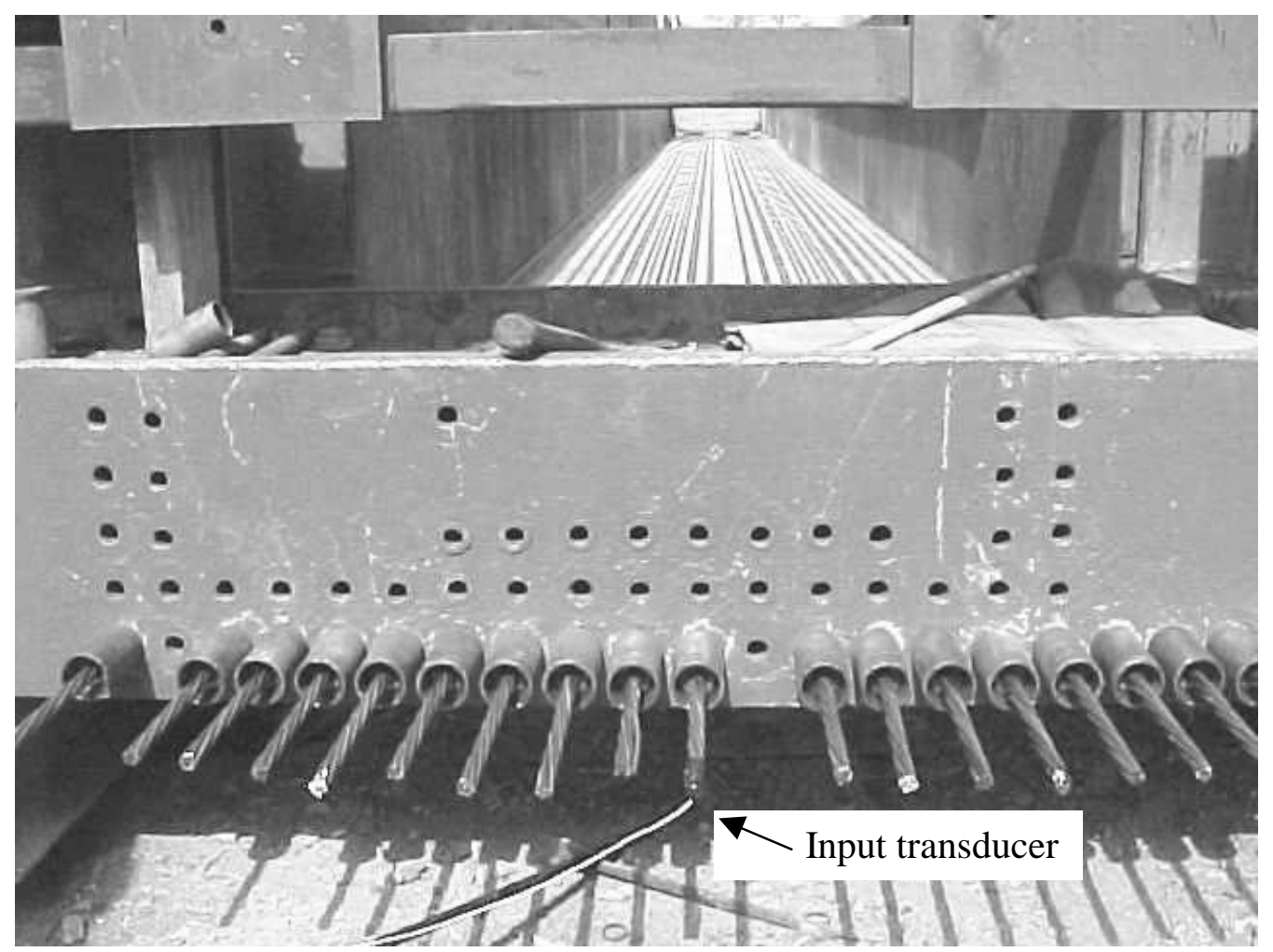

Figure 6.2 Pulsing end of the prestressing strand 


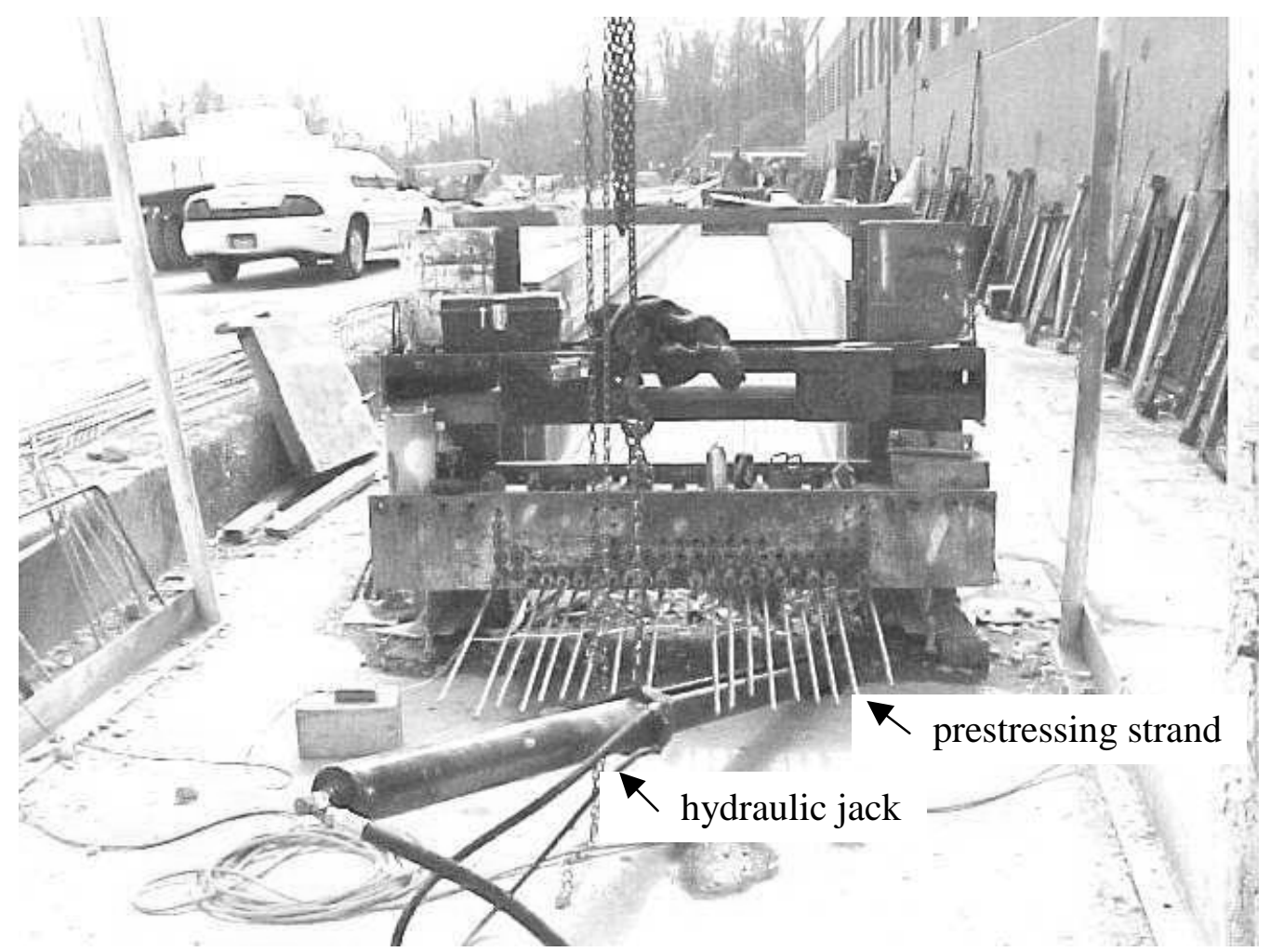

Figure 6.3 Receiving and loading end of the prestressing strand 


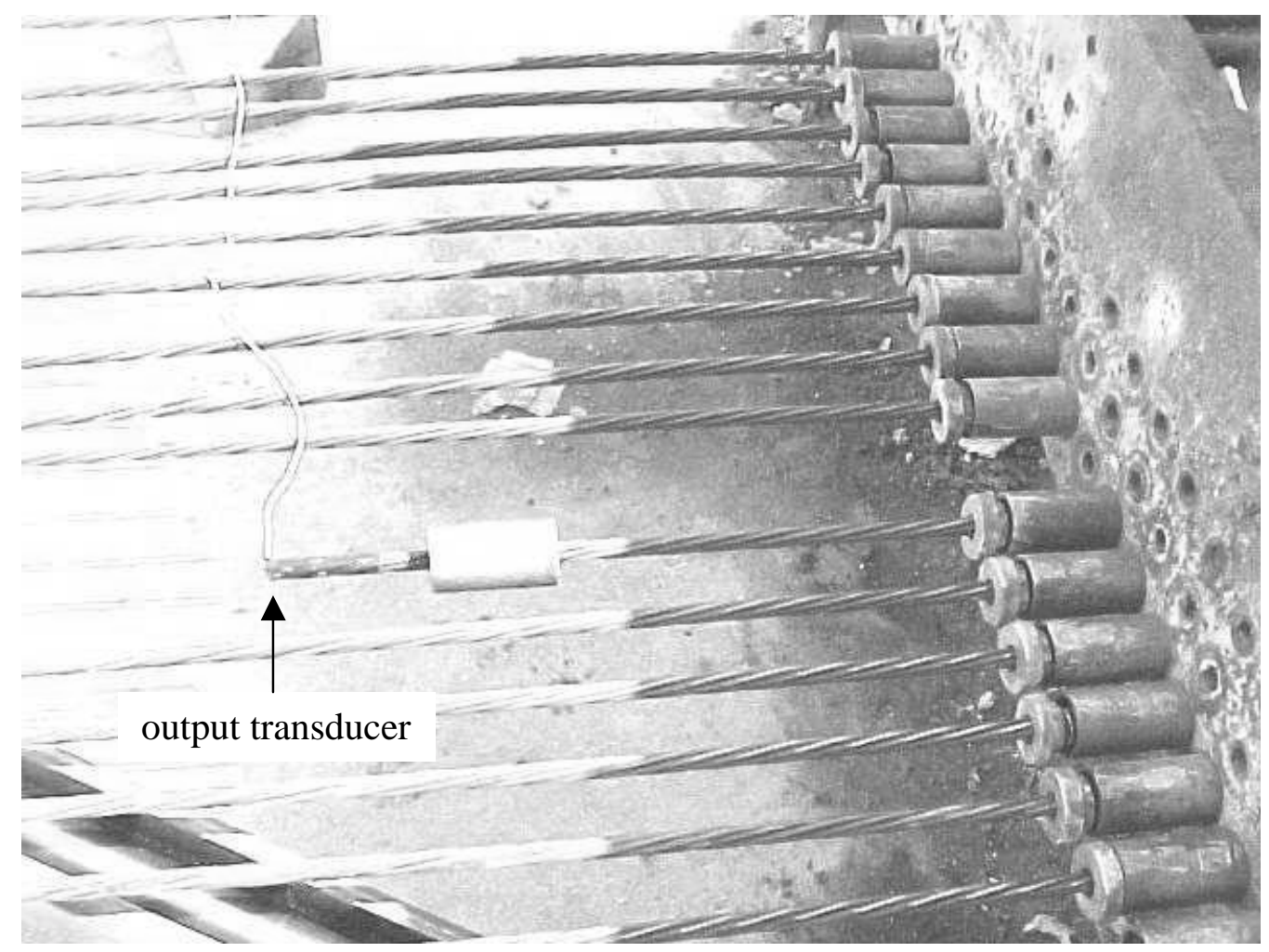

Figure 6.4 Connection between the output transducer and the seven-wire prestressing strand 


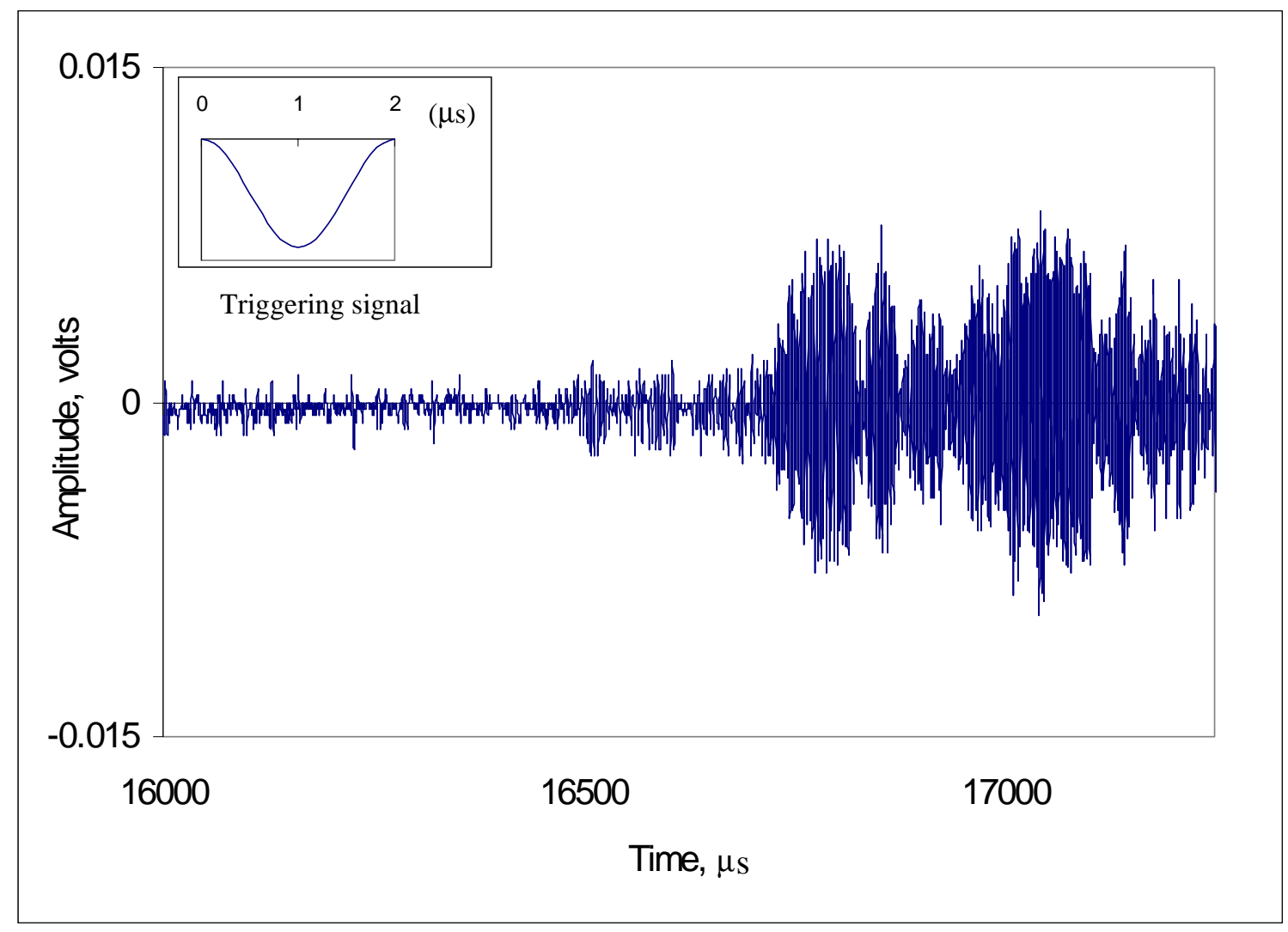

Figure 6.5 Arriving waveform recorded at the receiving end of the strand subjected to 20 kips tensile force. 


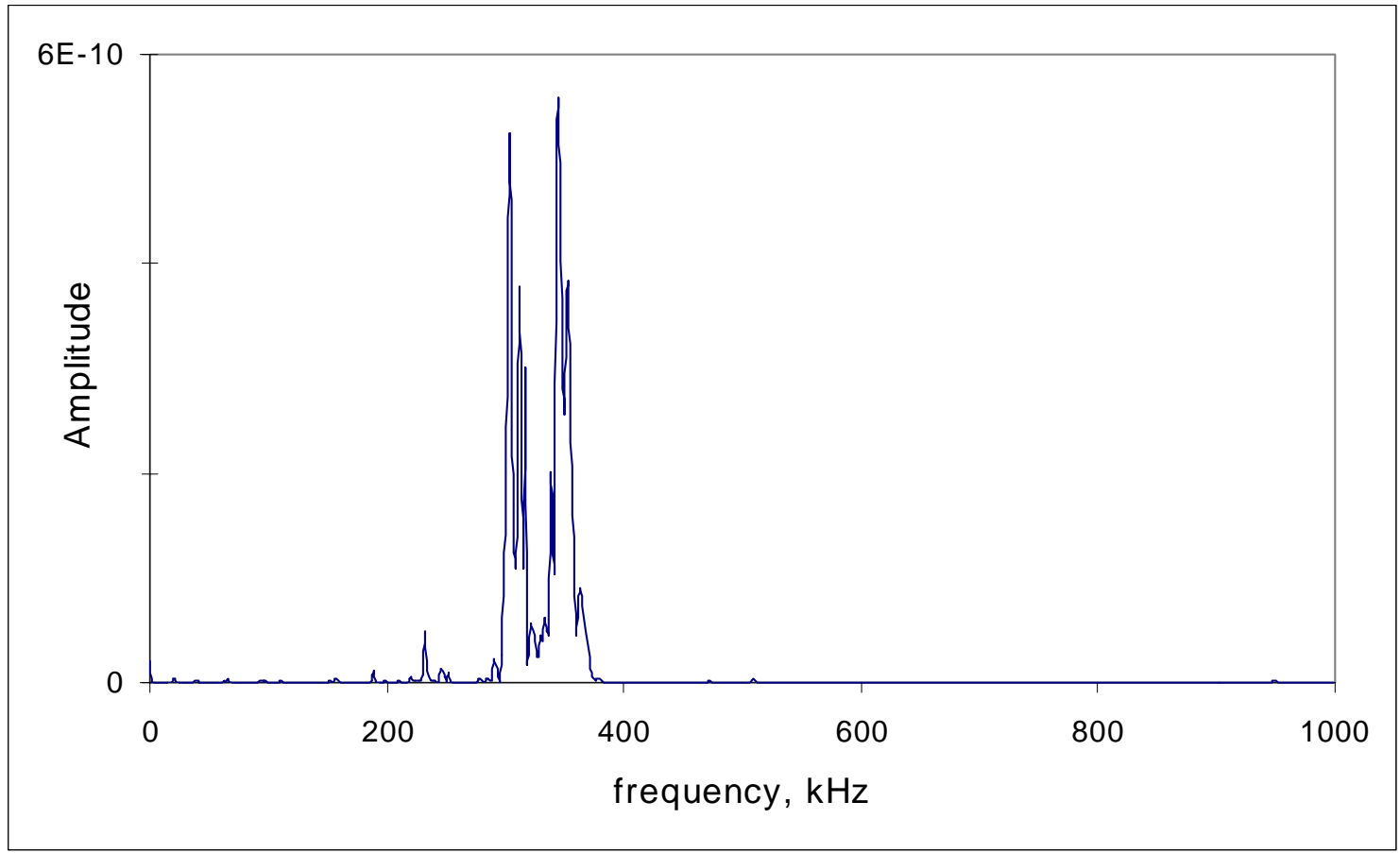

Figure 6.6 Frequency components of the recorded waveform due to 20 kips tensile force. 


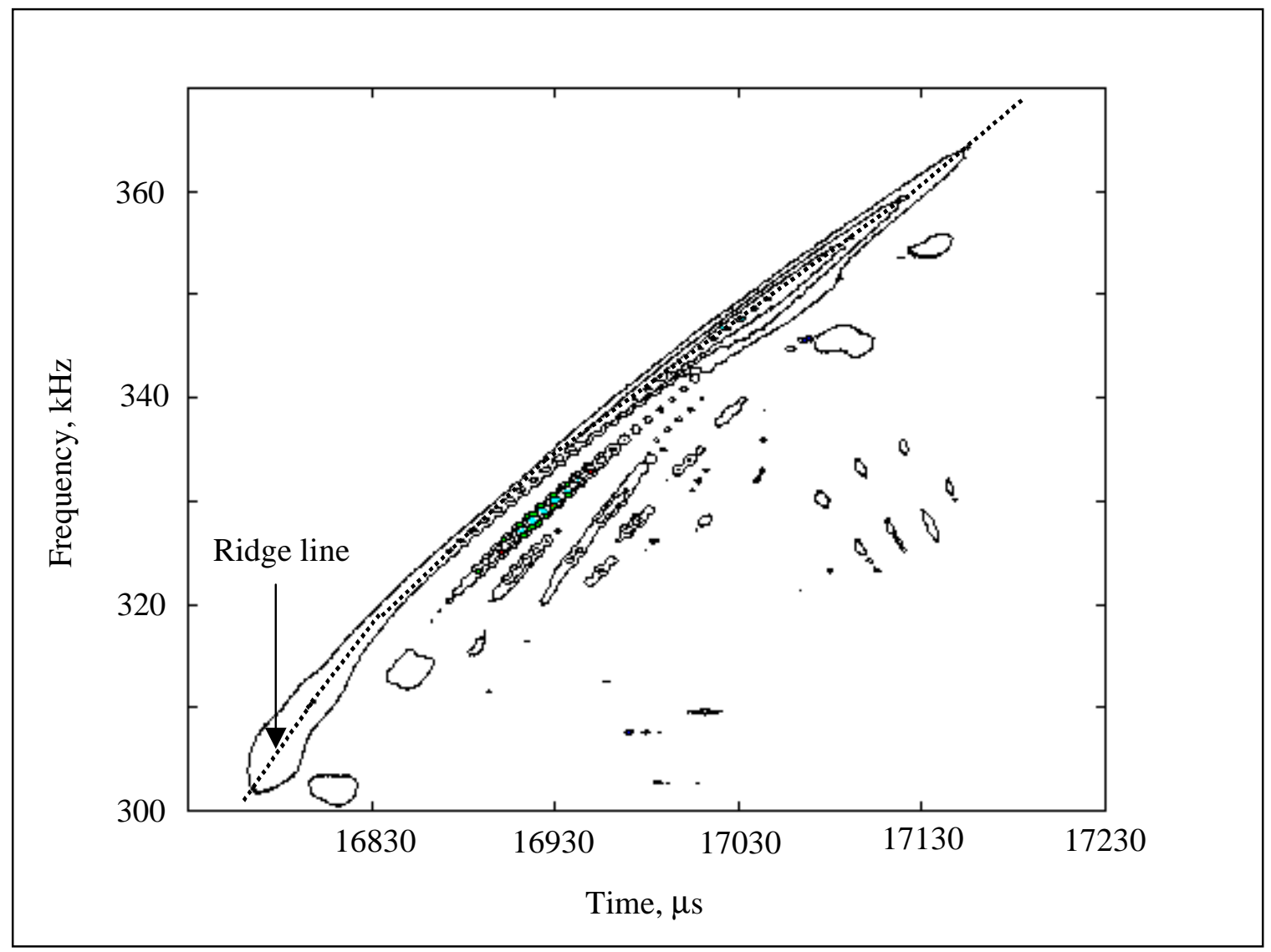

Figure 6.7 Time-frequency relationship of the recorded waveform due to 20 kips tensile force. 


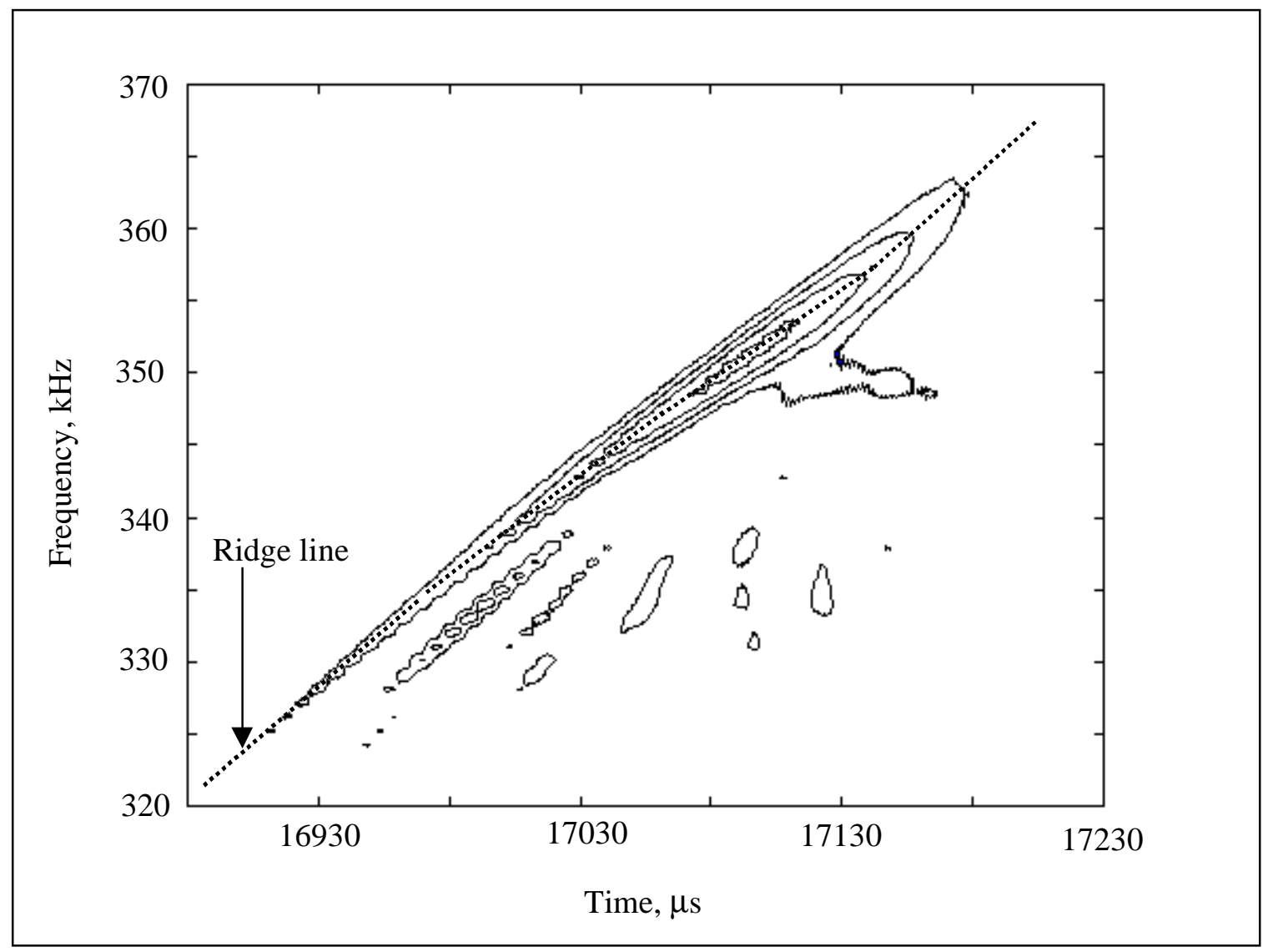

Figure 6.8 Time-frequency relationship of the recorded waveform due to 26 kips tensile force. 


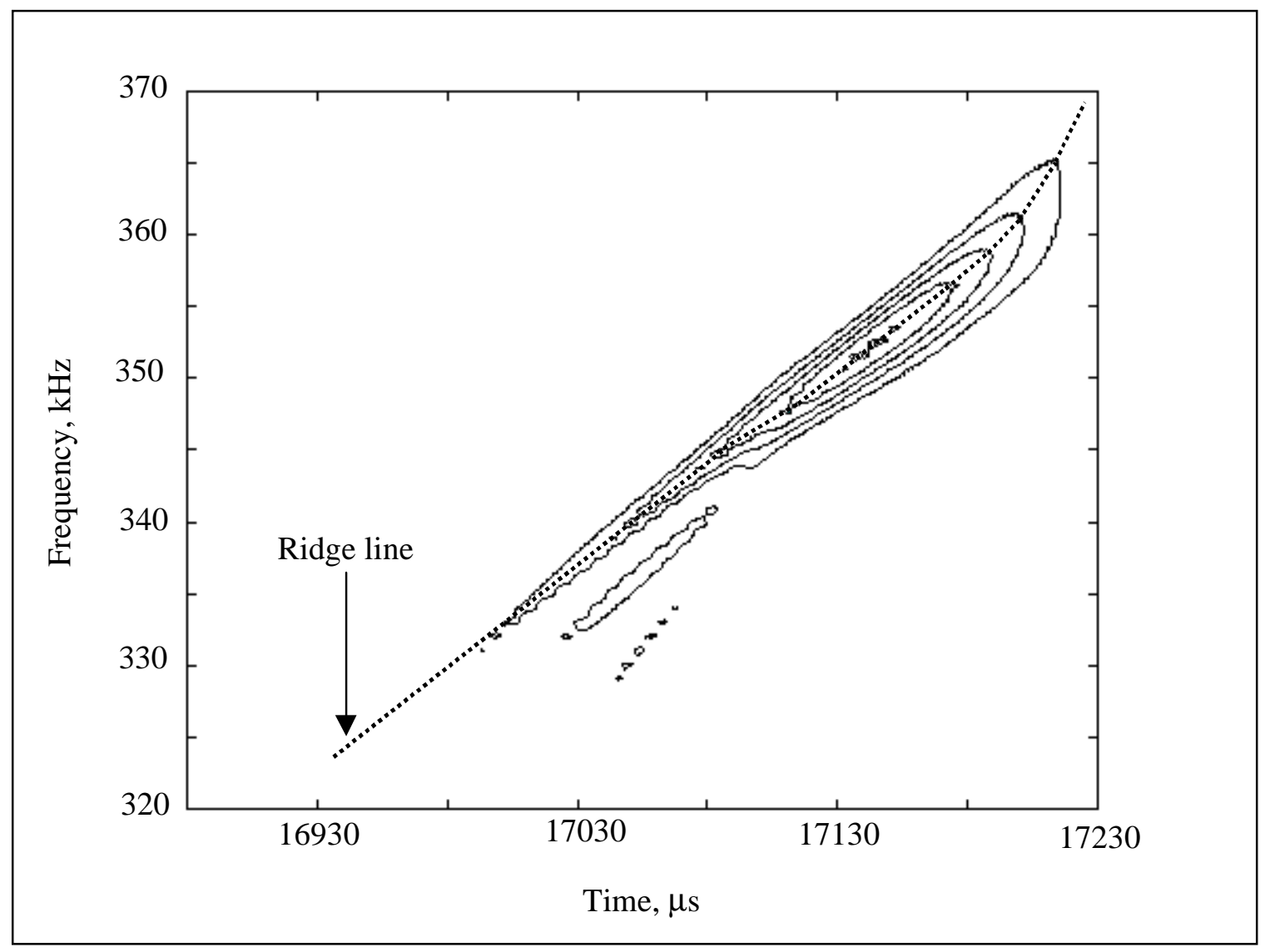

Figure 6.9 Time-frequency relationship of the recorded waveform due to $32 \mathrm{kips}$ tensile force. 


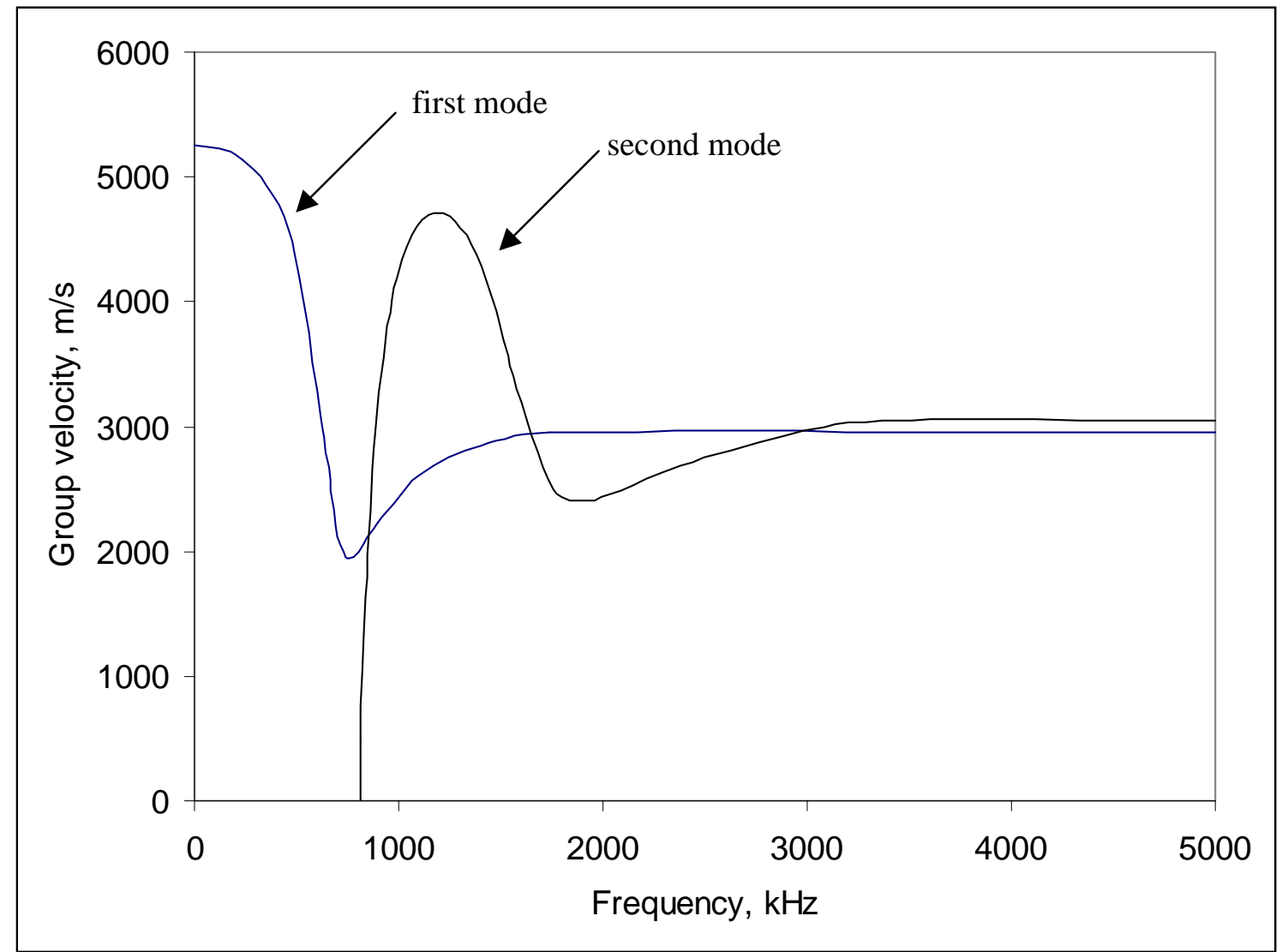

Figure 6.10 Group velocity of the first two longitudinal modes for the center wire $(\varnothing=$ $4.4 \mathrm{~mm})$. There is no tensile force applied. 


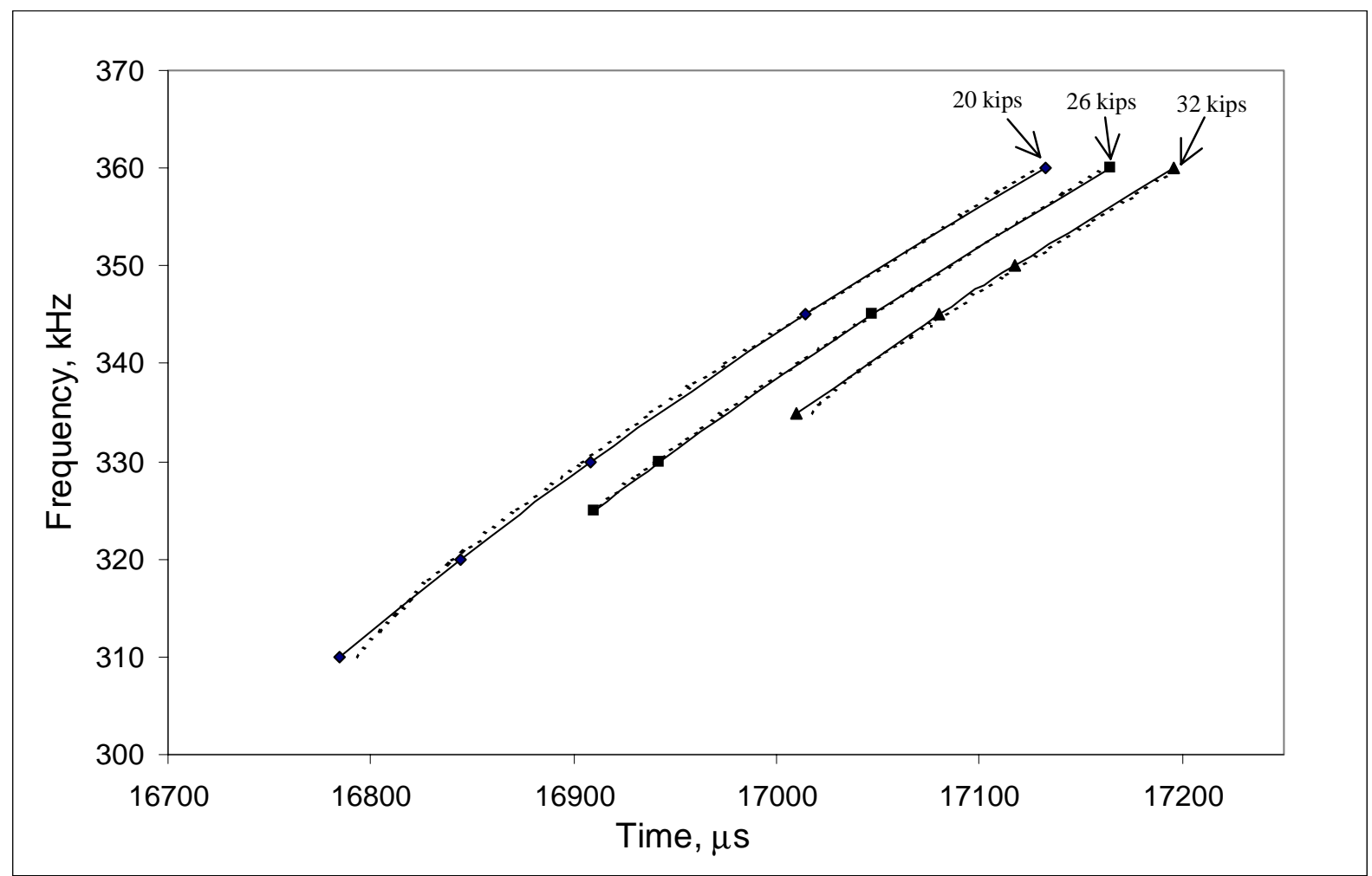

Figure 6.11 Time-frequency relationships of waveforms due to $20 \mathrm{kips}, 26 \mathrm{kips}$, and 32 kips tensile forces. Dotted lines represent experimental results. Solid lines represent numerical results. 


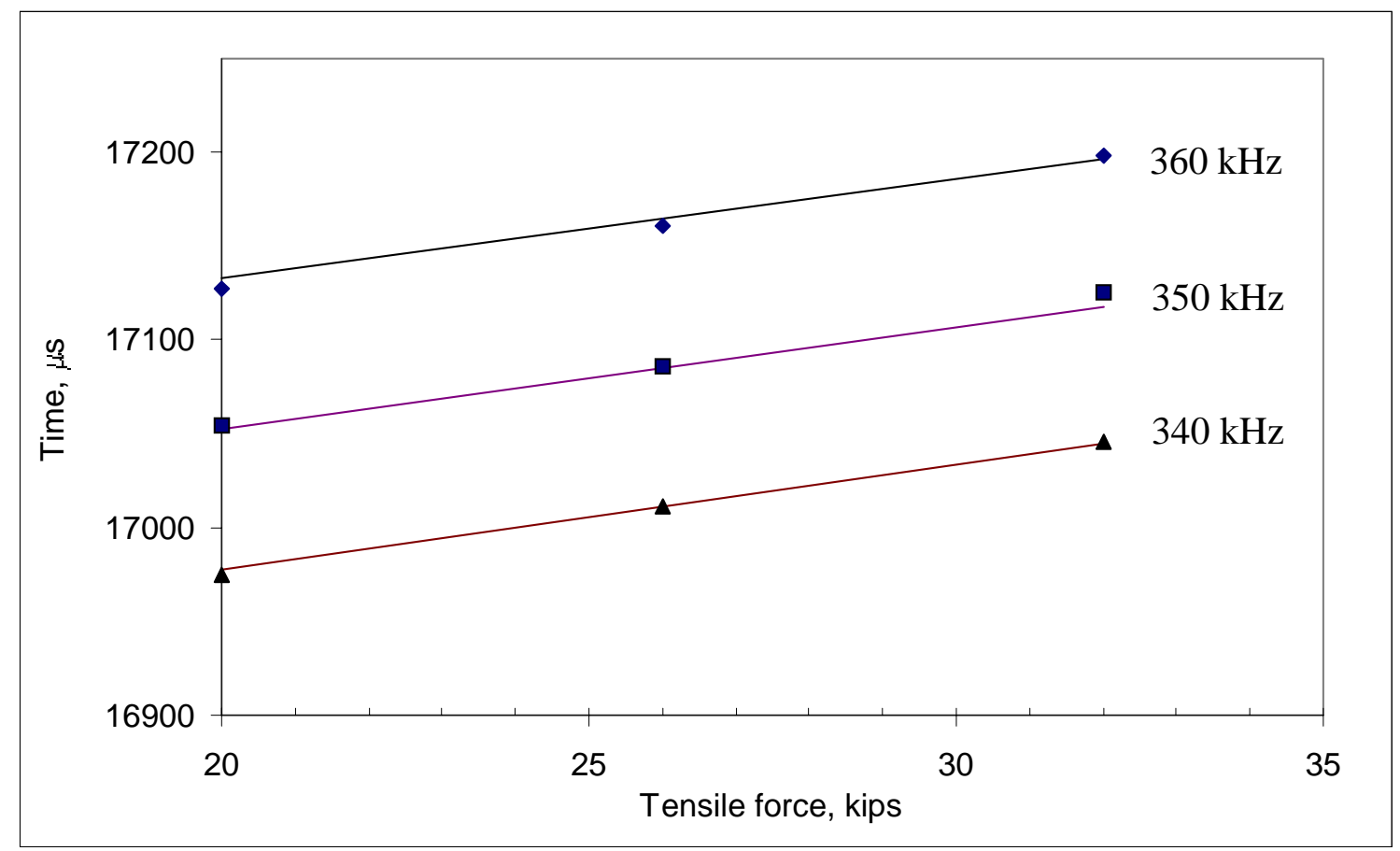

Figure 6.12 Arrival time of $340 \mathrm{kHz}, 350 \mathrm{kHz}$, and $360 \mathrm{kHz}$ frequency components due to increasing tensile forces. Symbols represent experimental data. Solid lines represent analytical results. 


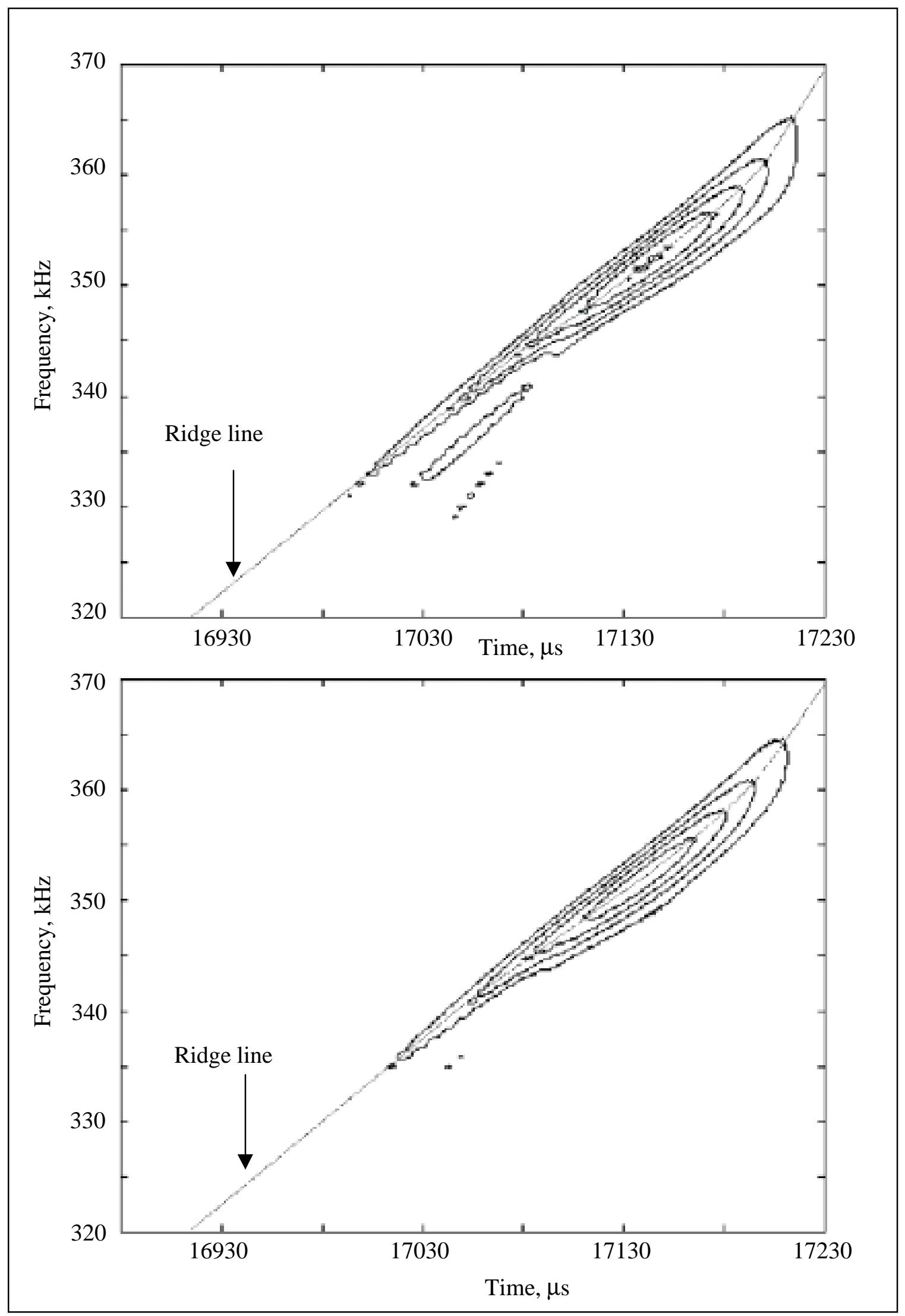

Figure 6.13 Reproducibility of two recorded waveforms due to 32 kips tensile force. 


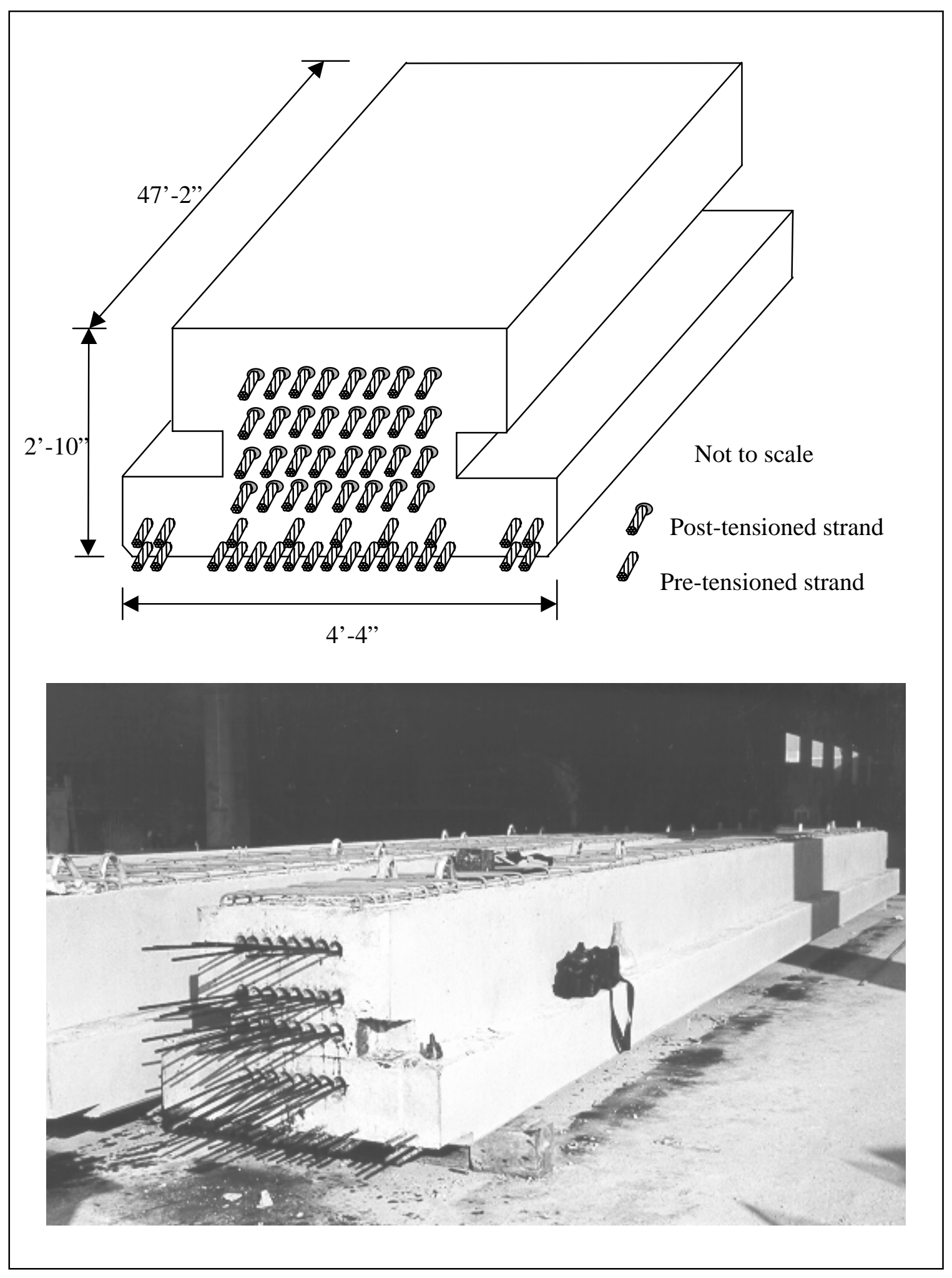

Figure 6.14 Inverted Tee Girder 


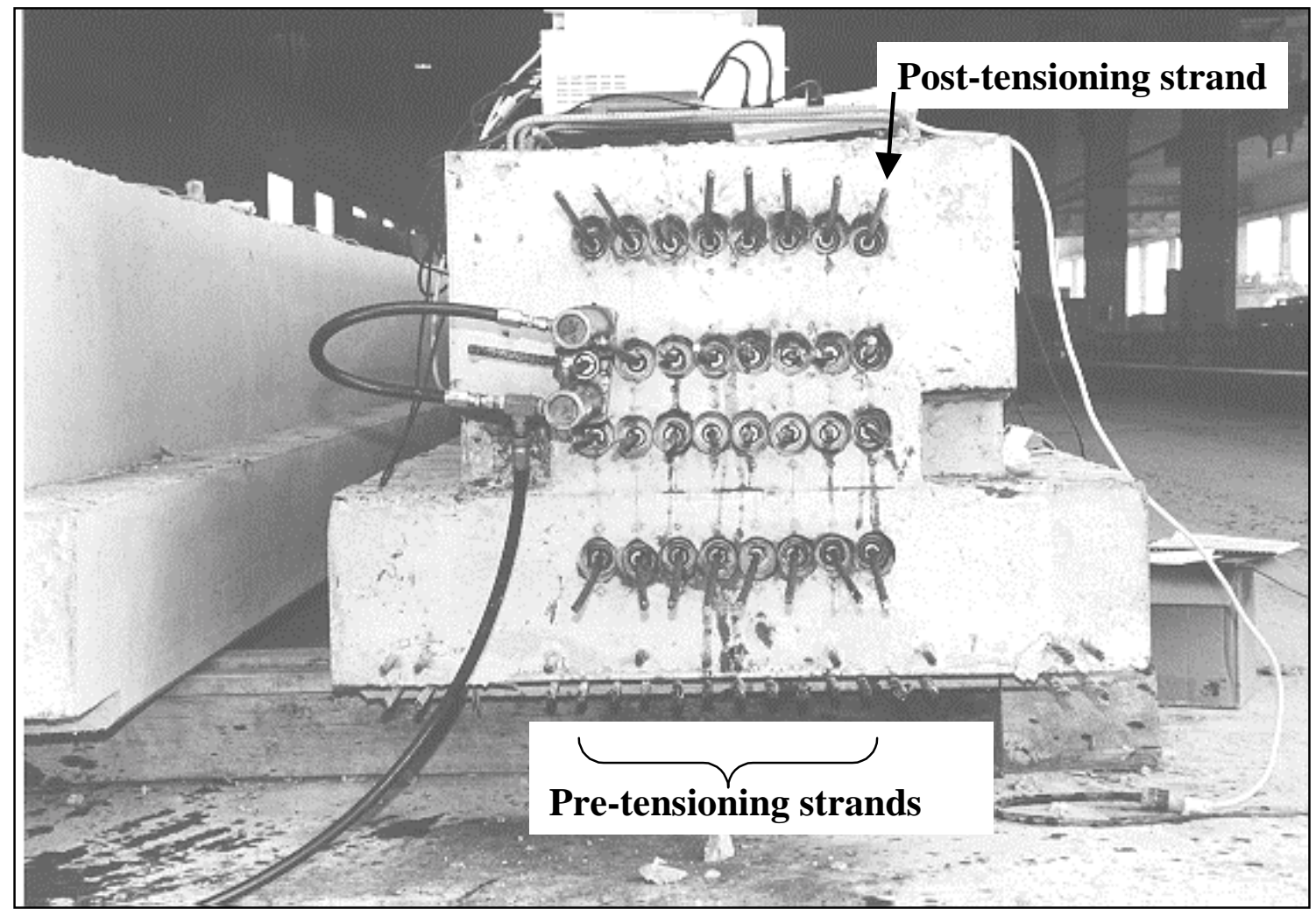

Figure 6.15 Loading end of the prestressing strands 


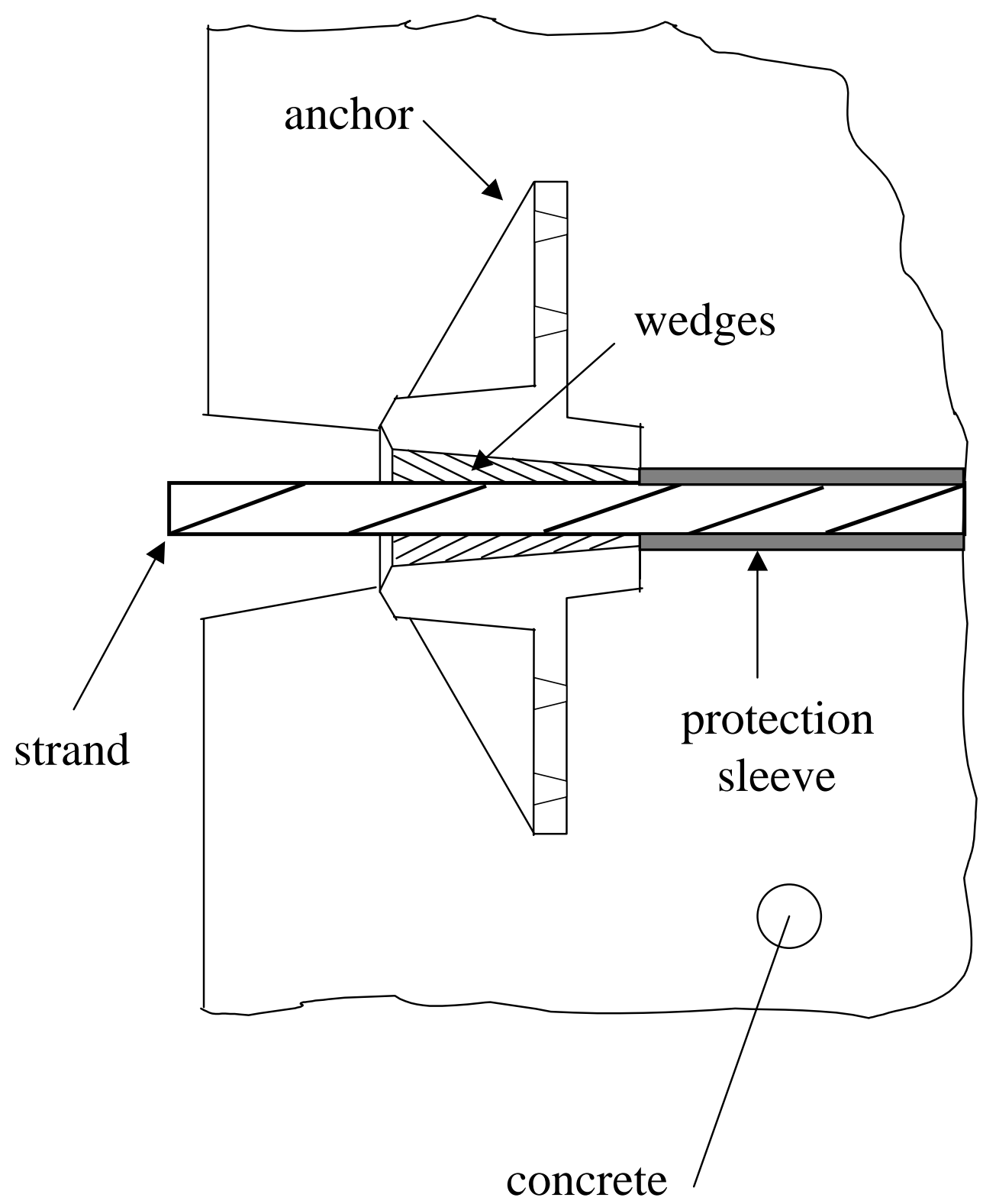

Figure 6.16 Monostrand system 


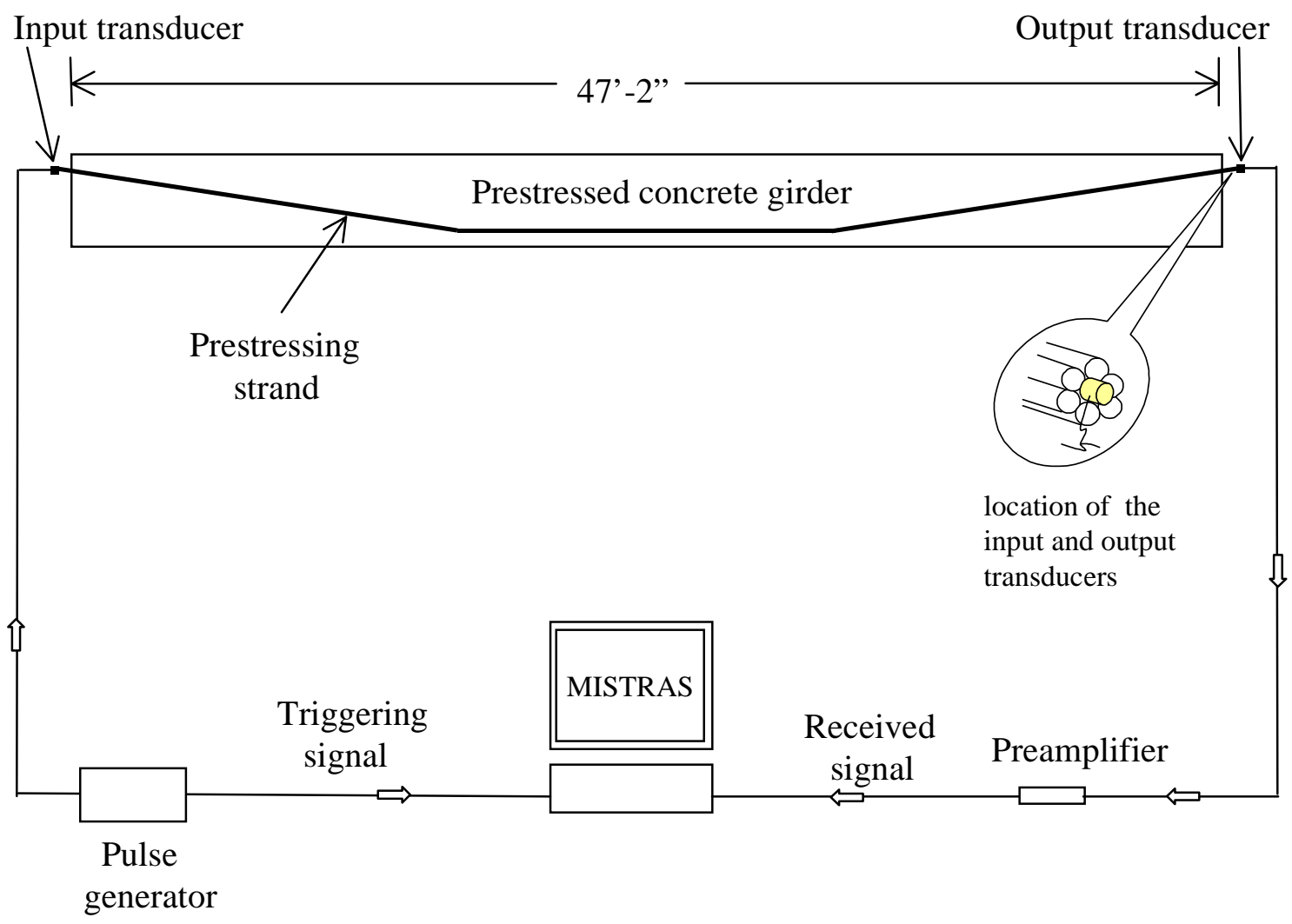

Figure 6.17 Experimental setup using pulse generator 


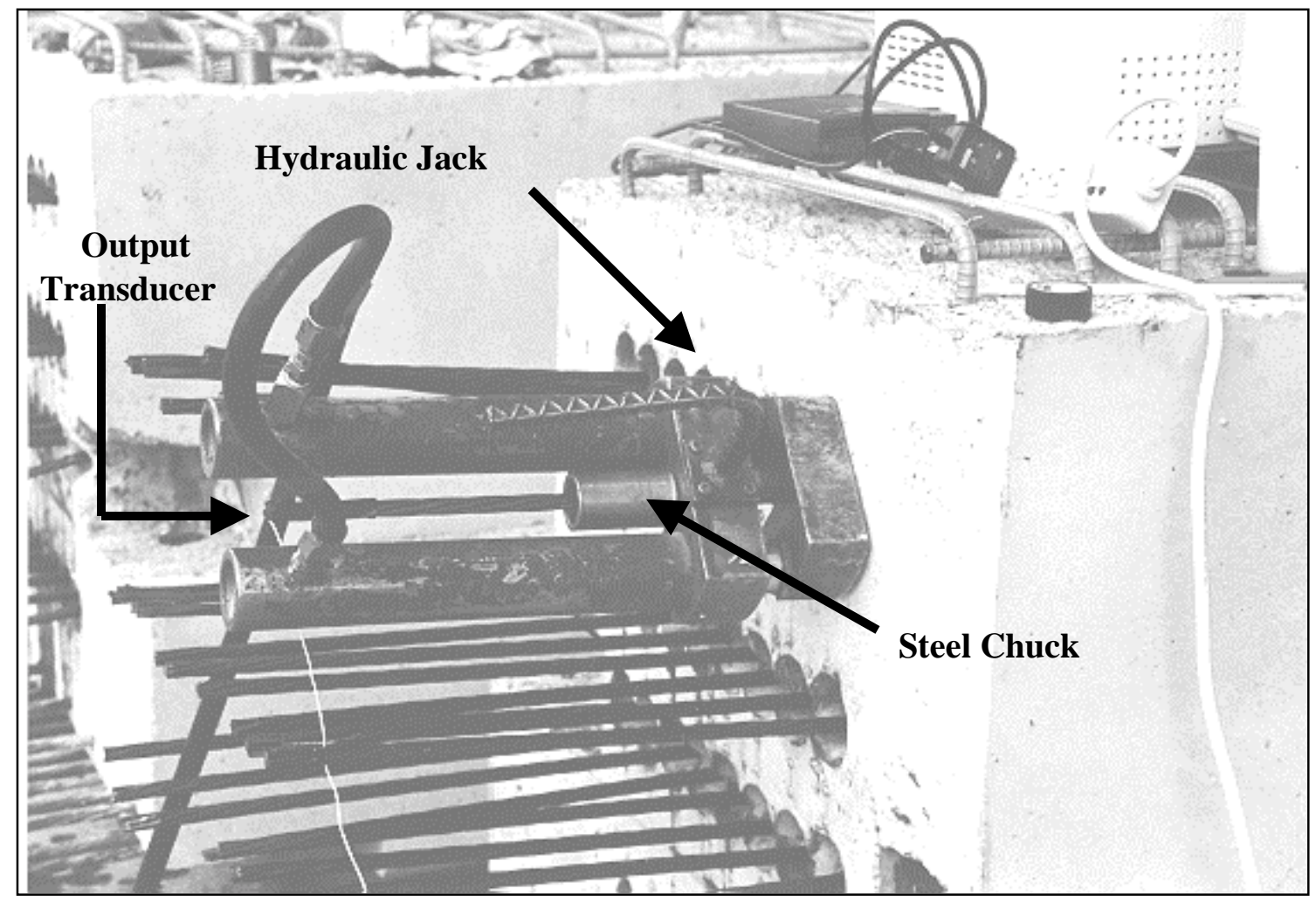

Figure 6.18 Experimental setup at the receiving end of the strand 


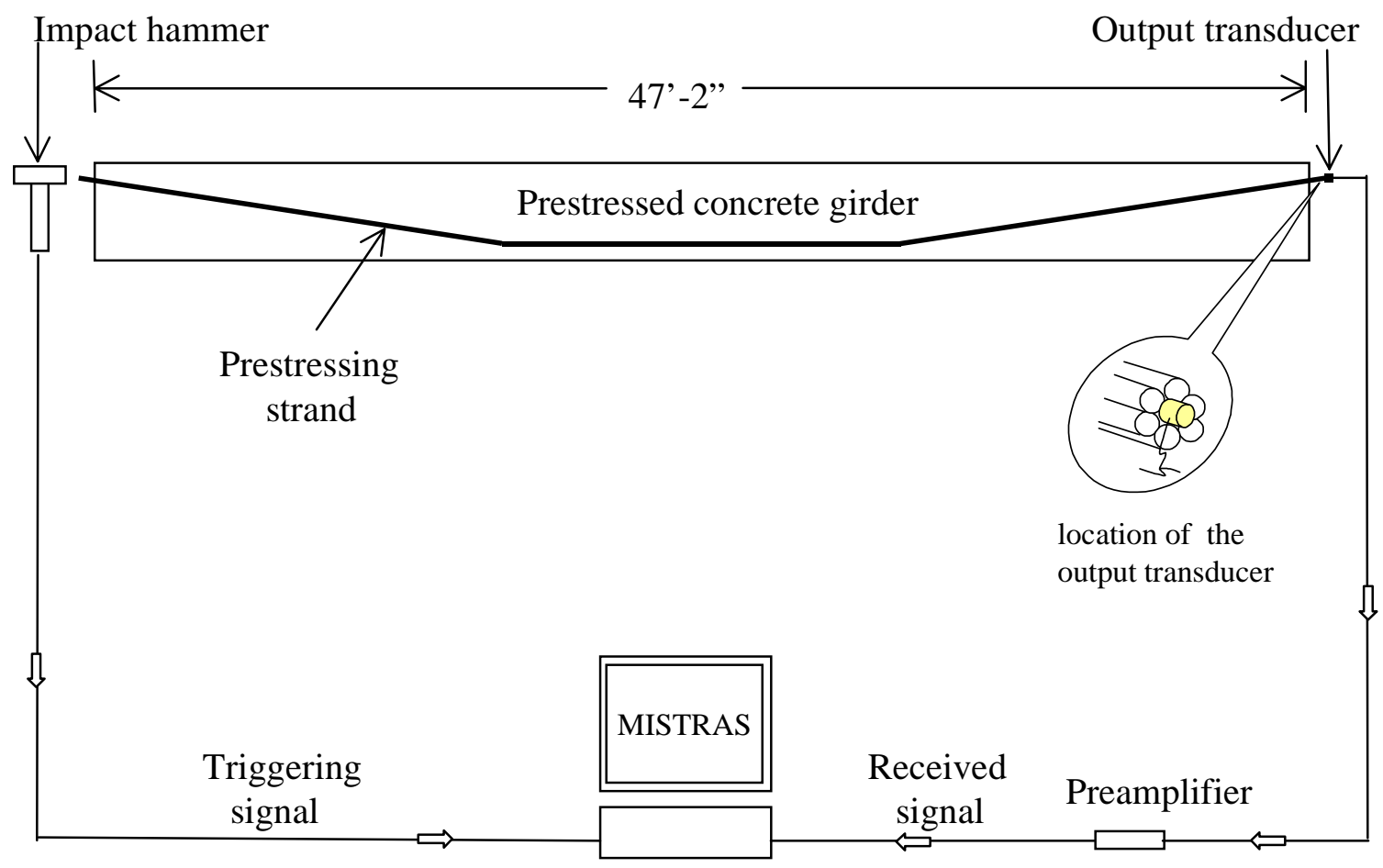

Figure 6.19 Experimental setup using impact hammer 


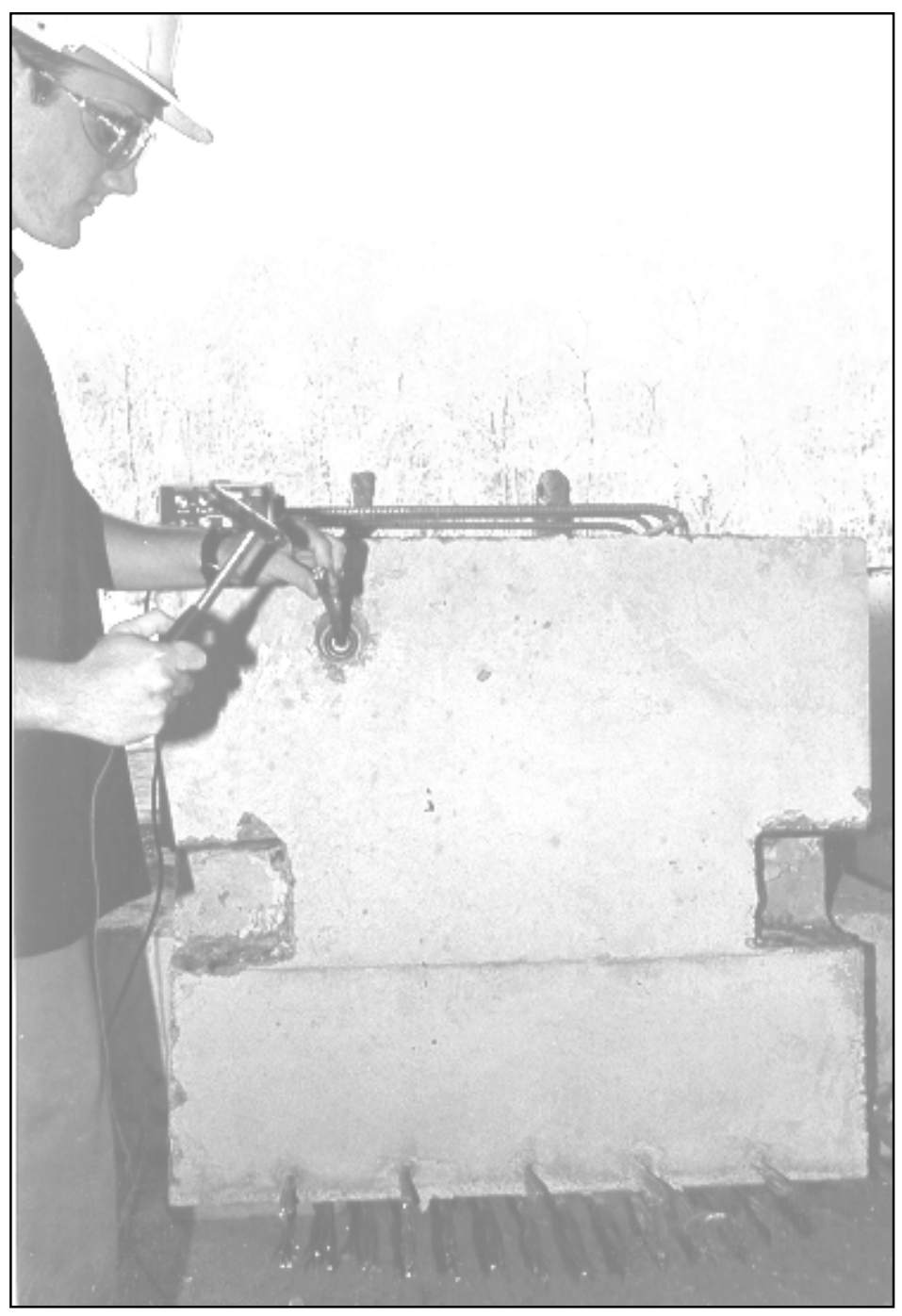

Figure 6.20 Impact excitation 


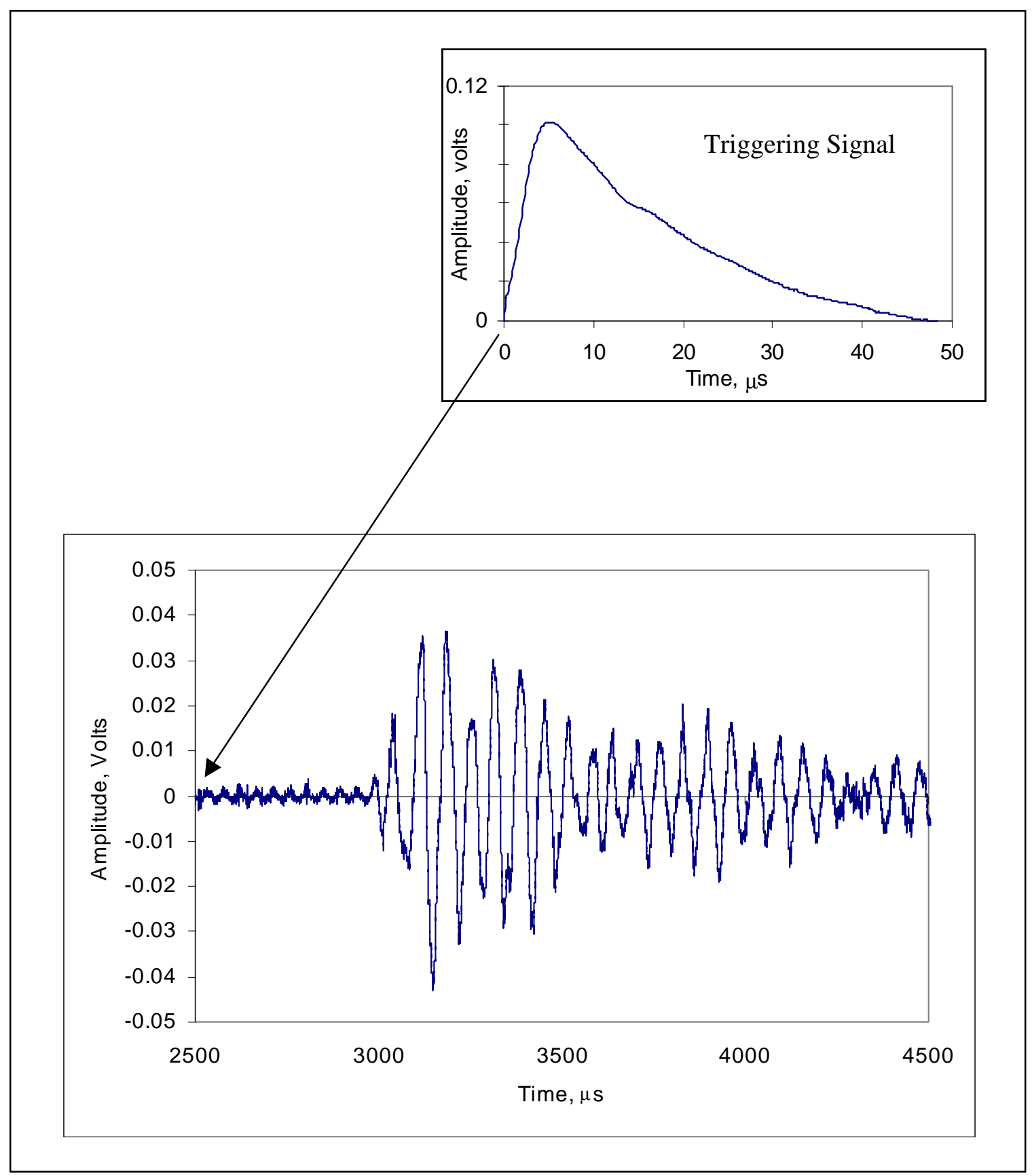

Figure 6.21 Arriving stress wave signal due to 10 kips tensile force 


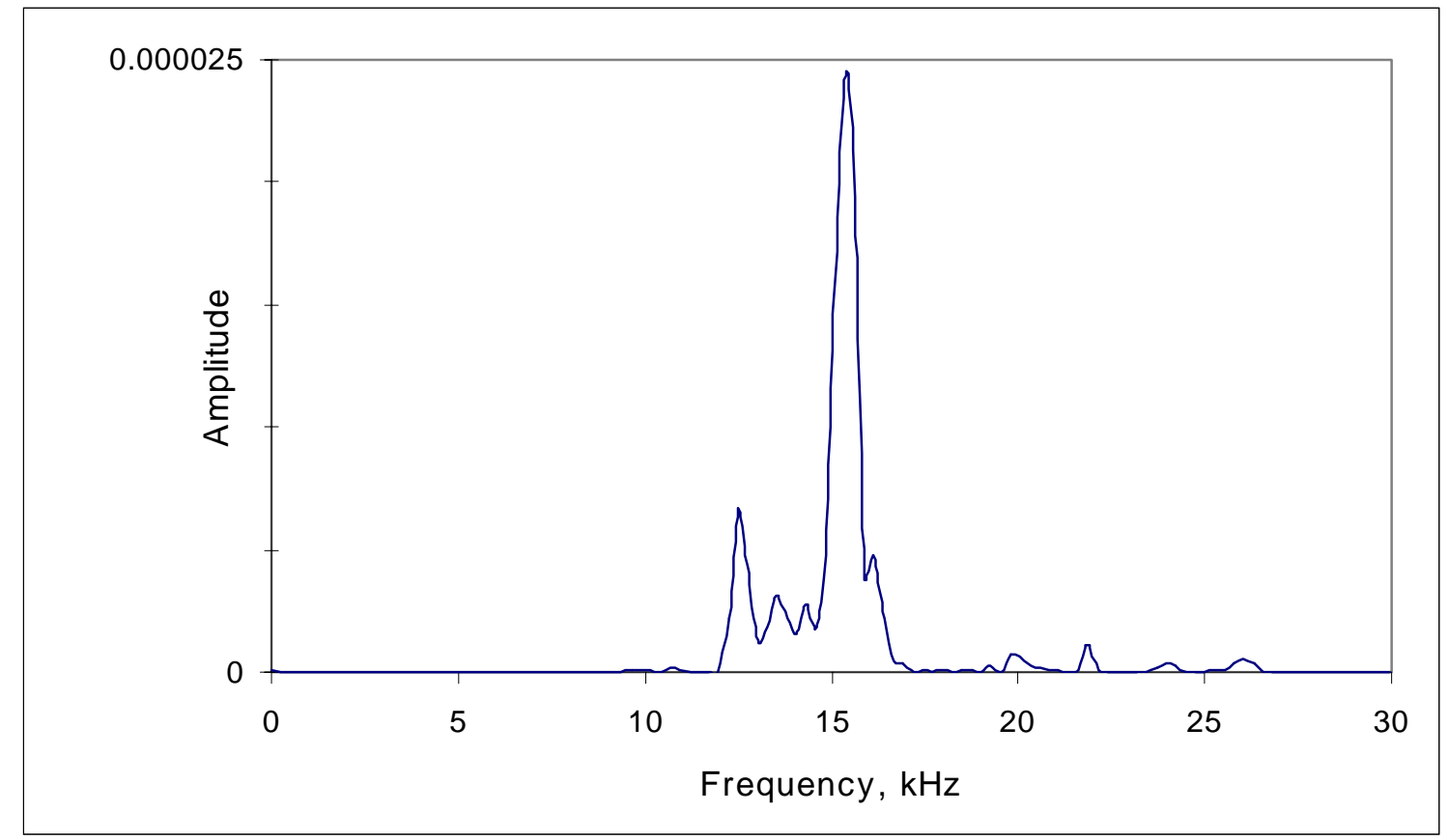

Figure 6.22 Typical frequency components of detected waveforms due to 10 kips tensile force 


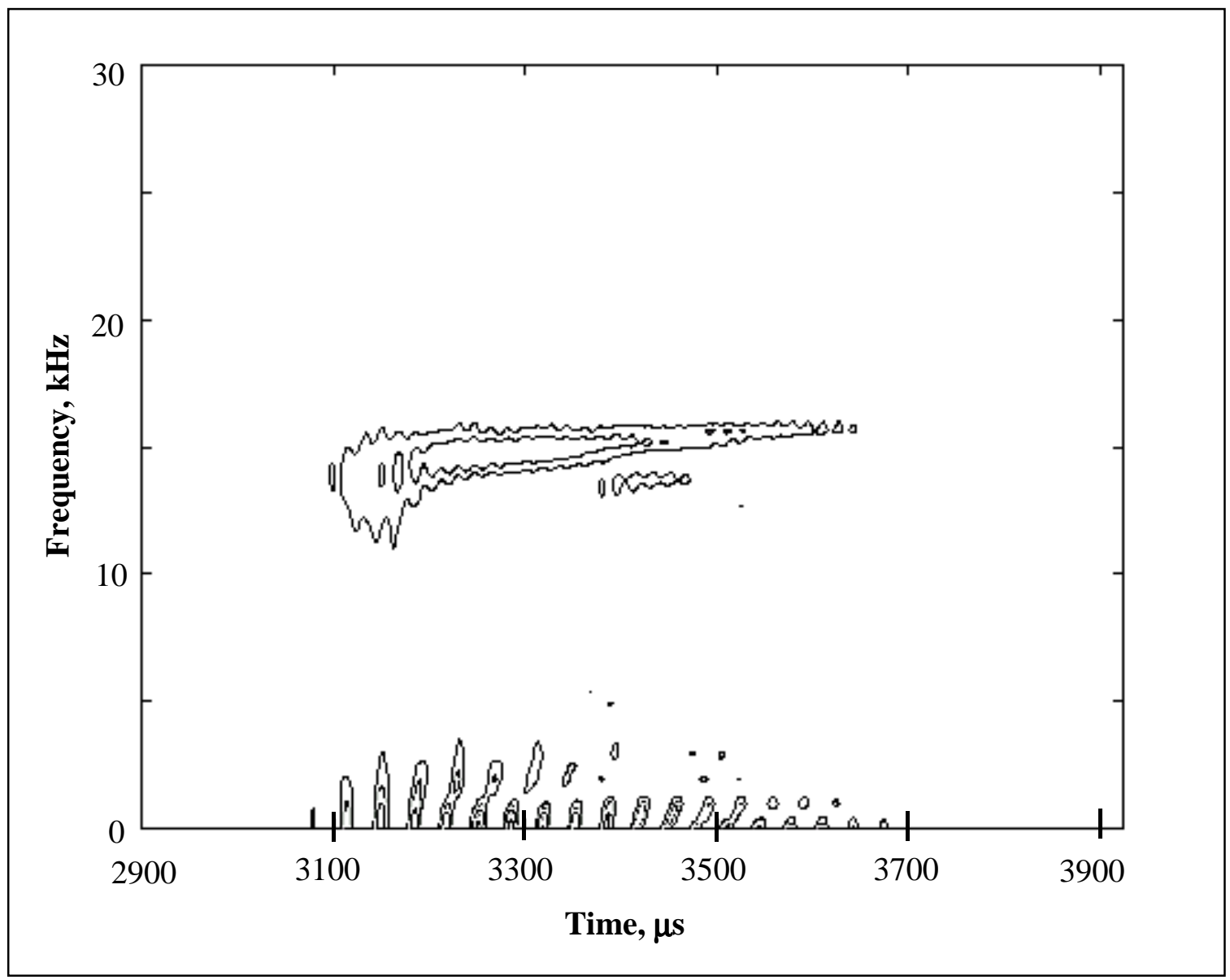

Figure 6.23 WVT plot of the detected waveform due to 10 kips tensile force 


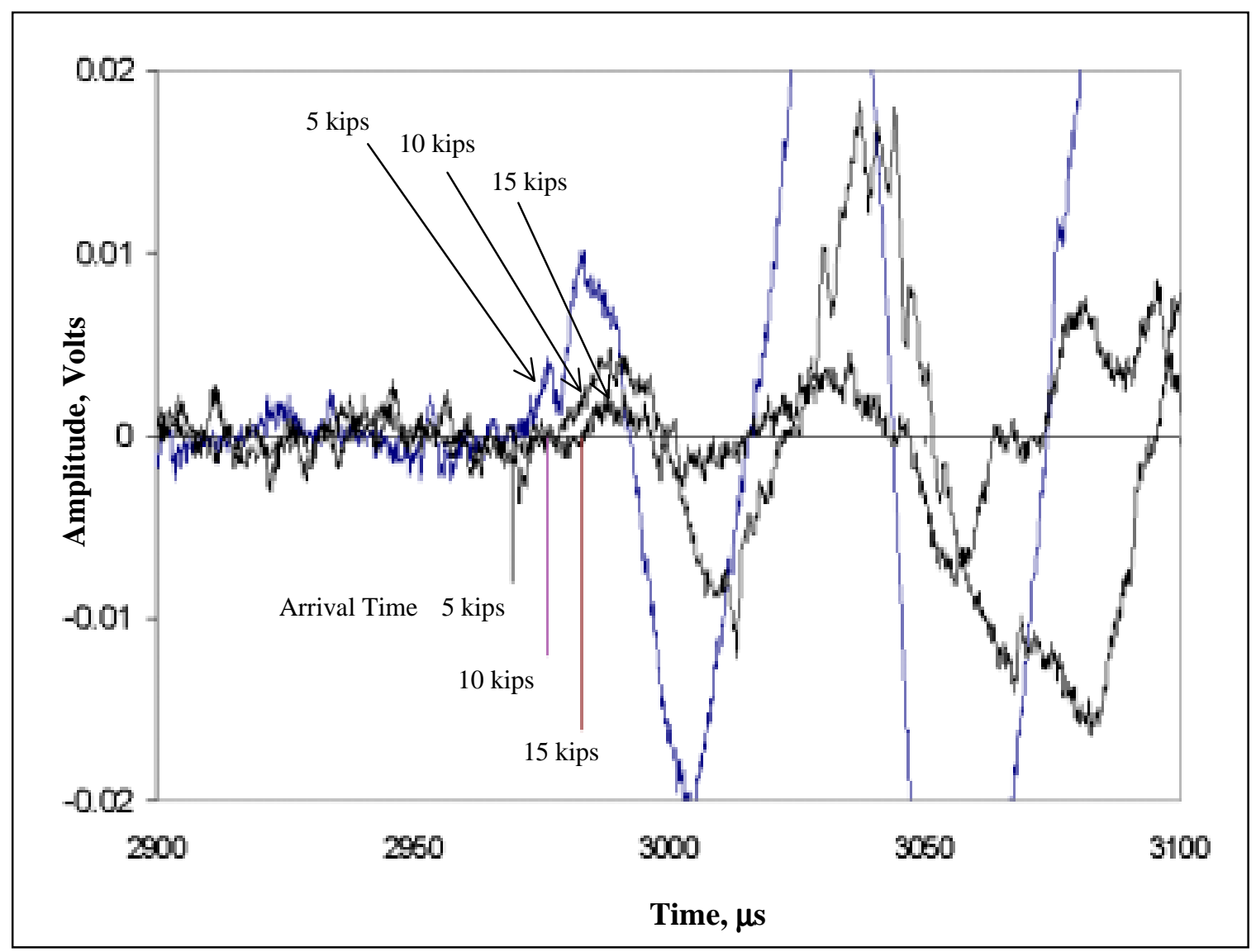

Figure 6.24 Time shift of the waveform due to changes in tensile force 


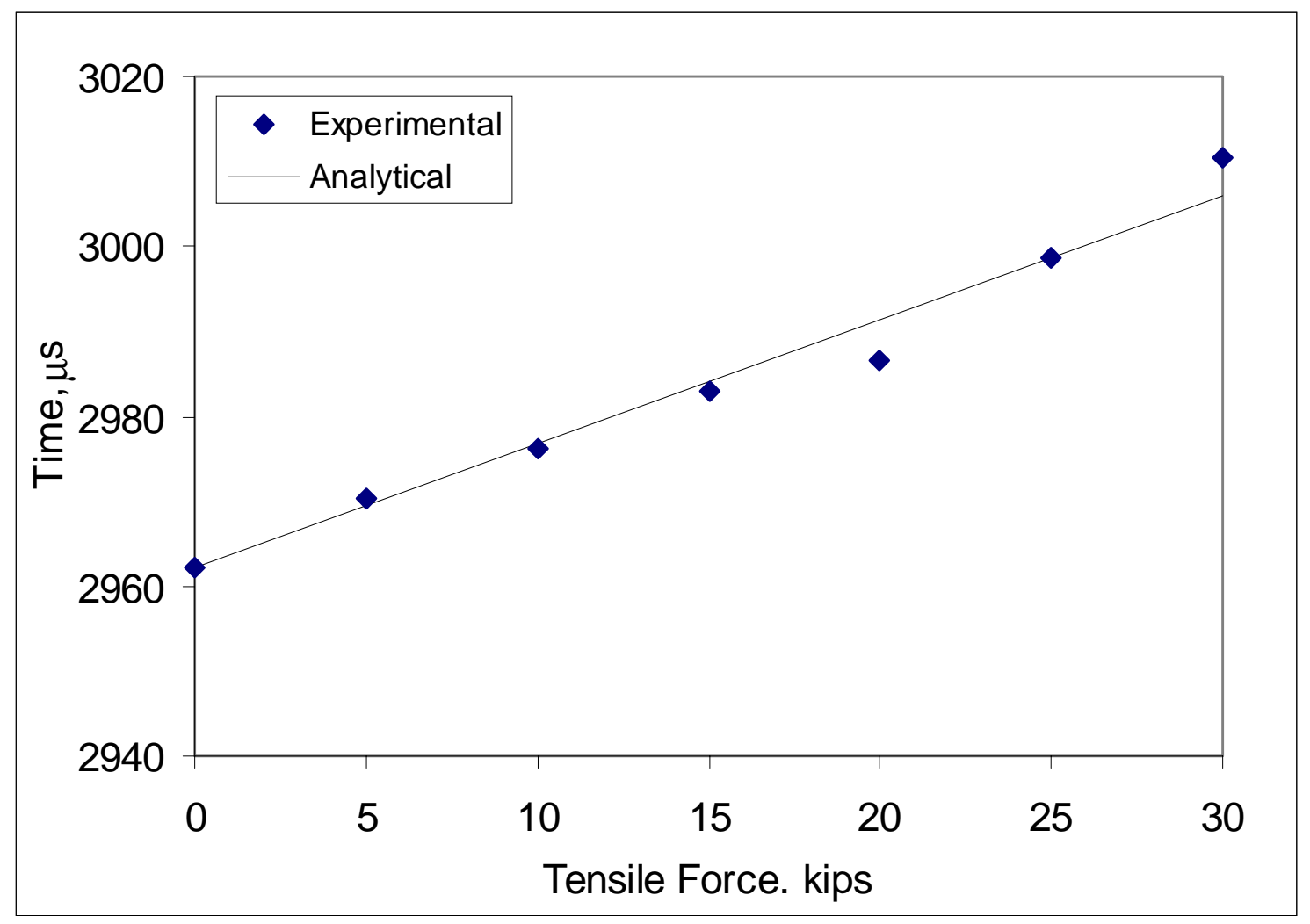

Figure 6.25 Arrival times of the waveforms due to different tensile forces 


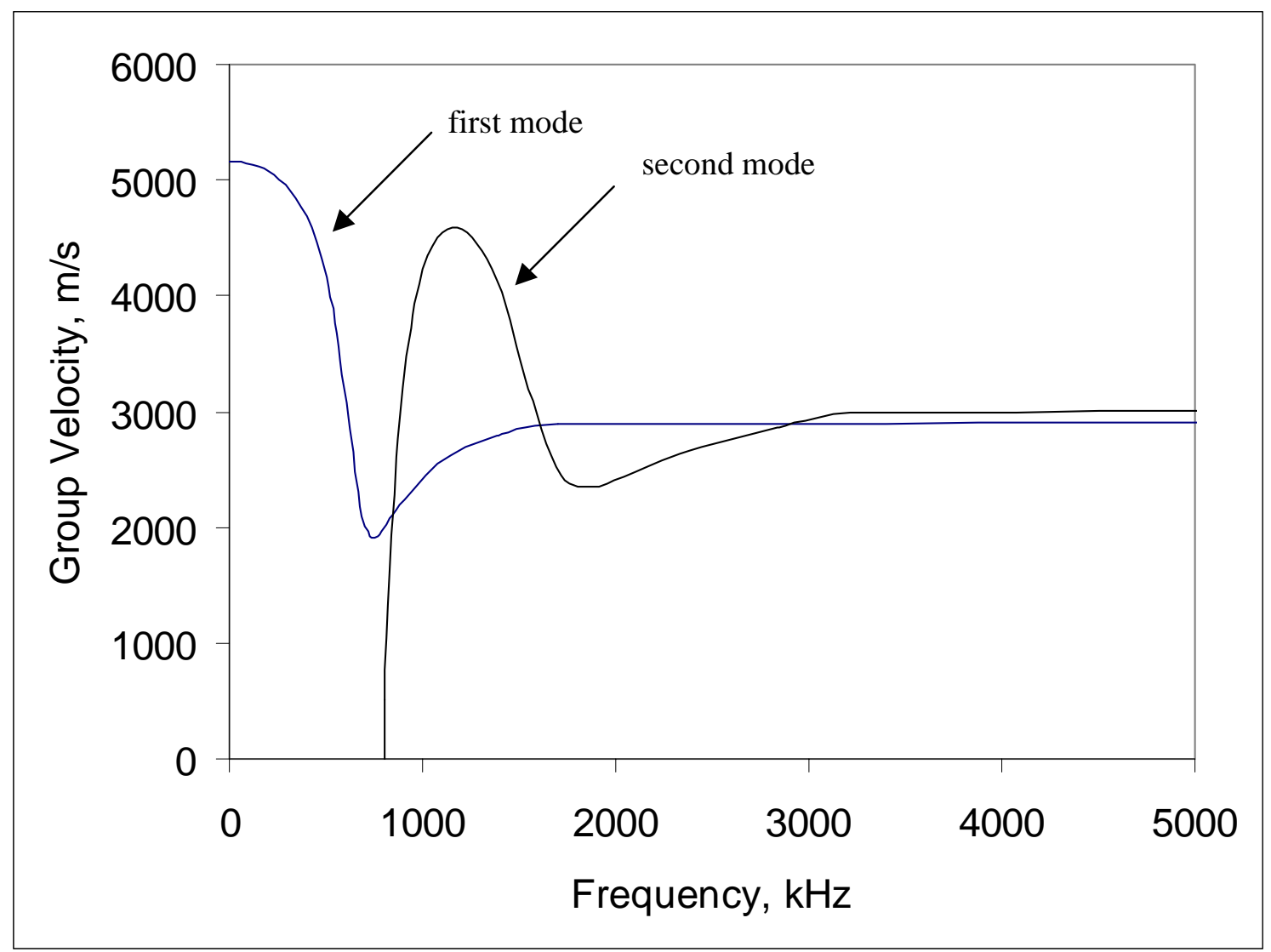

Figure 6.26 Group velocity of the first two longitudinal modes for the center wire $(\varnothing=$ $4.4 \mathrm{~mm})$. There is no tensile force applied. 


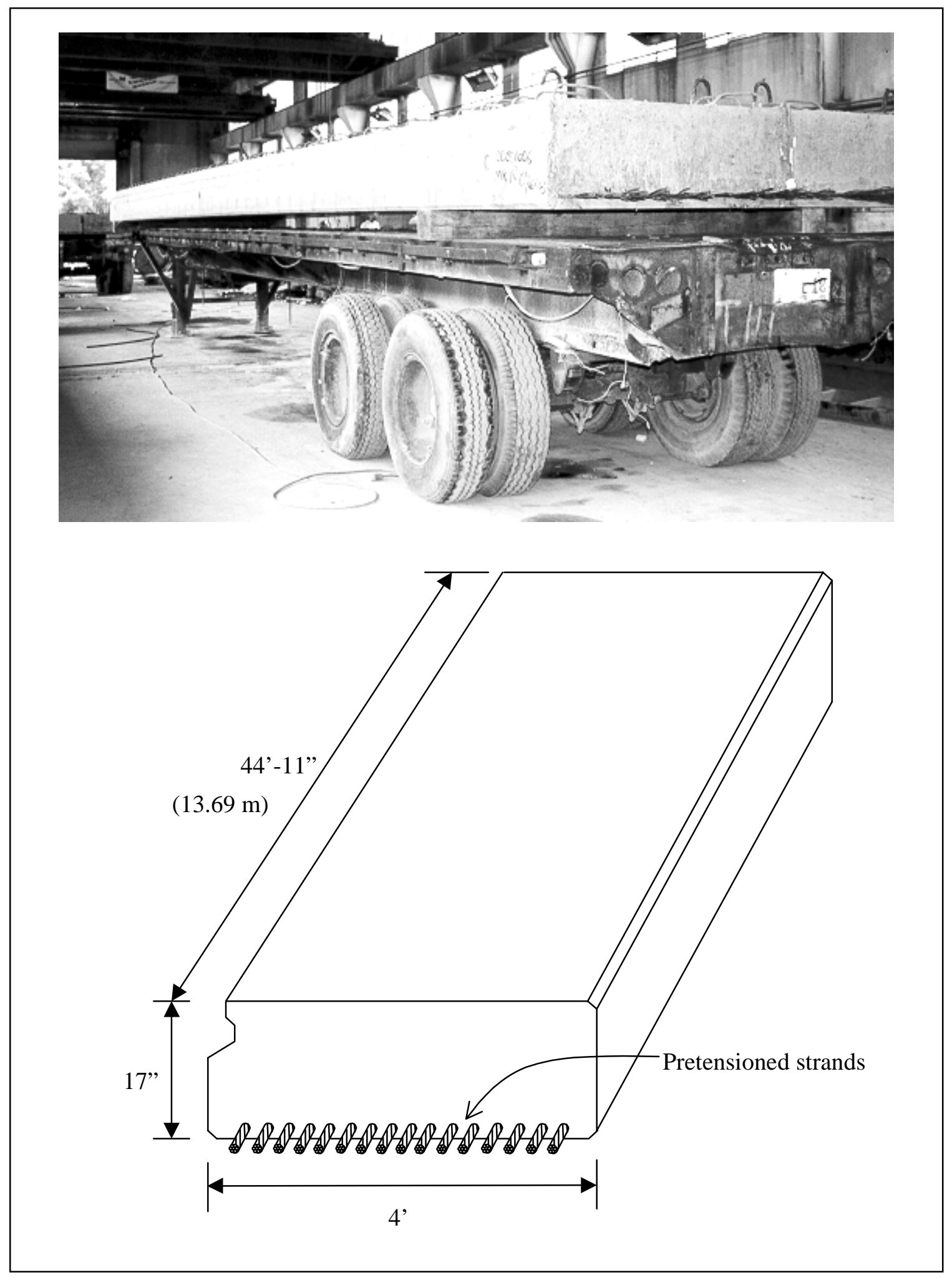

Figure 6.27 Pretensioned concrete box girder 


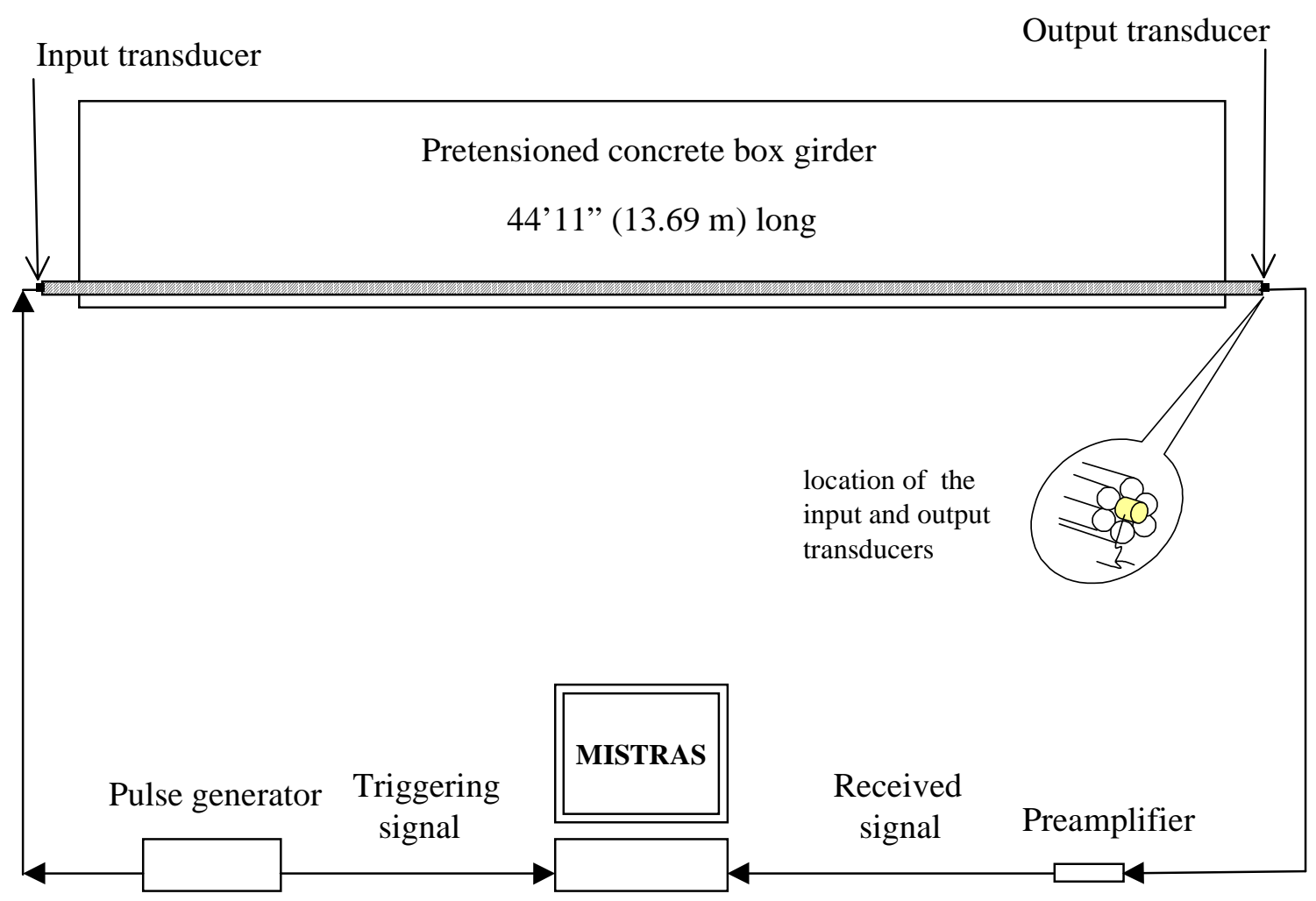

Figure 6.28 Experimental setup for pretensioned concrete box girder 


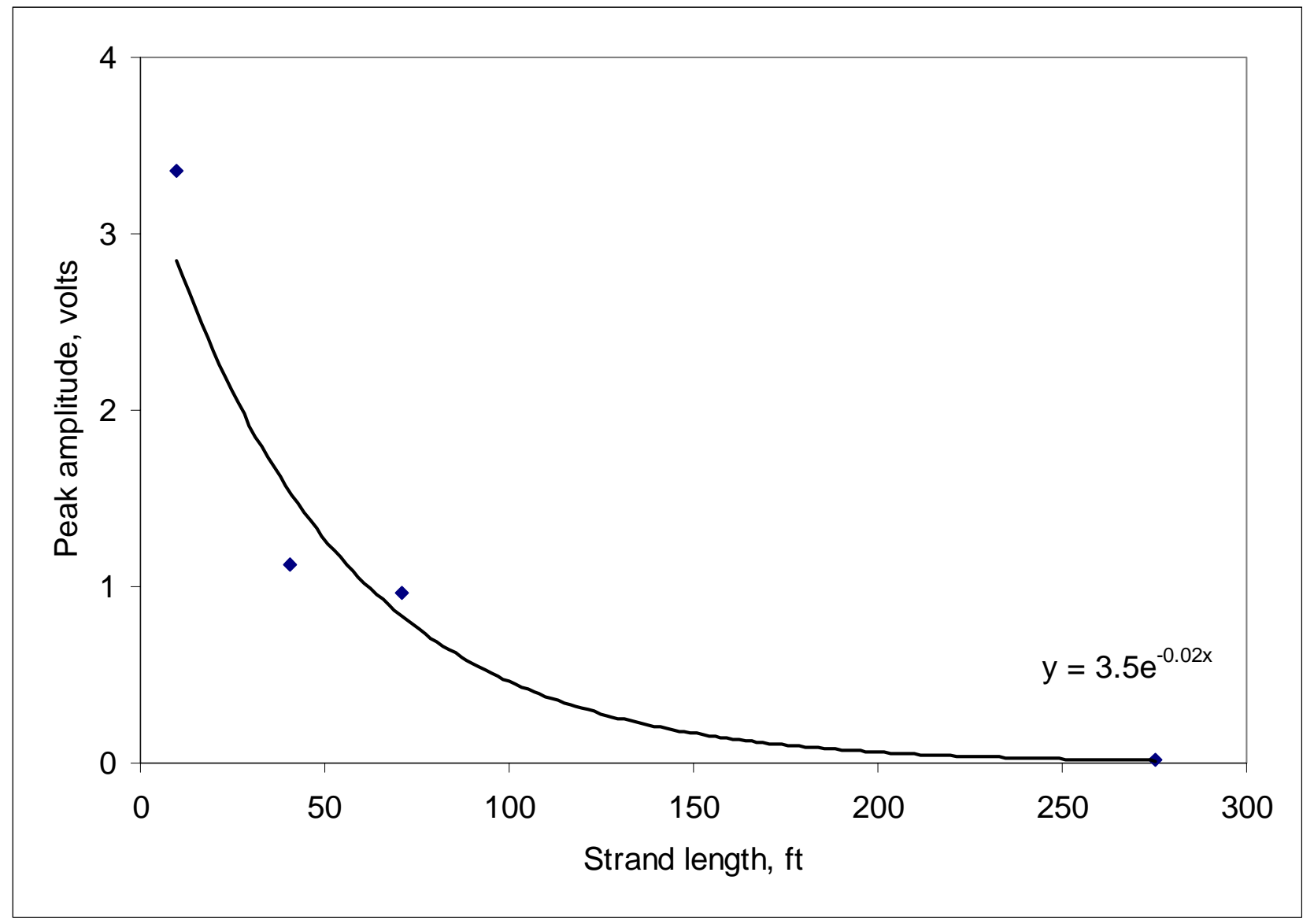

Figure 6.29 Peak amplitudes of recorded stress waves due to different strand length 


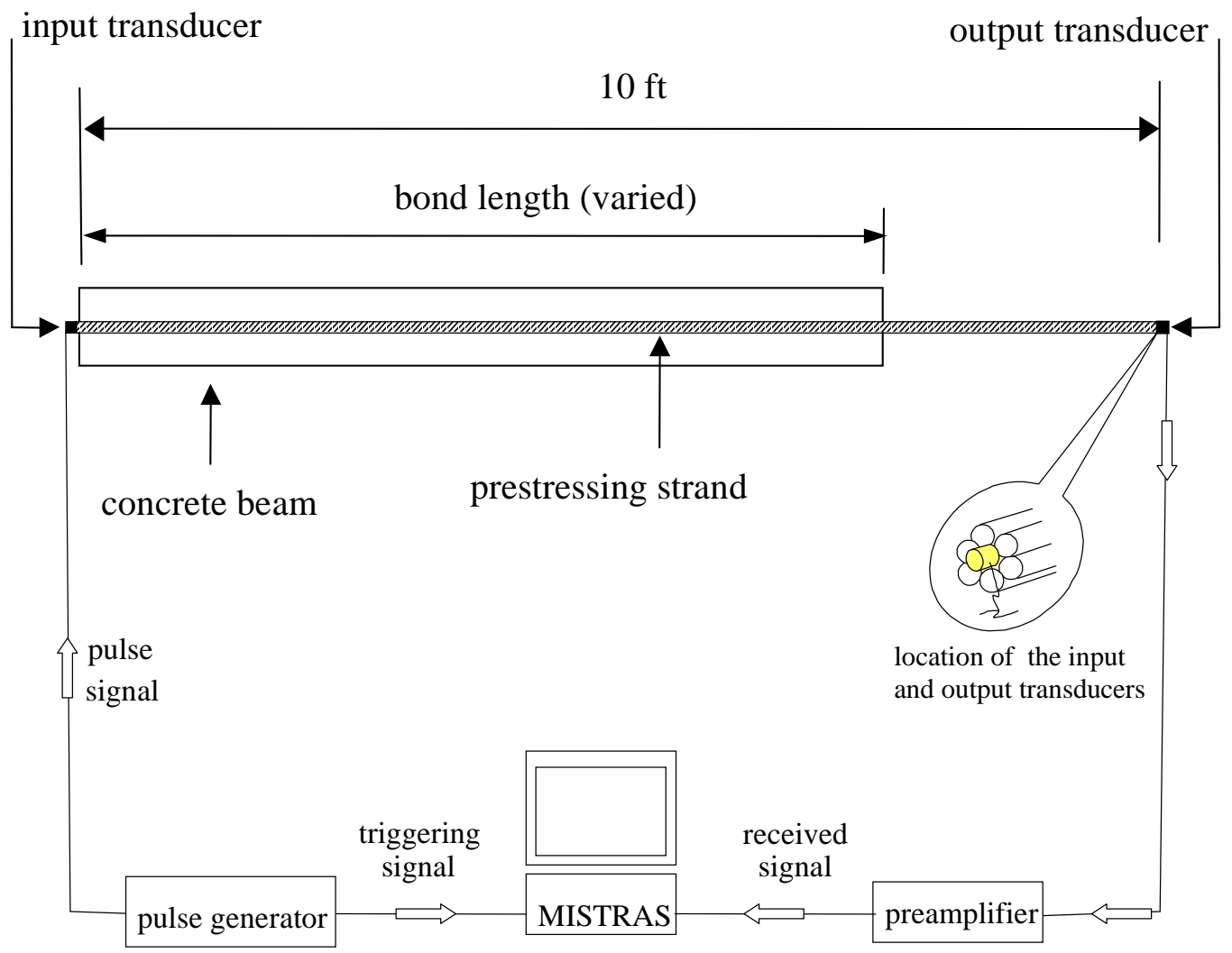

Figure 6.30 Experimental setup for determining the attenuation of the stress wave due to bonding between the seven-wire strand and concrete 

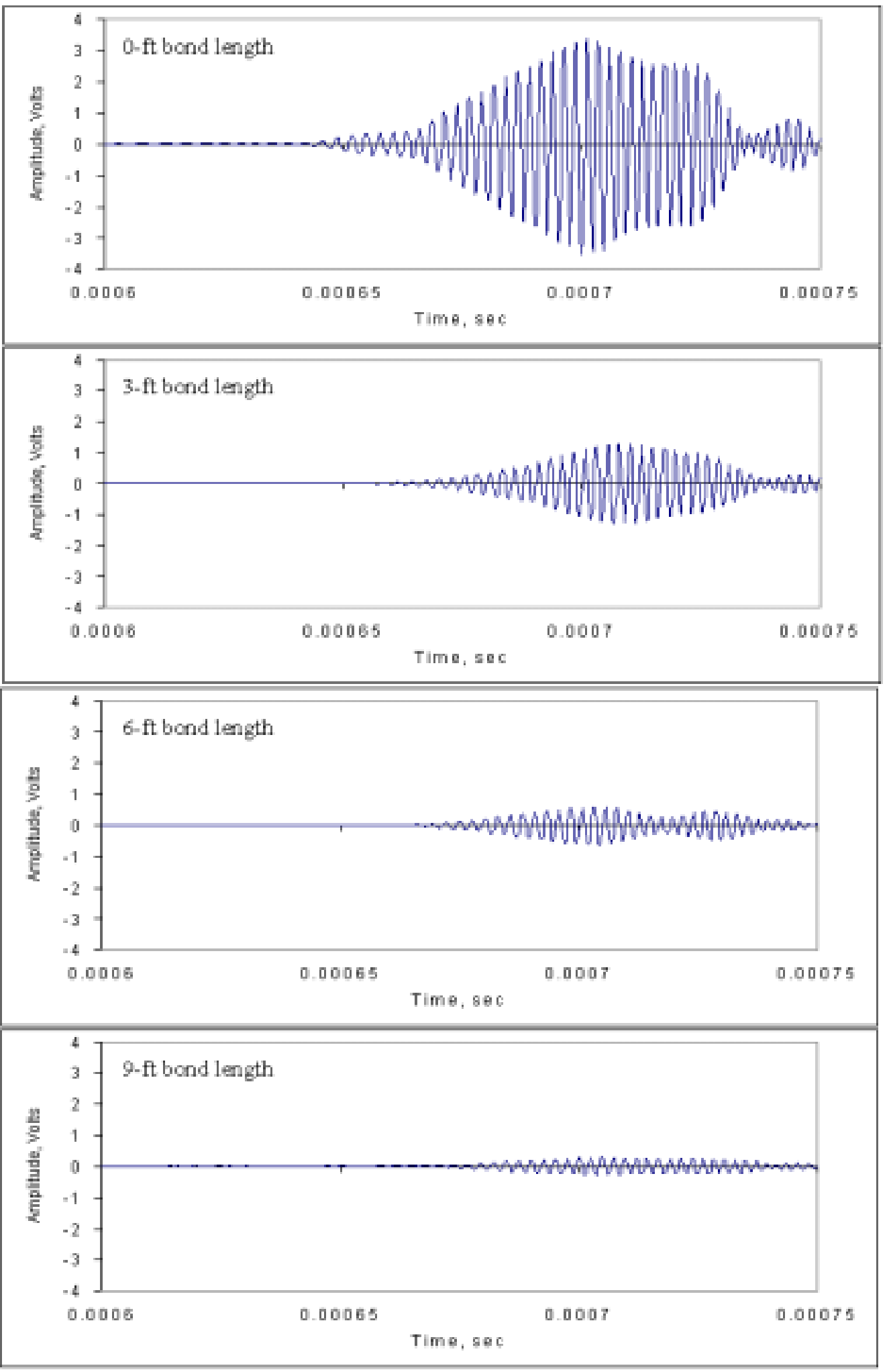

Figure 6.31 Recorded stress waves due to different bonding length between the seven-wire strand and concrete 


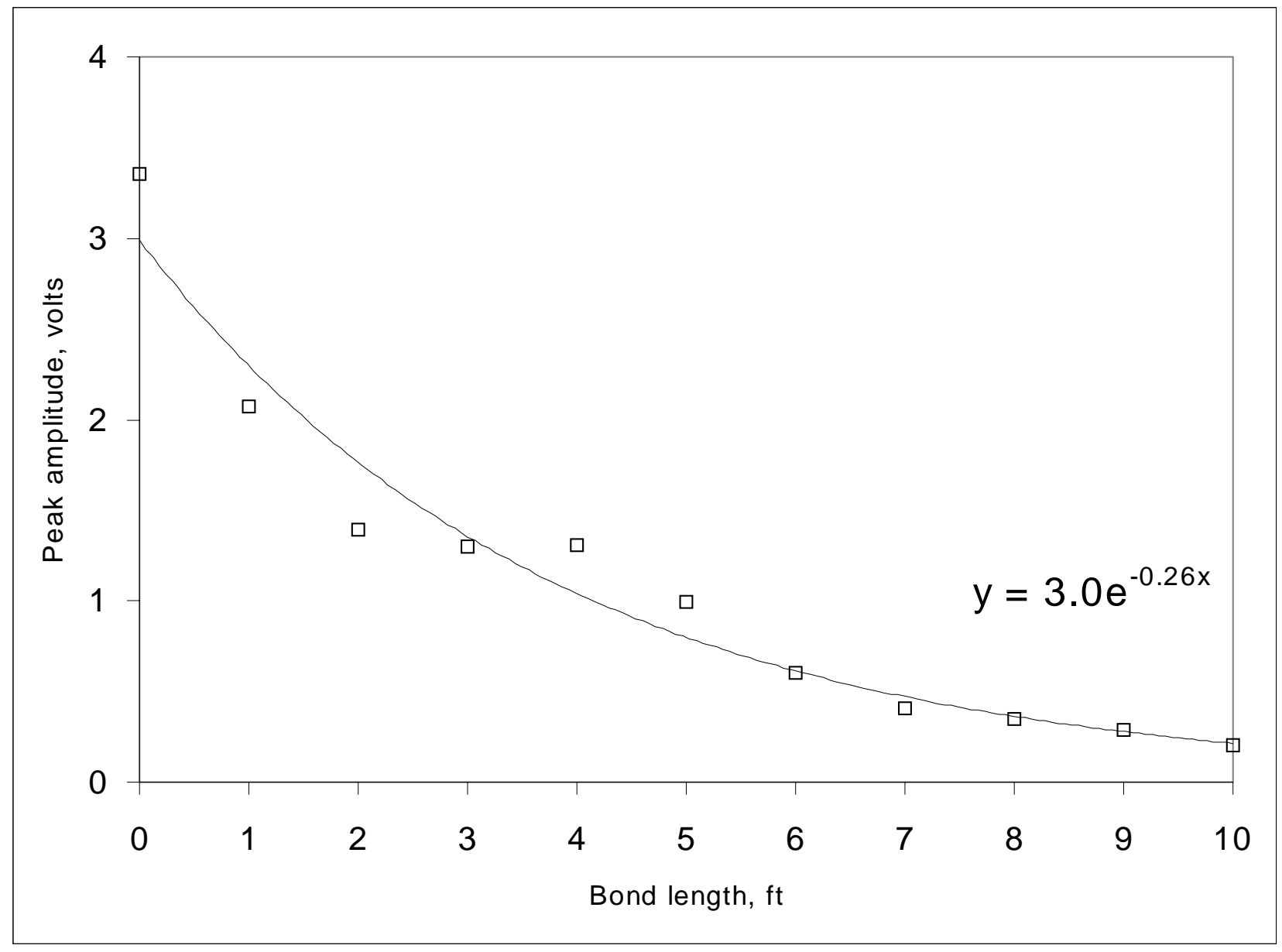

Figure 6.32 Attenuation of peak amplitudes of the stress waves due to bonding between the strand and concrete 


\section{CHAPTER 7}

\section{CONCLUSIONS AND RECOMMENDATIONS}

\subsection{ACOUSTIC EMISSION MONITORING OF REINFORCED CONCRETE STRUCTURES USING TWO-DIMENSIONAL SURFACE WAVEGUIDE}

\section{Conclusions}

This study presents an innovative AE technique by introducing a two-dimensional acoustic surface waveguide system to enhance the ability to detect and to locate AE signals in concrete structures. The waveguide mesh made of steel wires was attached on the surface of a reinforced concrete floor slab. High transmission efficiencies of AE signals were experimentally obtained for the epoxy joints, which were used to attach the waveguides with the concrete surface. Experimental results confirm that the AE signals propagating in a surface waveguide have less attenuation than that in concrete so that the AE monitoring area can be enlarged without applying a large number of sensors and monitoring devices. A multi-layer neural network system was successfully employed to locate the AE sources in concrete. The neural network system was trained using four data sets of AE parameters and the source locations. A testing data set was used and effectively verified the ability of the neural network in locating the AE sources. Satisfactory prediction results from the neural network were obtained. The results of using the two-dimensional surface waveguide together with the neural network system shows that the system has ability to efficiently detect and locate $\mathrm{AE}$ activities in the concrete structures. 


\section{$\underline{\text { Recommendations }}$}

Proper design and construction of the waveguide system on the concrete surface can improve the transmission efficiency and prediction accuracy of the whole system. In practice, the joint material needs to be carefully selected so as to provide strong bonding between the waveguides mesh and the concrete surface in different environments (e.g. extreme temperature, vibrations). Construction process of the joints needs to be considered so that the joints of each type are identical. This would improve the accuracy of the source location identification. Development of a portable waveguide system is strongly recommended so that less amount of time is spent for the installation of the system. In addition, training of the neural network is required only once for each waveguide system. Therefore, an on-site calibration may not be necessary.

A further step of this study is recommended to evaluate the application of the surface waveguide system on a concrete structure where actual cracks are active. Applying the acousto-ultrasonic method to the proposed waveguide system is also recommended. In this method, ultrasonic pulses can be generated as artificial AE signals at different locations on the concrete surface and be captured by the surface waveguide. Pattern of the detected AE signals can then be used to identify the damage of the structure through a comparative study with an undamaged model simulation. This would provide an alternative way to inspect damages in structures made of concrete as well as other high attenuation materials, such as fiber reinforced composite plates and FRP vessels.

Another recommended research work is the AE signal characterization. Despite the complex nature of AE signals, the study of relationships between AE parameters and failure 
mechanics of concrete structures is still needed. The future work is to develop the method for $\mathrm{AE}$ signal interpretation in the waveguide system by combining the wave propagation theory of waveguides with the understanding of relationships between AE characteristics and failure behavior of concrete.

\subsection{TENSILE FORCE MEASUREMENT OF SEVEN-WIRE PRESTRESSING STRANDS USING STRESS WAVES}

\section{Conclusions}

This study presents the development of a nondestructive, stress wave technique in evaluating prestress forces in seven-wire prestressing strands, which are widely used in various types of prestressed concrete structures. The $1 / 2$-inch diameter seven-wire strands of Grade 250 and Grade 270 were tested under varying tensile forces. Numerical analyses were conducted by accounting for the acoustoelasticity effect and dispersion of waves to calculate the prestress levels in the strands. This analysis provides a successful description of the behavior of a longitudinal transient wave traveling through a long, prestressed, circular strand. Experimental results show that the relative change of tensile forces in the strand can be evaluated by measuring time shift of any particular peak amplitude of the recorded waveforms. The time shift measurement were conducted entirely in time domain of the waveforms. Numerical results show that different frequency components of a traveling wave propagate with different velocities. The tension level in the strand also affects the wave speed of each particular frequency. By using the Wigner-Ville transform as a signal processing tool, both time and frequency information of the recorded waveforms can be 
obtained simultaneously. Therefore, the arrival time (or velocity) of each frequency component of the waveforms can be identified. The arrival time of this particular frequency was used to back-calculate the tensile force in the strand. Change of the stress wave velocity at different stress levels was evaluated and accurate measurement results from this technique were obtained. In addition, material constants of the strands (Lame's and Murnaghan's elastic constants) can be indirectly determined based on results obtained from at least two tension levels. The material constants obtained using the indirect method are only the approximation of the actual material properties of the prestressing strand. For some other applications, these material constants may not be valid. In this technique, these constants were used to predict the group velocity of the waves at other tension levels and accurate predictions were obtained. It is observed from field measurements that the generated stress wave impulses can propagate a very far distance up to about $275 \mathrm{ft}$ in the uncoated posttensioning strands. Attenuation of stress wave signals becomes more severe for the posttensioned monostrand system where each strand was coated by lubricant and was sheathed by protection sleeves. Severe attenuation was also observed in the pretensioning system where the energy of the wave was attenuated in a much faster rate due to the presence of concrete. Using the same set of instrument, monitoring ranges of this technique based on results due to different length of strands were estimated to be $295 \mathrm{ft}$ for post-tensioning system and $21 \mathrm{ft}$ for pretensioning system. For overall performance, the developed technique can provide an efficient nondestructive measurement of tension levels in the sevenwire prestressing strands. 


\section{$\underline{\text { Recommendations }}$}

It was shown that each of the material constants of the strands $(\rho, \lambda, \mu, 1, \mathrm{~m}$, and $\mathrm{n})$ unequally affects the predicted value of the tensile force. Due to limitations of the instruments used in this study, these constants were numerically adjusted using the trial-anderror method in order to provide the best fit to experimental results. In practical cases, strands' material properties may vary from one manufacturer to another depending on their chemical compositions, manufacturing processes, and quality controls. Since there are many material properties and each of which is subjected to several types of variations, numerical calculations of the tensile force in the strand are complicated. However, results from numerical analyses show a simple relationship between the relative change in group velocities and stress levels in the strand. Using the material properties of the $1 / 2$-inch sevenwire prestressing strand of Grade 270 (Table 6.1), the relative change in group velocity of each particular frequency is linearly proportional to the stress level in the strand (Figure 7.1). This relationship can be expressed as:

$$
\left(\frac{C_{g}^{\sigma}(\omega)-C_{g}^{o}(\omega)}{C_{g}^{o}(\omega)}\right)=K(\omega) \cdot \sigma
$$

where, $C_{g}{ }^{\sigma}(\omega)$ is the group velocity of the frequency component $\omega$ of the wave due to applied tensile stress $\sigma . \quad C_{g}{ }^{o}(\omega)$ is the group velocity of the frequency component $\omega$ of the wave due to zero stress in the strand. The slope of each line (Figure 7.1), representing each frequency $\omega$, is designated as $K(\omega)$. Hence, all material constants of the strand are lumped into a single value of $K(\omega)$. The relationship between $K(\omega)$ and $\omega$ is plotted in Figure 7.2. In 
order to establish the $K(\omega)$ value, measurements of group velocities due to two tensile force levels are needed. As shown in Figure 7.3, dispersion curves due to 0 kips and 30 kips tensile forces are illustrated. From these curves, the relative change in group velocity at a known stress level can be used to calculate the $K(\omega)$ value for each frequency. Database of $K(\omega)$ and $C_{g}{ }^{o}(\omega)$ values should be constructed for each type of strands in the future. Thus, the measurement of a group velocity, $C_{g}{ }^{\sigma}(\omega)$, of only one particular frequency is required for a strand used in an existing structure. Tolerance of this measurement technique should be determined. Accuracy of this technique may also be affected by other factors such as coating of strand and temperature at the time of measurement. Investigation of these factors should also be conducted in the future.

As described in previous chapters, analysis of the recorded stress wave signals is a complicated task and the current data acquisition system (MISTRAS) is not able to analyze the waveforms in time-frequency plane. Thus, waveform analyses using WVT have to be conducted separately and this becomes a time-consuming process. For this reason, there is a need for a computer program, which can retrieve and analyze waveform data directly from the data acquisition system. The developed computer program should be able to give an instant value of tensile forces right after the stress wave signals were recorded. 


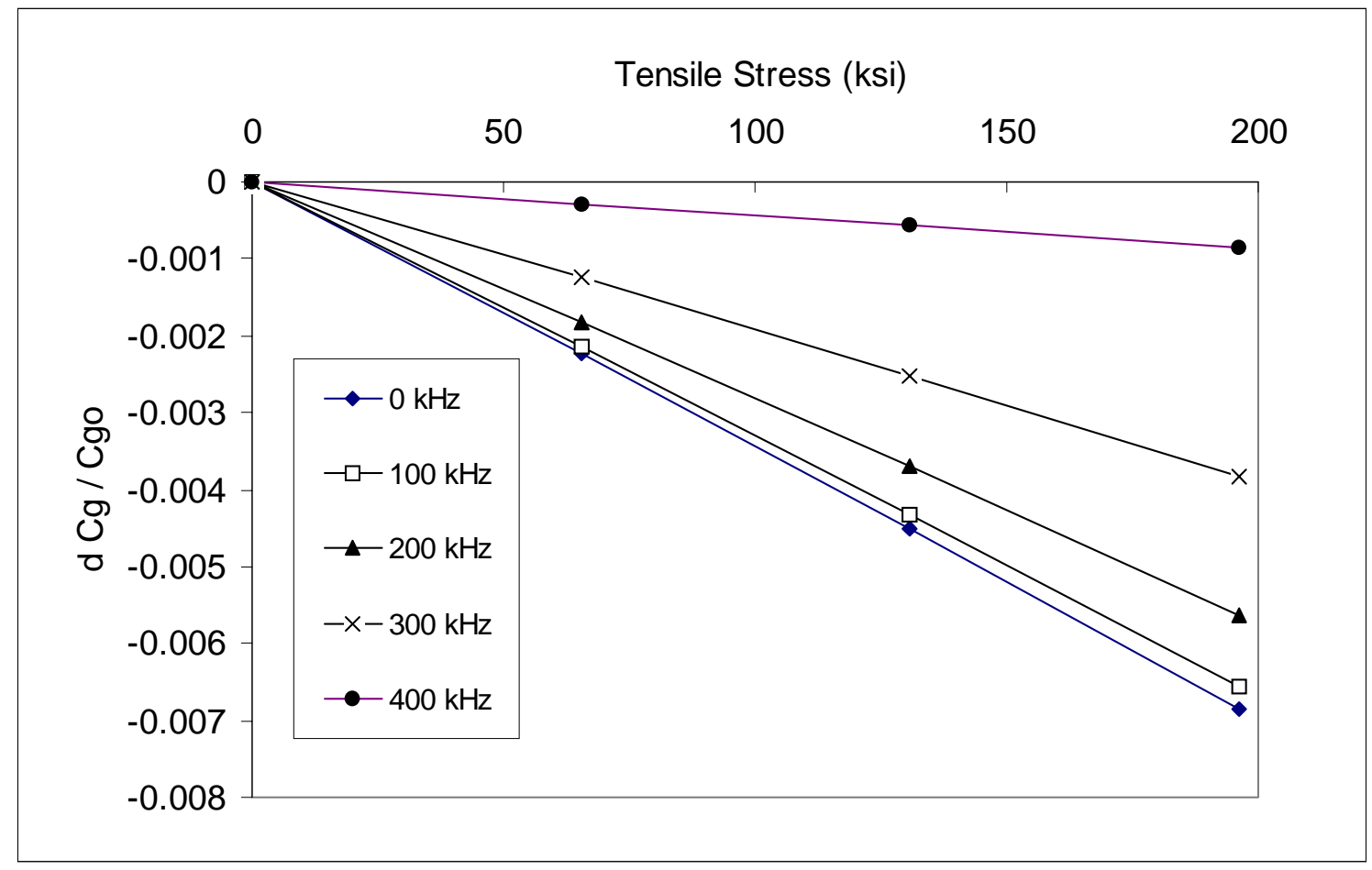

Figure 7.1 Relative change in group velocity due to tensile stress in the $1 / 2$-inch sevenwire prestressing strand of Grade 270. 


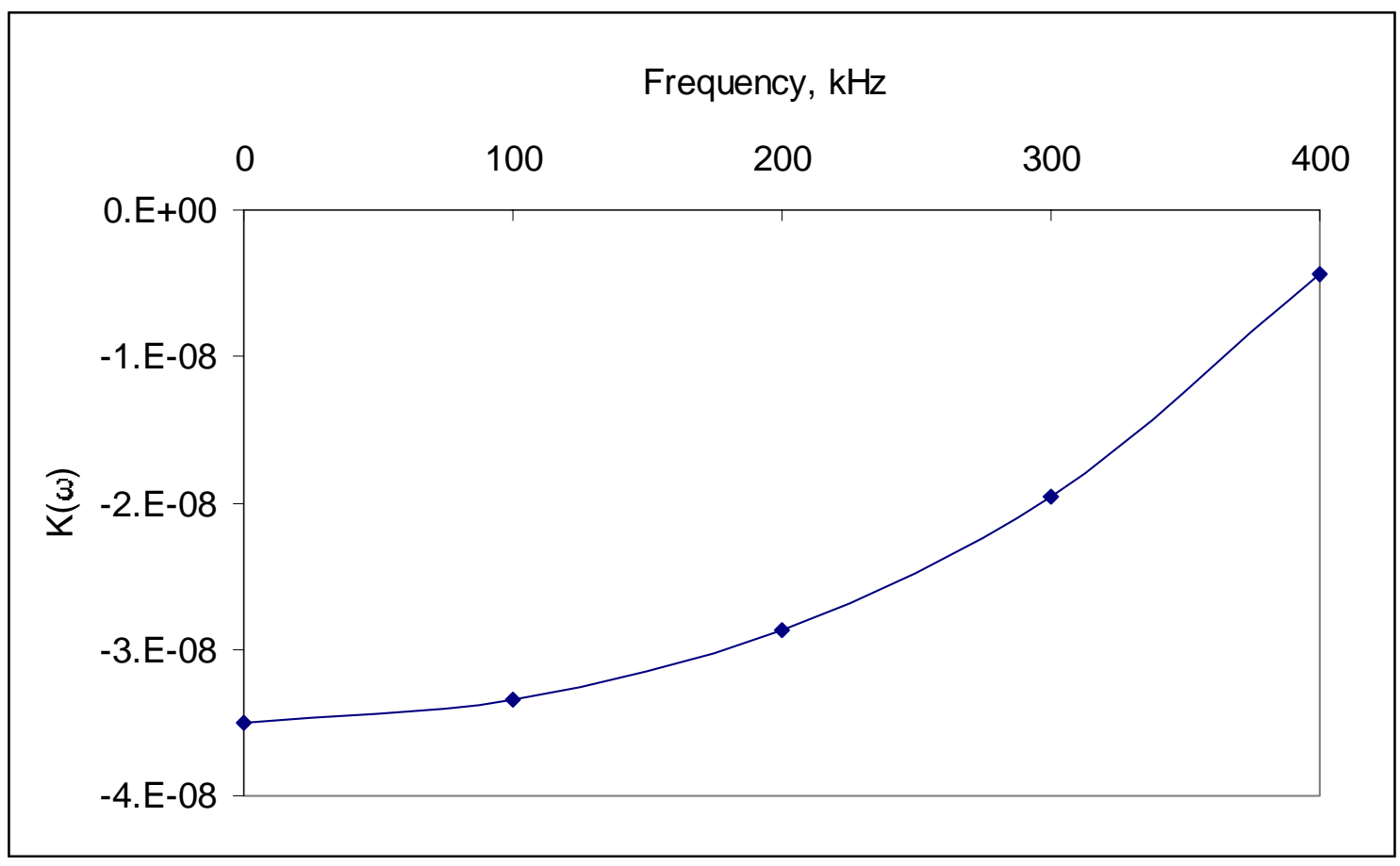

Figure 7.2 Relationship between $\mathrm{K}(\omega)$ and propagation frequency of a stress wave 


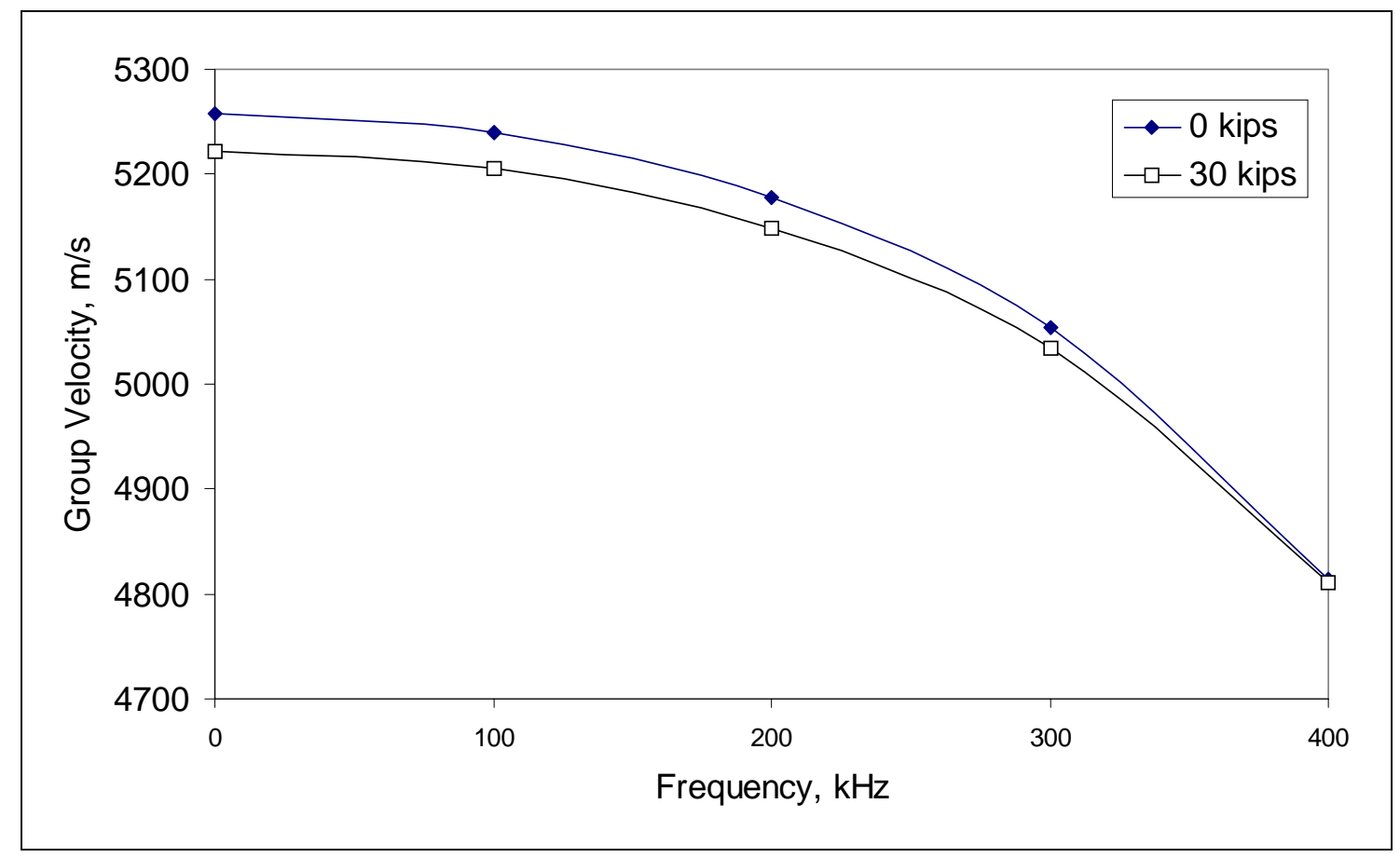

Figure 7.3 Dispersion curves due to 0 kips and 30 kips tensile force in the seven-wire strand of Grade 270 . 


\section{REFERENCES}

-Allison, S.G., J.S. Heyman, and K. Salama, " Ultrasonic Measurement of Residual Deformation Stress in Thin Metal Plates Using Surface Acoustic Waves," IEEE Ultrasonic Symposium, 1983.

-Allison, S.G., and C.G. Clendenin, "Ultrasonic Pulsed Phase Locked Loop Interferometer for Bolt Load Measurements," $35^{\text {th }}$ International Instrumentation Symposium, Instrument Society of America, May, 1989, p.202.

-Achenbach, J.D., Wave Propagation in Elastic Solids, North-Holland Publishing, 1973.

-Allen, D.R., and C.M. Sayers, “ The Measurement of Residual Stress in Textures Steel Using an Ultrasonics Velocity Combination Technique," Ultrasonics, 1984, Vol.22, p.179.

-Auger, F., P. Flandrin, P. Goncalves, and O. Lemoine, "Time-Frequency Toolbox," Centre National de la Recherche Scientifique, France, 1995.

-Bickford, J.H., "Using Ultrasonics to Measure the Residual Tension in Bolts, " Experimental Techniques, 1988, Vol.12, p.3s.

-Binka, J., and W. Sachse, "Application of Ultrasonic-pulse-spectroscopy Measurements to Experimental Stress Analysis,” Experimental Mechanics, 1976, Vol.16, p.448.

-Boving, K.G., NDE Handbook: Non-destructive Examination Methods for Conditioning Monotoring, 1989.

-Bray, D.E., and D. McBride, "Nondestructive Testing Techniques," John Wiley \& Sons, 1992.

-Chen, H.L., Y. He, and H.V.S. GangaRao, " Measurement of Prestress Force in the Rods of Stressed Timber Bridges Using Stress Waves, " Materials Evaluation, 1998, Vol.56, No.8, p.977.

-Chen, H.L., C.T. Cheng, and S.E. Chen, "Determination of Fracture Parameters of Mortar and Concrete Beams by Using Acoustic Emission," Materials Evaluation, Vol.50, No.7, July 1992, pp. 273-281.

-Chen, H.L., and C.L. Chen, "Applying Neural Network to Acoustic Emission Signal Processing," Proceedings in 4th International Symposium on Acoustic Emission From Composite Materials, Seattle, WA, 1992, pp. 273-281.

-Chen, H.L., Y. He, and M. Superfesky, "Acoustic Surface Waveguides for Acoustic Emission Monitoring of Fiber-Reinforced Plastic Structures," Materials Evaluation, Vol.52, No.9, Sep. 1994, pp. 1112-1116. 
-Chen, H.L. and Y. He, "Analysis of Acoustic Surface Waveguides for Nondestructive Evaluation of Concrete Structures," Engineering Mechanics, Proceedings of the $10^{\text {th }}$ Conference, ASCE-EMD, Boulder, Colorado, May 1995.

-Chen, H. L., and Y. He, "Analysis of Liquid-core Cylindrical Acoustic Waveguides," Journal of Engineering Mechanics, ASCE, Vol.122, No.1, Jan. 1996, pp. 1-9.

-Chen, H. L. and Y. He, "Analysis of an Acoustic Waveguide for Acoustic Emission Monitoring of Concrete Beams," Journal of Engineering Mechanics, ASCE, Vol.127, No.1, January 2001, pp. 1-10.

-Chen, C.L., "Training the Multi-layer Neural Nets by Setting the Initial Weights," Ph.D. Dissertation, West Virginia University, WV, 1992.

-Clark, A.V., and J.C. Moulder, "Residual Stress Determination in Aluminum Using Electromagnetic Acoustic Transducers," Ultrasonics, Vol.23, 1985, p. 253.

-Cun, Y.L., "Generalization and Network Design Strategies: Connectionism in Perspective", North Holland, Zurich, Switzerland, 1989, pp.143-155.

-Cun, Y.L., L.D. Jackel, B. Boser, J.S. Denker, H.P. Graf, I. Guyon, D. Henderson, R.E. Howard, and W. Hubbard, "Handwritten Digit Recognition: Applications of Neural Network Chips and Automatic Learning," Comm. Magazine, November 1989, pp. 41-46.

-Cun, Y.L., and D.S. Touretzky, "Applying Neural Network Technologies," $8^{\text {th }}$ National Conference on Artificial Intelligence, Tutoral MA1, 1990.

-Denker, J.S. et. al., "Neural Network Recognizer for Handwritten Zip Code Digits," Advances in Neural Information Processing Systems, Morgan Kaufmann Publisher, San Mateo, CA, 1989, pp.323-331.

-Desch, C.H., D.O. Sproule, and W.J. Dawson, "The Detection of Cracks in Steel by Means of Supersonic Waves," J. Iron Steel Inst., Vol.153, 1946, pp. 319-352.

-Dike, J.J., and G.C. Johnson, “ Residual Stress Determination using Acousto-elasticity," Journal of Applied Mechanics, Vol.57, March 1990, pp.12-17.

-Doyle, P.A., and C.M. Scala, "Crack Depth Measurement by Ultrasonics: A Review," Ultrasonics, Vol.16, 1978, pp.164-170.

-Dunegan Testing Group, “Acoustic Emission Testing of Buried Pipeline," Dunegan Testing \& Inspection, Houston, TX, 1989.

-Dunegan Corporation, "Acoustic Emission Monitoring of Electroslag and Butt Welds on Dunbar Bridge, West Virginia," Final Report: West Virginia DOH Project 64, 1985. 
-Egle, D.M., and D.E. Bray, "Measurement of Acoustoelastic and Third-order Elastic Constants for Rail Steel," The Journal of the Acoustical Society of America, 1976, Vol.60, No.3, p.741.

-Firestone, F.A., Flaw Detecting Device and Measuring Instrument, US Patent, 2, 280, 226, 1942; and 2,280, 130, 1940.

-Folk, R., G. Fox, C.A. Shook, and C.W. Curtis, “ Elastic Strain Produced by Sudden Application of Pressure to One End of a Cylindrical Bar: I. Theory, " The Journal of the Acoustical Society of America, 1958, Vol.30, p.552.

-Gong, Z., E.O. Nyborg, and G. Oommen, "Acoustic Emission Monitoring of Steel Railroad Bridges,” Material Evaluation, Vol.50, No.7, 1992, pp. 883-887.

-Gorman, R.P., and T.J. Sejnowski, "Analysis of Hidden Units in a Layered Network Trained to Classify Sonar Targets," Neural Networks, Vol.1, 1988, pp. 75-79.

-Grabec, I., and W.Sachse, "Application of an Intelligent Signal Processing System to Acoustic Emission Analysis," The Journal of the Acoustical Society of America. V85, Mar. 1989, pp. 1126- 1135.

-Griffith, D.W., J.G. Gonzalez, and G.R. Arce, "Robust Time Frequency Representations for Signals in Alpha-Stable Noise Using Fractional Lower-Order Statistics," Proceedings of the 1997 IEEE Signal Processing Workshop on Higher-Order Statistics by the IEEE Signal Processing Society, July, 1997.

-Hawkins, N.M., W.M. McCabe, and Y. Nobuta, "Use of Acoustic Emission to Detect Debonding of Reinforcing Bars in Concrete," Progress in Acoustic Emission IV, The Japanese Society for NDT, 1988, pp. 342-349.

-He, Y., "Acoustic Emission Monitoring of Concrete Structures Using Waveguides," Ph.D. Dissertation, West Virginia University, 1996.

-Hearn, S.W., and C.K. Shield, "Acoustic Emission Monitoring as a Nondestructive Testing Technique in Reinforced Concrete,” ACI Material Journal, November, 1997, pp. 510-519.

-Heyman, J.S., and E.J. Chern, “ Ultrasonic Measurement of Axial Stress," Journal of Testing and Evaluation, September, 1982.

-Hopwood, T., "Acoustic Emission, Fatigue, and Crack Propagation," Report No.457., Kentucky Bureau of Highways, 1976.

-Hornik, K., “Approximation Capabilities of Multi-layer Feed-forward Networks," Neural Networks, Vol.4, No.2, 1991, pp. 251-257. 
-Hughes, D.S., and J.L. Kelly, “ Second-Order Elastic Deformation of Solids,” Physical Review, 1953, Vol.95, No.5, p.1145.

-Hutton, P.H. and J.R. Skorpik, "Acoustic Emission Methods for Flaw Detection in Steel Highway Bridges,” Federal Highway Administration Report No. FHWA-RD-78-98, 1978.

-Johnson, G.C., A.C. Holt, and B. Cunningham, "An Ultrasonic Method for Determining Axial Stress in Bolts," Journal of Testing and Evaluation, 1986, Vol.14, p.253.

-Joshi, S.G., and R.G. Pathare, "Ultrasonic Instrument for Measuring Bolt Stress," Ultrasonics, 1984, Vol.22, p.270.

-Kimura, S., I. Adachi, Y. Hironaka, and M. Ohtsu, “ AE Evaluation of Concrete Structures,” Progress in Acoustic Emission IV, The Japanese Society for NDI, 1988, pp. 350-358.

-Krautkramer, J., and H. Krautkramer, Ultrasonic Testing of Materials, $3^{\text {rd }}$ edition, SpringerVerlag, 1983.

-Li, Z.F., A. Zdunek, E. Landis, and S.P. Shah, "Application of Acoustic Emission Technique to Detection of Reinforcing Steel Corrosion in Concrete," ACI Material Journal, January 1998, pp. 68-76.

-Lim, M.K., and T.K. Koo, “Acoustic Emission from Reinforced Concrete Beams,” Mag. of Concrete Res., Vol.41, 1989, pp. 229-234.

-Lovass, S., " Acoustic Emission of Offshore Structures: Attenuation; Noise; Crack Monitoring," Journal of Acoustic Emission, April, 1985, p.161.

-Lu, W.Y., and C.S. Man, "Measurement of Stress Based upon Universal Relations in Acoustoelasticity," Experimental Mechanics, Vol.29, 1989, p. 109.

-Machida, S., and A.J. Durelli, "Response of a Strand to Axial and Torsional Displacements," Journal of Mechanical Engineering Science, 1973, Vol.15, No. 4, p.241.

-Maji, A., and S.P. Shah, "Process Zone and Acoustic-Emission Measurement in Concrete," Experimental Mechanics, Vol.28, No.1, Mar. 1988, pp. 27-33.

-Maliszkiewicz, P.A., " Acoustic Emission Possibilities of Application to Tests of Concrete Structures," $2^{\text {nd }}$ Workshop on Bridge Engineering Research, 1990, pp. 99-102.

-Matsuyama, K., T. Fujiwara, A. Ishibashi, and M. Ohtsu, “ Field Application of Acoustic Emission for Diagnostic of Structural Deterioration of Concrete," Journal of Acoustic Emission, Vol.11, No.4, 1993, pp. 65-73.

-Meyer, Y., "Wavelets: Algorithms and Applications", Society for Industrial and Applied Mathematics, Philadelphia, PA, 1993. 
-Miller, R.K., and P. McIntire, "Nondestructive Testing Handbook: Vol. 5 Acoustic Emission Testing," $2^{\text {nd }}$ edition, American Society for Nondestructive Testing, 1987.

-Mulhhauser, German Patent 569, 598, 1933.

-Mullins, L., Evolution of NDT, Progr. Appl. Mater. Res., Vol.5, 1964, pp.205-212.

-Murnaghan, F.D., Finite Deformation of an Elastic Solid, John Wiley \& Sons, New York, 1951.

-Ohtsu, M., “ Determination of Crack Orientation by Acoustic Emission," Materials Evaluation, Vol.45, No.9, 1986, pp. 1070-1075.

-Ohtsu, M., "Acoustic Emission Characteristics in Concrete and Diagnostic Application," Journal of Acoustic Emission, Vol.6, No.2, 1987, pp. 99-108.

-Ohtsu, M., "Crack Propagation in Concrete: Linear Elastic Fracture Mechanics and Boundary Element Method," Theoretical and Applied Fracture Mechanics, Vol.9, No.1, 1988, pp. 55-60.

-Ohtsu, M., T. Okamoto, and S. Yuyama, “Moment Tensor Analysis of Acoustic Emission for Cracking Mechanisms in Concrete,” ACI Structural Journal, March 1998, pp. 87-95.

-Olivito, R.S., and L. Surace, “ The Damage Assessment of Concrete Structures by TimeFrequency Distributions," Experimental Mechanics, Vol.37, No.3, 1997, pp. 355-359.

-Ouyang, C.S., E. Landis, and S.P. Shah, "Damage Assessment in Concrete Using Quantitative Acoustic Emission," Journal of Engineering Mechanics Division, Vol.117, No.11, Nov. 1991, pp. 2681-2698.

-LOCAN-AT User's Manual, Physical Acoustics Corporation, Princeton, NJ 1988.

-Pao, Y.H., W.Sachse, and H. Fukuoka, "Acoustoelasticity and Ultrasonic Measurements of Residual Stresses," Physical Acoustics, edited by W.P. Mason and R.N. Thurston, 1984, Vol.XVII, p.61.

-Pollock, A.A., "Acoustic Emission Inspection," Physical Acoustic Corporation, Princeton, NJ, 1989.

-Prabhu, D.R. et. al., "Disbond Detection Through Ultrasonic Signal Classification Using an Artificial Neural Network," Analytical Services and Materials, MS231, NASA Langley Research Center, Hampton, VA, 1991. 
-Rumelhart, D.E., G.E.Hinton, and R.J. Williams, "Learning Internal Representations by Error Propagation," Parallel Distributed Processing: Explorations in the Microstructure of Cognition, Vol. 1, MIT press, Cambridge, MA, 1986, pp. 318-362.

-Sejnowski T., and C. Rosenberg, "NETtalk: a Parallel Network that Learns to Read Aloud," Johns Hopkins University Tech. Rep. JHU/EECS-86/01, 1986.

-Sokolov, S., "Propagation of Ultra-acoustic Oscillations in Various Bodies," Elekt. Nach Tech., Vol.6, 1929, pp.450-461.

-Sokolov, S., Ultrasonic Methods for Determining Internal Flaws in Metal Objects, Zavod. Lab., Vol.1, 1935, pp.1468-1473.

-Uomoto, T., "Application of Acoustic Emission to the Field of Concrete Engineering," Journal of Acoustic Emission, Vol.6, No.3, 1987, pp. 137-144.

-Waibel, A., T. Hanazawa, G. Hinton, K. Shikano, and K.J. Lang, "Phone Recognition Using Time-Delay Neural Networks," IEEE Trans ASSP, Vol.37, No.3, March 1989.

-Wood, R.W., and A.L. Loomis, Physical Review, Abstract, Vol.29, 1927, p.273.

-Woodward, R.J., “ Cracks in Concrete Bridge,” Concrete, July 1983, pp.40-43.

-Yoon, D.J., W.J. Weiss, and S. Shah, “Assessing Damage in Corroded Reinforced Concrete Using Acoustic Emission”, Journal of Engineering Mechanics, Vol. 126, No. 3, March 2000, p.273.

-Yuhas, B.P., M.H. Goldstein, and T.J. Sejnowski, "Integration of Acoustic and Visual Speech Using Neural Networks,” IEEE Comm. Mag., November 1989, pp.65-71.

-Yuyama, S., T. Imanaka, and M. Ohtsu, "Quantitative Evaluation of Microfracture due to Disbonding by Waveform Analysis of Acoustic Emission," Journal of the Acoustical Society of America, Vol.83, No.3, 1994, pp. 976-983.

-Yuyama, S., T. Okamoto, and S. Nagataki, " Acoustic Emission Evaluation of Structural Integrity in Repaired Reinforced Concrete Beams," Materials Evaluation, Vol.52, No.1, 1994, pp. 86-90.

-Yuyama, S., T. Okamoto, and M. Shigeishi, “Acoustic Emission Generated in Corners of Reinforced Concrete Rigid Frame Under Cyclic Loading," Materials Evaluation, Vol.53, No.3, 1995a, pp. 409-412.

-Yuyama, S., T. Okamoto, and M. Shigeishi, " Quantitative Evaluation and Visualization of Cracking Process in Reinforced Concrete by a Moment Tensor Analysis of Acoustic Emission," Materials Evaluation, Vol.53, No.6, 1995b, pp. 751-756. 


\section{APPENDIX A: WAVE MOTIONS IN A SURFACE WAVEGUIDE}

This section discusses the wave motions and their dispersion in a surface waveguide. In this study, the surface waveguides, made of steel wires, can be modeled as a solid, circular waveguide with a free outer boundary condition. The waveguides of this criteria have been extensively studied (Achenbach, 1973). Literature shows that there are different dispersive relationships for different propagation modes. Details of the exact theory for this type of waveguides can be found in the above literature. In addition, a simplified approach called the one-dimensional approximation of guided wave motions was also introduced. The validity of one-dimensional approximation is that the wave motions considered must be in low frequency range such that the wavelength of the considered wave motions is ten times greater than the radius of the waveguide. In this study, the proposed surface waveguide is made of steel wire with $1 \mathrm{~mm}$ diameter and the major frequency content of typical AE signals is from $100 \mathrm{kHz}$ to $300 \mathrm{kHz}$. Considering a wave speed of 5,000 m/s, (longitudinal wave speed in steel), the wavelength for $300 \mathrm{kHz}$ is about $16 \mathrm{~mm}$. This satisfies the above requirement of applying one-dimensional approximation.

The basic assumption of one-dimensional approximation is that there is no relative displacement within a cross section. In other words, every point at the same cross section of the waveguide has the same displacement for longitudinal and flexural displacements and the same degree of rotation along the waveguide axis for torsional displacement. Hence, there are three guided propagation modes of wave motions in the waveguides: longitudinal, flexural, or torsional mode. The differential equations for each of these propagation modes are: 
for longitudinal wave motions:

$$
E \cdot A \frac{\partial^{2} u}{\partial x^{2}}-\rho \cdot A \frac{\partial^{2} u}{\partial t^{2}}=0
$$

for flexural wave motions:

$$
E \cdot I \frac{\partial^{4} w}{\partial x^{4}}+\rho \cdot A \frac{\partial^{2} w}{\partial t^{2}}=0
$$

for torsional wave motions:

$$
\mu \cdot J \frac{\partial^{2} \theta}{\partial x^{2}}-\rho \cdot J \frac{\partial^{2} \theta}{\partial t^{2}}=0
$$

where, $w, u$, and $\theta$ are displacements in the transverse direction, the longitudinal direction, and rotation, respectively. Variables $E, \mu, \rho, A, I$, and $J$ are Young's modulus, shear modulus, mass density, cross-sectional area, moment of inertia, and rotational inertia of the steel wire, respectively.

The one-dimensional approximation of guided wave motions results in the following equations (Achenbach, 1973):

for longitudinal mode:

$$
u=A_{l} \cdot e^{-i \omega\left(\frac{x}{V_{l}}-t\right)}, V_{l}=\sqrt{\frac{E}{\rho}}
$$

for flexural mode:

$$
v=A_{f} \cdot e^{-i \omega\left(\frac{x}{V_{f}}-t\right)}, V_{f}=\sqrt{\omega}\left(\frac{E I}{\rho A}\right)^{1 / 4}
$$


for torsional mode:

$$
\theta=A_{t} \cdot e^{-i \omega\left(\frac{x}{V_{t}}-t\right)}, V_{t}=\sqrt{\frac{\mu}{\rho}}
$$

where, $V_{l}, V_{f}$, and $V_{t}$ are the propagation velocities for longitudinal mode, flexural mode, and torsional mode, respectively. $\mathrm{A}_{l}, \mathrm{~A}_{f}$, and $\mathrm{A}_{t}$ are constants. The above equations indicate that propagation velocities of longitudinal and flexural modes are not dispersive since they are not functions of frequency. According to the exact waveguide theory, wave motions in these two modes are also dispersive, but in low frequency range the dispersion is insignificant. On the other hand, the velocity of wave motions in flexural mode is proportional to the square root of the frequency, which implies a severe dispersion. The dispersion of wave motions in a waveguide is due to the fact that the group velocity, at which wave motions propagate, is a function of frequency. For wave motions with multi-frequency components (as in typical AE signals), dispersion of the signals in flexural mode causes the energy of wave motions to distribute over a longer distance as they propagate further along the waveguide. This results in an increase of duration, a decrease of peak amplitude of the signal, and the change of other AE characteristics. The reduction of peak amplitude reduces the sensivity of the AE monitoring. Wave motions in longitudinal mode appear to have less dispersion than those in flexural mode. Experimental verification of this phenomenon was reported by He (1996). Wave motions in torsional mode are not monitored in this study because they are difficult to be excited. Therefore, longitudinal mode is the only mode for signal transmission monitored in this study 


\section{APPENDIX B: THEORETICAL BACKGROUND ON THE FREQUENCY EQUATION}

The equation of wave motions in a homogeneous, isotropic, linearly elastic solid, in the absence of body force, is

$$
(\lambda+\mu) u_{j, j i}+\mu u_{i, j j}=\rho \ddot{u}_{i} \quad(i, j, k=1,2,3)
$$

where, $u$ is the displacement vector and is a function of coordinates and time, $\rho$ is mass density, $\lambda$ and $\mu$ are Lame's constants.

While studying a circular waveguide, it is necessary to use the cylindrical coordinates $(r, \theta, z)$. This coordinate system is shown in Figure B-1. Denoting the displacement components in the $r, \theta$, and $z$ directions by $u, v$, and $w$, respectively. In cylindrical coordinates, the displacement equations of motion of a wave propagating in the axial direction along a circular cylindrical rod can be written as

$$
\begin{aligned}
& \nabla^{2} u-\frac{u}{r^{2}}-\frac{2}{r^{2}} \frac{\partial v}{\partial \theta}+\frac{1}{1-2 v} \frac{\partial \Delta}{\partial r}=\frac{1}{C_{T}^{2}} \frac{\partial^{2} u}{\partial t^{2}} \\
& \nabla^{2} v-\frac{v}{r^{2}}+\frac{2}{r^{2}} \frac{\partial u}{\partial \theta}+\frac{1}{1-2 v} \frac{1}{r} \frac{\partial \Delta}{\partial \theta}=\frac{1}{C_{T}^{2}} \frac{\partial^{2} v}{\partial t^{2}} \\
& \nabla^{2} w+\frac{1}{1-2 v} \frac{\partial \Delta}{\partial z}=\frac{1}{C_{T}^{2}} \frac{\partial^{2} w}{\partial t^{2}}
\end{aligned}
$$


where $v$ is the Poisson's ratio, $\nabla^{2}$ is the Laplacian

$$
\nabla^{2}=\frac{\partial^{2}}{\partial r^{2}}+\frac{1}{r} \frac{\partial}{\partial r}+\frac{1}{r^{2}} \frac{\partial^{2}}{\partial \theta^{2}}+\frac{\partial^{2}}{\partial z^{2}}
$$

and the dilatation $\Delta$ is defined as

$$
\Delta=\frac{\partial u}{\partial r}+\frac{1}{r}\left(\frac{\partial v}{\partial \theta}+u\right)+\frac{\partial w}{\partial z}
$$

The stress-strain relations are

$$
\begin{aligned}
\tau_{r} & =\lambda \Delta+2 \mu \frac{\partial u}{\partial r} \\
\tau_{r \theta} & =\mu\left[\frac{1}{r}\left(\frac{\partial u}{\partial \theta}-v\right)+\frac{\partial v}{\partial r}\right] \\
\tau_{r z} & =\mu\left(\frac{\partial u}{\partial z}+\frac{\partial w}{\partial r}\right) .
\end{aligned}
$$

The general solution of equation (B-1) can be expressed in terms of a scalar potential $\Phi$ and vector potential $\psi$ as

$$
\underline{\mathbf{u}}=\underline{\nabla} \underline{\Phi}+\underline{\nabla} \times \underline{\Psi}
$$

The displacement components can be written in terms of displacement potentials as

$$
\begin{aligned}
& u=\frac{\partial \Phi}{\partial r}+\frac{1}{r} \frac{\partial \psi_{z}}{\partial \theta}-\frac{\partial \psi_{\theta}}{\partial z} \\
& v=\frac{1}{r} \frac{\partial \Phi}{\partial \theta}+\frac{\partial \psi_{r}}{\partial z}-\frac{\partial \psi_{z}}{\partial r}
\end{aligned}
$$




$$
w=\frac{\partial \Phi}{\partial z}+\frac{1}{r} \frac{\partial\left(\psi_{\theta} r\right)}{\partial r}-\frac{1}{r} \frac{\partial \psi_{r}}{\partial \theta}
$$

Longitudinal waves are axially symmetric waves characterized by the presence of displacement components in the radial and axial directions. The displacement potentials can be written as

$$
\begin{aligned}
& \Phi=A J_{0}(p r) e^{[i(k z-\omega t)]} \\
& \psi_{\theta}=C J_{1}(q r) e^{[i(k z-\omega t)]}
\end{aligned}
$$

where $\quad p^{2}=\frac{\omega^{2}}{C_{L}^{2}}-k^{2} \quad$ and $q^{2}=\frac{\omega^{2}}{C_{T}^{2}}-k^{2}$

$\omega$ is the angular frequency and $k$ is the wave number. $J_{0}\left(\right.$ ) and $J_{l}($ ) are the Bessel functions of the first kind of order 0 and 1.

The corresponding radial and axial displacements are obtained from equation (B-11) and equation (B-13) as

$$
\begin{aligned}
& u=\left\{-p A J_{1}(p r)-i k C J_{1}(q r)\right\} e^{[i(k z-\omega t)]} \\
& w=\left\{i k A J_{0}(p r)+q C J_{0}(q r)\right\} e^{[i(k z-\omega t)]}
\end{aligned}
$$

At the cylindrical surface $(r=a)$, the stresses must be zero. Substituting equation (B-16) and equation (B-17) into $\tau_{r}$, and setting the resulting expression equal to zero at $r=a$, 


$$
\left[-\frac{1}{2}\left(q^{2}-k^{2}\right) J_{0}(p a)+\frac{p}{a} J_{1}(p a)\right] A+\left[-i k q J_{0}(q a)+\frac{i k}{a} J_{1}(q a)\right] C=0 .
$$

The second equation comes from the condition that $\tau_{\mathrm{rz}}$ vanishes at $r=a$,

$$
\left[-2 i k p J_{1}(p a)\right] A-\left[\left(q^{2}-k^{2}\right) J_{1}(q a)\right] C=0 .
$$

In order to satisfy both equation (B-18) and equation (B-19), the determinant of the coefficients must vanish. The resulting frequency equation can be written as

$$
\frac{2 p}{a}\left(q^{2}+k^{2}\right) J_{1}(p a) J_{1}(q a)-\left(q^{2}-k^{2}\right)^{2} J_{0}(p a) J_{1}(q a)-4 k^{2} p q J_{1}(p a) J_{0}(q a)=0
$$

which is known as the Pochhammer's frequency equation. The frequency equation was used to estimate the group velocity of each frequency component of a stress wave propagating in center wires of the seven-wire prestressing strands. 


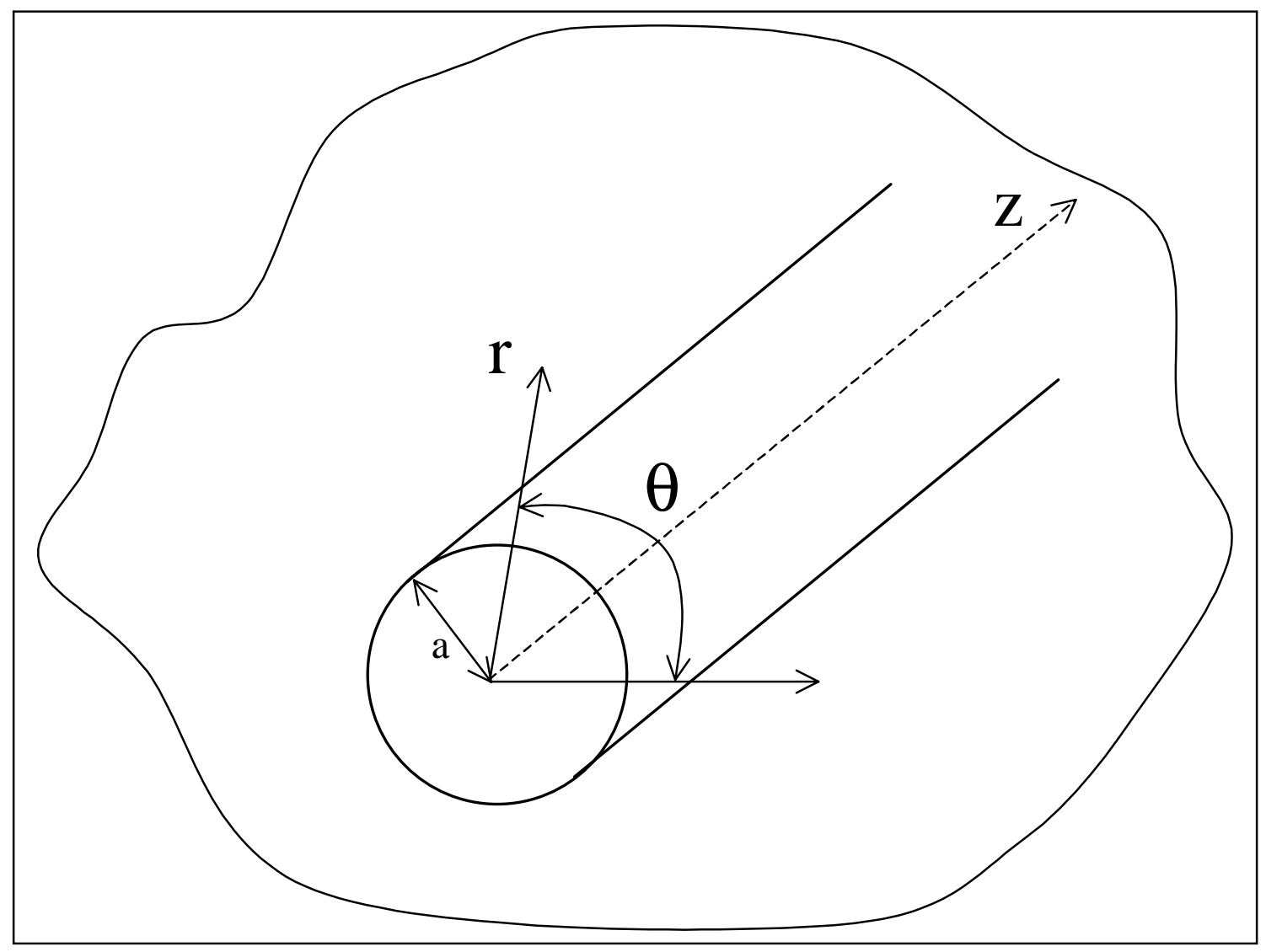

Figure B-1 Coordinate system of a circular waveguide 


\section{VITA}

Komwut Wissawapaisal was born in Bangkok, Thailand on August 8, 1971. In April 1989, he graduated with a high school diploma from Benjamarachutit School in Nakornsrithummarat province. He attended Chulalongkorn University and received his Bachelor of Engineering degree in May 1994. In January 1995, he was admitted to the graduate program in Civil Engineering at Texas A\&M University-Kingsville where he received his Master's degree in the same year. He entered the Ph.D. program in Civil and Environmental Engineering at West Virginia University in August 1996.

For the past five years, Mr. Wissawapaisal has been working as a graduate research assistant for the Constructed Facilities Center at West Virginia University. His research interest is in the area of nondestructive testing of reinforced and prestressed concrete structures. He is currently a candidate for the degree of Doctor of Philosophy in Civil Engineering.

\section{$\underline{\text { Publications }}$}

-Chen, H.L. and Wissawapaisal, K (2000)."Study of Acoustic Surface Waveguides on Reinforced Concrete Slabs", Journal of Nondestructive Evaluation, Vol. 19, No.4, pp.129140.

-Chen, H.L. and Wissawapaisal, K (2001)."Measurement of Tensile Forces in a Seven-Wire Prestressing Strand Using Stress Waves", Journal of Engineering Mechanics, ASCE, Vol.127, No.6, pp.599-606.

-Chen, H.L. and Wissawapaisal, K (2001)."Application of Wigner-Ville Transform to Evaluate Tensile Forces in Seven-Wire Prestressing Strands", Submitted to Journal of Engineering Mechanics. 